\title{
Investigation of a Planetary Differential for Use as a Continuously Variable Transmission
}

Austin B. Randall

Brigham Young University - Provo

Follow this and additional works at: https://scholarsarchive.byu.edu/etd

Part of the Mechanical Engineering Commons

\section{BYU ScholarsArchive Citation}

Randall, Austin B., "Investigation of a Planetary Differential for Use as a Continuously Variable Transmission" (2012). Theses and Dissertations. 3311.

https://scholarsarchive.byu.edu/etd/3311

This Thesis is brought to you for free and open access by BYU ScholarsArchive. It has been accepted for inclusion in Theses and Dissertations by an authorized administrator of BYU ScholarsArchive. For more information, please contact scholarsarchive@byu.edu, ellen_amatangelo@byu.edu. 
Investigation of a Planetary Differential for Use as a

Continuously Variable Transmission

Austin B. Randall

A thesis submitted to the faculty of

Brigham Young University

in partial fulfillment of the requirements for the degree of

Master of Science

Robert H. Todd, Chair

Brian D. Jensen

Larry L. Howell

Department of Mechanical Engineering

Brigham Young University

August 2012

Copyright (C) 2012 Austin B. Randall

All Rights Reserved 


\title{
ABSTRACT \\ Investigation of a Planetary Differential for Use as a Continuously Variable Transmission
}

\author{
Austin B. Randall \\ Department of Mechanical Engineering, BYU \\ Master of Science
}

With gas prices on the rise, the demand for high-mileage and low pollution vehicles has taken on an unprecedented role in our society. The production and implementation of electric and hybrid-electric vehicles has recently been a large focus of all major automobile manufacturers. Although these new vehicles have begun to solve much of the expensive fuel consumption and air pollution problems that our economy faces, the initial cost of these vehicles has proven to still be too expensive to capture a significant portion of the market. The further advancement of this technology must not only continue to focus on better fuel efficient and decreased pollution producing vehicles, but also decrease the cost of these vehicles to make them more available and enticing to the general public.

Results from this research include one potential solution to reduce the cost of electric and hybrid-electric vehicles. Previous research performed in this area has led to the investigation and bench-top testing of a special type of mechanical system known as a Planetary Differential (PD). An exploration of the functionality of this system has shown that the PD can simplify expensive and complex electronic control systems for electric and hybrid-electric vehicles, thus reducing the cost to the consumer. In this study, fundamental speed, torque and power relationships for the PD were developed and tested under various loading conditions. Advantages and disadvantages of the PD, as compared to other similar mechanical systems, are identified and outlined. Recommendations for future work and implementation of the PD in electric and/or hybrid-electric vehicles are presented herein.

Keywords: Austin Randall, hybrids, CVT, HEV, EV, planetary differential, continuously variable transmission, infinitely variable transmission 


\section{ACKNOWLEDGMENTS}

I wish to acknowledge all individuals and institutions that have aided and provided guidance to myself throughout, and leading up to, the process of performing and creating this work.

I am grateful to Brigham Young University and the Ira A. Fulton College of Engineering and Technology for the use of their facilities and many other available resources.

I would like to thank the Department of Mechanical Engineering and many of their faculty members that have been an inspiration and motivation for me to set my goals high and reach beyond my perceived potential. I include a special thanks to Dr. Dale R. Tree who has become a great friend and has always been willing to provide counsel and advice regarding educational matters. I also include Dr. Kenneth W. Chase, Ken Forster and Kevin Cole who have provided insight and support in times when it was needed.

I acknowledge a team of faculty and students at Brigham Young University in Rexburg, Idaho who aided in the design, and built, the system tested in this research. I recognize contributions from Dr. Alan Dutson, Aaron Schellenberg (Team Coach) and members of the respective Capstone Team: Ben Ward, Brett Hale, Leighton Roberts, Phillip Field and Dan Price.

I appreciate and acknowledge the help of fellow graduate students and other fellow researchers for whom I have developed a respect and lasting friendship. I specifically include the help of Mike Sanders, Cody Telford and John Wyall.

I would also like to express my appreciation to my graduate committee members including: Dr. Robert H. Todd (Advisor), Dr. Brian D. Jensen and Dr. Larry L. Howell. Each of these professors have not only provided feedback on my graduate work, but have been an example in word and deed. I especially appreciate the help and wisdom of Dr. Todd who has been far more than just an advisor on academic matters, but has believed in my efforts and continued to push and motivate me toward excellence in all areas of my life. 
I am grateful to my wonderful parents, Bryce and Karin Randall, who laid the foundation of sacrifice and hard work for me to acomplish great things in life and take advantage of all that has been provided for me due to the sacrifices of many men and women that have gone before me.

Above all other mortal help and support, I acknowledge and thank my beautiful wife Amy and daughters Bella, Abby and Hayley (and one more on the way) that have sacrificed countless hours in support of my educational goals and dreams.

Lastly, no acknowledgement would be complete without giving recognition to The Great Creator of heaven and earth who has given and provided mankind with all that he has. I recognize the wisdom and love of my Heavenly Father who has blessed me with priceless opportunities and guidance throughout my life including all education opportunities that have been mine to enjoy. 


\section{TABLE OF CONTENTS}

LIST OF TABLES $\ldots \ldots \ldots \ldots \ldots \ldots \ldots \ldots \ldots \ldots$ vii

LIST OF FIGURES $\ldots \ldots \ldots \ldots \ldots \ldots \ldots$ viii

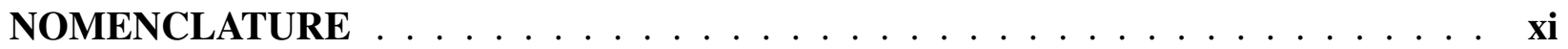

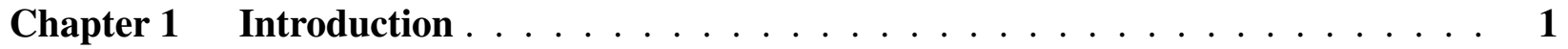

1.1 Rising Gas Prices . . . . . . . . . . . . . . . . . . . . 1

1.2 Cost of Hybrid Vehicles . . . . . . . . . . . . . . . . . . . . . 2

1.3 Electronic Controllers . . . . . . . . . . . . . . . . . . . . . . . 3

1.4 Research Objectives . . . . . . . . . . . . . . . . . . . . . . . . 4

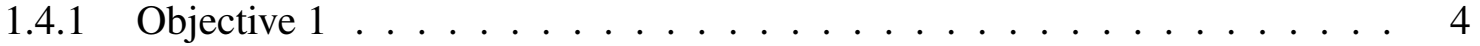

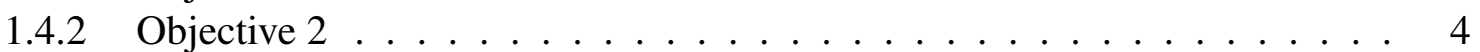

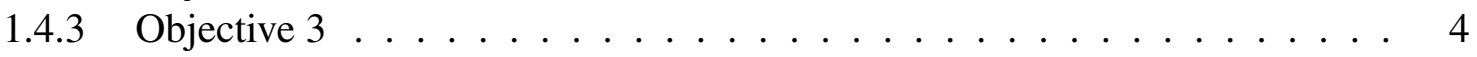

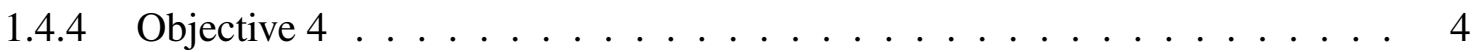

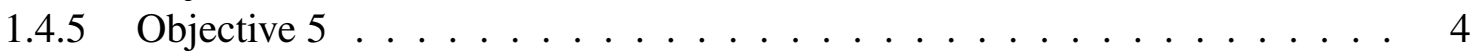

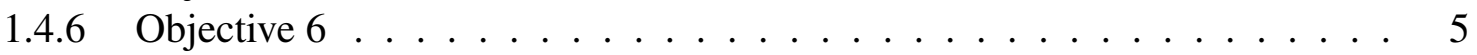

1.5 Proposed Research . . . . . . . . . . . . . . . . . . . . . . 5

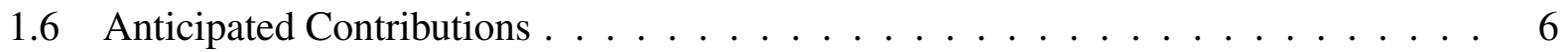

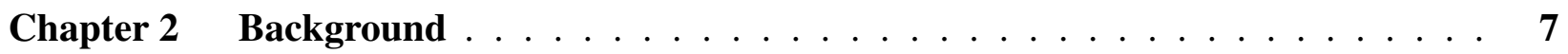

2.1 History of the Automotive Transmission . . . . . . . . . . . . . . . 7

2.2 Recent Innovation . . . . . . . . . . . . . . . . . . . . . . . . . 8

2.3 Purpose of a Transmission $\ldots \ldots \ldots \ldots \ldots$

2.4 Types of Automotive Transmissions _ . . . . . . . . . . . . . . . . 10

2.4.1 Manual Transmissions . . . . . . . . . . . . . . . . . . . 10

2.4.2 Automatic Transmissions . . . . . . . . . . . . . . . . . . . . . 10

2.4.3 Dual-Clutch Transmissions . . . . . . . . . . . . . . . . . . . . 11

2.4.4 Continuously Variable Transmissions _. . . . . . . . . . . . . . . . . . 11

2.4.5 Electric Vehicle Transmissions . . . . . . . . . . . . . . . . . . . . 14

2.5 Types of 3 Input/Output Systems . . . . . . . . . . . . . . . . . . . . . . 16

2.5.1 Standard Automotive Differentials . . . . . . . . . . . . . . . . . . . 17

2.5.2 Gleason-Torsen Differentials . . . . . . . . . . . . . . . . . . . . . 18

2.5 .3 Planetary Gear Sets . . . . . . . . . . . . . . . . . . . . 20

2.5.4 Variable-Geometry CVT . . . . . . . . . . . . . . . 21

2.5 .5 Hydrostatic CVT . . . . . . . . . . . . . . . . . . . 22

2.5 .6 Electric CVT . . . . . . . . . . . . . . . . . . . 23

2.5.7 Planetary Differentials . . . . . . . . . . . . . . . . . . . . 24

2.6 Previous Research . . . . . . . . . . . . . . . . . . . . . . . . . . . 26

2.6 .1 Tests Performed $\ldots \ldots \ldots \ldots \ldots \ldots$

2.6 .2 Efficiency . . . . . . . . . . . . . . . . . . 33

2.6 .3 Problems Identified . . . . . . . . . . . . . . . . . . 35 
Chapter 3 Planetary Differential as a Potential Solution _ . . . . . . . . . . . . . 39

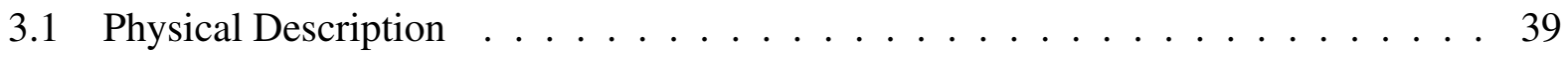

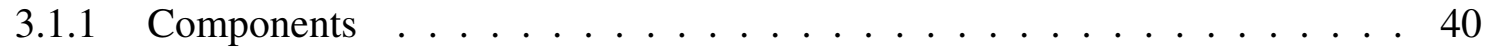

3.1 .2 Dimensions . . . . . . . . . . . . . . . . . . . . 42

3.1 .3 Gears . . . . . . . . . . . . . . . . . . . . . 42

3.1 .4 Material Properties . . . . . . . . . . . . . . . . . . . . . . 42

3.2 Governing Equations . . . . . . . . . . . . . . . . . . . . . . . 43

3.3 Experimental Setup . . . . . . . . . . . . . . . . . . . . . . . 47

3.3 .1 Physical Layout . . . . . . . . . . . . . . . . . . . . . . . . . 47

3.3.2 Electric Motors . . . . . . . . . . . . . . . . . . . . . . . 48

3.3 .3 Data Collection . . . . . . . . . . . . . . . . . . 52

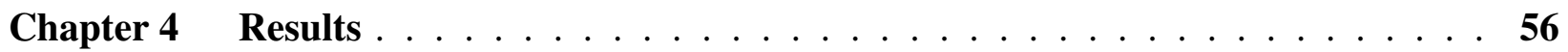

4.1 Repeat of Previous Testing . . . . . . . . . . . . . . . 56

$4.1 .1 \quad$ RPM Measurements . . . . . . . . . . . . . . . . . . . . 56

4.1 .2 Torque Measurements . . . . . . . . . . . . . . . . . . 62

4.1 .3 Power Measurements . . . . . . . . . . . . . . . . . . . . . . . 67

4.1 .4 Efficiency Measurements . . . . . . . . . . . . . . . . . . . 72

4.1 .5 Implications . . . . . . . . . . . . . . . . . 77

4.2 Additional Testing . . . . . . . . . . . . . . . . . . . 77

4.2.1 RPM Measurements . . . . . . . . . . . . . . . . . . . . . . 78

4.2 .2 Torque Measurements . . . . . . . . . . . . . . . . . . . . . . . . . 79

4.2 .3 Power Measurements . . . . . . . . . . . . . . . . . . . . . . . 86

4.2 .4 Efficiency Measurements . . . . . . . . . . . . . . . . . . . 86

4.2 .5 Implications . . . . . . . . . . . . . . . . . . . . . . 93

4.3 Efficiency vs. Output Speed . . . . . . . . . . . . . . . . . . . 93

4.4 Back-Drive Problem . . . . . . . . . . . . . . . . . . . . . . . . . . . 99

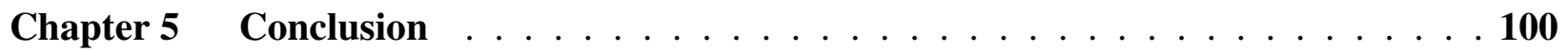

5.1 How Research Objectives were Accomplished . . . . . . . . . . . . . . . . . . 100

5.1 .1 Objective $1 \ldots \ldots \ldots \ldots$

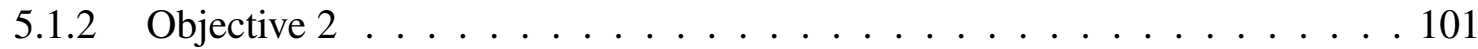

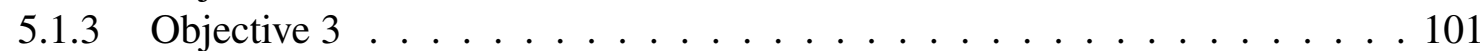

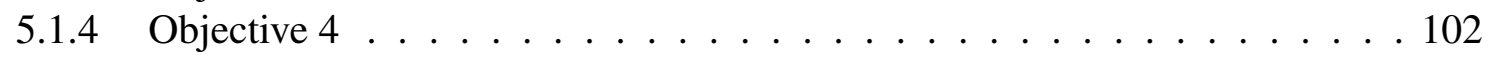

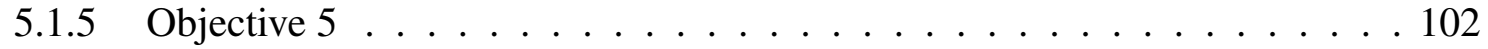

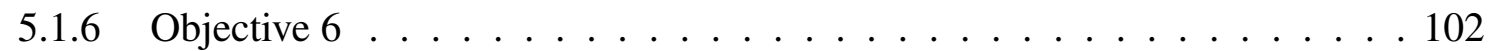

5.2 A Final Word . . . . . . . . . . . . . . . . . . . . . . . . . . 104

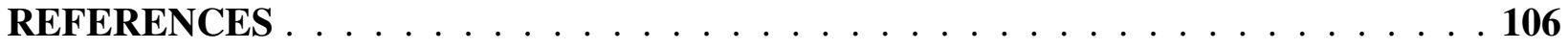

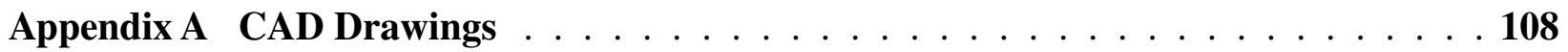




\section{LIST OF TABLES}

2.1 The Physical Effects of Electric Currents . . . . . . . . . . . . . . . . . . 16

2.2 Comparison of Various $3 \mathrm{I} / \mathrm{O}$ Systems $\ldots \ldots \ldots \ldots$

2.3 Efficiency of the Gleason-Torsen Differential . . . . . . . . . . . . . . 38

3.1 PD Basic Dimensions . . . . . . . . . . . . . . . . . . . . . . . . . . . . . . 42

3.2 PD Part Material Properties . . . . . . . . . . . . . . . . 43

3.3 Gear Properties of the PD . . . . . . . . . . . . . . . . . 45

3.4 Gear Ratio Properties of the PD . . . . . . . . . . . . . . . . . 45

3.5 Example of using the Tabular Method . . . . . . . . . . . . . . . . . . . . . . . . . . .

3.6 Another Example of using the Tabular Method . . . . . . . . . . . . . . . 46

3.7 Specifications of DC Motors . . . . . . . . . . . . . . . . 50

3.8 Specifications of Encoders . . . . . . . . . . . . . . . 51

3.9 Torque Sensor Locational Information . . . . . . . . . . . . . . . . . . . . . . . . . . . . . . . 52

3.10 Additional Torque Sensor Information . . . . . . . . . . . . . 53

4.1 Highest Achieved Efficiency Comparison . . . . . . . . . . . . . . . 77

4.2 Numerical Values for RPM and Load Variations . . . . . . . . . . . . . . . . . . 78

4.3 Efficiency Comparison of all 9 Tests . . . . . . . . . . . . . . . 93 


\section{LIST OF FIGURES}

1.1 Average U.S. Gasoline Prices 1992 - 2012 . . . . . . . . . . . . . . . . . . 1

1.2 Electronic Controller for the Toyota Prius . . . . . . . . . . . . . . . 2

1.3 Standard Electric Vehicle Architecture . . . . . . . . . . . . . . . . . 3

1.4 Simplified Version of a $3 \mathrm{I} / \mathrm{O}$ System $\ldots \ldots \ldots \ldots$

2.1 Friction-Based Continuously Variable Transmission _ . . . . . . . . . . . . 12

2.2 Push-Belt Type Friction-Based CVT . . . . . . . . . . . . . . . . . . . . . . 13

2.3 Traction-Based Toroidal CVT Functionality . . . . . . . . . . . . . . . . 14

2.4 Powertrain Layout of the Toyota Prius . . . . . . . . . . . . . . . . 15

2.5 Modified Powertrain Layout of the Toyota Prius . . . . . . . . . . . . . . . . 18

2.6 Standard Automotive Differential . . . . . . . . . . . . . . . . . . . . . . . . . . . . 19

2.7 Gleason-Torsen Differential . . . . . . . . . . . . . . . . . . . . . . . . . . . . . 19

2.8 Typical Planetary Gear Set . . . . . . . . . . . . . . . . . . . . . . 21

2.9 Variable-Geometry or Pivot Arm CVT . . . . . . . . . . . . . . . . . 22

2.10 Exposed View of a Hydrostatic CVT . . . . . . . . . . . . . . . . . . . . 23

2.11 Simplified Version of an Electric CVT . . . . . . . . . . . . . . . . 24

2.12 Planetary Differential . . . . . . . . . . . . . . . . . . . . . . . 25

2.13 Test $1 \mathrm{~s}-$ Angular Velocity . . . . . . . . . . . . . . . . . . 28

2.14 Test $1 \mathrm{~s}-$ Torque . . . . . . . . . . . . . . . . . . . . . . . . . . . . . 29

2.15 Test $1 \mathrm{~s}$ - Power . . . . . . . . . . . . . . . . . . . . . . . . . . . . 29

2.16 Test $1 \mathrm{~s}$ - Efficiency . . . . . . . . . . . . . . . . . . . 30

2.17 Test $2 \mathrm{~s}$ - Angular Velocity . . . . . . . . . . . . . . . . . 30

2.18 Test $2 \mathrm{~s}-$ Torque . . . . . . . . . . . . . . . . . . . . 31

2.19 Test $2 \mathrm{~s}$ - Power . . . . . . . . . . . . . . . . . . . . . . 31

2.20 Test 2 s - Efficiency . . . . . . . . . . . . . . . . . . . . . . 32

2.21 Test $1 \mathrm{o}-$ Angular Velocity $\ldots \ldots \ldots 32$

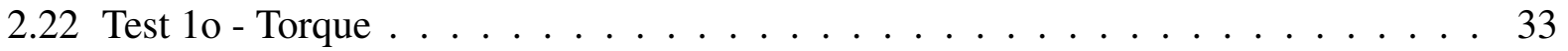

2.23 Test 1 o- Power . . . . . . . . . . . . . . . . . . . . 33

2.24 Test 1 o- Efficiency . . . . . . . . . . . . . . . . . . . . . . . 34

2.25 Test 20 - Angular Velocity . . . . . . . . . . . . . . . . . . 34

2.26 Test 20 - Torque . . . . . . . . . . . . . . . . . . . . 35

2.27 Test 20 - Power . . . . . . . . . . . . . . . . . . . . . . . 35

2.28 Test 20 - Efficiency . . . . . . . . . . . . . . . . . . . 36

2.29 Test Crossover - Angular Velocity . . . . . . . . . . . . . . . . . . 36

2.30 Test Crossover - Torque . . . . . . . . . . . . . . . . . . . . . . . 37

2.31 Test Crossover - Power . . . . . . . . . . . . . . . . . . . . . . . . . . . 37

2.32 Test Crossover - Efficiency . . . . . . . . . . . . . . . . . . . 38

3.1 Planetary Differential . . . . . . . . . . . . . . . . . . . . . . . . 39

3.2 Cutaway Views of the PD . . . . . . . . . . . . . . . . . . 41

3.3 Planetary Differential with labeled I/Os . . . . . . . . . . . . . . . . . 44

3.4 Experimental Setup for Testing of the PD . . . . . . . . . . . . . . . . 48

3.5 Simplified view of Experimental Setup . . . . . . . . . . . . . . . . . . 49 
3.6 AC Motor used as Prime Mover . . . . . . . . . . . . . . . . . . . . . . . . . . 49

3.7 DC Motor used as Control Motor . . . . . . . . . . . . . . . . . . . . . . . 50

3.8 DC Motor used as Output Motor . . . . . . . . . . . . . . . . . . 51

3.9 Mechanical Braking Device used to Simulate Loading . . . . . . . . . . . . . . . 52

3.10 Torque Sensors used at each of the $3 \mathrm{I} / \mathrm{Os} \ldots \ldots$. . . . . . . . . . . . 53

3.11 Data Aquisition . . . . . . . . . . . . . . . . . . 54

3.12 Labview Program used for Testing the PD . . . . . . . . . . . . . . 55

4.1 Test $1 \mathrm{~s}$ - Comparison of Anglular Velocity . . . . . . . . . . . . . . . 57

4.2 Test $2 \mathrm{~s}$ - Comparison of Anglular Velocity . . . . . . . . . . . . . . . . 58

4.3 Test 1 o - Comparison of Anglular Velocity . . . . . . . . . . . . . . . . . . 59

4.4 Test $2 \mathrm{o}$ - Comparison of Anglular Velocity . . . . . . . . . . . . . . . . . 60

4.5 Test Crossover - Comparison of Anglular Velocity . . . . . . . . . . . . . . . 61

4.6 Test $1 \mathrm{~s}$ - Comparison of Torque . . . . . . . . . . . . . . . . . . 62

4.7 Test $2 \mathrm{~s}$ - Comparison of Torque . . . . . . . . . . . . . . . . . 63

4.8 Test $1 \mathrm{o}$ - Comparison of Torque . . . . . . . . . . . . . . . . 64

4.9 Test $2 \mathrm{o}$ - Comparison of Torque . . . . . . . . . . . . . . . . . . . 65

4.10 Test Crossover - Comparison of Torque . . . . . . . . . . . . . . . 66

4.11 Test $1 \mathrm{~s}$ - Comparison of Power . . . . . . . . . . . . . . . . . . . . 67

4.12 Test $2 \mathrm{~s}$ - Comparison of Power . . . . . . . . . . . . . . . . . . 68

4.13 Test 1 o - Comparison of Power . . . . . . . . . . . . . . . . . . 69

4.14 Test 2o-Comparison of Power . . . . . . . . . . . . . . . . . 70

4.15 Test Crossover - Comparison of Power . . . . . . . . . . . . . . . . . 71

4.16 Test $1 \mathrm{~s}$ - Comparison of Efficiency . . . . . . . . . . . . . . . . 72

4.17 Test 2s - Comparison of Efficiency . . . . . . . . . . . . . . . . . 73

4.18 Test 1 o - Comparison of Efficiency . . . . . . . . . . . . . . . . . . 74

4.19 Test 2o - Comparison of Efficiency . . . . . . . . . . . . . . . . 75

4.20 Test Crossover - Comparison of Efficiency . . . . . . . . . . . . . . 76

4.21 RPM Measurements at High RPM with Varying Loads . . . . . . . . . . . . . 80

4.22 RPM Measurements at Medium RPM with Varying Loads . . . . . . . . . . . . 81

4.23 RPM Measurements at Low RPM with Varying Loads . . . . . . . . . . . . . 82

4.24 Torque Measurements at High RPM with Varying Loads . . . . . . . . . . . . 83

4.25 Torque Measurements at Medium RPM with Varying Loads . . . . . . . . . . . . 84

4.26 Torque Measurements at Low RPM with Varying Loads . . . . . . . . . . . . . . 85

4.27 Power Measurements at High RPM with Varying Loads . . . . . . . . . . . . . . 87

4.28 Power Measurements at Medium RPM with Varying Loads . . . . . . . . . . . . 88

4.29 Power Measurements at Low RPM with Varying Loads . . . . . . . . . . . . . 89

4.30 Efficiency Measurements at High RPM with Varying Loads . . . . . . . . . . . . . 90

4.31 Efficiency Measurements at Medium RPM with Varying Loads . . . . . . . . . . . 91

4.32 Efficiency Measurements at Low RPM with Varying Loads . . . . . . . . . . . . 92

4.33 Efficiency vs. Speed at High RPM with Varying Loads . . . . . . . . . . . . . . 95

4.34 Efficiency vs. Speed at Medium RPM with Varying Loads . . . . . . . . . . . . 96

4.35 Efficiency vs. Speed at Low RPM with Varying Loads . . . . . . . . . . . . . 97

4.36 Efficiency vs. Speed of 4 Automobile Transmissions . . . . . . . . . . . . . 98 
5.1 Experimental Setup with Back-drive Solution ․ . . . . . . . . . . . . . 103

5.2 Electric-Only Powertrain Layout . . . . . . . . . . . . . . . . . . . . . 105

5.3 Hybrid-Electric Powertrain Layout . . . . . . . . . . . . . . . . . 105 


\section{NOMENCLATURE}

$\begin{array}{ll}P & \text { Power } \\ T & \text { Torque } \\ A & \text { Amperes } \\ r p m & \text { Revolutions per minute } \\ \omega_{\text {out }} & \text { Speed of the Output } \\ \omega_{\text {in } 1} & \text { Speed of the First Input } \\ \omega_{\text {in } 2} & \text { Speed of the Second Input } \\ r p m A & \text { Speed of Input A measured in revolutions per minute } \\ r p m B & \text { Speed of Input B measured in revolutions per minute } \\ r p m C & \text { Speed of Output C measured in revolutions per minute } \\ T_{A} & \text { Torque seen at Input A } \\ T_{B} & \text { Torque seen at Input B } \\ T_{C} & \text { Torque seen at Output C } \\ \eta_{\text {mech }} & \text { Mechanical efficiency } \\ P_{\text {out }} & \text { Power out of the system } \\ P_{\text {in }} & \text { Power into the system } \\ P_{\text {loss }} & \text { Power lost in the system } \\ h p & \text { Horsepower } \\ N & \text { Revolutions per minute }\end{array}$




\section{CHAPTER 1. INTRODUCTION}

\subsection{Rising Gas Prices}

Improving the efficiency and reducing the pollution of modern automobiles has created the opportunity for developing a variety of new technologies. Among these new technologies are the latest in automotive transmissions. The main function of an automotive transmission is to convert power from the engine or electric motor to motion of the vehicle with as little energy loss as possible. With the constant rise in oil prices, these losses have become even more critical to the consumer, and therefore, the manufacturer. The demand for the fuel efficient and low emission vehicle has never been more important. As seen in Figure 1.1, gas prices have been on the rise for the last decade and are expected to continue to rise [1].

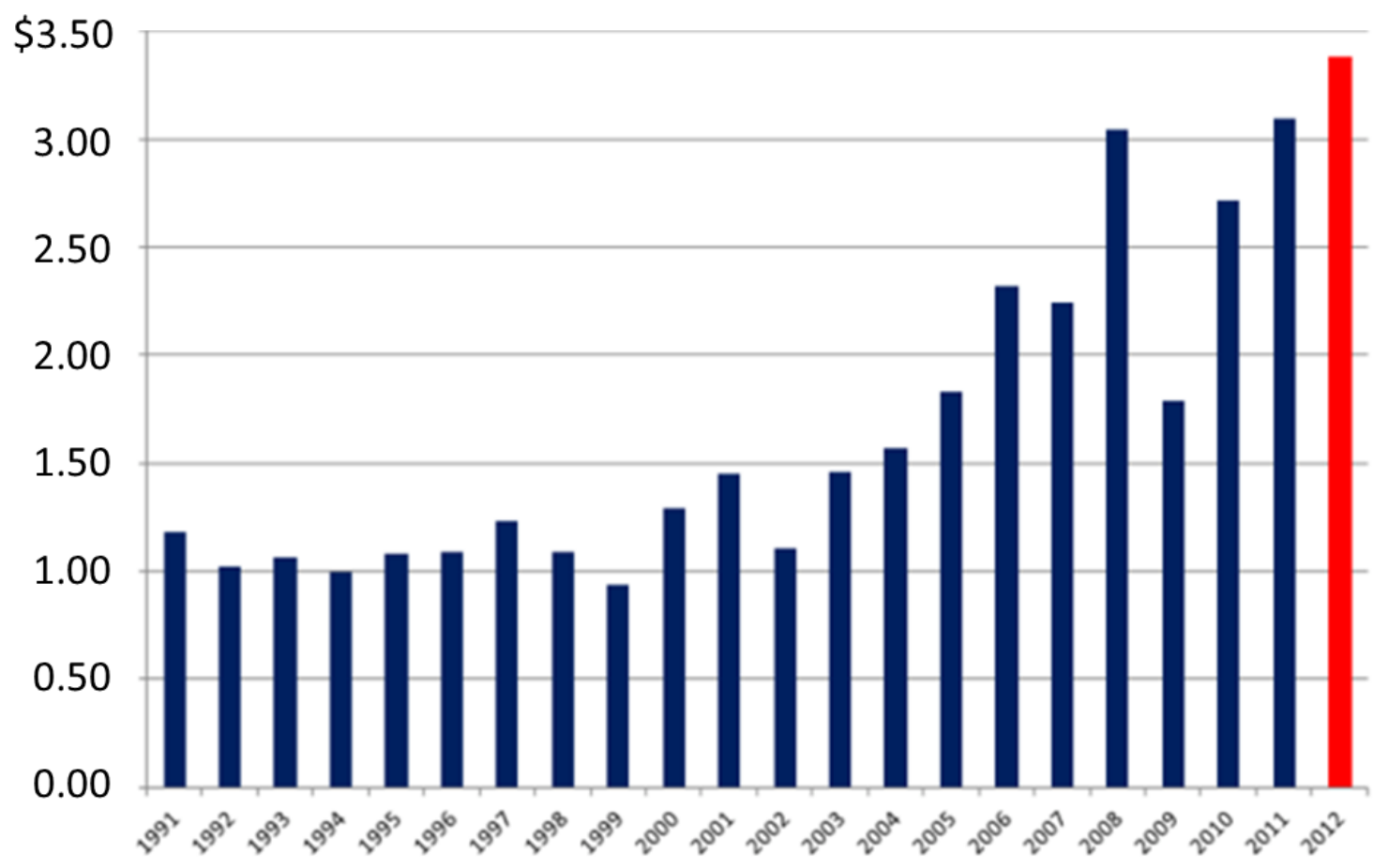

Figure 1.1: Average U.S. Gasoline Prices 1992 - 2012 
Due to the increase in gas prices, the research, development and production of highly fuel efficient vehicles, including hybrids, has taken on an unprecedented role in our economy. Currently in our market there are several manufacturers that produce a variety of types of electric or hybrid-electric high-mileage vehicles, all of which can be classified into the following three categories: Electric-only Vehicles, Hybrid-Electric Vehicles (HEV) and Plug-in Hybrid Electric Vehicles (PHEV). For the purpose of this research all types shall hereafter be referred to as Electrified Vehicles (EVs).

\subsection{Cost of Hybrid Vehicles}

Although there are numerous EVs available to the consumer, they have proven to still be too expensive to gain a significant share of the market. The two main contributors to their cost are high-powered battery packs and the electronic controls used to manage the power from the battery packs. The main electronic system used to manage this power is typically referred to as the electronic controller (See Figure 1.2).

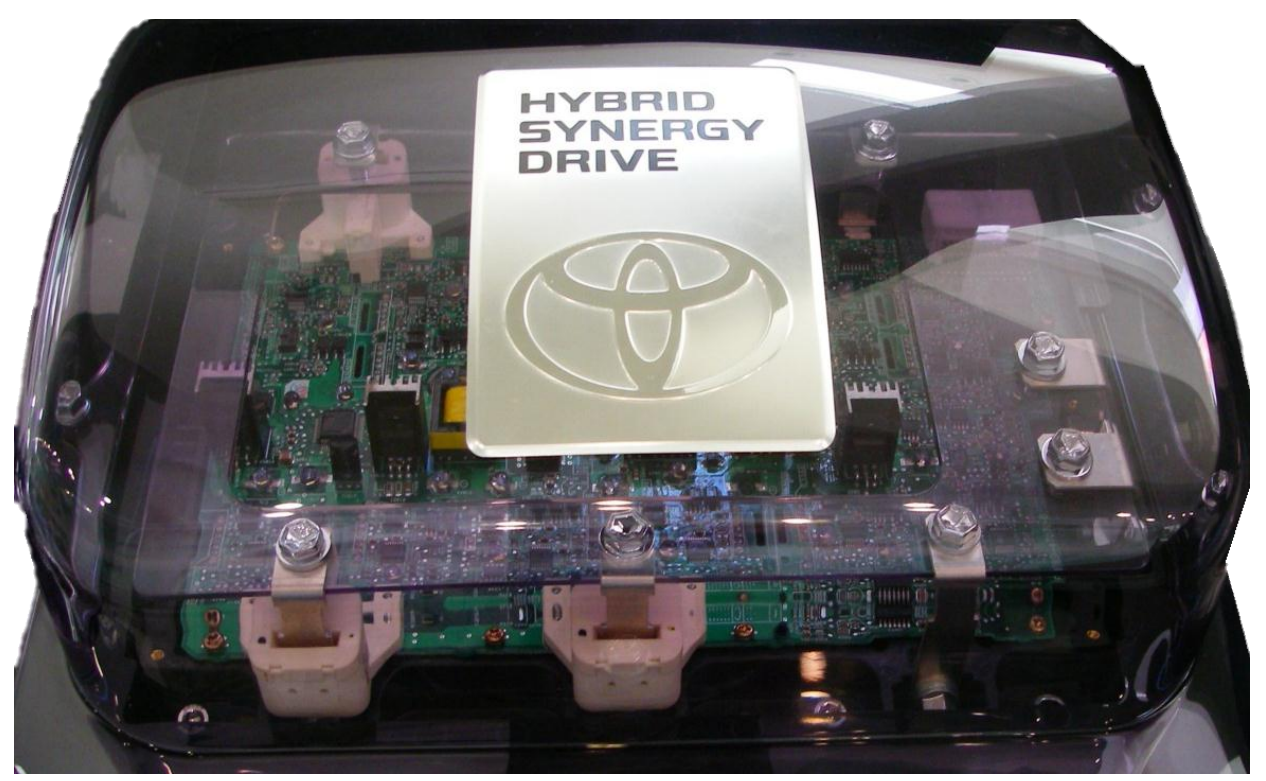

Figure 1.2: Electronic Controller for the Toyota Prius 


\subsection{Electronic Controllers}

Electronic controllers are used to govern and manage electric power released from the batteries and appropriately direct it to the electric motor(s). Nearly all EVs follow the same basic setup as seen in Figure 1.3. The battery pack supplies a desired amount of power based on its voltage and current. Power can be calculated from voltage and current by using the following equation:

$$
P=V * A
$$

where $P$ is power measured in Watts, $V$ is voltage measured in Volts, and $A$ is current measured in Amperes [2].

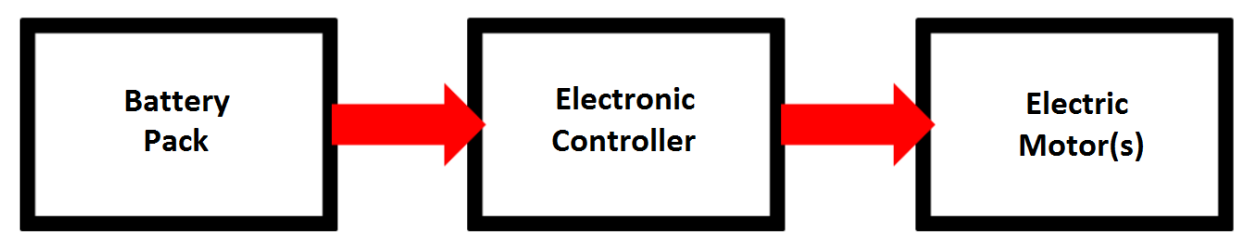

Figure 1.3: Standard Electric Vehicle Architecture

Because all electric power causing motion of the vehicle must travel through the controller, the size and complexity of its internal components must be capable of managing high power. If the controller didn't need to manage all this power, or if there was another way to manage the power, the cost of the controller could be reduced, thus reducing the overall cost of EVs. It would, therefore, be beneficial to investigate alternative methods to see if a solution can be found. One potential alternative solution would be to use a mechanical system to replace a significant portion of the functionality of the electronic controller.

One such mechanical system has already been tested in previous research by two former graduate students, Dax Wells and Benjamin Groen [3] [4]. Wells and Groen tested a GleasonTorsen Differential (GTD), which was coupled with two inputs that contributed to a single output. By varying one of the two inputs, the state of the ratio between the other input and output of the system also varied. Wells and Groen both determined that, although the system they tested 
demonstrated its functionality, a more efficient and/or appropriate system would be required for application in modern EVs.

\subsection{Research Objectives}

The main objective of this research is to more fully define the characteristics of a dual-input, single-output system that will achieve a higher efficiency than the previously tested Gleason-Torsen Differential [3] [4]. The following are additional objectives that will aid in accomplishing the main objective.

\subsubsection{Objective 1}

Obtain a working knowledge (mathematical and physical) of various types of 3 Input/Output (I/O) systems, including their efficiencies.

\subsubsection{Objective 2}

Select and build (or have built) the appropriate type of $3 \mathrm{I} / \mathrm{O}$ system.

\subsubsection{Objective 3}

Test this system under various power combinations (i.e. vary the torque and speed of the different I/Os).

\subsubsection{Objective 4}

Record the pertinent data during testing.

\subsubsection{Objective 5}

Analyze and compare the recorded data, including comparing it to the research performed previously. 


\subsubsection{Objective 6}

Make recommendations for future research and innovation based on these findings.

\subsection{Proposed Research}

The selected system (a simplified version is seen in Figure 1.4) will mainly be tested under the following conditions, where "Input A and B" represent the two inputs to the system and "Output C" represents the single output: Input A will be held at a constant rpm, Input B will vary in speed, and Output $\mathrm{C}$ will provide the combined output result of the two inputs [3]. The torque and/or rpm of the two inputs will both vary to provide varying torques and speed for the output.

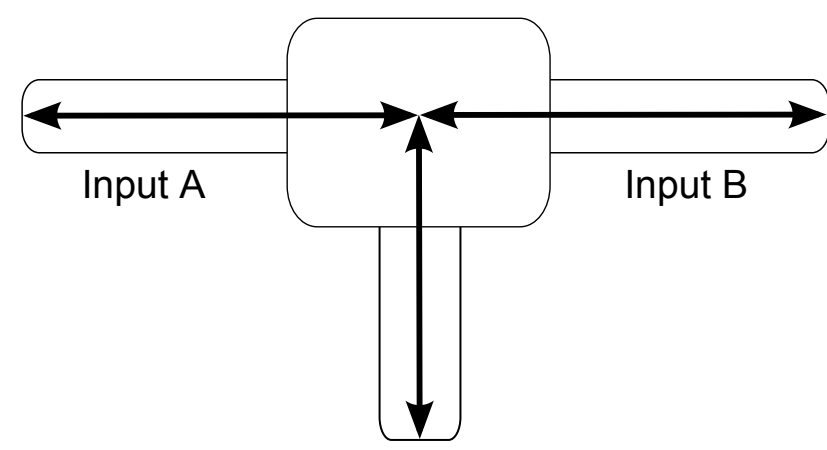

Output C

Figure 1.4: Simplified Version of a 3 I/O System

To fully investigate the selected system, the following data will be collected and calculated during and after the proposed testing:

- speed

- torque

- power

- efficiency 


\subsection{Anticipated Contributions}

It is anticipated that this research will aid in identifying the parameters and their characteristics for this type of mechanical system with application for use as a Continuously Variable Transmission (CVT). This research will aid in the design of future automotive transmissions that allow, not only a step-less ratio change to maintain higher efficiency, but also constant-mesh gearing that will allow larger-powered vehicles to obtain higher efficiency as well. Above and beyond these reasons, it is expected that this research will also aid in reducing the cost of EVs, making them more affordable in today's market.

Another particular application of this research, besides reducing EV cost, could be in hybrid vehicle drivetrains that can take advantage of multiple-input and single-output transmissions. A gas/electric hybrid vehicle could have a gasoline or diesel engine running at a constant or near constant rpm yet varying highway speeds of the vehicle. This would allow the vehicle decreased fuel consumption and higher efficiency overall due to the system remaining constantly meshed through its gears, instead of being frictionally-based like current automotive and other vehicle CVTs.

It is also anticipated that the results of this research will be published in an accredited automotive engineering journal such as the International Journal of Powertrains or one of the two SAE International Journals of Passenger Cars. This research also has potential for presentation in the ASME Design Engineering Technical Conference. 


\section{CHAPTER 2. BACKGROUND}

\subsection{History of the Automotive Transmission}

Although the modern automotive transmission is relatively efficient, it has taken over a century to develop many of the improvements that are available today. The earliest automobile transmission was designed by Louis-Rene Panhard and Emile Levassor of France in the year 1894 [5]. One year later, Panhard and Levassor presented a fully functioning vehicle with a "3-speed sliding gear transmission" [5]. Along with a few other innovations, including the functioning rear differential, the design of this basic transmission was soon adopted by the majority of the automobile industry and modifications and improvements to the design were well underway.

One problem of the sliding gear transmission was the difficulty involved in the actual changing or shifting of gears. This problem, like most problems, left room for future developments. In 1908, Henry Ford introduced the Model T, and within five years began mass production [6]. Among other improvements to previous vehicles, the Model T included a two-speed planetary-geared transmission coupled with a 20 horsepower engine that, together, were capable of reaching speeds of up to $45 \mathrm{mph}$ [6]. This new planetary-style transmission allowed for much easier shifting of the two gears. Over the years, various other versions of these preliminary types of transmissions have evolved and their efficiencies have been improved. Such innovation has also led to the development of a few types of transmissions that are in a class of their own. In the last decade, friction-based Continuously Variable Transmissions, or CVTs, have been gaining popularity and are now used in a wide variety of low-weight and lower-torque vehicles.

Most of this recent innovation in automotive transmissions has come through small improvements to pre-existing systems. 


\subsection{Recent Innovation}

Due to the increase in demand of high-mileage and low-emission vehicles in the last two decades, new configurations of powertrain designs have been steadily evolving. The following are just some of the advancements made in the last several years.

In 1991, Fitz and Pires from Epilogics, Inc. recognized the need for a special type of transmission they termed "A Geared Infinitely Variable Transmission", which was one of the first CVTs that did not rely on friction as a mode of power transfer [7]. This system, although different than the one tested in this work, demonstrated that the need for a geared mechanical device that could function like a CVT had been recognized many years ago.

Later that decade in 1999, Kluger and Long from the Southwest Research Institute did a study of automatic, manual and CVT transmission efficiencies and predicted that their efficiencies were not capable of any more significant improvement. This study showed that different types of transmissions or powertrain configurations would need to be developed to aid in improving fuel economy and reducing emissions for vehicles, rather than just improving the systems that currently existed [8].

A few years later in 2005, Ai and Anderson from the Timken Company and Ricardo, Inc. introduced a special type of hybrid transmission that achieved better fuel economy, while using less power, than some of the leading hybrid and other non-hybrid vehicle transmissions [9]. One year later, Carl et al. of Purdue University examined the use of another special type of CVT known as a "Power Split" CVT. This type of transmission was capable of providing a continuously varying gear ratio, but with efficiencies more comparable to those of a mechanically-based (or geared) transmission [10].

Just recently in October of 2011, Hosokawa et al. of Schaeffler do Brasil Ltda. (a mechan-

ical systems manufacturer in Brazil) recommended replacing standard differentials with a special type of "lightweight" differential [11]. Hosokawa et al. explained this system's potential of handling the same torque and/or power of other differentials, while using smaller gears, less space and therefore, less weight [11]. The differential described by Hosokawa et al. is a planetary differential much like the one investigated in this research, but designed for a different purpose.

Finally, previous to the research done in this work, Wells and Groen tested a GleasonTorsen Differential (GTD) as a dual-input, single-output device [3] [4]. This was done to demon- 
strate that the GTD, or a system like it, could function as a CVT and potentially replace a portion of the functionality of expensive electronic controllers, thus reducing the cost and pollution of EVs.

Each of the advancements explained has aided in the development of specialized types of automotive transmissions with the potential to increase fuel economy and reduce vehicle emissions, thus allowing electric and hybrid-electric vehicles to be purchased at a lower price.

\subsection{Purpose of a Transmission}

As was stated, the purpose of any transmission is to convert rotary motion from the engine or electric motor to vehicle motion. A transmission takes a torque and speed input, such as from an internal combustion engine and/or electric motor (in a hybrid vehicle), and transfers this torque to the drive wheels of the vehicle. One of the main purposes of the transmission is to allow the input, or power source of the vehicle, to remain at or near a constant rpm, while varying the speed of the vehicle. This allows the fuel, or energy, from the input power source to be used in a more efficient manner. Power is related to torque and speed in the following equation:

$$
P=\frac{T * r p m}{63,025}
$$

where $P$ is power (in units of horsepower), $T$ is torque (in units of in-lb) and rpm is revolutions per minute [12]. It can be seen from this equation that (for constant power) when rpm decreases $T$ must increase; a transmission functions to accomplish this desired result, i.e. to trade speed for torque or visa versa. Through a series of gears (or another medium), torque and speed are interchanged to provide the vehicle with the needed speed and torque for improved vehicle efficiency and performance.

For example, a typical gasoline-powered engine may vary in its operating range from 600 to $6000 \mathrm{rpm}$. It's highest efficiency, however, may be found in a much smaller range of around 2000 to $3000 \mathrm{rpm}$ [13]. To allow a vehicle to take advantage of the efficiency range of the engine, a transmission must change the gear ratio allowing the engine to remain inside this ideal range; while varying the output speed/torque to the wheels. In other words, a transmission allows a vehicle to have a variety of speeds, while maintaining a predetermined engine rpm range and efficiency. Without a transmission a vehicle would be very limited in its range of speed and torque and would, 
therefore, have significantly lower fuel efficiency and performance characteristics. With an appropriate transmission a vehicle can operate at multiple speeds and multiple torques under a much higher fuel efficiency.

\subsection{Types of Automotive Transmissions}

With the many types of automobiles available today there are subsequently various types of transmissions to meet the different needs of each of these specific types of vehicles. This section will give a brief explanation of the various common types of automotive and other related vehicle transmissions.

\subsubsection{Manual Transmissions}

The type of transmission that has been in use the longest in automotive applications is the manual, or standard, transmission. This type of transmission uses multiple sets of singularlyengaged constantly-meshed gears that provide the needed ratios under various driving conditions [14]. When a new gear ratio is needed, the operator moves the shifter and selects the gear set that provides the desired ratio. To disengage one gear set and engage another, a friction-based clutch is depressed via the operator's foot.

Ever since automobiles first came off the production line, the manual transmission has been an inexpensive, yet effective, way to transfer power to the drive wheels of a vehicle. Manual transmissions were first offered in 2 and 3 speed arrangements, but today most range from 5 to 7 speeds (gear sets), depending on the application. Although the manual transmission has been in use for over a century, it is slowly being replaced by other more efficient and/or user-friendly alternatives.

\subsubsection{Automatic Transmissions}

In 1940, General Motors introduced the "Hydra-Matic" transmission, the first automatic transmission to be offered in a production vehicle [15] [16]. This automatic transmission used two

separate inputs, throttle and wheel speed (specifically vacuum pressure from the intake manifold and angular velocity from the transmission output shaft), to determine when to shift to the next 
set of gears and did so without assistance from the operator. Automatic transmissions, although not as efficient as manual transmissions, quickly gained popularity because of the simplicity they provided to the driver [8]. One significant difference in automatic transmissions versus manuals is the introduction of the torque converter, which is a type of fully-automated hydraulic clutch, eliminating the need for the operator to use his/her foot to engage or disengage power from the engine to the transmission.

Automatic transmissions also use a series of Planetary Gear Sets (PGSs), friction-based clutches and bands that allow different gear ratios to be achieved when the inputs dictate doing so. Early automatic transmissions were available in 2 or 3 speeds, but today are offered in arrangements anywhere from 5 to 9 speeds, depending on the application. Although automatic transmissions are still currently very popular, they are also losing some of their long-time market share due to other advancing technologies.

\subsubsection{Dual-Clutch Transmissions}

In the 1980's, Audi and Porsche race cars began using what is known as a Dual-Clutch Transmission (DCT). This type of transmission uses, as the name indicates, two separate frictionbased clutches to engage its gear sets [17]. In the typical setup, one clutch is used to engage the odd numbered gear sets, while the second clutch engages the even numbered sets. The DCT quickly gained popularity for its ability to change gears with minimal loss of acceleration or "down time." Today it is used exclusively in professional racing and is also gaining popularity in modern production vehicles. Although DCTs are now offered in a few select production vehicles, they have introduced their own set of maintenance issues preventing them from capturing a more significant share of the market.

\subsubsection{Continuously Variable Transmissions}

Another type of transmission currently available in automobiles is the Continuously Variable Transmission (CVT). This type of transmission provides a step-less ratio change allowing the power source of the vehicle to maintain high efficiency at all appropriate speeds of the vehicle. Like most transmissions, however, current CVTs have some down sides to their performance. 
CVTs are generally characterized as low torque transmissions [8], due primarily to their reliance on friction as a means of power transfer. For this reason CVTs are not found in vehicles with larger engines, such as full-size trucks, SUVs and higher-powered sports cars.

\section{Friction-Based}

There are several different types of CVTs in use in modern vehicles, each with their own set of advantages and disadvantages. One of the most common types of CVTs used in automobile applications is the Friction-Based CVT. This type of CVT, as seen in Figure 2.1, comes in at least two common varieties, tension-engaged belts and compression-engaged or Push-type belts. Each of these types uses a flexible belt and a set of variable-diameter pulleys that effectively change the input to output gear ratio, while relying on friction between the belt and pulleys to transfer power [18]. The tension-engaged belts function much like those used in common combustion engines to run vehicle accessories like the power steering pump and air conditioning unit. The Push-type belt, as seen in Figure 2.2, uses multiple sets of metal rings contained inside a series of "Thin High-Strength Metal" blocks, which force the belt to maintain its shape under compressive forces.

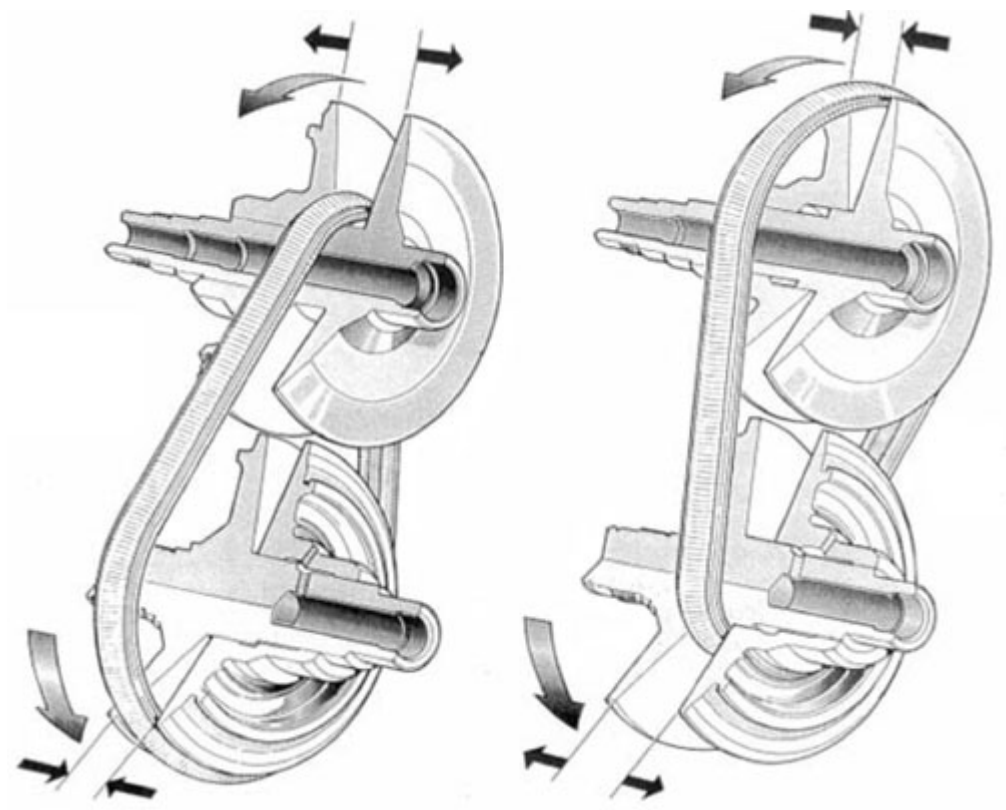

Figure 2.1: Friction-Based Continuously Variable Transmission 


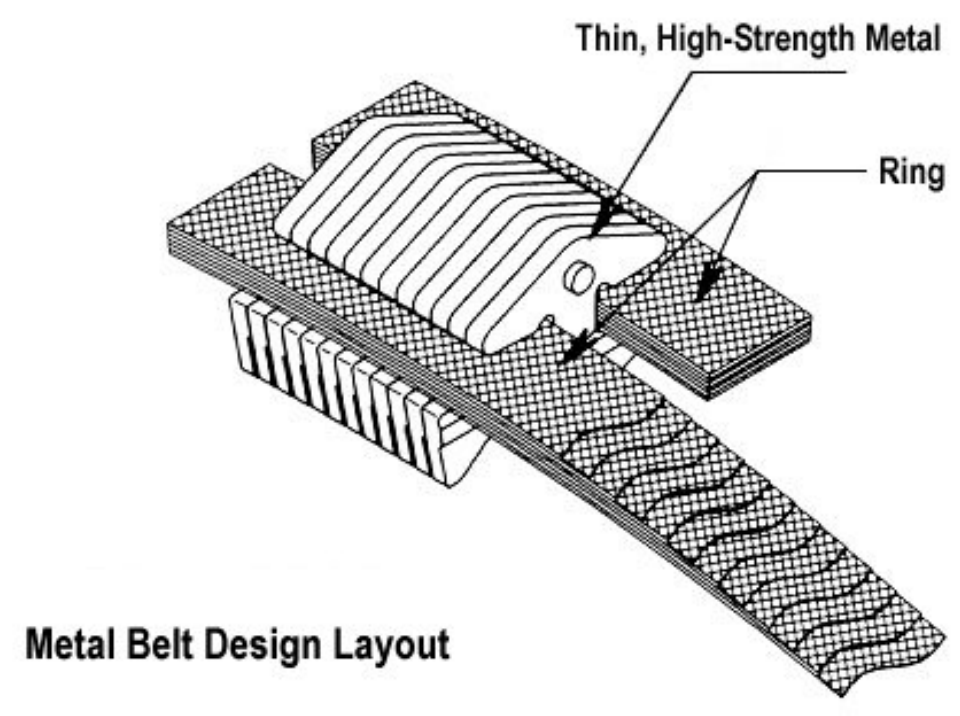

Figure 2.2: Push-Belt Type Friction-Based CVT

As was mentioned earlier, this type of CVT can only be used in low-powered vehicles due to its reliance on friction. Although there are a wide variety of Friction-Based CVTs that are capable of handling enough torque for mid-sized vehicles, none have proven capable of handling the variety of torques provided by all current production vehicles.

\section{Traction-Based}

Another type of CVT in use in modern automobiles is the Traction-Based CVT. This type of CVT, as seen Figure 2.3 (a specific Toroidal Type is shown here), and much like the FrictionBased CVT, also relies on friction as a mode of power transfer, but does so through the use of various wheels and/or discs, as opposed to pulleys and belts. The location of contact (or radial distance) changes, which in turn changes the effective gear ratio of the system. Unfortunately, due to its reliance on friction, this system also promotes significant efficiency loss [19].

As a note to the reader, there are also a few other types of CVTs in use, namely the VariableGeometry, Hydrostatic and Electric CVT, that will be discussed in a later section. These types of CVTs are not used in automobile applications and are, therefore, not included in this section of this work. 


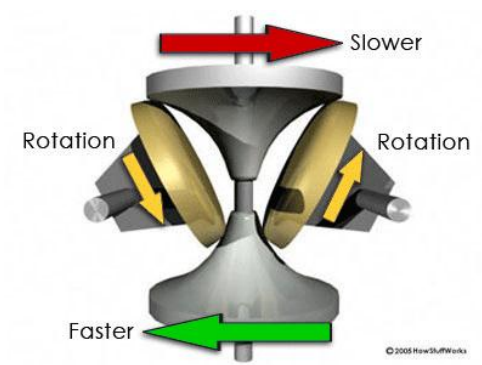

(a) Slow Velocity

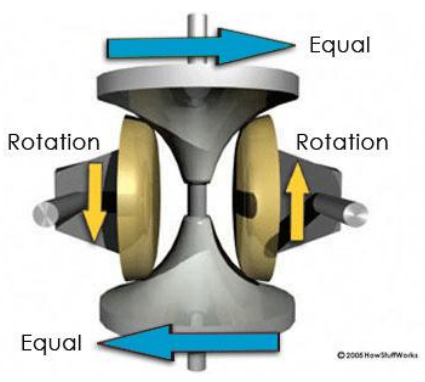

(b) Medium Velocity

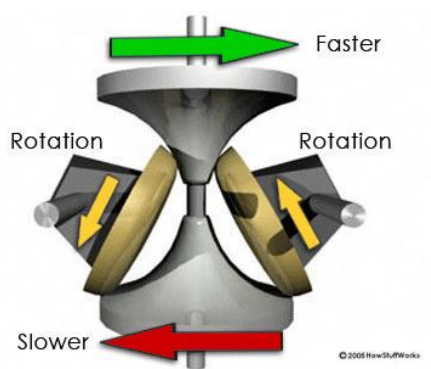

(c) High Velocity

Figure 2.3: Traction-Based Toroidal CVT Functionality

\subsubsection{Electric Vehicle Transmissions}

Hybrid Electric Vehicles (HEVs), which are a type of EV (as noted earlier), are equipped with a special type of transmission that allows them to direct power from multiple input sources to the output of the vehicle [20]. A leader in HEVs is the Toyota Prius. The Prius combines 3 inputs (or power sources) and directs them to the output or drive wheels of the vehicle. Figure 2.4 illustrates the powertrain layout for the Toyota Prius, where the circular gray components represent each of the 3 power sources [21]. Although this only represents one configuration of the many HEVs that are available on the market today, it shows the increased complexity of HEV transmissions as compared to those of conventional internal combustion-powered vehicles that would only receive power from a single source. Due to the increase in complexity of HEV powertrains, current hybrid vehicles retail at a much higher price than their internal combustion competitors, a price that is still too high to achieve a significant share of the market.

As was mentioned earlier, one of the principal cost contributors for all EVs are the expensive electronics including battery and motor control units used to manage and direct power from the battery pack to the vehicle's transmission. These electronics come in many shapes and varieties depending on the vehicle and are given many different names, but shall hereafter be referred to as a single unit known as the "Controller", as labeled in the Figure 2.4.

As high electric power is released from the battery pack it must be appropriately directed to the motor(s) of the vehicle. The motor labeled in Figure 2.4 as the "Traction Motor" is almost always an AC induction motor in today's modern passenger vehicles. Power from batteries for these vehicles, on the other hand, always comes in the form of DC power. The Controller must, 


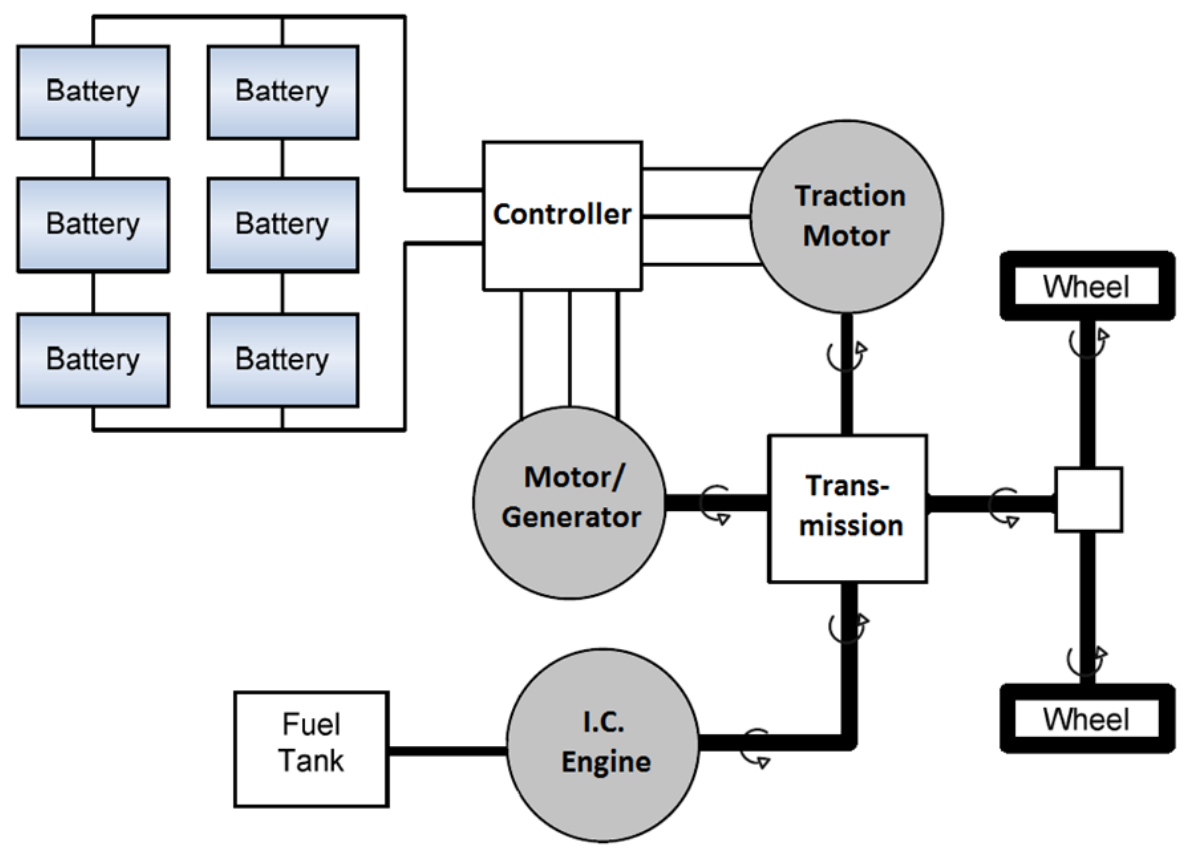

Figure 2.4: Powertrain Layout of the Toyota Prius

therefore, convert DC power from the battery pack to $\mathrm{AC}$ power to allow the vehicle to operate, while regulating the speed of the AC motor. If the Traction Motor for a given EV could simply be turned on or off when needed, instead of constantly regulating its speed through the use of an expensive controller, the cost of the electronics to do so should be less expensive. Also, if this Traction Motor only needed to be turned on or off (i.e. remain at a constant RPM when on) at any given time, then a $\mathrm{DC}$ motor could be used in place of an $\mathrm{AC}$ motor, thus reducing the cost of the vehicle even further as DC power from the batteries would no longer need to be converted to AC power.

Converting from DC to AC power can be a simple task, but doing so for a large amount of power requires more expensive electronic components. A more in-depth look at a few details of the Toyota Prius would be beneficial in explaining the reason for this high cost and some of the motivation behind the main objective of this research.

The high amount of power that must travel through the controller of the Toyota Prius force its design to include components capable of handling high power, and thus increasing its complexity and cost. The Nickel Metal Hydride (NiMH) battery pack for the 2011 Toyota Prius, for 
example, is capable of releasing a peak of 650 Volts and 120 Amps [22]. This amount of power is not only expensive to manage through an electronic controller, but also very dangerous to a technician when the vehicle requires maintenance. When a significant amount of power (by variation of voltage above $30 \mathrm{~V}$ and current above $10 \mathrm{~mA}$ ) penetrates the skin it can have severe effects [23]. Table 2.1, adapted from Electrical Safety Handbook by John Cadick, outlines the serious injury and/or death that can result from contact with current from electrical systems [23].

Table 2.1: The Physical Effects of Electric Currents

\begin{tabular}{|c|c|}
\hline Current $(m A)$ & Physical Sensation \\
\hline$<1$ & Imperceptible \\
1 & Mild Sensation \\
$1-3$ & Painful Sensation \\
10 & Cannot release hand grip \\
30 & Respiratory paralysis (frequently fatal) \\
75 & Heart action discoordinated (probably fatal) \\
250 & Fibrillation threshold 99.5\% \\
4000 & Heart stops (usually fatal) \\
\hline
\end{tabular}

Table 2.1 displays only part of the motivation behind the research explained in this work. A system or solution capable of reducing power seen in electronic controllers could allow for the production of safer EVs. To discover this solution, an investigation of $3 \mathrm{I} / \mathrm{O}$ systems is needed.

\subsection{Types of 3 Input/Output Systems}

A $3 \mathrm{I} / \mathrm{O}$ system (for the purposes of this research) is a mechanical system having two degrees of freedom [24]. This means that if one of the I/Os is held motionless, the second I/O can still rotate and cause the third I/O to rotate at a specific gear ratio. A $3 \mathrm{I} / \mathrm{O}$ system is typically used in one of three arrangements: 1) one I/O acts as the input, while the second I/O is held fixed, allowing the third I/O to rotate at a given ratio, as mentioned above, 2) one of the I/Os is used as an input while the other two I/Os are outputs (i.e. standard use of a typical automotive differential), and 3) two of the I/Os are used as inputs while the remaining $\mathrm{I} / \mathrm{O}$ is used as the output. This third arrangement provides the greatest potential as a possible mechanical solution to expensive 
electronic controllers, which is why a Gleason-Torsen Differential (GTD) was tested in previous research.

The Toyota Prius already uses a system similar to a 3 I/O system, but with an additional I/O. The Prius, however, does not use this system in such a way to eliminate expensive controllers. This research, like the previous research done by Wells and Groen on the GTD, proposes using a $3 \mathrm{I} / \mathrm{O}$ system to replace a portion of the functionality of the electronic controller. This can be done by allowing the bulk of power from the battery pack to be directed to a DC motor as the Traction Motor and only a small amount of power being directed to a second "Motor/Generator", as labeled in Figure 2.4, which would then act as a control motor by further exploiting the potential of a 3 I/O mechanical system. This new proposed arrangement can be seen in Figure 2.5. It can also be seen from Figure 2.5 that although an electronic controller would still be needed to regulate a small control motor, it would not need to be as large, as it would no longer electronically be regulating the Traction Motor. The Traction Motor would now be regulated by a relatively simple on/off switch.

Additionally, in order to simplify experimentation purposes, an Internal Combustion Engine or ICE will not be included in the current experimental setup (as shown in Figure 2.4), which is how the GTD was tested previously. The purpose of this research is to find a more appropriate, i.e. more efficient, $3 \mathrm{I} / \mathrm{O}$ system that could function the same way, mechanically, using a similar setup.

\subsubsection{Standard Automotive Differentials}

In order to understand better how a GTD functions it would be helpful to understand how a Standard Automotive Differential (SAD) functions. An SAD, as seen in Figure 2.6, is a 3 I/O system used in automobiles to transfer power from the transmission to the drive wheels of the vehicle [25].

One example of the many SADs available can be seen on any half-ton truck or early model full-size SUV. The SAD for these vehicles would be located in the center of the vehicle's rear axle (also called the "pumpkin"). From Figure 2.6, it is easily noted that an SAD has 3 I/Os (an input from the drive-shaft and two outputs, one to each rear wheel). The SAD has been in use for over a century and almost all modern vehicles implement some form of it in their drivetrain. 


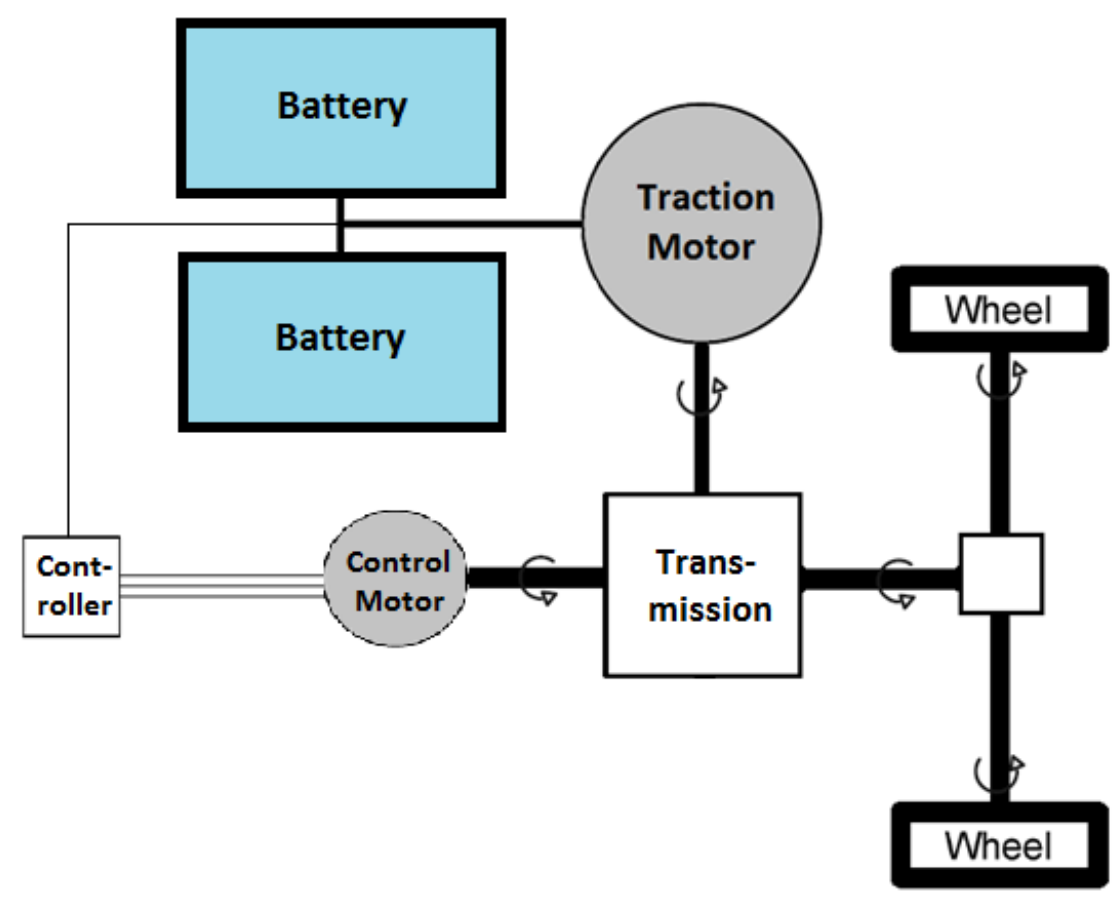

Figure 2.5: Modified Powertrain Layout of the Toyota Prius

\subsubsection{Gleason-Torsen Differentials}

The Gleason-Torsen Differential (GTD) is just one type of the many types of automotive differentials. The GTD, as used in automobiles, is a single-input, dual-output mechanical device (See Figure 2.7). It receives input power from the transmission and directs this power to one or both of the drive wheels. The gear labeled "ring gear" functions as the input, while the two separate "axle shafts" function as the two outputs to provide power to move the vehicle. As can be seen from Figure 2.7, this type of differential uses a series of different types of gears and, due to their specific configuration and functionality, is commonly referred to as a "limited slip" differential [26].

As was mentioned, the GTD was the subject of preliminary research that led to the current research described in this work. The GTD was tested previously for research in the following way: instead of using it as a single-input dual-output device, it was tested as a single-output dual-input device; in other words, it was tested in reverse. The two axle shafts functioned as the inputs while the ring gear functioned as the output. Testing a differential in such a way allows the speed of the 


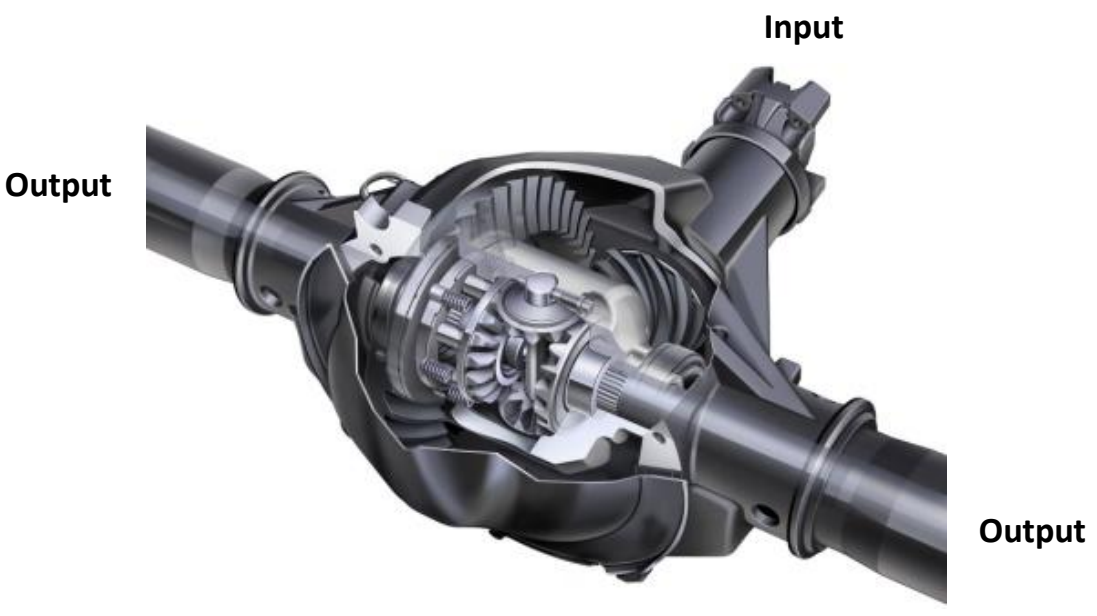

Figure 2.6: Standard Automotive Differential

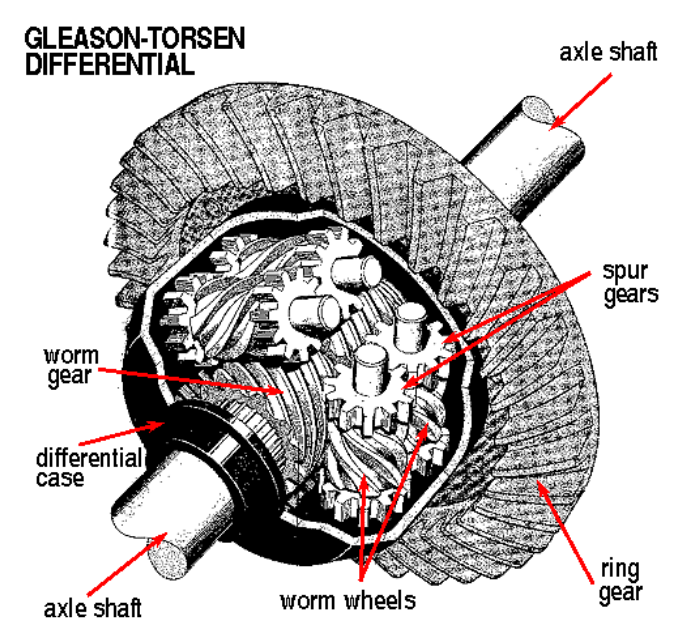

Figure 2.7: Gleason-Torsen Differential

two inputs to vary independent of each other, while contributing to the speed of the output through the following relationship:

$$
\omega_{\text {out }}=\frac{\omega_{i n 1}+\omega_{i n 2}}{2}
$$

where $\omega_{\text {out }}$ is the output speed, $\omega_{i n 1}$ is the first input speed and $\omega_{i n 2}$ is the second input speed. For the purpose of simplicity in this research, we will hereafter refer to the dual-inputs and single- 
output of this system and others through this same Equation (2.2), but modified as seen in Equation (2.3):

$$
r p m C=\frac{r p m A+r p m B}{2}
$$

where $A$ and $B$ represent the two inputs and $C$ represents the output. This relationship can be derived using fundamental gear analysis together with a method known as "The Tabular Method" [27]. This relationship, as was derived for the system tested in this research, will be explained in greater detail later in this work.

Although this type of differential worked in previous testing to demonstrate functionality, it is not the most efficient type of system for this particular application [28]. A more efficient, but similar system is needed for practical application under this type of testing scenario.

\subsubsection{Planetary Gear Sets}

Another type of 3 I/O device is known as the Planetary Gear Set (PGS). A PGS, as seen in Figure 2.8, can also be used with two I/Os acting as inputs and the third I/O acting as the output [29]. The three different I/Os are labeled in Figure 2.8 as "Sun Gear", "Ring Gear" and "Carrier", where the carrier is the combined rotational I/O for the "Planet Gears."

Planetary Gear Sets are used extensively in automatic transmissions for passenger vehicles, but are not used as $3 \mathrm{I} / \mathrm{O}$ systems in most of these applications. Instead, one of the I/Os is held at zero angular velocity, while the other two move at an unchanging predetermined ratio. This is the essence of how the internal components of automatic transmissions work. Multiple gear ratios (or speeds) are obtained through a series of more than one PGS that only function effectively as single degree of freedom mechanisms.

Within the family of the PGS, there are a variety of types and/or configurations used. Two common types of PGSs are the Simpson PGS and the Ravigneaux PGS [14]. These types are similar to two standard PGSs placed in series, but share the same sun or planet gears, depending on the type and requirement of the system. There are also PGSs consisting of multiple sets of planet gears, commonly referred to as a Compound PGS. These variations of the PGS allow distinctly different gear ratios and expand the design space of the system. Some versions can even allow 


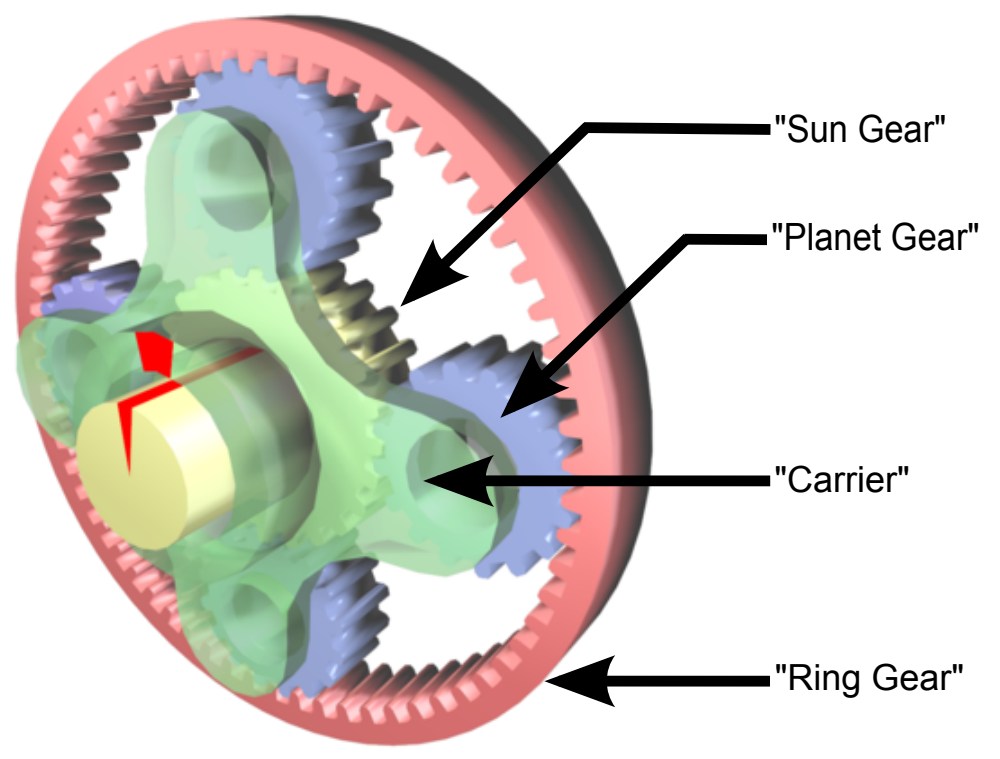

Figure 2.8: Typical Planetary Gear Set

for more than 3 I/Os, like the Toyota Prius described earlier. This work will focus only on 3 I/O systems. These variations are mentioned in this work to cover more of the variety of available types of $3 \mathrm{I} / \mathrm{O}$ systems in use and available for application in automobiles.

\subsubsection{Variable-Geometry CVT}

Another type of $3 \mathrm{I} / \mathrm{O}$ system not yet discussed is also another type of CVT known as the Variable-Geometry CVT (VGCVT). This type of CVT, as seen in Figure 2.9, is not commonly used in automobiles, but has been used in other similar applications. One such application would be its use and development in bicycles [30]. The VGCVT uses a set of sprockets and a chain to transfer power. These sets of sprockets, as seen in Figure 2.9, expand to enlarge its diameter and change its effective gear ratio. Although this type of CVT was in development several years ago and would not be applicable in higher-powered vehicle applications, it does provide some additional insight as to the $3 \mathrm{I} / \mathrm{O}$ solutions that are currently available. 


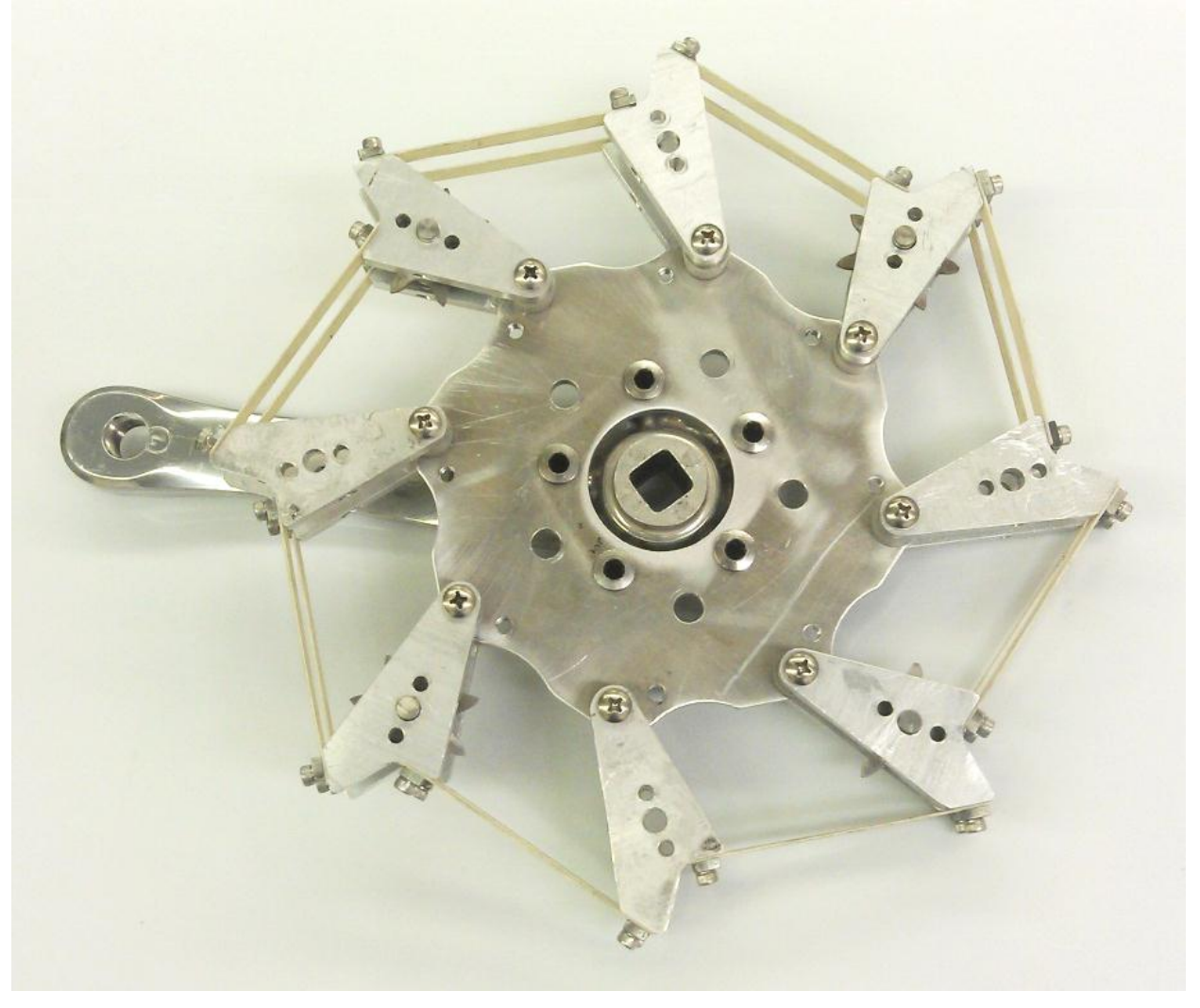

Figure 2.9: Variable-Geometry or Pivot Arm CVT

\subsubsection{Hydrostatic CVT}

The Hydrostatic Continuously Variable Transmission (HCVT) is another type of $3 \mathrm{I} / \mathrm{O}$ device that can be used to vary the gear ratio requirements of a system. This device, as seen in Figure 2.10, regulates high-pressure hydraulic fluid through the use of swash plates, where the red arrow indicates the location of one of the swash plates [31]. In an HCVT, input power turns a pump, which converts the rotational motion to fluid motion, and then a motor converts this fluid motion back to rotational motion. The conversion rate between the hydraulic fluid and the rotational motion is controlled by the swash plates located inside of the input and output pumps. The control of the swash plates is what constitutes the third I/O.

This type of device is commonly used in industrial farm equipment and other low speed, but high torque applications. Although it is capable of transferring high power, its efficiency is not good enough to render it useful in standard automobile applications [31]. 


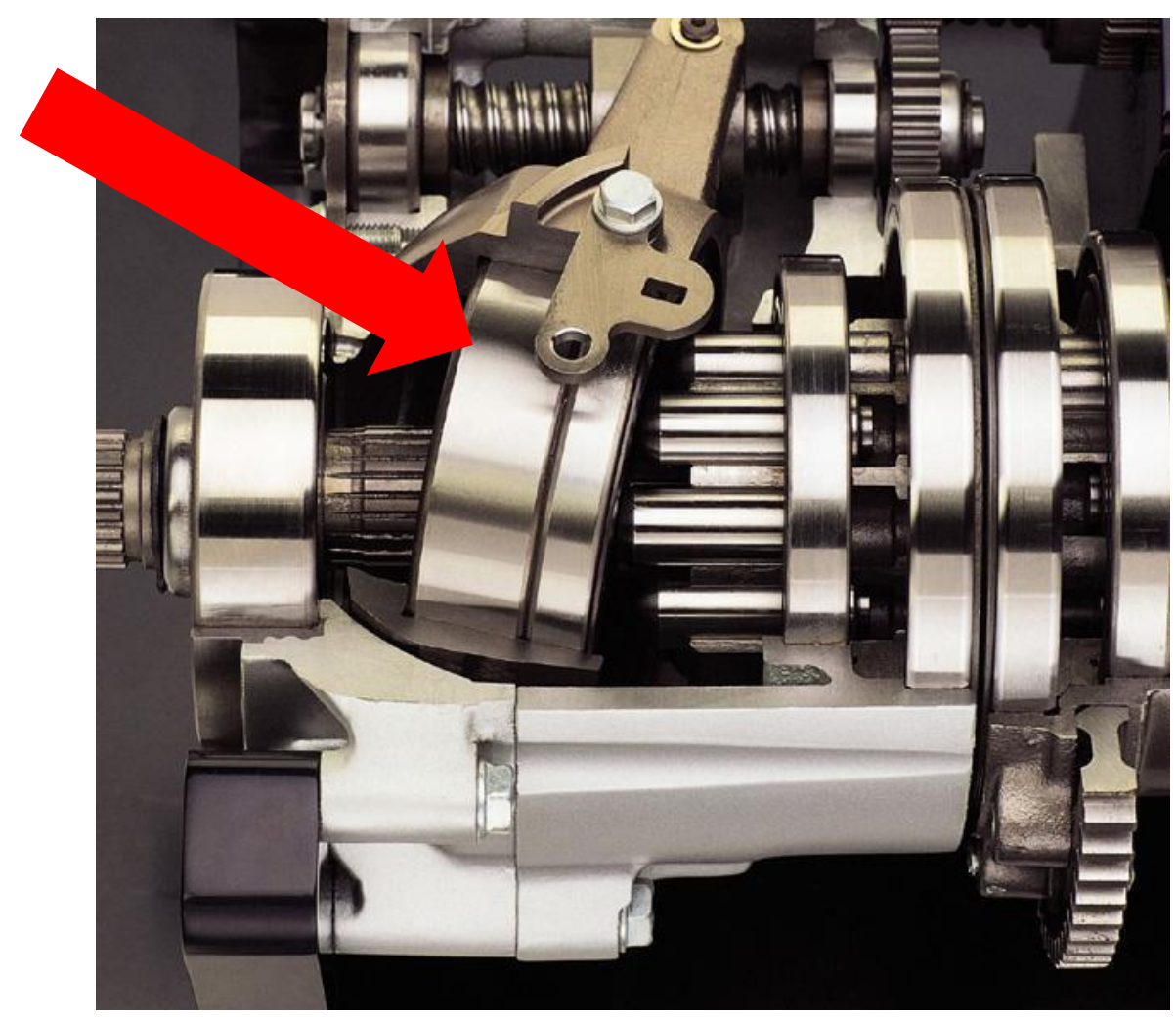

Figure 2.10: Exposed View of a Hydrostatic CVT

\subsubsection{Electric CVT}

The Electric Continuously Variable Transmission (ECVT) can also be classified as a 3 I/O system. An ECVT functions just like any other CVT in that it provides a continuous ratio change by varying one of the inputs, or controller, as seen in Figure 2.11. The ECVT provides this ratio change by increasing or decreasing the field of a DC electric motor and/or generator. It, therefore, does not require mechanical motion as an input to create the ratio change, but relies entirely on what is known as "field weakening" to provide the system or vehicle with the required gear ratio.

Although this system has great potential and has been in wide use for nearly a century (commonly know as the Ward Leonard System) it also suffers from significant efficiency loss as power typically must be transferred from mechanical energy to electrical energy, and then back to mechanical energy. At the same time, it may present itself as a potential solution in the near future and is still under investigation. For more on the potential of this type of system for use in 


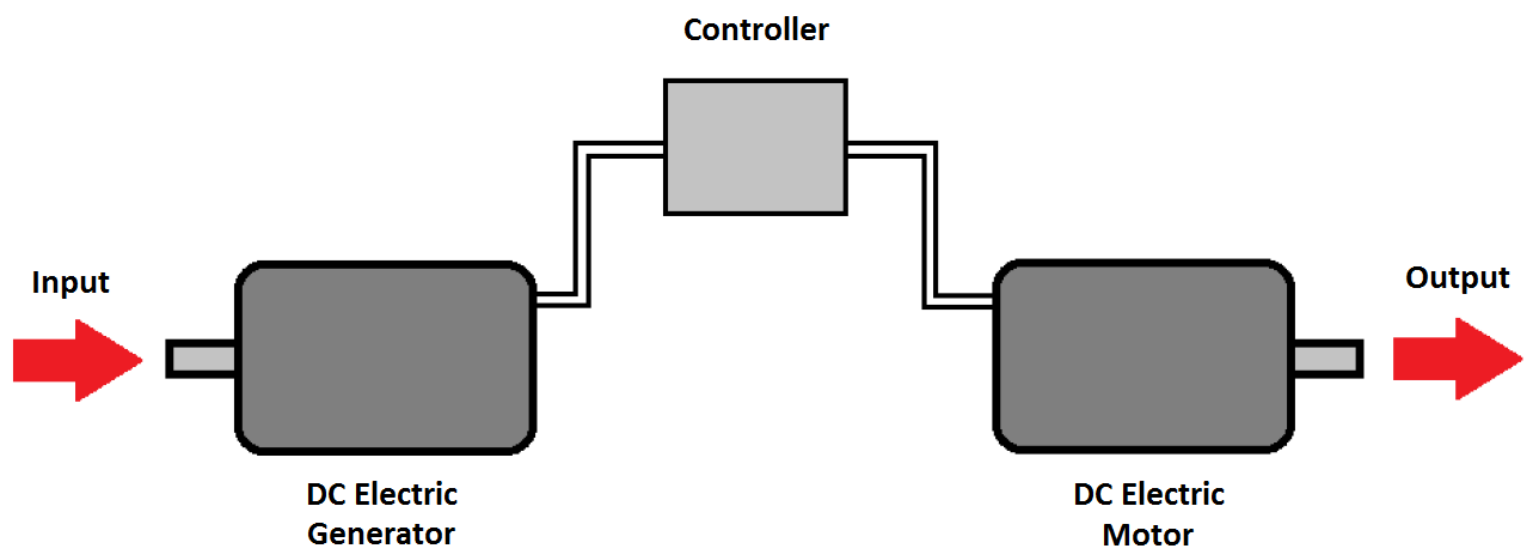

Figure 2.11: Simplified Version of an Electric CVT

automotive applications refer to An Investigation of the Ward Leonard System for use in a Hybrid or Electric Passenger Vehicle by Cody Telford [32].

\subsubsection{Planetary Differentials}

A Planetary Differential (PD), as seen in Figure 2.12, is yet another $3 \mathrm{I} / \mathrm{O}$ device, where the two large outer gears could act as two inputs and the shaft, which is rigidly connected to the carrier, could act as the output. A PD is very similar to a PGS in that it has a sun and planet gears. However, instead of having a ring gear like the PGS, the PD has a second set of planet gears and second sun gear. The "two larger outer gears" referred to in Figure 2.12, for this particular PD, are rigidly connected to the two sun gears of the system and the "carrier" is rigidly connected to, and houses, both sets of planet gears. A more detailed visualization and explanation of how a PD works will be covered later in this work.

This type of mechanical system has many advantages over other $3 \mathrm{I} / \mathrm{O}$ devices. Table 2.2 shows many of the advantages and disadvantages of a PD compared to the other $3 \mathrm{I} / \mathrm{O}$ devices that have been discussed. As can be seen from Table 2.2, the PD has an advantage in all categories other than its availability as an "off-the-shelf" product. As this system is used more and more in vehicle applications, it is anticipated that it will become all the more available. Additionally, construction of the PD for this research was fairly inexpensive due to the high availability of the majority of its components. 


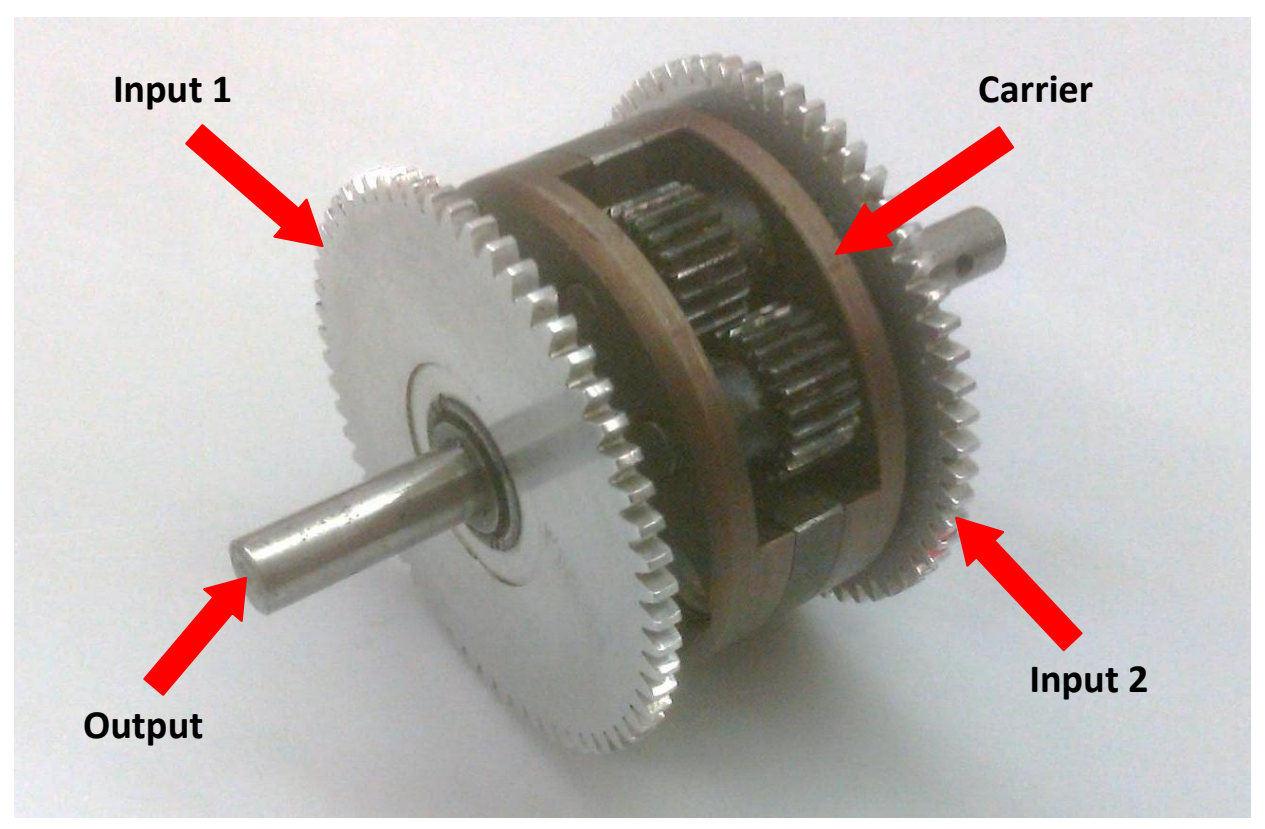

Figure 2.12: Planetary Differential

Table 2.2: Comparison of Various 3 I/O Systems

\begin{tabular}{|c|c|c|c|c|c|c|c|}
\hline Parameter & SAD & GTD & PGS & VGCVT & HCVT & ECVT & PD \\
\hline Compactness & Low & Low & High & Medium & Low & Low & High \\
\hline Optimization & Low & Low & Medium & Low & High & Medium & High \\
\hline Efficiency & Medium & Medium & High & High & Low & Low & High \\
\hline Load Capacity & High & High & High & Low & High & High & High \\
\hline Availability & High & High & Medium & Low & High & High & Low \\
\hline Design Versatility & Medium & Medium & Low & Low & High & High & High \\
\hline
\end{tabular}

\section{Design Versatility of a Planetary Differential}

One of the most pertinent of the advantages of a PD over other systems, as seen in Table 2.2 is its "Design Versatility". Design versatility, as defined in this research, is the physical freedom or geometric versatility that a designer would have in designing the size and location of each of its meshing pairs of gears.

A PD, for example, is made entirely from spur-type gears allowing each of the gears to be any given size depending on the gear ratio needed. A PGS, SAD or GTD, on the other hand, are limited in gear ratio selection by their respective ring and bevel gears [24]. For this reason the PD can be a better choice when designing a system with versatility of available gear ratios. 


\subsection{Previous Research}

As was mentioned, preliminary research has been accomplished by Wells and Groen in testing a GTD (in reverse) as a dual-input, single-output device for use as a CVT [3] [4]. This preliminary work showed that a differential of this type could be used as a CVT for automotive applications to replace a portion of the functionality of expensive electronic controllers. However, it also proved that a GTD, as used in this application, is very inefficient due mostly to the nature of the device being a "limited slip" mechanism. This inefficiency was just one of the many problems identified during previous research. This section will explain previous tests that were performed on the GTD along with inferences and recommendations gathered from this previous research.

\subsubsection{Tests Performed}

This subsection lists and explains some of the tests that were performed while investigating the GTD as a potential solution [28]. The following is a list of pertinent tests performed:

- Test $1 \mathrm{~s}$

- Test $2 \mathrm{~s}$

- Test 10

- Test 20

- Crossover

where Tests $1 \mathrm{~s}$ and $2 \mathrm{~s}$ were tests performed with both inputs applying power in the same direction and Tests 10 and 20 were performed with the two inputs applying torque in opposite directions. The difference between Tests $1 \mathrm{~s}$ and $2 \mathrm{~s}$ are simply the behavior of the two inputs as they rotated in the same direction. The difference between Tests 10 and 20 can be described similarly, but with the two inputs rotating in opposite directions. The Crossover test was performed with one input running at a constant velocity and the second input starting in the opposite direction and then crossing over to run in the same direction as the first input. Each of these tests performed on the GTD proved the validity of Equation (2.3) along with the following equation describing the power relationship of the system [24] [27]: 


$$
T_{A} * r p m A+T_{B} * r p m B+T_{C} * r p m C=0
$$

again, where $\operatorname{rpmA}, \operatorname{rpmB}$ and $\operatorname{rpm} C$ represent values of Input $\mathrm{A}$, Input $\mathrm{B}$, and Output $\mathrm{C}$; and the values of $T_{A}, T_{B}$ and $T_{C}$ correlate to torque seen at Input A, Input B and Output C, respectively. It should be noted that Equation (2.4) assumes zero efficiency loss.

The torque relationship for the GTD can be derived from Equations (2.3) and (2.4) for three specific scenarios:

- $\operatorname{rpm} A=0$

- $\operatorname{rpmB}=0$

- $\operatorname{rpm} C=0$

In the first scenario, Equations (2.3) and (2.4) are simplified yielding Equations (2.5) and (2.6), which can further be simplified by plugging Equation (2.5) into Equation (2.6) to yield Equation (2.7).

$$
\begin{gathered}
r p m C=\frac{r p m B}{2} \\
T_{B} * r p m B+T_{C} * r p m C=0 \\
2 T_{B}=-T_{C}
\end{gathered}
$$

Following the same process for the other two scenarios yields two additional relationships seen in Equations (2.8) and (2.9).

$$
\begin{gathered}
2 T_{A}=-T_{C} \\
T_{A}=T_{B}
\end{gathered}
$$


These three scenarios are only a few of many possible scenarios. However, the torque relationship that must hold to satisfy equilibrium conditions for any mechanical system similar to the GTD under all conditions is shown in Equation (2.10):

$$
T_{A}+T_{B}+T_{C}=0
$$

where the sum of all torques acting on the system must be equal to zero, which again assumes zero loss of efficiency [24] [27].

\section{Tests $1 \mathrm{~s}$}

In Test 1s, see Figure 2.13, Input A (blue diamond markers labeled as "rpmA"), was held constant at $1000 \mathrm{rpm}$, while the second input, Input B (red square markers labeled as "rpmB") varied from 0 to $1000 \mathrm{rpm}$. It demonstrated that the GTD obeys Equation (2.3). This can be seen, in Figure 2.13, as Output C (green triangle markers labeled as "rpmC") varied from 500 to 1000 rpm. A graph of the torques seen at each of the I/Os for this test is also given, see Figure 2.14, to show that it also obeys Equation (2.10). Finally, Figure 2.15 shows the power relationship of the GTD during this test and demonstrates its compliance to Equation (2.4). Power for all 5 tests was calculated using Equation (2.1).

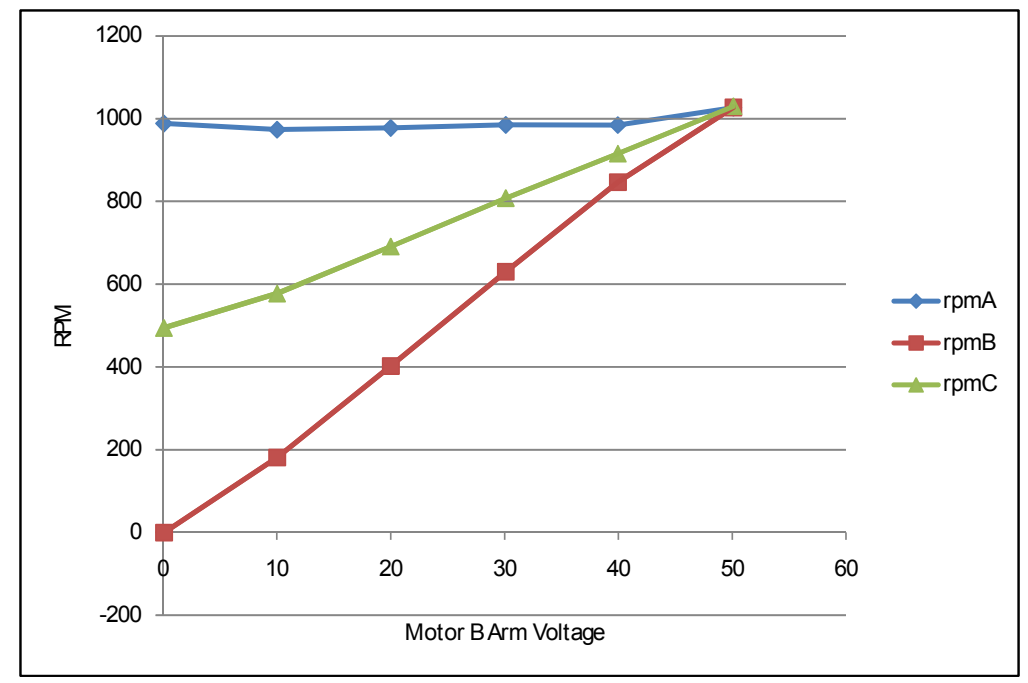

Figure 2.13: Test 1s - Angular Velocity 


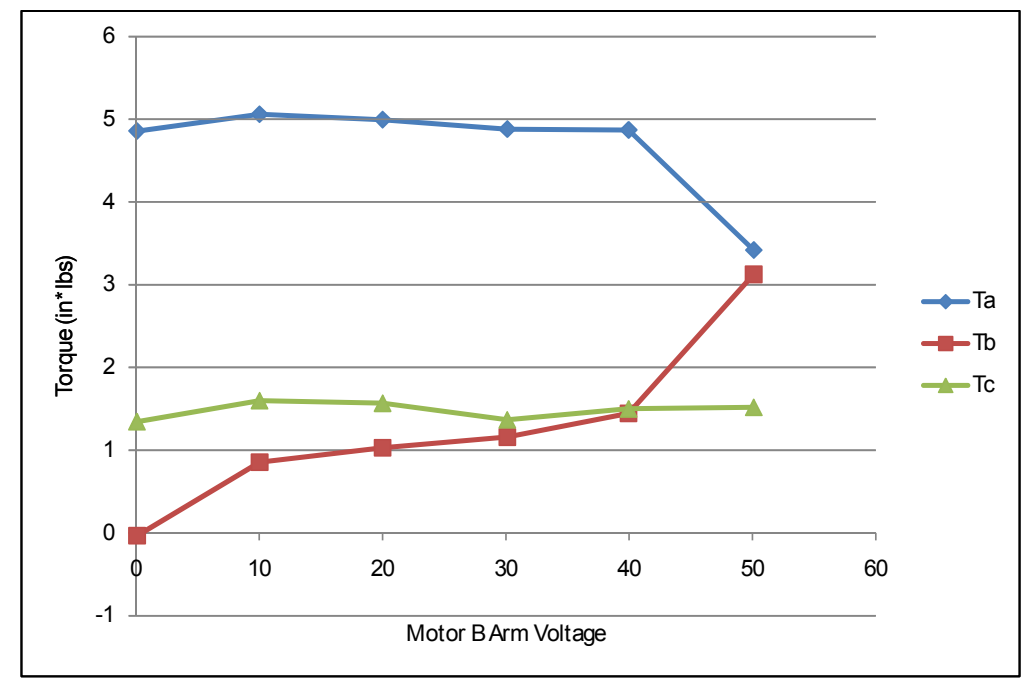

Figure 2.14: Test 1s - Torque

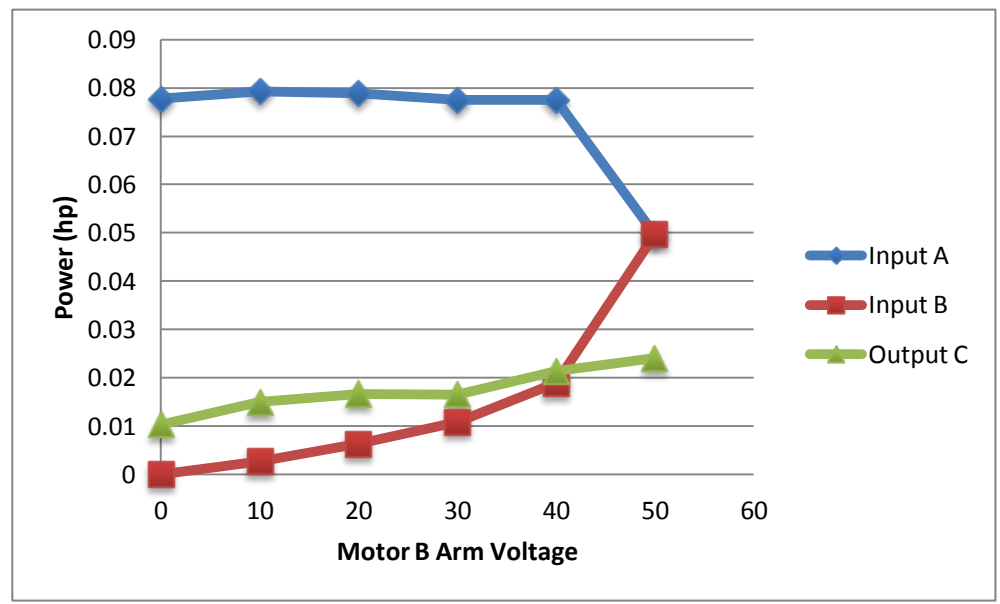

Figure 2.15: Test 1s - Power

\section{Tests 2s}

In Test 2s, Input A and B started from $0 \mathrm{rpm}$ and varied to 1000 and $500 \mathrm{rpm}$, respectively. This test also demonstrated that the GTD, again, obeys Equation (2.3). This result can be seen, in Figure 2.17, as Output $\mathrm{C}$ varied from 0 to $750 \mathrm{rpm}$. Graphs of measured torque and power are also shown in Figures 2.18 and 2.19. 


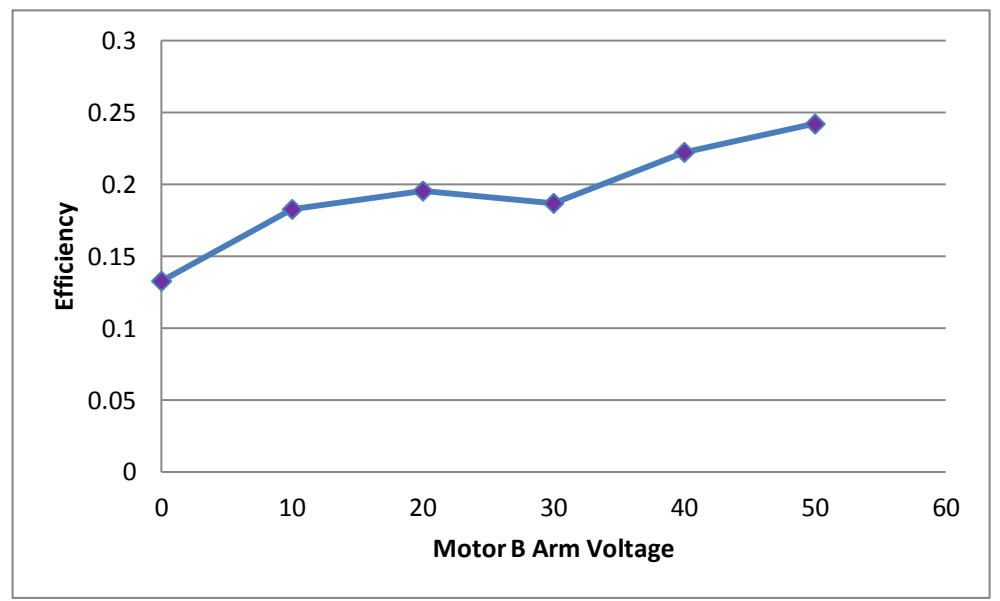

Figure 2.16: Test 1s - Efficiency

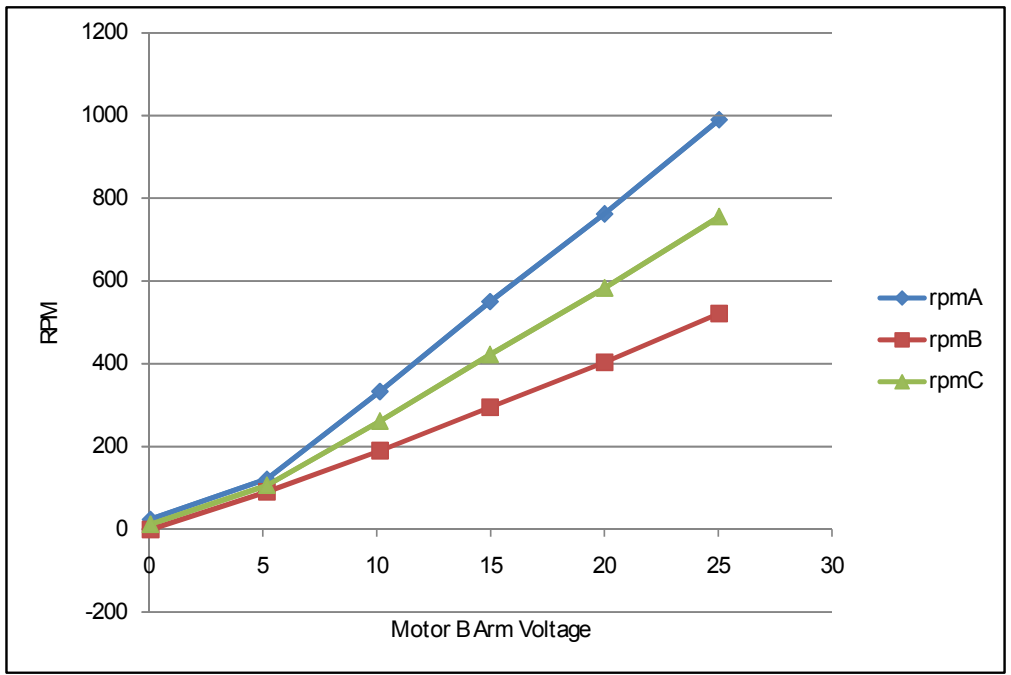

Figure 2.17: Test 2s - Angular Velocity

\section{Tests 10}

In Test 1o, Input A (blue diamond markers), was held constant at $1000 \mathrm{rpm}$, while the second input, Input B (red square markers) varied from 0 to $-1000 \mathrm{rpm}$. This test, like the previous two tests, demonstrated that the GTD, again, obeys Equation (2.3). This result can be seen, in Figure 2.21, as Output C (green triangle markers) varied from 500 to $0 \mathrm{rpm}$. Like in Test 1s, a graph of the torques seen at each of the I/Os for Test 1o is also given, see Figure 2.22, to show the torque that was measured during this test. Lastly, a graph of the power distrubution for this test is also shown in Figure 2.23. 


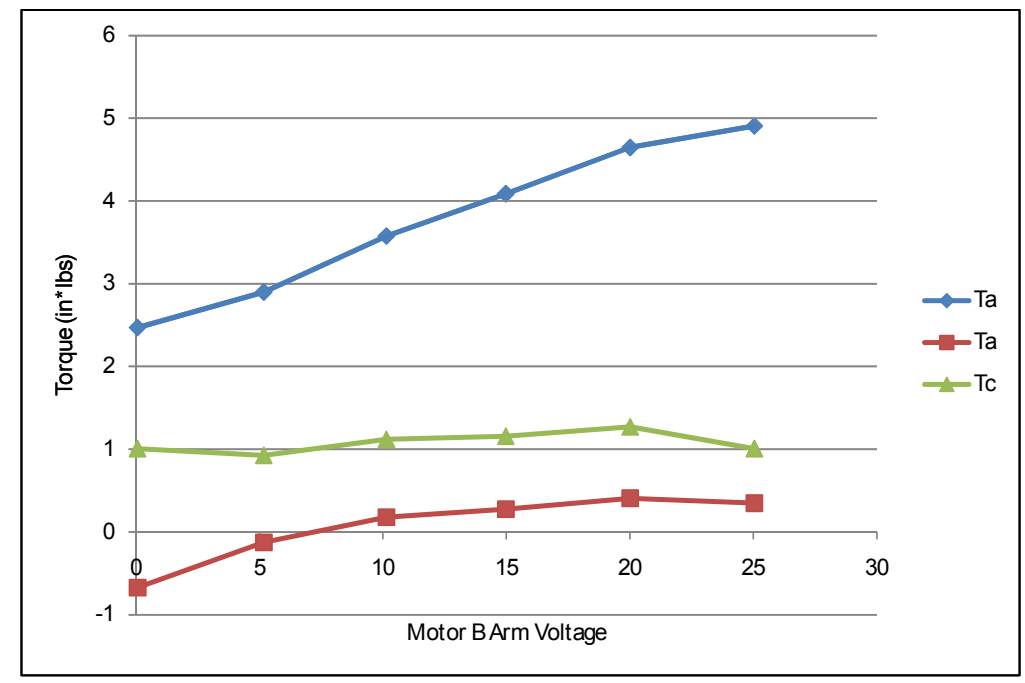

Figure 2.18: Test 2s - Torque

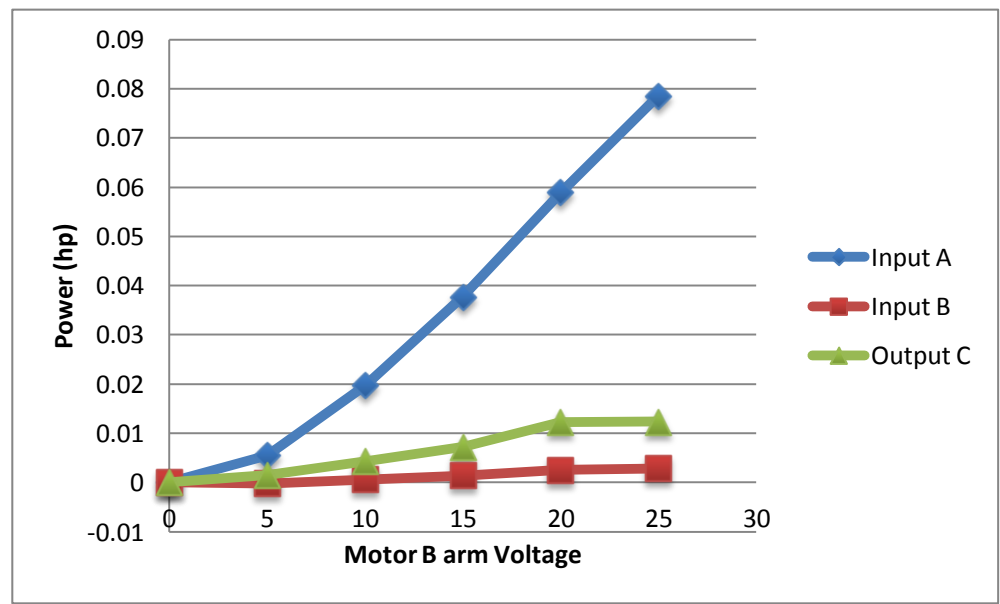

Figure 2.19: Test 2s - Power

\section{Tests 2o}

In Test 2o, Input A and B started from $0 \mathrm{rpm}$ and varied to 950 and $-550 \mathrm{rpm}$, respectively. This was but another demonstration that the GTD obeys Equation (2.3). This result can be seen, in Figure 2.25, as Output $\mathrm{C}$ varied from 0 to $200 \mathrm{rpm}$. Graphs of measured torque and power are also shown in Figures 2.26 and 2.27. 


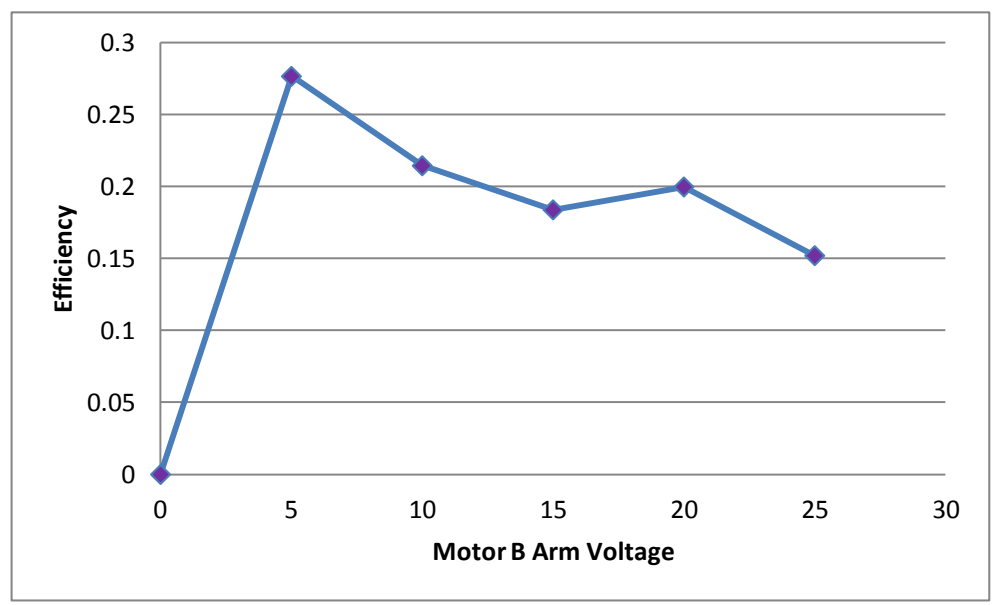

Figure 2.20: Test 2s - Efficiency

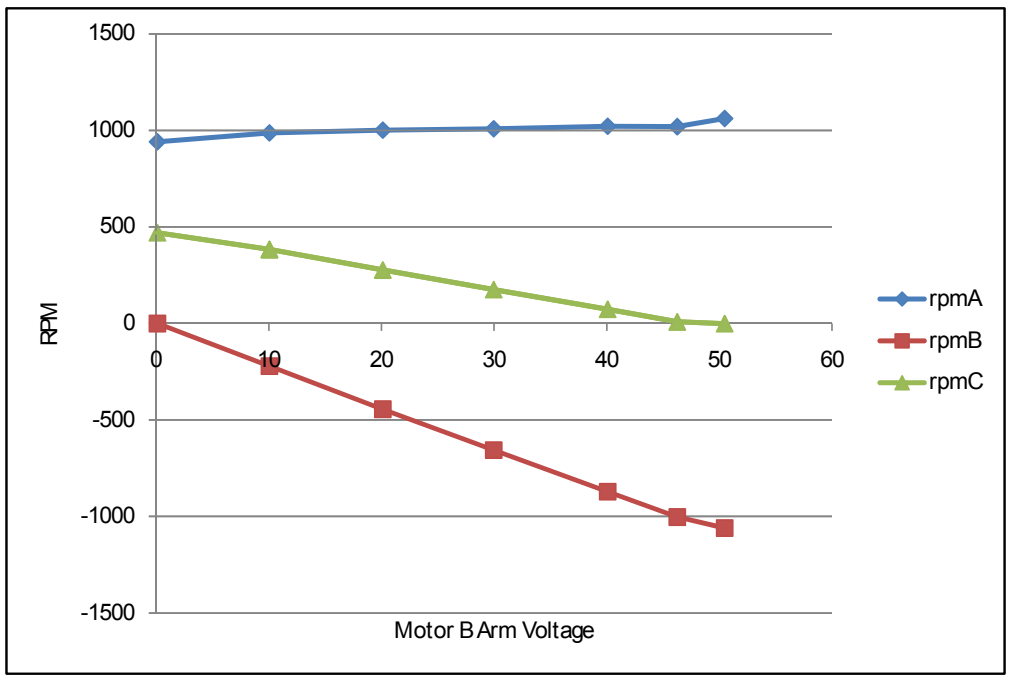

Figure 2.21: Test 1o - Angular Velocity

\section{Test Crossover}

Finally, in Test Crossover, Input A, was held constant at 600 rpm, while Input B varied from -1200 to $1000 \mathrm{rpm}$. This test was just one more that demonstrated the GTD's obedience to Equation (2.3). This result can be seen, in Figure 2.29, as Output C varied from -250 to $800 \mathrm{rpm}$. Graphs of measured torque and power are also shown in Figures 2.30 and 2.31. 


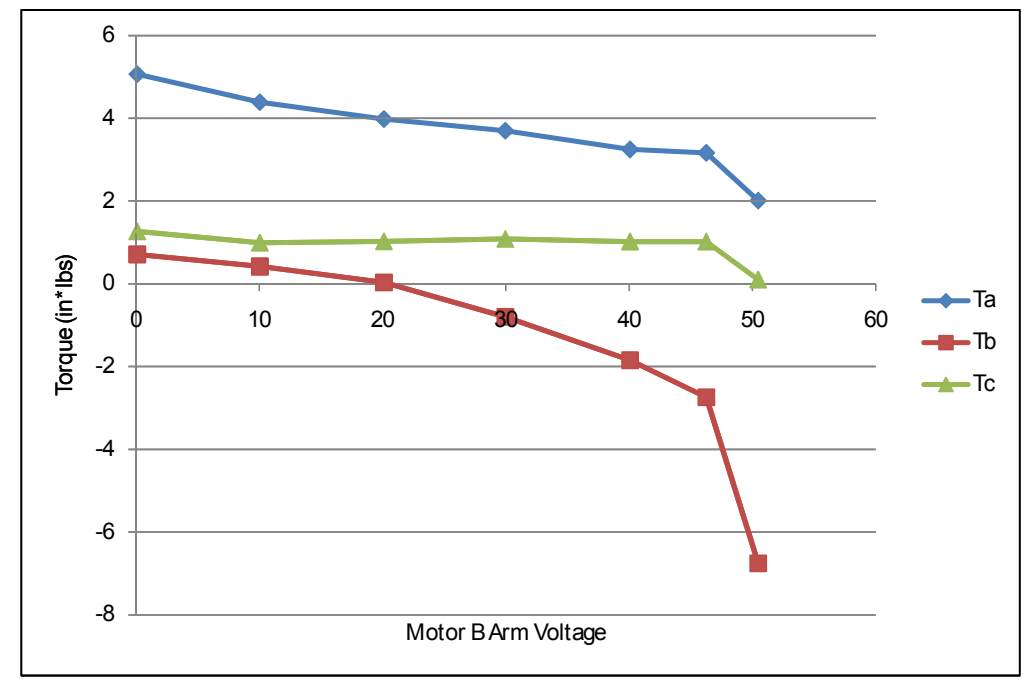

Figure 2.22: Test 10 - Torque

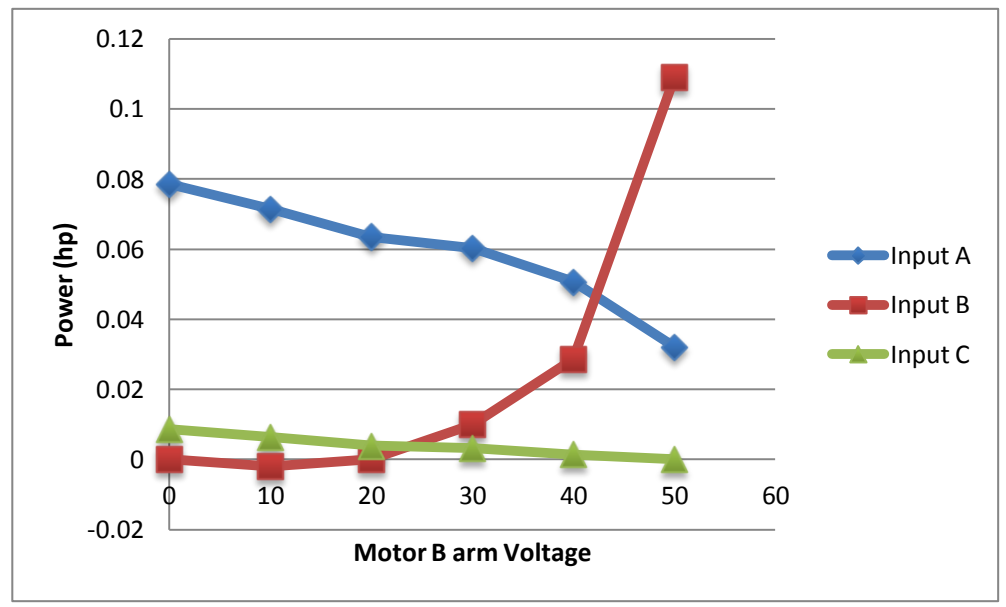

Figure 2.23: Test 1o - Power

\subsubsection{Efficiency}

Even though these tests demonstrated the capability of the GTD to function as a CVT, the GTD proved to be an inefficient solution. The mechanical efficiency of the GTD was determined from each of the five tests and averaged together. Efficiency for each of the tests was calculated using the following equation [24]:

$$
\eta_{\text {mech }}=\frac{P_{\text {out }}}{P_{\text {in }}}
$$




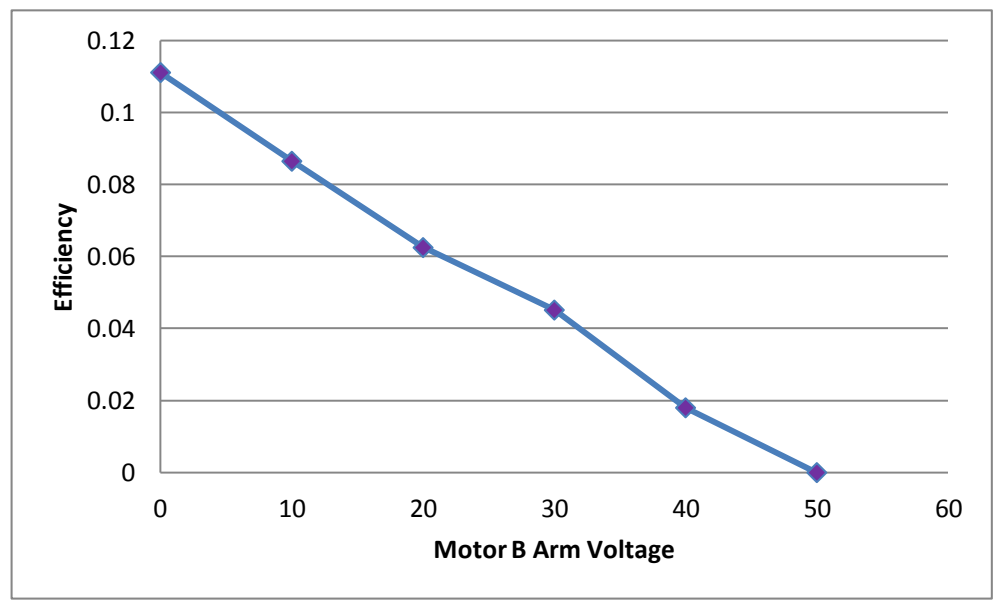

Figure 2.24: Test 1o - Efficiency

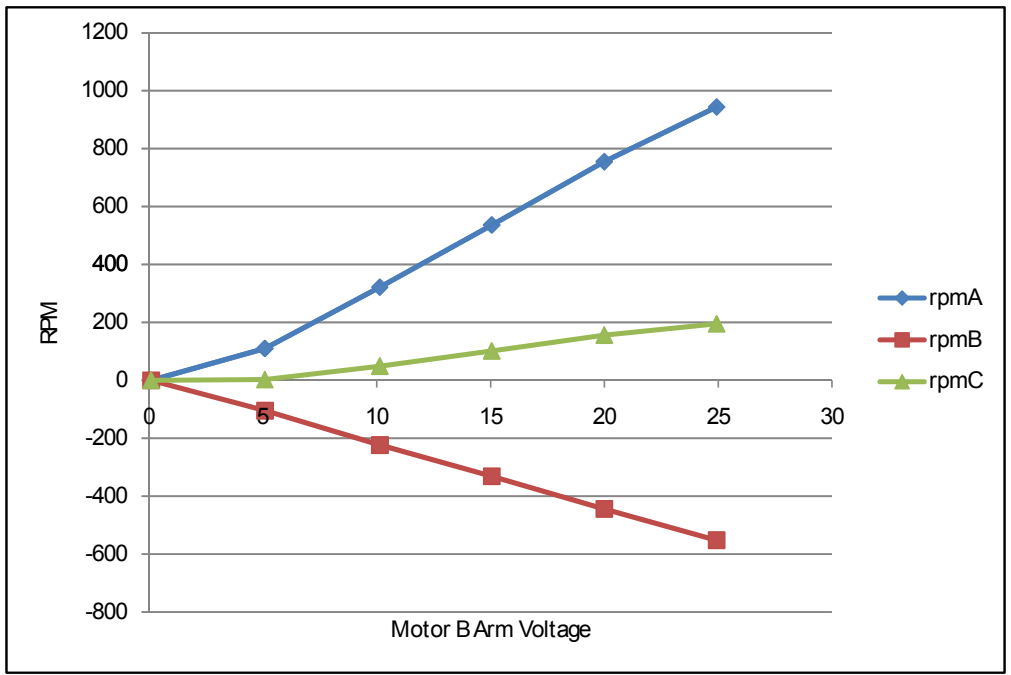

Figure 2.25: Test 2o - Angular Velocity

where $P_{\text {in }}$ is the product of torques and angular velocities, respectively, from Inputs A and B and $P_{\text {out }}$ is the same product, but from Output C. Equation (2.11) can, therefore, also be written as seen in Equation (2.12).

$$
\eta_{\text {mech }}=\frac{T_{C} * r p m C}{T_{A} * r p m A+T_{B} * r p m B}
$$

The calculated mechanical efficiency, as gathered from each of the tests performed on the GTD, can be seen in Table 2.3. Each column shows the lowest and highest efficiencies achieved 


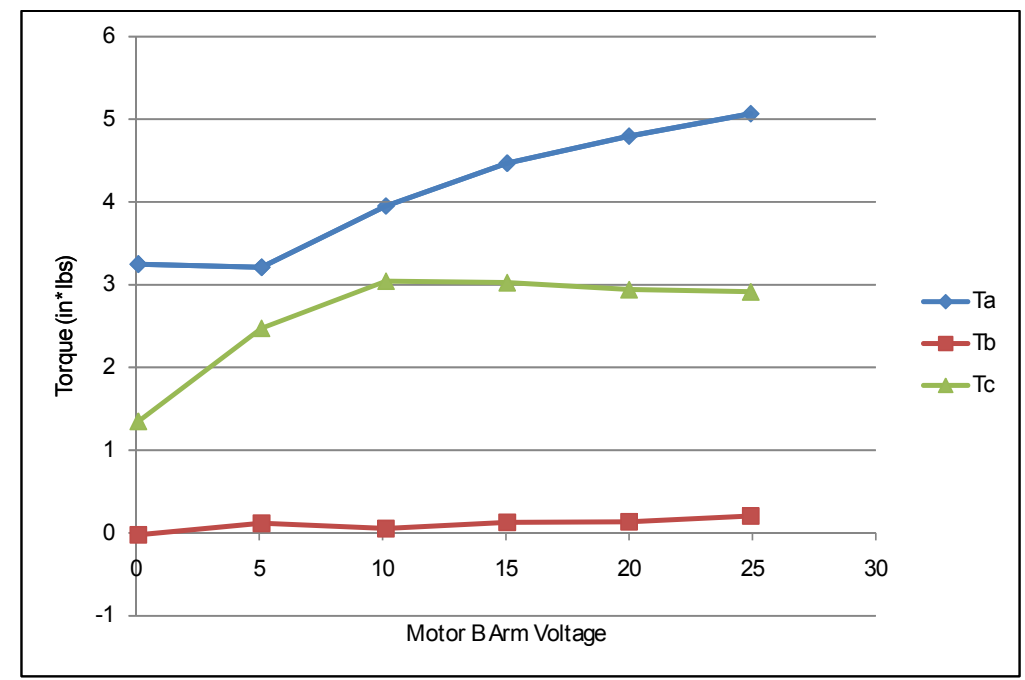

Figure 2.26: Test 2o - Torque

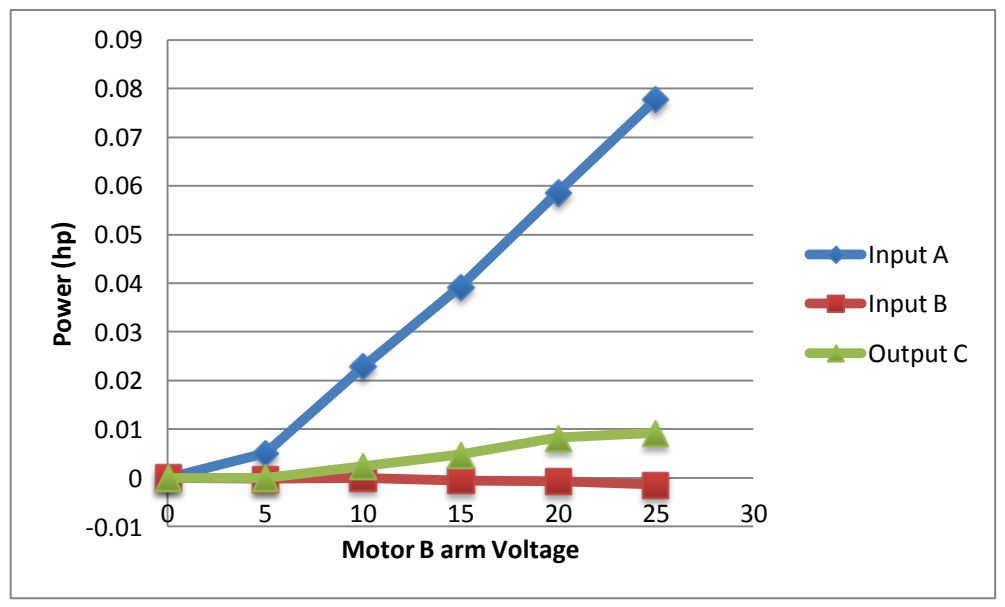

Figure 2.27: Test 2o - Power

for a single data point during each respective test along with an average of all data points for each of the tests. The bottom row shows averages from all 5 tests.

\subsubsection{Problems Identified}

During the tests that were performed to investigate the GTD, several problems were both anticipated before testing and/or discovered during testing by Wells and Groen [3] [4]. The following are a list of some of these problems: 


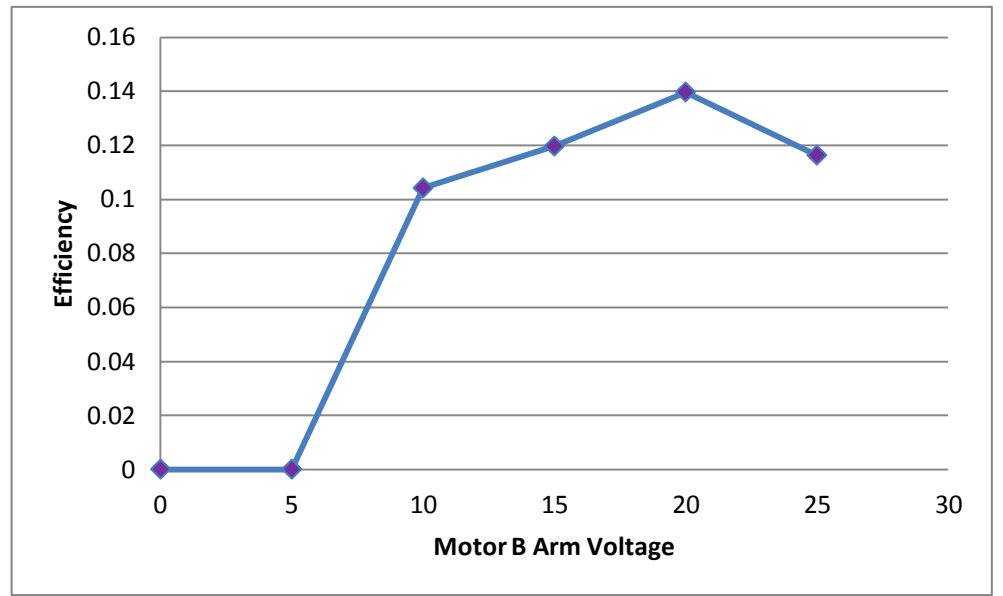

Figure 2.28: Test 2o - Efficiency

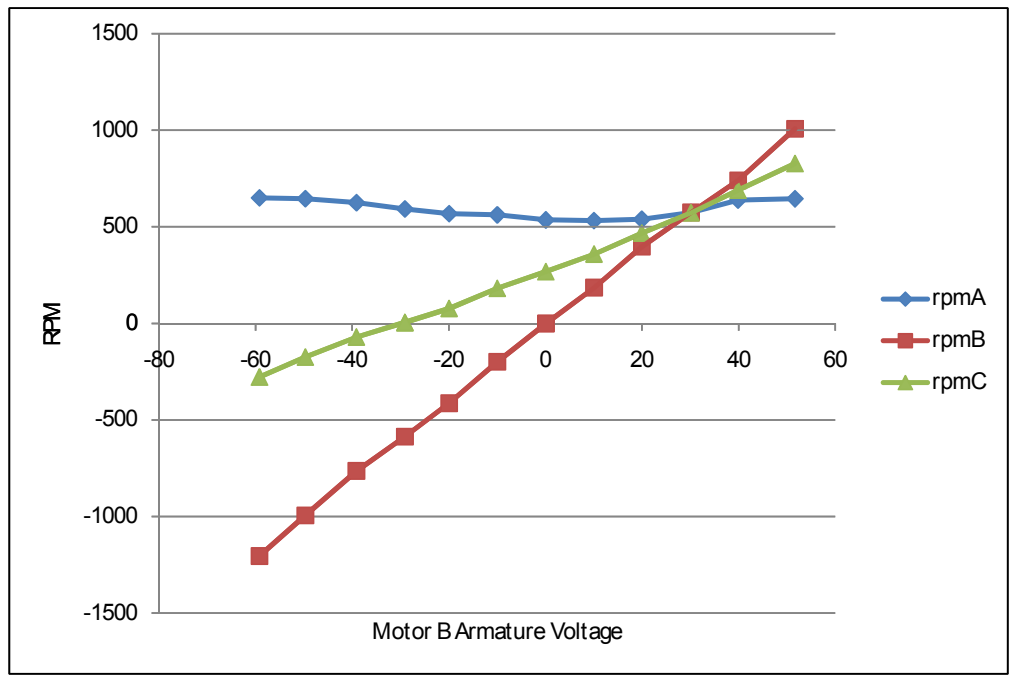

Figure 2.29: Test Crossover - Angular Velocity

- The system is a limited-slip-type differential.

- The previous use of the system was unknown.

- The system was not designed to be tested as such.

- The efficiency of the system was low.

- The system is back-drive-able.

- The system provides minimal gear ratio versatility. 


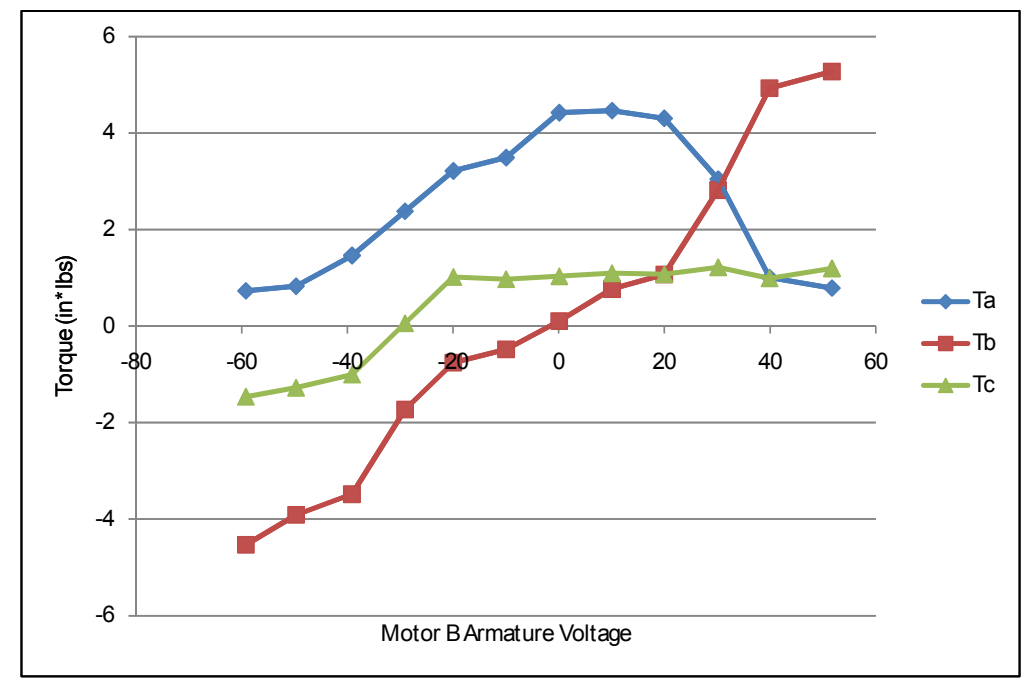

Figure 2.30: Test Crossover - Torque

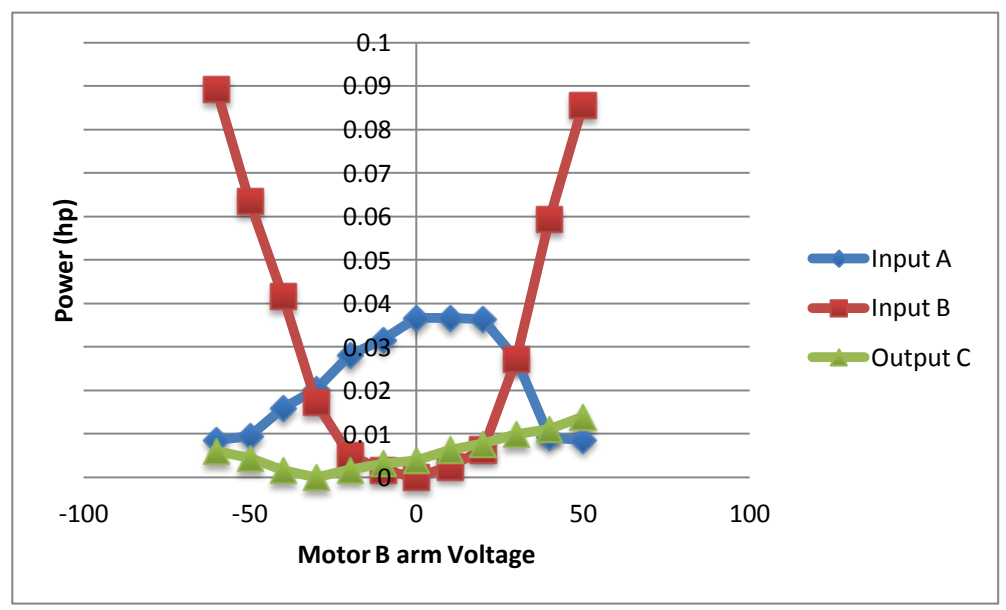

Figure 2.31: Test Crossover - Power

After examining these problems, it was determined that a new system ought to be chosen in place of the GTD to function in the same manner, but provide greater efficiency and solve the issue of back-drive-ability. Wells recommended a Planetary Differential be used together with some type of gear reduction device to be placed at Input B, or the location of the controlling motor [3]. The research done in this work will focus on testing a Planetary Differential as a new potential solution, while the issue of back-drive-ability will only be discussed briefly in this work, but more fully addressed as a separate topic in future research. 


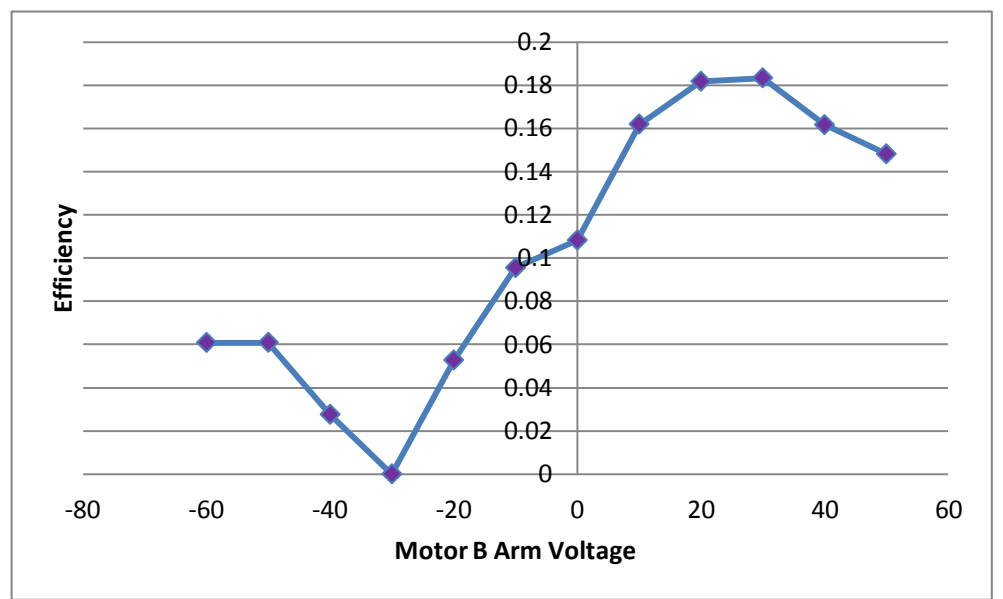

Figure 2.32: Test Crossover - Efficiency

Table 2.3: Efficiency of the Gleason-Torsen Differential

\begin{tabular}{|c|c|c|c|}
\hline Test Name & Low (\%) & High (\%) & Average (\%) \\
\hline 1s & 13.265 & 24.194 & 19.359 \\
\hline 2s & 15.199 & 27.654 & 20.529 \\
\hline 1o & 0 & 11.111 & 6.465 \\
\hline 2o & 0 & 13.968 & 11.995 \\
\hline Crossover & 0 & 18.333 & 10.354 \\
\hline Average & 5.693 & 19.052 & 13.740 \\
\hline
\end{tabular}




\section{CHAPTER 3. PLANETARY DIFFERENTIAL AS A POTENTIAL SOLUTION}

The purpose of this research is to investigate a Planetary Differential for use as a CVT, and specifically to determine its potential as an alternative solution. This will be done to discover if a PD can replace a portion of the functionality of electronic controllers in EVs, thus reducing their overall cost; also, to see if the PD is a more efficient solution than the previously-tested GTD.

\subsection{Physical Description}

The proposed potential solution, as seen in Figure 3.1, represents the 3 I/O system, consisting entirely of spur gear meshings, that was investigated and tested during the process of this research.

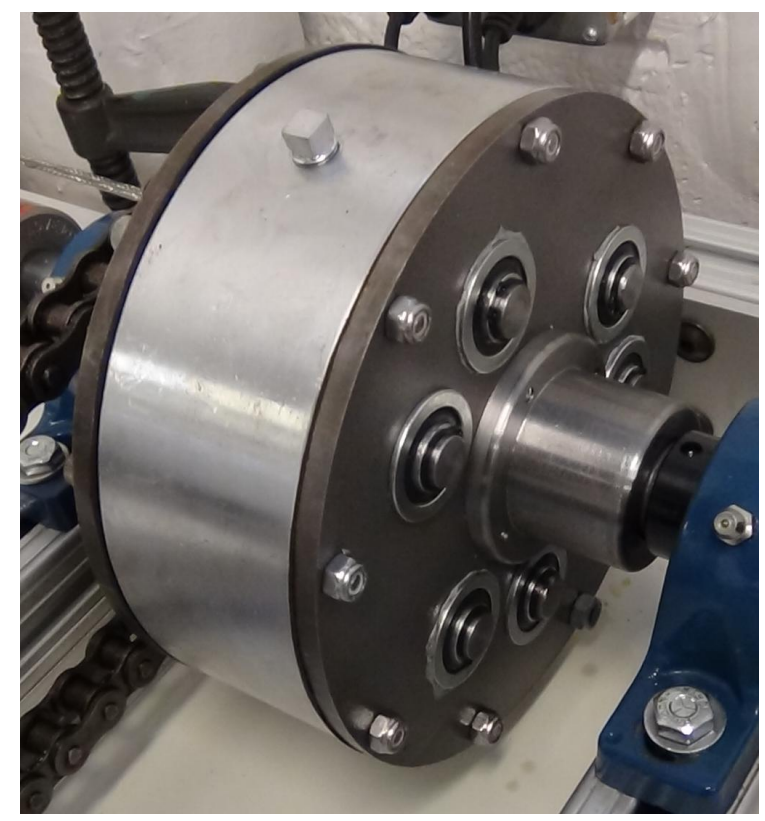

Figure 3.1: Planetary Differential 
A more informative view can be seen from Figure 3.2, showing multiple section views of a CAD drawing of the PD. The two Input Shafts A and B, labeled in Figures 3.2(d) and 3.2(b), are rigidly connected to Sun Gears A and B, labeled in Figure 3.2(c) and 3.2(a), and located between the two Carrier Plates, labeled in Figures 3.2(a) and 3.2(d), of the PD. These two Sun Gears are meshed with two separate sets of Planet Gears that are meshed with one another and axially fixed to the Carrier Plates. The Carrier Plates rotate, as if one plate, around the two Input Shafts, but are also rigidly connected to the Output Gear (a sprocket was used in place of a gear for the actual test setup).

This system was built under the author's direction by a team of students at Brigham Young University-Idaho. As it was designed specifically for the purpose of this investigative work, its construction is not perfect, but is otherwise fully-functional. It was designed and built to be capable of handling 50 foot pounds of torque at 3000 revolutions per minute, continuously, and requires SAE 75 weight oil, which is similar to that used in manual automotive transmissions.

\subsubsection{Components}

The PD used for this research was constructed from professionally-manufactured and inhouse manufactured or modified parts. The following is a list of all components that make up the PD:

- Sun Gears (2)

- Planet Gears (12)

- Input Shafts (2)

- Housing Supports (2)

- Housing Cover (1)

- Planet Shafts (6)

- Planet Shaft Bearings (12)

- Input Shaft Bearings (2) 


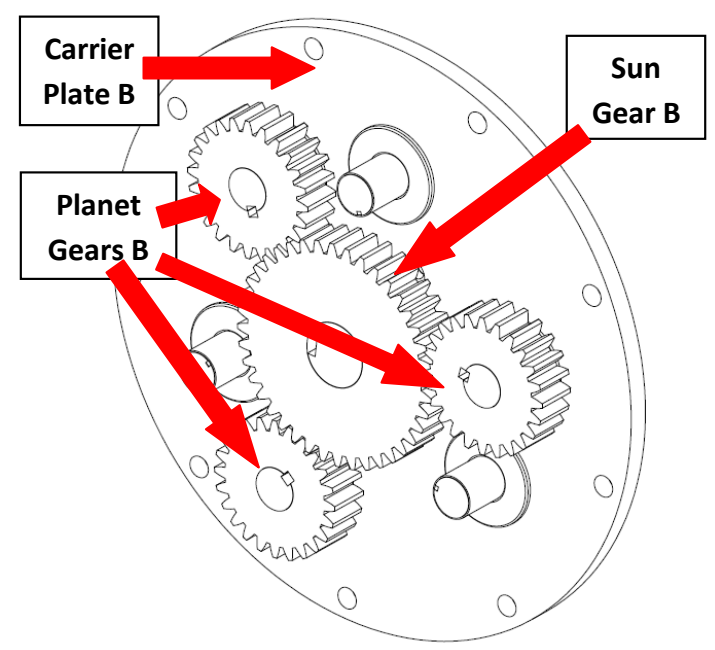

(a) Sun B, Planets B

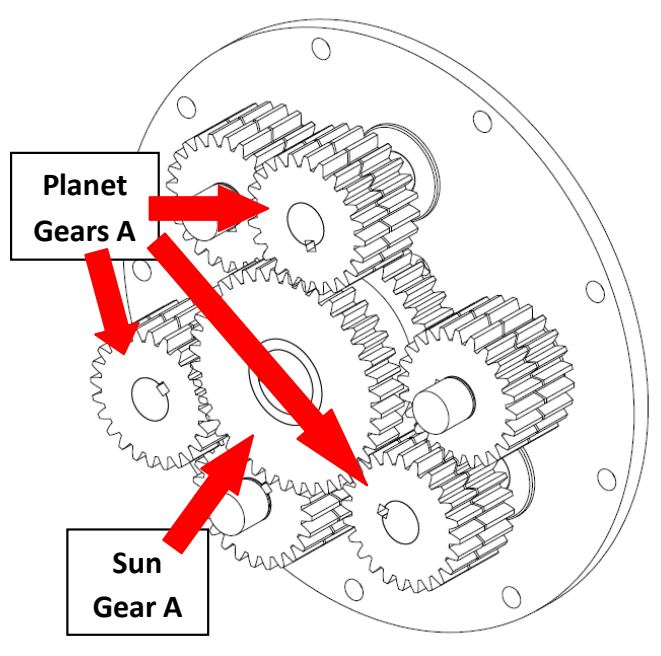

(c) Sun B and A, Planets B and A

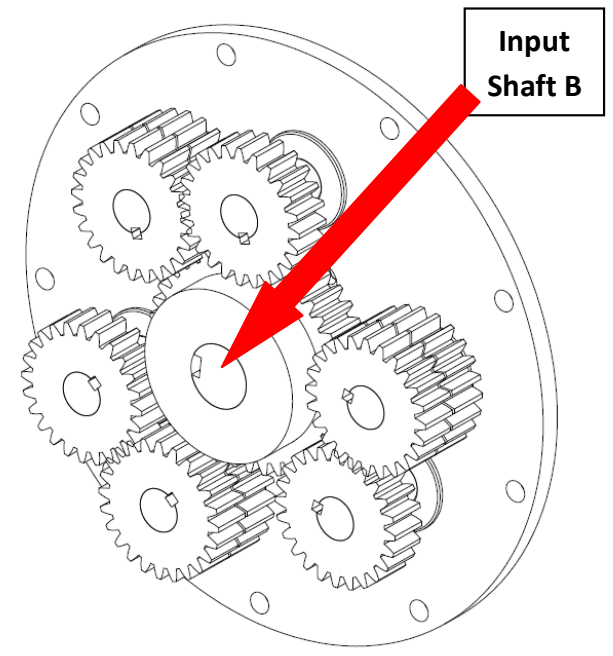

(b) Sun B, Planets B and A

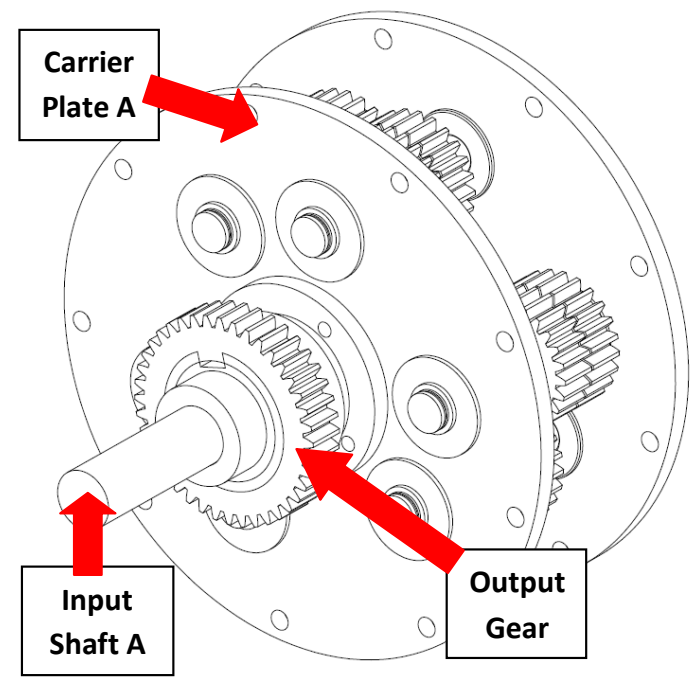

(d) Complete PD

Figure 3.2: Cutaway Views of the PD

- Various Retaining Clips

- Various Shaft Keys

- Various Seals and Gaskets 


\subsubsection{Dimensions}

The dimensions of the PD tested in this research are appropriate for its intended purpose. This system is, however, capable of being almost any needed size when designed for use in an automobile. A summary of this system's basic dimensions are give in Table 3.1.

Table 3.1: PD Basic Dimensions

\begin{tabular}{|c|c|c|}
\hline Part Name & Dimension & Value (in) \\
\hline Sun Gears & Outer Diameter & 4 \\
\hline Planet Gears & Outer Diameter & 2 \\
\hline Input Shafts & Diameter & 0.75 \\
\hline Carrier Plates & Outer Diameter & 7 \\
\hline Planet Shafts & Diameter & 0.5 \\
\hline
\end{tabular}

For more details on size of various parts of the PD and their locations with respect to one another refer to Appendix A at the end of this work.

\subsubsection{Gears}

All gears used in the PD were involute profile spur gears. These gears were purchased from a local manufacturer and fitted to work in the PD. As can be seen from Figure 3.2, on all six Planet Shafts two identical Planet Gears were fixed side-by-side to act as a single planet gear with twice the width. This was done simply because the price of two gears of half the required width was less expensive than one planet gear of the required width. Some minor radial misalignment of these two identical Planet Gears is believed to be the cause of some of the efficiency loss in the system. Future systems would be made with single sets of Planet Gears of the appropriate width.

\subsubsection{Material Properties}

The known general material of the main components that make up the PD, along with some other useful values, are listed in Table 3.2. The sum of weight of each of the listed parts multiplied by their respective quantities yields a total weight of $14.87 \mathrm{lb}$. Adding in a few more items unaccounted for in this table, including lubricant, would bring the estimated total weight to 
approximately $18 \mathrm{lb}$. The weight of this system, when manufactured for application in production vehicles, is anticipated to be heavier, but still lighter than other systems currently in use. The material choice for the various parts listed for the PD were selected based on the following criteria (and are listed in order of importance):

- Cost

- Availability

- Weight

- Material Strength

Since this is for testing purposes, cost and availability were the primary driving factors. In future testing, weight and strength of material will be more relevant as selection criteria.

Table 3.2: PD Part Material Properties

\begin{tabular}{|c|c|c|c|c|}
\hline Part Name & Material & Volume $\left(\mathrm{in}^{3}\right)$ & Weight $(\mathrm{lb})$ & Quantity \\
\hline Sun Gear & Steel & 3.568 & 1.012 & 2 \\
\hline Planet Gear & Steel & 0.744 & 0.220 & 12 \\
\hline Input Shaft & 1566 Steel & 2.062 & 0.584 & 2 \\
\hline Housing Support & 1020 Steel & 7.512 & 2.133 & 2 \\
\hline Planet Shaft & 1566 Steel & 0.640 & 0.181 & 6 \\
\hline Planet Shaft Bearing & 841 Bronze & 0.320 & 0.102 & 12 \\
\hline Input Shaft Bearing & Steel & 0.172 & 0.049 & 4 \\
\hline Housing Cover & 6061-T6 Alum. & 23.18 & 2.266 & 2 \\
\hline
\end{tabular}

\subsection{Governing Equations}

The behavioral motion for the PD, as seen by each of the I/Os, can be explained by using only a few equations. The first of these equations describes the angular velocity relationship between each of the I/Os and will be referred to as the velocity equation (3.1), which was given earlier as Equation (2.3) and is repeated here for convenience. 


$$
r p m C=\frac{r p m A+r p m B}{2}
$$

As was explained previously, the values of $\operatorname{rpm} A, \operatorname{rpmB}$ and $\operatorname{rpm} C$ correlate to Input $\mathrm{A}$, Input B and Output C, as seen in Figure 3.3, and are in units of revolutions per minute. As Inputs A and $B$ provide angular velocity to the $\mathrm{PD}$, the angular velocity seen at Output $\mathrm{C}$ is the average of both Inputs $\mathrm{A}$ and $\mathrm{B}$.

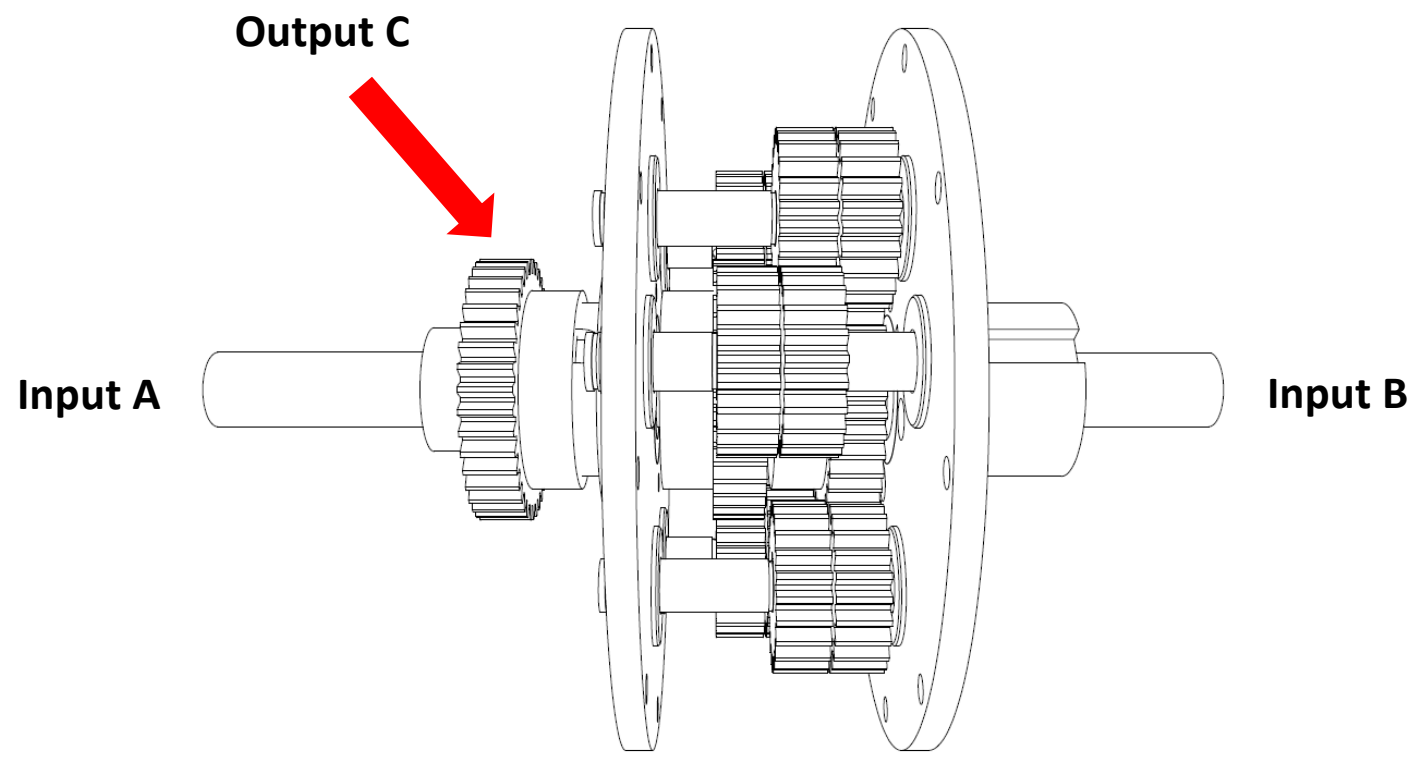

Figure 3.3: Planetary Differential with labeled I/Os

As was explained earlier in this work, this angular velocity relationship can be derived using fundamental gear relationships and a method known as "The Tabular Method" [27]. Before using the Tabular Method, the gear ratios between each set of meshed gears in the drivetrain of the system must be known. Gear ratios can be calculated by knowing the number of teeth on each of the gears. Table 3.3 shows gear teeth values of the PD tested in this research, while remembering that Input A is rigidly connected to Sun Gear A, Input B is rigidly connected to Sun Gear B and the Carrier for Planet Gears A and B is rigidly connected to Output C. Equation (3.2) shows how a gear ratio is calculated between two gears. 


$$
R=\frac{\text { Drive }}{\text { Driven }}
$$

Where $R$ refers to the gear ratio calculated, Drive refers to the gear that is providing the motion for the second gear and Driven refers to the gear that is being driven by the first gear. Table 3.4 shows gear ratios seen in the PD between each of the meshed gears going from Input A (Sun Gear A) to Input B (Sun Gear B). These gear ratios are all negative for the PD due to the fact that two external-type gears in mesh would rotate in opposite directions [27].

Table 3.3: Gear Properties of the PD

\begin{tabular}{|c|c|}
\hline Gear Name & Number of Teeth \\
\hline Sun A & 40 \\
\hline Planet A & 24 \\
\hline Planet B & 24 \\
\hline Sun B & 40 \\
\hline
\end{tabular}

Table 3.4: Gear Ratio Properties of the PD

\begin{tabular}{|c|c|c|c|}
\hline Drive Gear & Driven Gear & Gear Ratio & Simplified Ratio \\
\hline Sun A & Planet A & $-40: 24$ & $-5: 3$ \\
\hline Planet A & Planet B & $-24: 24$ & $-1: 1$ \\
\hline Planet B & Sun B & $-24: 40$ & $-3: 5$ \\
\hline
\end{tabular}

Table 3.5 shows an example of the Tabular Method in solving for the velocity of the third $\mathrm{I} / \mathrm{O}$ when velocities of the other two are known, while using the following equation to calculate the missing data:

$$
\omega_{\text {gear }}=\omega_{\text {carrier }}+\omega_{\text {gear } / \text { carrier }}
$$

where $\omega_{\text {gear }}$ refers to the angular velocity of the gear of that particular row, $\omega_{\text {carrier }}$ refers to the angular velocity of the Carrier for the Planet Gears and $\omega_{\text {gear/carrier }}$ refers to the angular velocity of the gear of that row with respect to the Carrier [27]. For the example in Table 3.5, Input A (Sun 
Gear A) has a known angular velocity of 200 RPM and Output C (Carrier for Planet Gears A and B) has a known angular velocity of 100 RPM.

Table 3.5: Example of using the Tabular Method

\begin{tabular}{|c|c|c|c|}
\hline Gear Name & $\omega_{\text {gear }}=$ & $\omega_{\text {carrier }}+$ & $\omega_{\text {gear } / \text { carrier }}$ \\
\hline Sun A & 200 & 100 & 100 \\
\hline Planet A & -66.67 & 100 & -166.67 \\
\hline Planet B & 266.67 & 100 & 166.67 \\
\hline Sun B & 0 & 100 & -100 \\
\hline
\end{tabular}

Once a value is known in the column labeled " $\omega_{\text {gear/carrier }}$ " of Table 3.5 for any of the gears, the remaining values for this column can be calculated [27]. As long as the Carrier speed is known the calculations are quite simple. However, if the Carrier speed is not known, solving a system of equations may be required.

A second example is shown in Table 3.6, where Input A has an angular velocity of 100 RPM and Input B has an angular velocity of -100 RPM (i.e. Carrier speed not known). A series of seven equations with seven unknowns was used to arrive at a solution. Both examples in Tables 3.5 and 3.6 show how results from these calculations coincide to results using Equation (3.1), as values in the columns labeled $\omega_{\text {carrier }}$ are the averages of values of Inputs A and B (Sun Gears A and B).

Table 3.6: Another Example of using the Tabular Method

\begin{tabular}{|c|c|c|c|}
\hline Gear Name & $\omega_{\text {gear }}=$ & $\omega_{\text {carrier }}+$ & $\omega_{\text {gear } / \text { carrier }}$ \\
\hline Sun A & 100 & 0 & 100 \\
\hline Planet A & -166.67 & 0 & -166.67 \\
\hline Planet B & 166.67 & 0 & 166.67 \\
\hline Sun B & -100 & 0 & -100 \\
\hline
\end{tabular}

The second equation used in this analysis describes the torque relationship of the PD between the $3 \mathrm{I} / \mathrm{Os}$ and shall be referred to as the torque equation (3.4).

$$
T_{A}+T_{B}+T_{C}=0
$$


Torque of Input A plus torque of Input B plus torque of Output $\mathrm{C}$ is equal to zero. The torque seen at Inputs $\mathrm{A}, \mathrm{B}$ and Output $\mathrm{C}$ is represented by $T_{A}, T_{B}$ and $T_{C}$, respectively. As was noted earlier, this equation assumes zero efficiency loss.

The third equation used in this analysis describes the power relationship of the PD between the $3 \mathrm{I} / \mathrm{Os}$ and shall be referred to as the power equation (3.5).

$$
T_{A} * r p m A+T_{B} * r p m B+T_{C} * r p m C=0
$$

Power is equal to torque multiplied by angular velocity. If I/Os $\mathrm{A}, \mathrm{B}$ and $\mathrm{C}$ provide torque to the $\mathrm{PD}$ at a given angular velocity, the sum of their respective products would be equal to zero, again assuming no efficiency losses.

\subsection{Experimental Setup}

Figure 3.4 is a picture taken of the experimental setup showing the location and connection of each of the I/Os with the PD. To test the PD, a motor was rigidly connected to each of the I/Os, two via shafts and the other via sprockets and chain. In keeping with our labeling convention, the Prime Mover (PM) or main power source, is connected at Input A, the Control Motor (CM) at Input B and the Output Motor $(\mathrm{OM})$ at Output C. Between each of these connections is a continuousoutput torque sensor and a flexible coupling to compensate for any misalignment. The entire system was powered by both 110 and 220 Volt AC power with the use of several power supply units to convert from $\mathrm{AC}$ to $\mathrm{DC}$ power.

\subsubsection{Physical Layout}

To make this experimental setup a little easier to understand, a simplified image has been created, see Figure 3.5. The respective colors and location of each of the motors and torque sensors (a symbol for each of the torque sensors is also displayed), as seen in Figure 3.5, will also be an aid in displaying and interpreting experimental results, as each of the I/Os will not only be identified by the power source that is connected to it, but also by the color and symbol of the torque sensor at each location. 


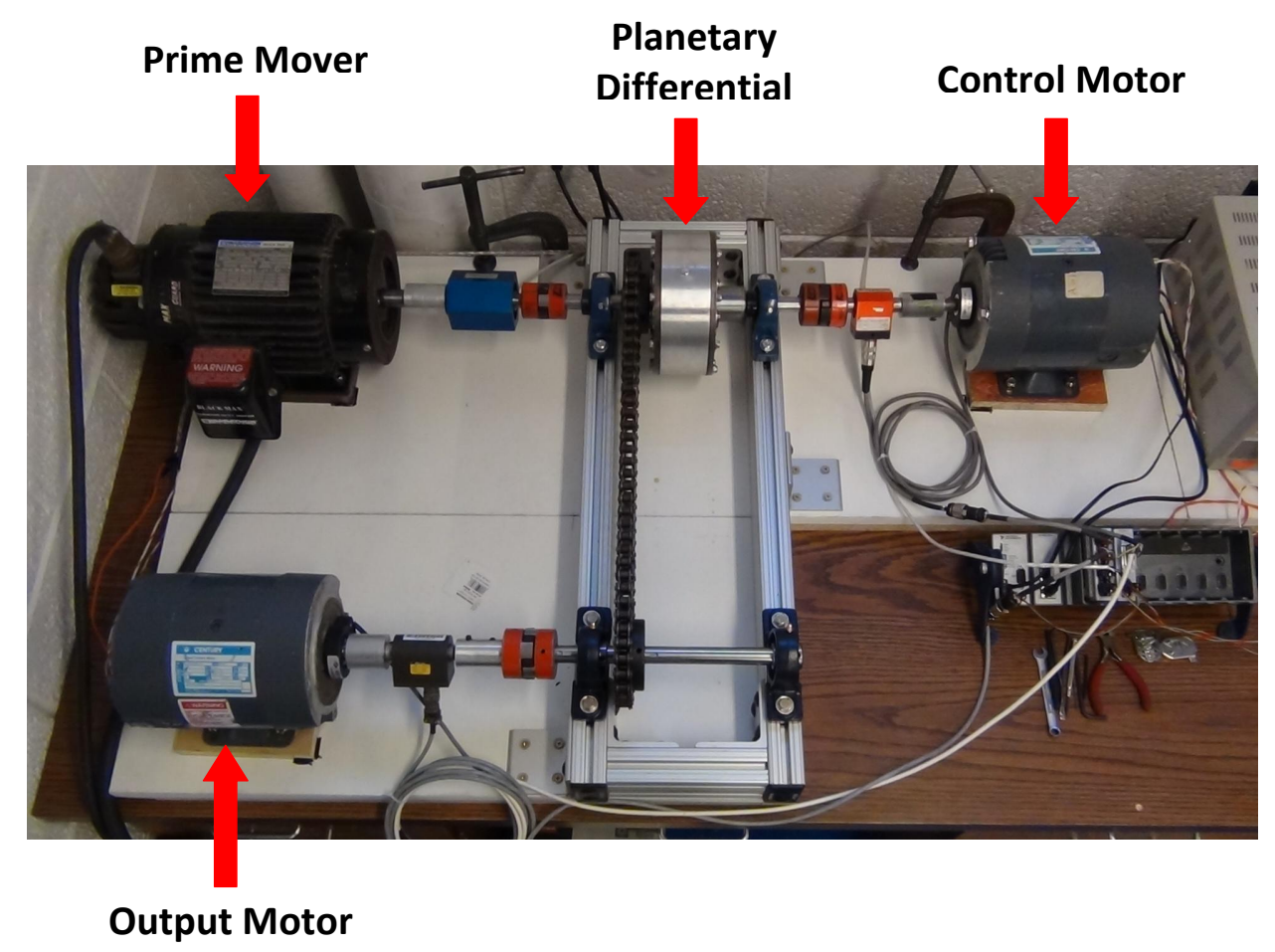

Figure 3.4: Experimental Setup for Testing of the PD

\subsubsection{Electric Motors}

Three electric motors were used in testing the PD, two provided input power and the third created (or simulated) a load. Later, in testing the PD, a mechanical (i.e. non-electronic) device was used to simulate a load. This device is discussed briefly in the section entitled "Output Motor". In all cases, torque and speed were measured at each of the I/Os throughout all tests to allow power in and out of the system to be measured.

\section{Prime Mover}

Only one type of Prime Mover (PM) was used in testing the PD. The PM, as seen in Figure 3.6, is a 2 horsepower, AC, 3-phase electric motor managed by a Danfoss VLT500 variable frequency drive controller. During all of its use under testing, it was held at various speeds, or brought up to speed, but always rotated in the same direction. Due to the high power of the PM, as compared to the other two electric motors, and the capability of its controller, it never varied in speed under load, but always maintained its intended angular velocity during testing. 


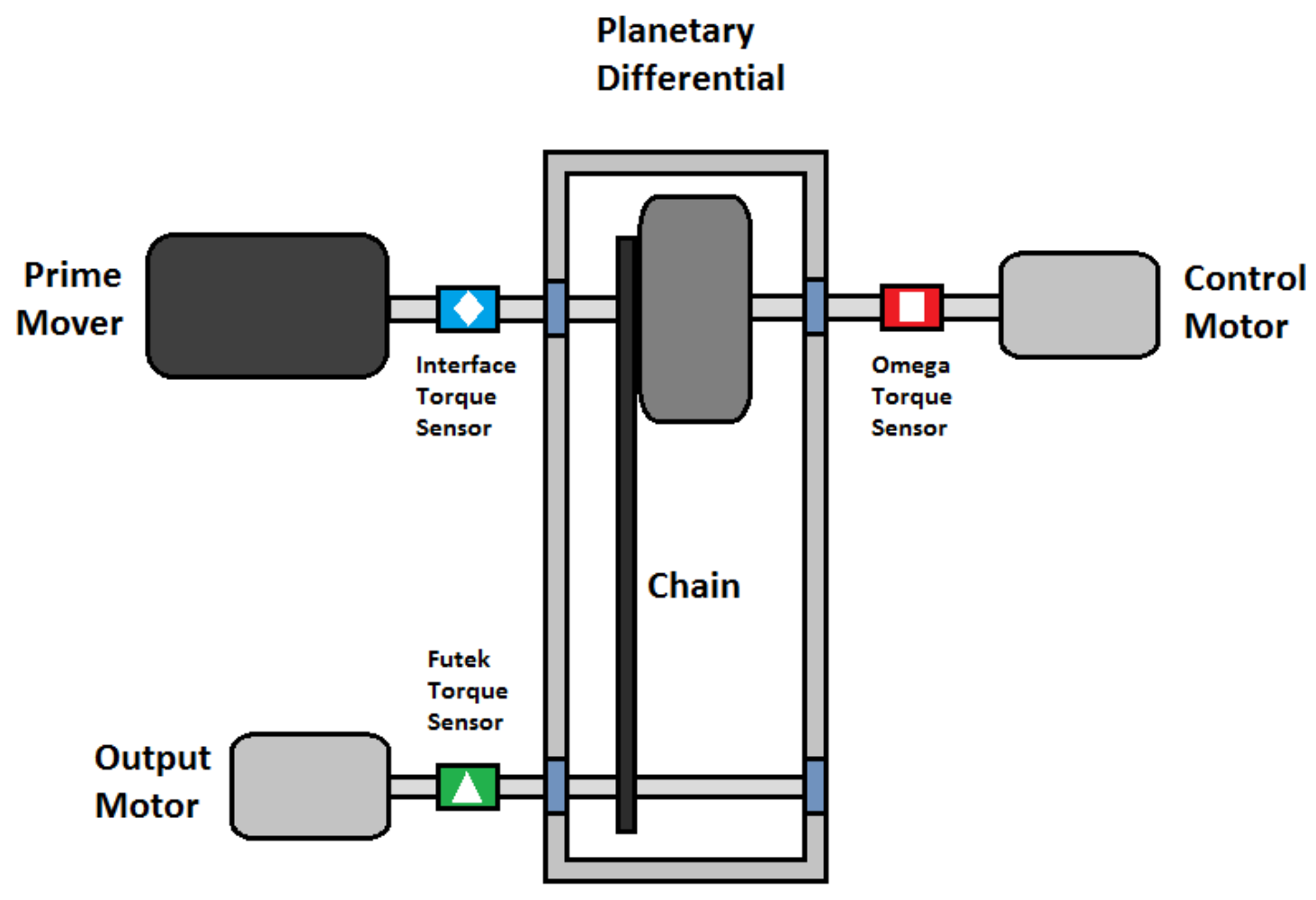

Figure 3.5: Simplified view of Experimental Setup

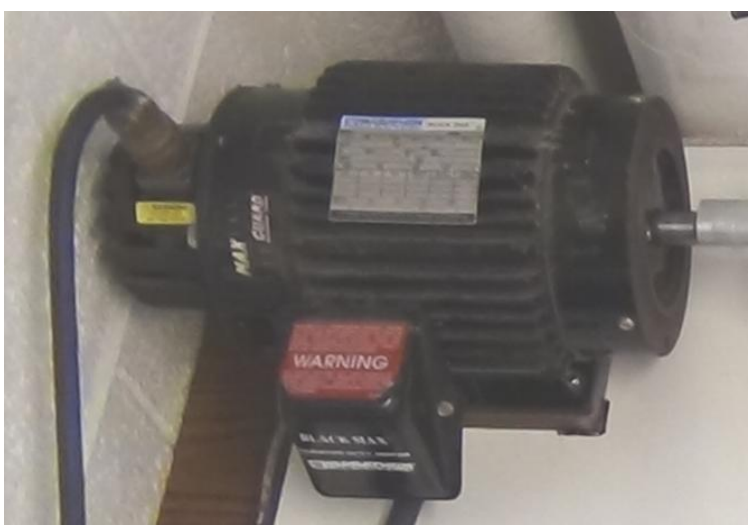

Figure 3.6: AC Motor used as Prime Mover

\section{Control Motor}

Only one type of Control Motor (CM) was used in testing the PD. The CM, as seen in Figure 3.7, is a $\frac{1}{4}$ horsepower, DC, shunt-wound electric motor. See Table 3.7 for more details about this motor. It was operated at varying speeds in varying directions, and is given the label of 
Control Motor because it is was used to control the speed of the output, thus providing the step-less ratio change characteristics of a CVT.

Table 3.7: Specifications of DC Motors

\begin{tabular}{|c|c|}
\hline Parameter & Specification \\
\hline Manufacturer & Century Electric \\
\hline Arm Voltage & $90 \mathrm{~V}$ \\
\hline Arm Current & $2.69 \mathrm{~A}$ \\
\hline Field Voltage & $100 / 50$ \\
\hline Field Current & $.32 / .64 \mathrm{~A}$ \\
\hline Power & $1 / 4 \mathrm{HP}$ \\
\hline Max RPM & 1750 \\
\hline Part & $6-217375-01$ \\
\hline Frame & $\mathrm{B} 56 \mathrm{C}$ \\
\hline
\end{tabular}

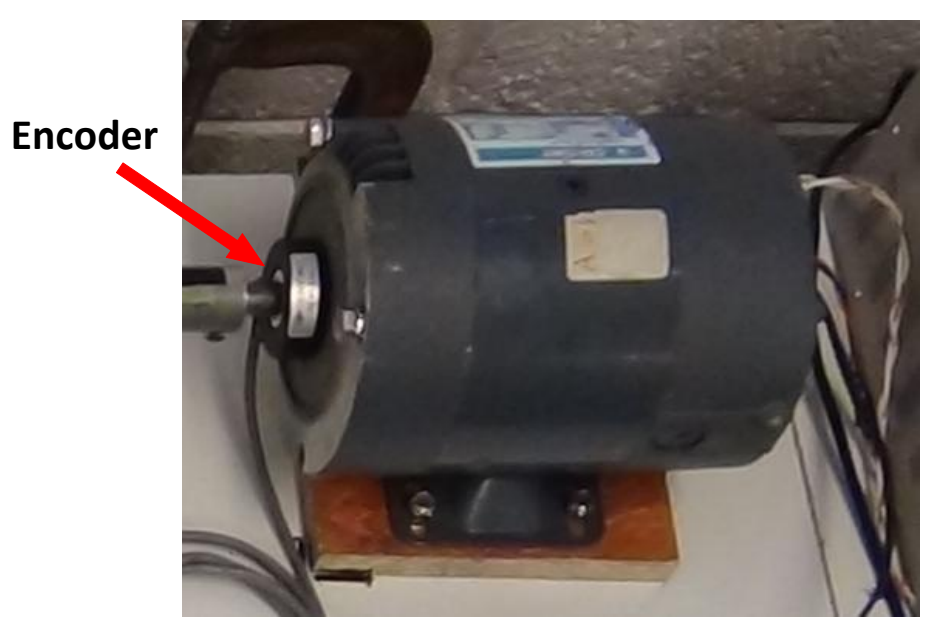

Figure 3.7: DC Motor used as Control Motor

Also seen in Figure 3.7, as indicated by the red arrow, is the encoder used on this motor to measure angular velocity. A similar encoder is found on the Output Motor, as well. For more details about this encoder see Table 3.8 . 
Table 3.8: Specifications of Encoders

\begin{tabular}{|c|c|}
\hline Parameter & Specification \\
\hline Pulses per Revolution & 1000 \\
\hline Type & Optical Quadrature \\
\hline Supply Voltage & $+5 \mathrm{~V}$ \\
\hline Frequency Response & $100 \mathrm{kHz}$ \\
\hline Output Type & Voltage \\
\hline
\end{tabular}

\section{Output Motor}

Only one type of Output Motor (OM) was used in testing the PD. The OM (same type of motor as the CM), as seen in Figure 3.8, is also a $\frac{1}{4}$ horsepower, DC, shunt-wound electric motor. The OM was operated at several different, but constant loads. The OM simulated a load to the PD by supplying it with an initial voltage and current from a DC power supply.

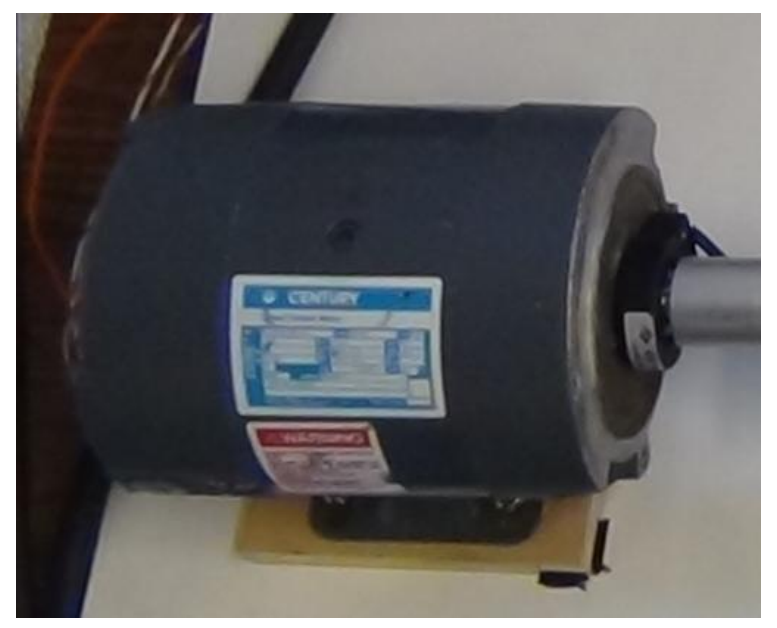

Figure 3.8: DC Motor used as Output Motor

As was mentioned earlier, a load was also simulated at this location by the use of a mechanical brake. An image of the braking device can be seen in Figure 3.9. This device was built in-house using rubber pads fastened to aluminum brackets that could be tightened to the appropriate torque load. The accuracy of this load was calibrated each time by using the local torque sensor read-out at this location. 


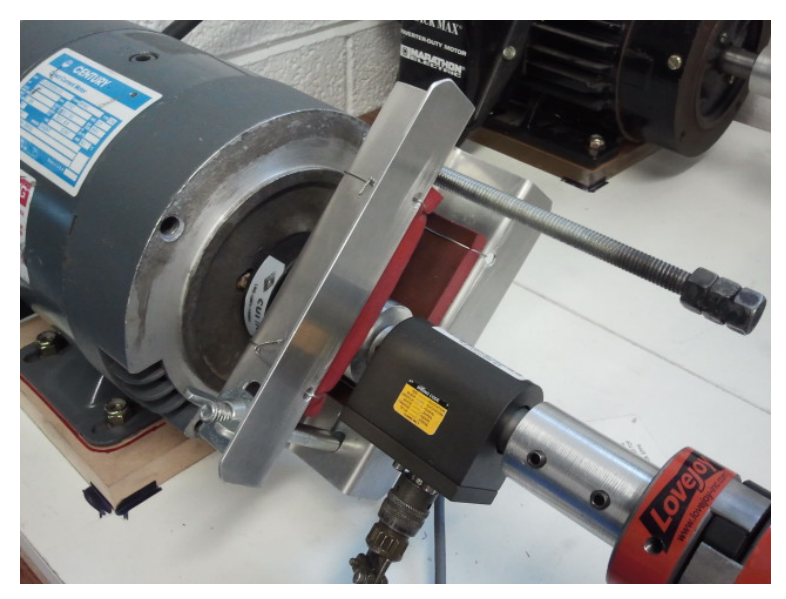

Figure 3.9: Mechanical Braking Device used to Simulate Loading

\subsubsection{Data Collection}

During all of the testing, data was collected using a few different sensors and encoders and input into a computer through use of a data acquisition system.

\section{Torque Sensors}

To collect torque measuremens, three different torque sensors were used to continually read data. A picture of each of these is shown in Figure 3.10. The location of each of these toque sensors in the experimental setup is easily noted not only by the name of the sensor, but also by its color (See Figure 3.5). Table 3.9 provides locational information for each of these sensors. Table 3.10 provides additional information for each of these sensors.

Table 3.9: Torque Sensor Locational Information

\begin{tabular}{|c|c|c|}
\hline Sensor Brand & Color & Location \\
\hline Interface & Blue & Prime Mover \\
\hline Omega & Red & Control Motor \\
\hline Futek & Green & Output Motor \\
\hline
\end{tabular}




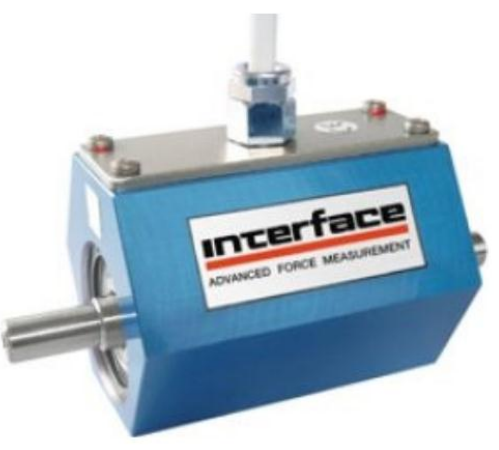

(a) Interface

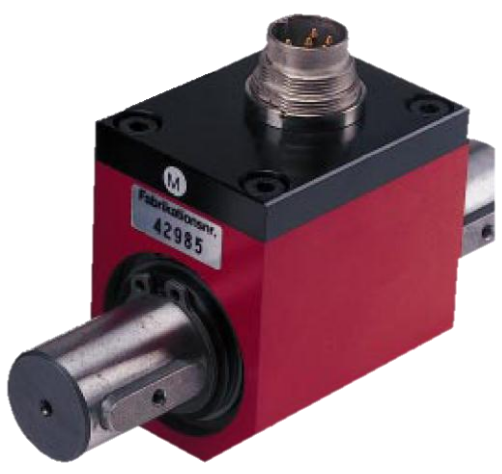

(b) Omega

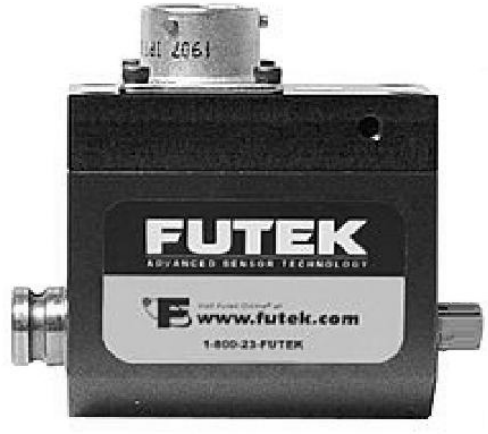

(c) Futek

Figure 3.10: Torque Sensors used at each of the 3 I/Os

Table 3.10: Additional Torque Sensor Information

\begin{tabular}{|c|c|c|c|}
\hline Parameter & Interface & Omega & Futek \\
\hline Type & Slip Ring & Slip Ring & Slip Ring \\
\hline Measurement Method & Angular Deflection & Angular Deflection & Angular Deflection \\
\hline Excitation Voltage & $+15 \mathrm{~V}$ & $+5 \mathrm{~V}$ & $+5 \mathrm{~V}$ \\
\hline Calibration & $\mathrm{n} / \mathrm{a}$ & $2 \mathrm{mV} / \mathrm{V}_{\text {exc }}$ & $1.5 \mathrm{mV} / \mathrm{V}_{\text {exc }}$ \\
\hline Max Torque & $50 \mathrm{Nm}$ & $20 \mathrm{Nm}$ & $200 \mathrm{in} * \mathrm{lb}$ \\
\hline
\end{tabular}

\section{Encoders}

As can be seen in Figure 3.7, encoders were used at both the CM and OM to measure angular velocity. The PM speed, because it remained constant during all testing, was recorded manually. Again, additional information for these encoders is contained in Table 3.8.

\section{Data Acquisition}

To acquire measurements from each piece of hardware, a cRIO with plug-in modules was used, as seen in Figure 3.11. This cRIO, along with the modules, were manufactured by National Instruments. The cRIO collected measured data from all three torque sensors, both encoders, along with voltage and/or current read from one of the power supplies used to power the DC motors. The cRIO also provided power to both encoders and both the Omega and Futek torque sensors. A separate power supply was used to power the Interface torque sensor. 


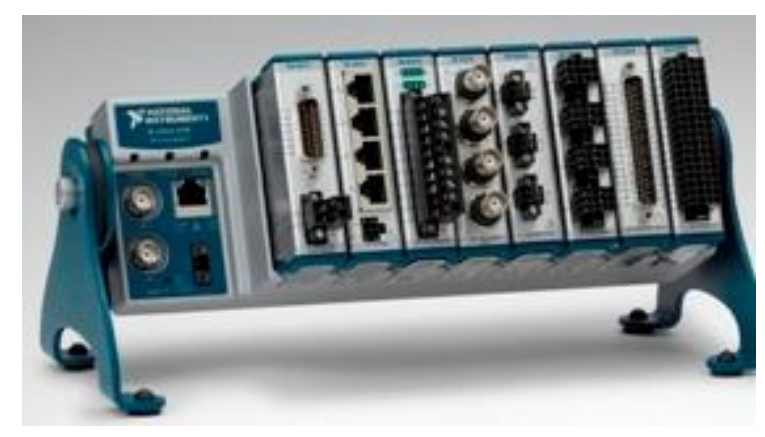

Figure 3.11: Data Aquisition

\section{LabView Program}

The computer software used to analyze the imported data was a project file from Labview, also by National Instruments. A screen shot of this layout can be seen in Figure 3.12. The data was imported to Labview and then written to and saved in a separate file.

\section{Excel}

The measured/collected data from the cRIO and Labview Program was saved and analyzed in a separate file using Microsoft Excel. Excel was also used to generate all plots/graphs displayed in this research. 


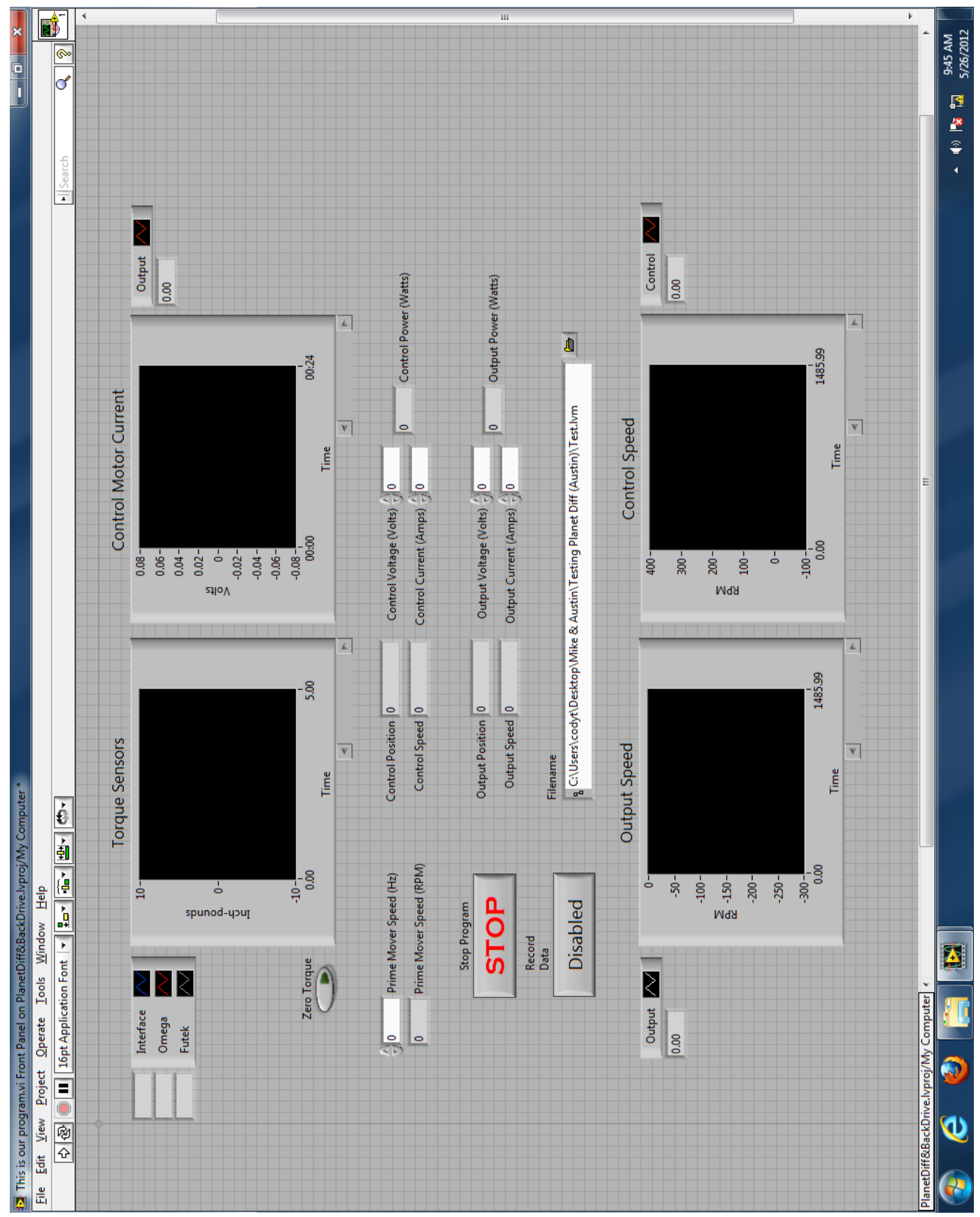

Figure 3.12: Labview Program used for Testing the PD 


\section{CHAPTER 4. RESULTS}

\subsection{Repeat of Previous Testing}

To ensure that the Planetary Differential could be appropriately compared to the GleasonTorsen Differential, all former tests performed on the GTD were also performed on the PD. Although the $\mathrm{x}$-axis label is different between the two sets of tests, the data can still be compared

directly, as both sets of data have the same y-axis and obey Equations (2.3), (2.10) and (2.4), repeated here for convenience as Equations (4.1), (4.2) and (4.3), respectively; where Equations (4.2) and (4.3) are, again, assuming zero loss of efficiency.

$$
\begin{gathered}
r p m C=\frac{r p m A+r p m B}{2} \\
T_{A}+T_{B}+T_{C}=0 \\
T_{A} * r p m A+T_{B} * r p m B+T_{C} * r p m C=0
\end{gathered}
$$

\subsubsection{RPM Measurements}

This section demonstrates that the PD obeys Equation (4.1). Figures 4.1 through 4.5 show the angular velocity data measured (in revolutions per minute) for both the PD and GTD for Tests 1s, 2s, 1o, 2o and Crossover, respectively. The colors and symbols (diamonds, squares and triangles) of each of the sets of data points are displayed in a similar manner enabling the reader to easily compare the data from both I/O systems. In each of the sets of compared data for these figures, "rpmA" for the GTD is synonymous with "PM Speed" for the PD and so on, according to color and symbol. 


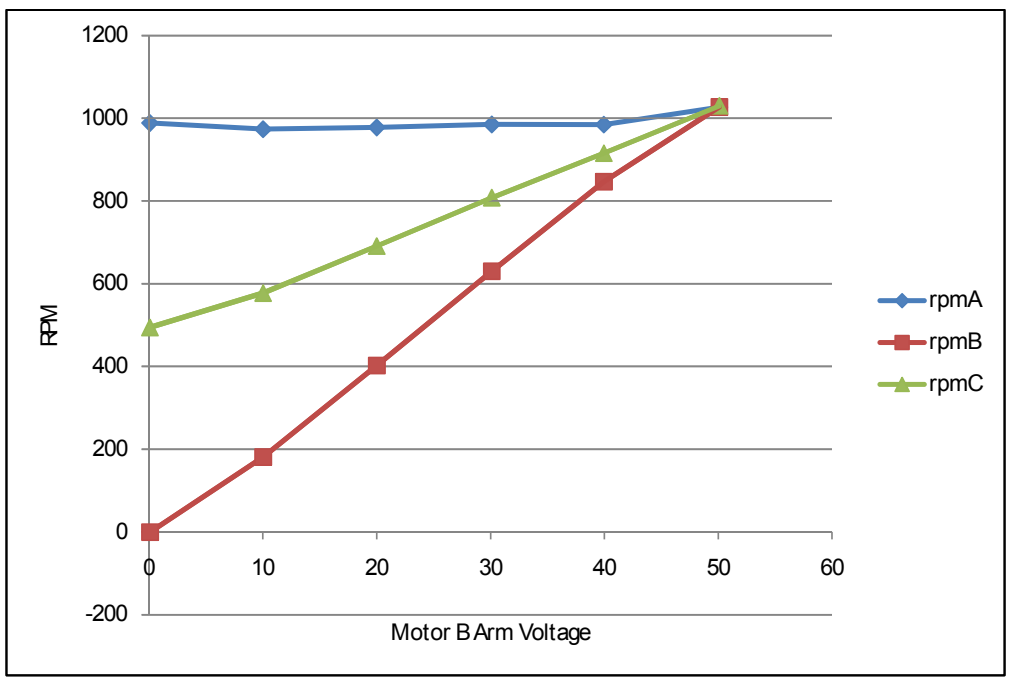

(a) GTD

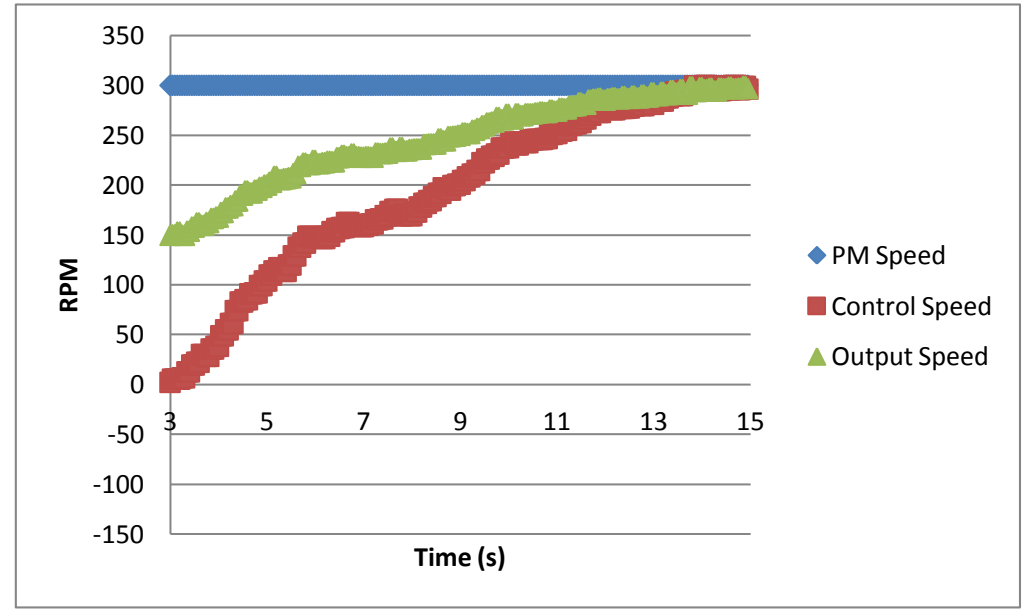

(b) PD

Figure 4.1: Test 1s - Comparison of Anglular Velocity

It can be seen from these plots that the PD obeys Equation (4.1). For example, in Figure 4.1 the PM speed (Input A) remains constant, while Control speed (Input B) increases. During this process the Output speed (Output C) remains exactly between the two inputs. This shows that the Output speed is the average of the two Input speeds, A and B. 


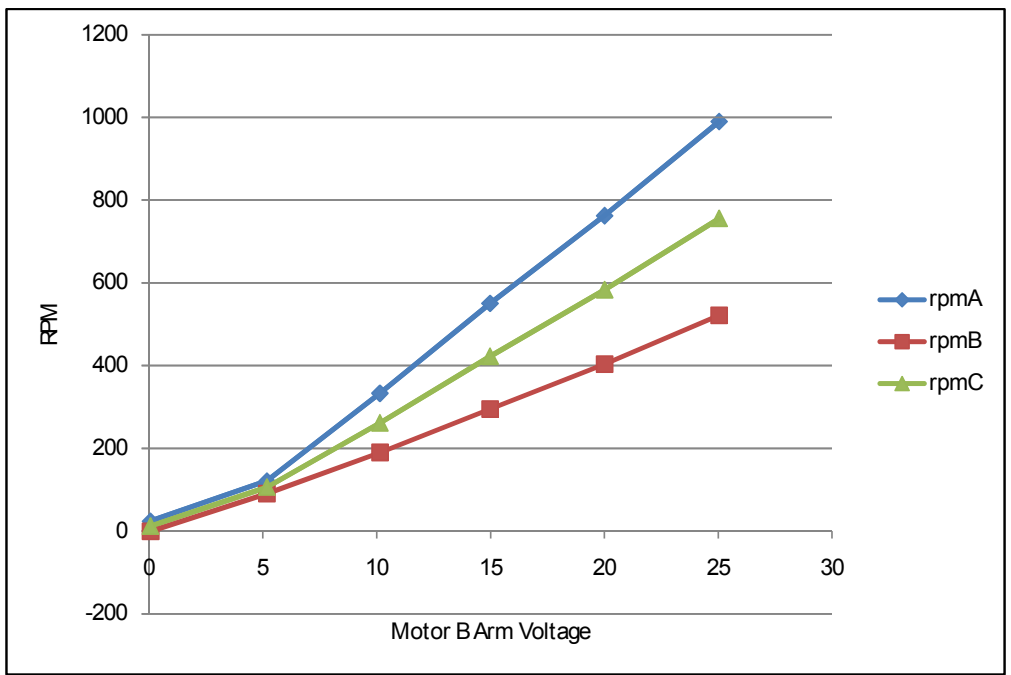

(a) GTD

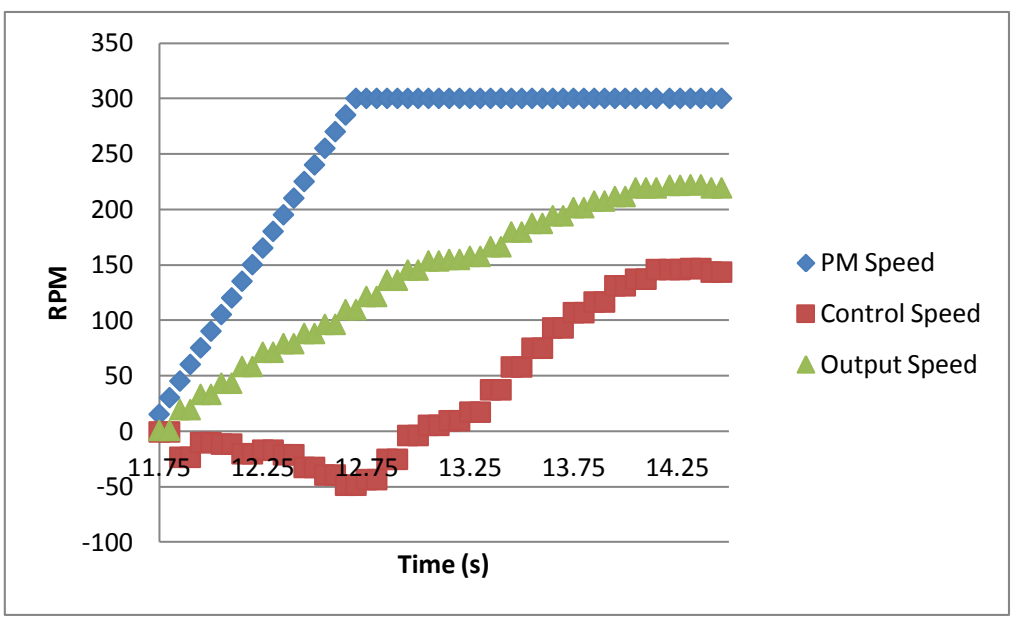

(b) PD

Figure 4.2: Test 2s - Comparison of Anglular Velocity 


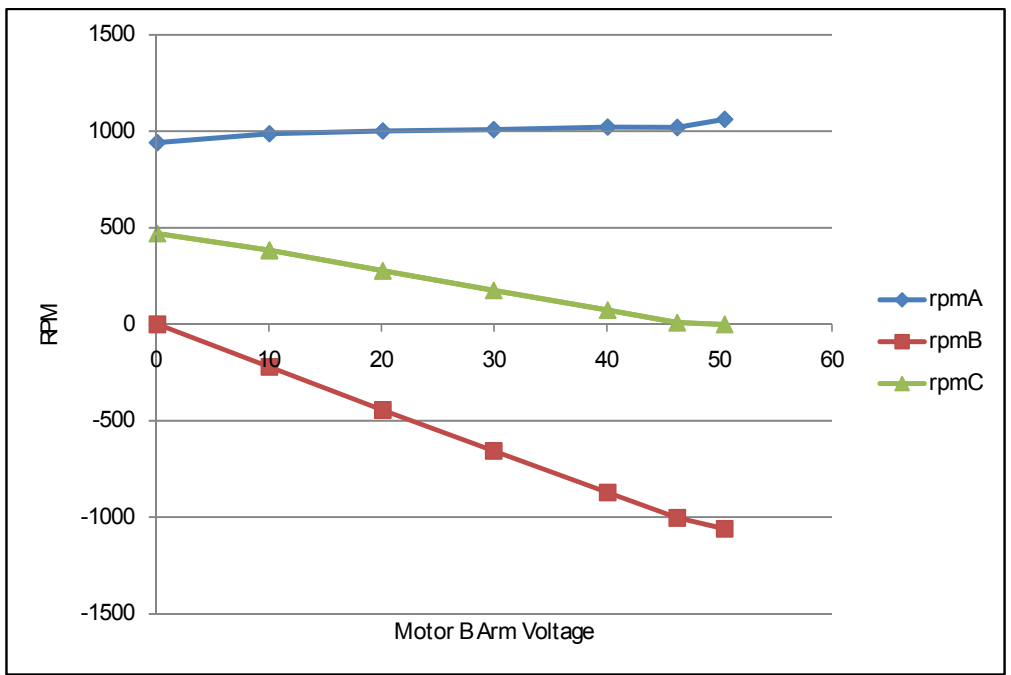

(a) GTD

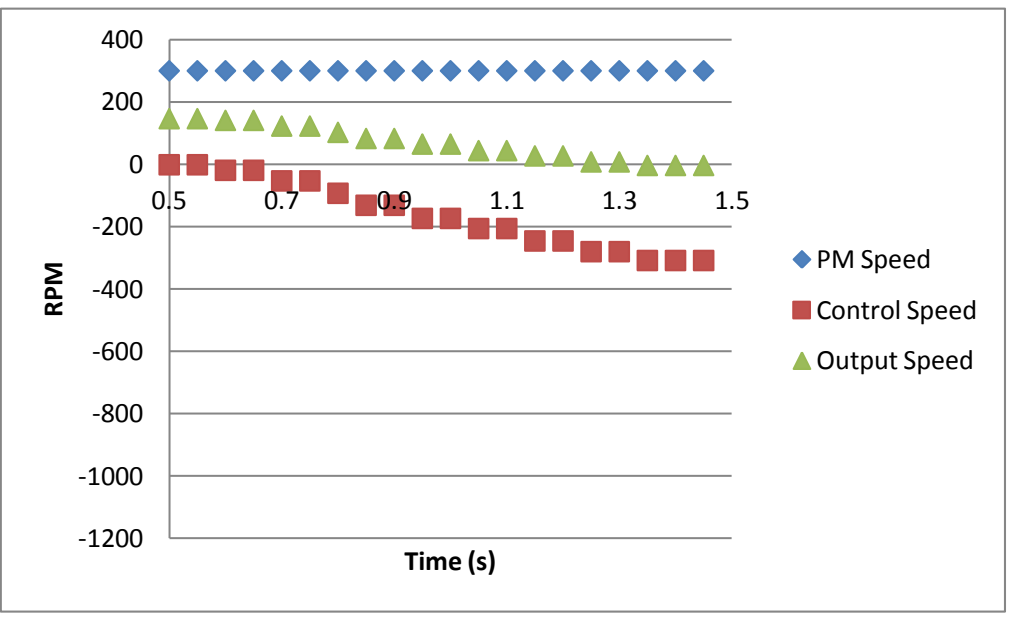

(b) $\mathrm{PD}$

Figure 4.3: Test 1o - Comparison of Anglular Velocity 


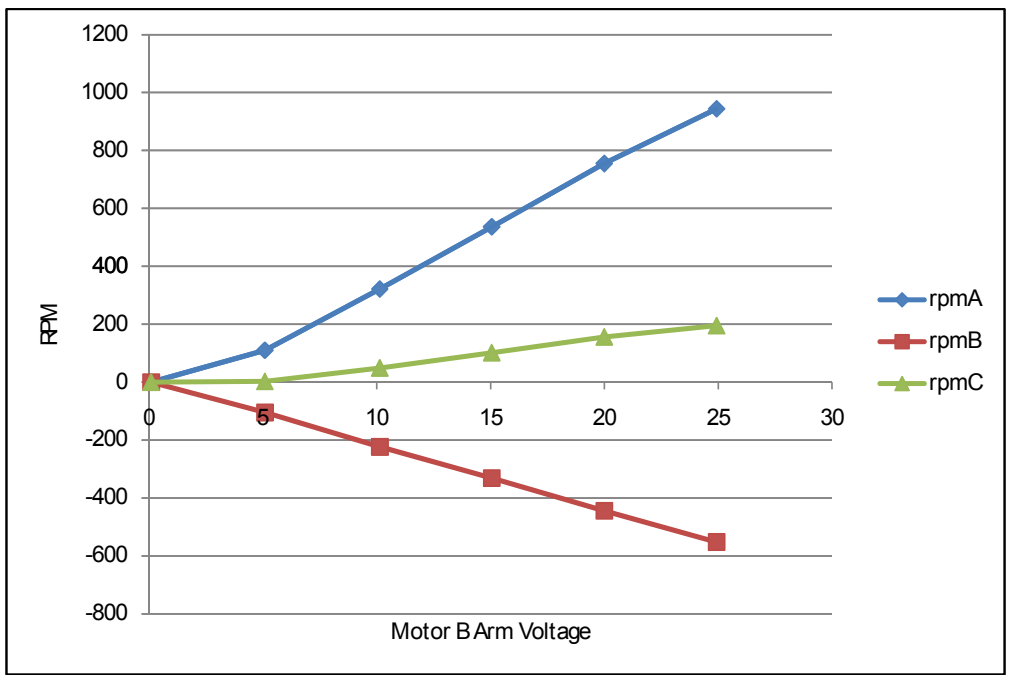

(a) GTD

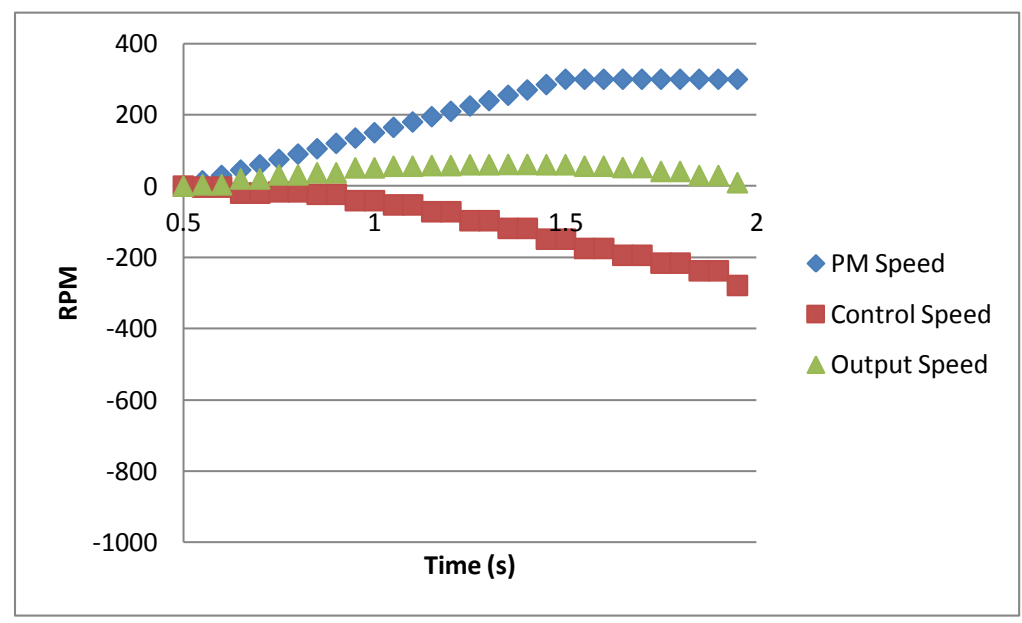

(b) PD

Figure 4.4: Test 2o - Comparison of Anglular Velocity 


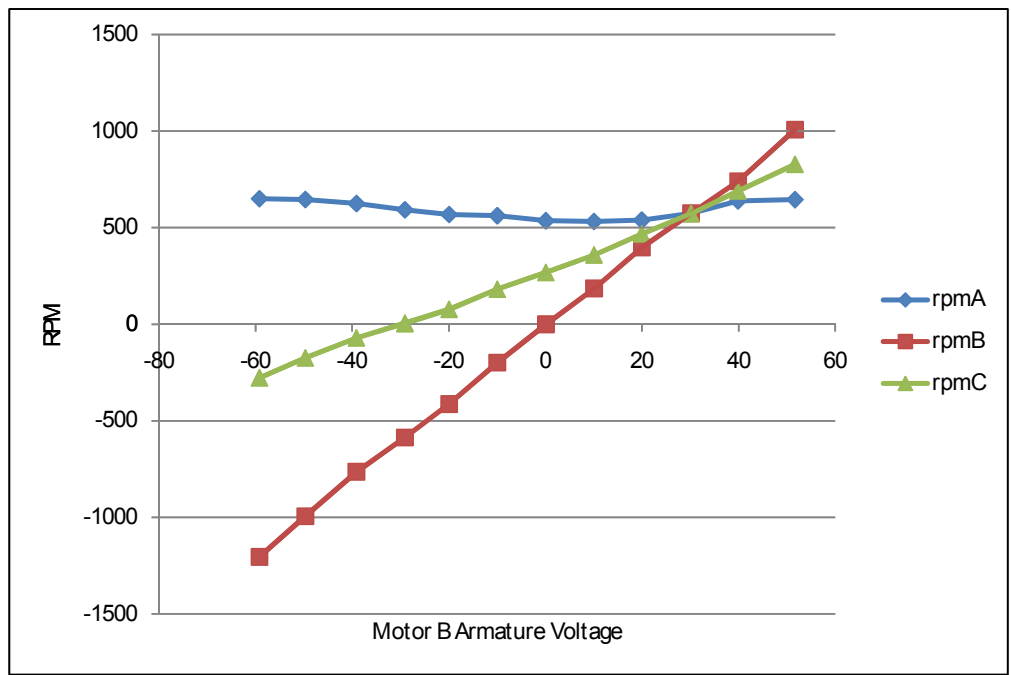

(a) GTD

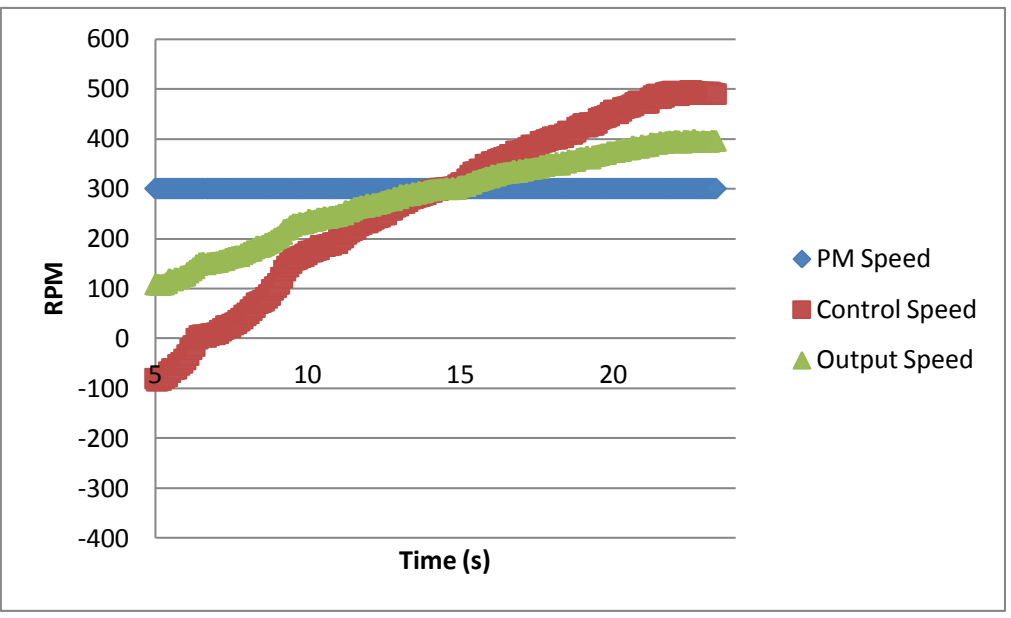

(b) PD

Figure 4.5: Test Crossover - Comparison of Anglular Velocity 


\subsubsection{Torque Measurements}

This section demonstrates that the PD obeys Equation (4.2). Figures 4.6 through 4.10 show the torque data measured (in inch pounds) for both the PD and GTD for Tests 1s, 2s, 1o, 2o and Crossover, respectively. The colors of each of the sets of data points are, again, displayed in a similar manner enabling the reader to easily compare the data from both I/O systems. In each of the sets of compared data for these figures, "Ta" for the GTD is synonymous with "Interface Torque" for the PD and so on, again, according to color and symbol.

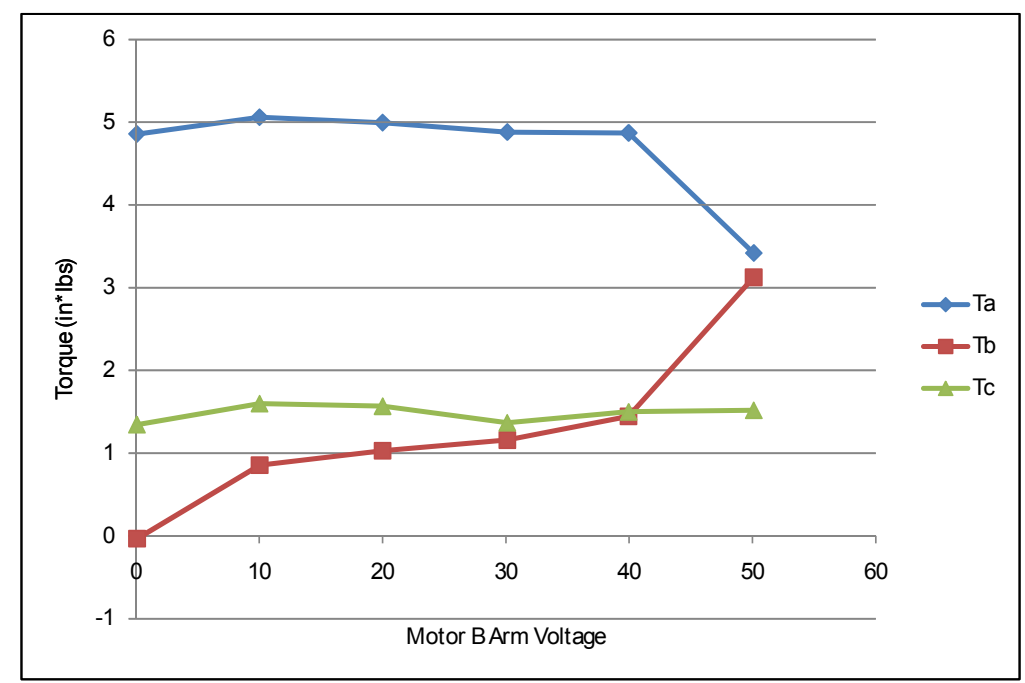

(a) GTD

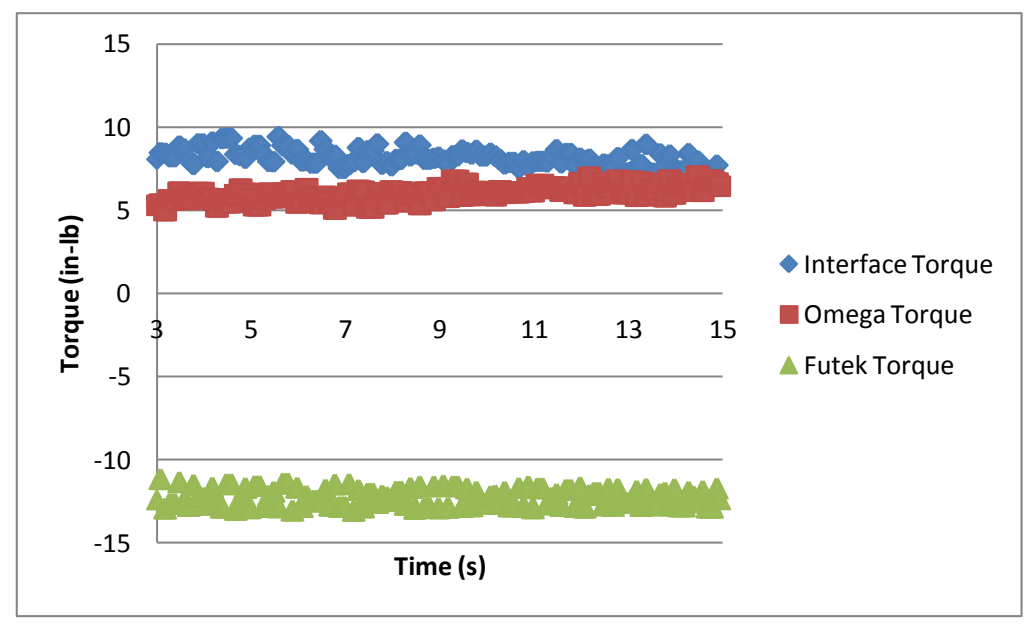

(b) PD

Figure 4.6: Test 1s - Comparison of Torque 


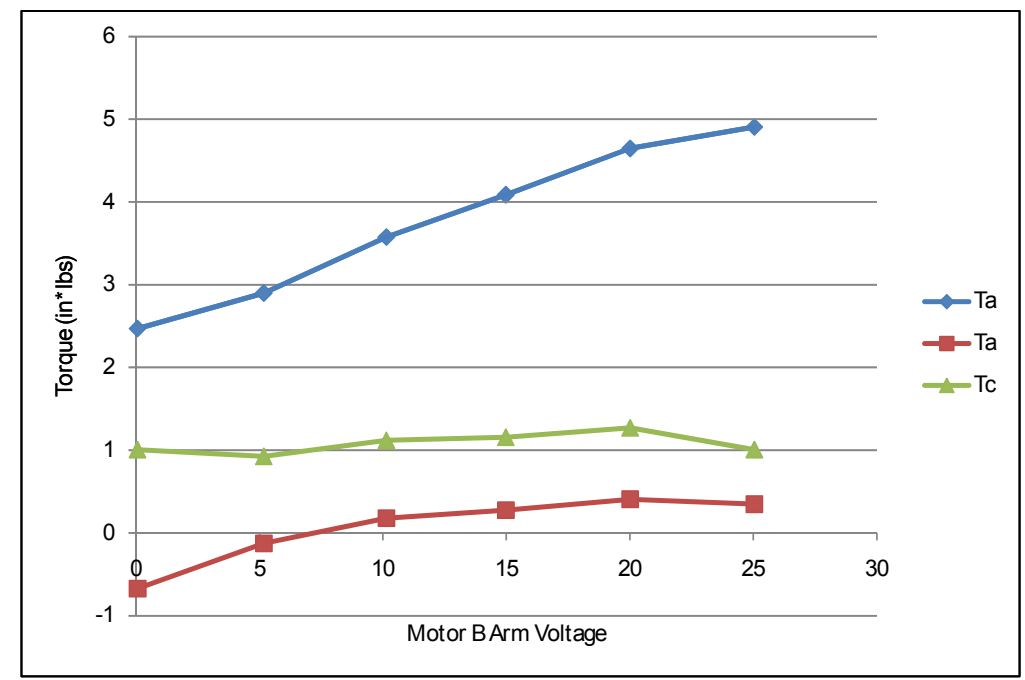

(a) GTD

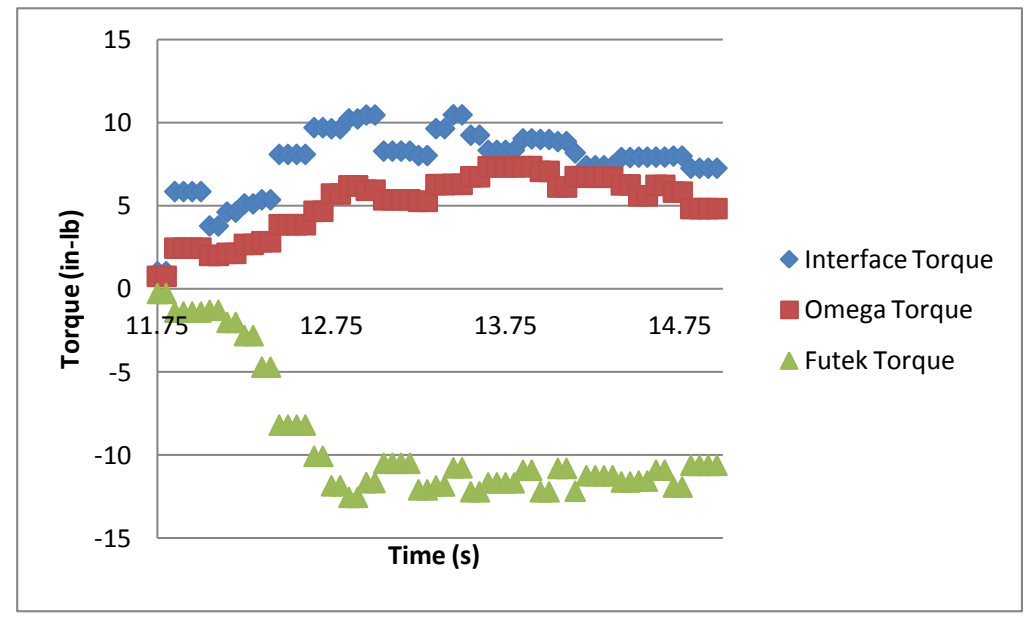

(b) PD

Figure 4.7: Test 2s - Comparison of Torque

The torque data from this section shows how much torque was present at each of the I/Os during each of the tests, for both the GTD and PD. It should be noted that this data will inherently be and appear different due to various factors including: applied load, sample rate (or method) and system efficiency. As the applied load varies the torque will also vary. If the sample rate is faster (which was the case with the PD data) the point-to-point location of data will not be as smooth. The biggest factor in the difference between torque values would be contributed to the system efficiency. Efficiency losses of the system, meaning the GTD or PD, have a direct affect on the torque seen at each of that system's I/Os. Angular velocity will always remain unaffected 


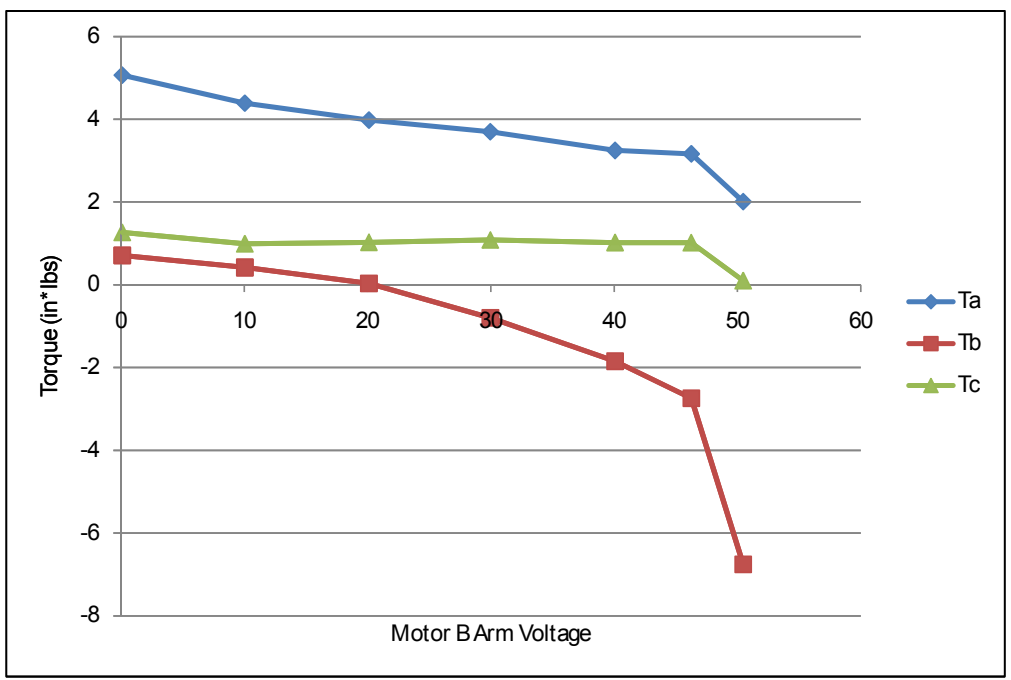

(a) GTD

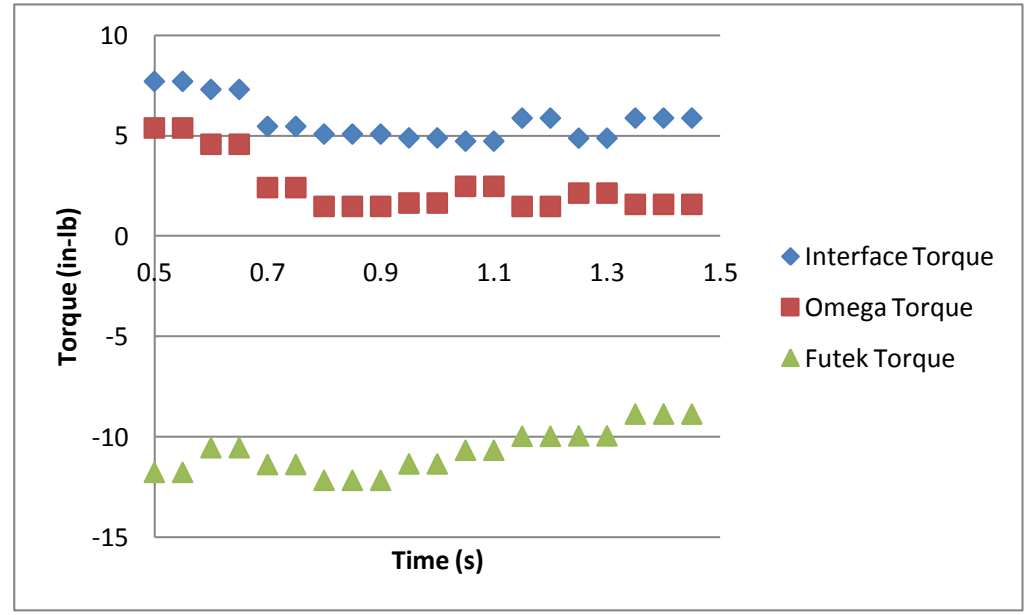

(b) PD

Figure 4.8: Test 1o - Comparison of Torque

by efficiency loss, as shown in Equation (4.1), but torque will not. This fact introduces another variable in the power equation (4.3) as shown now in Equation (4.4)

$$
T_{A} * r p m A+T_{B} * r p m B+T_{C} * r p m C-P_{l o s s}=0
$$

where $P_{\text {loss }}$ represents the loss of power due to the inefficiency of a given system. 


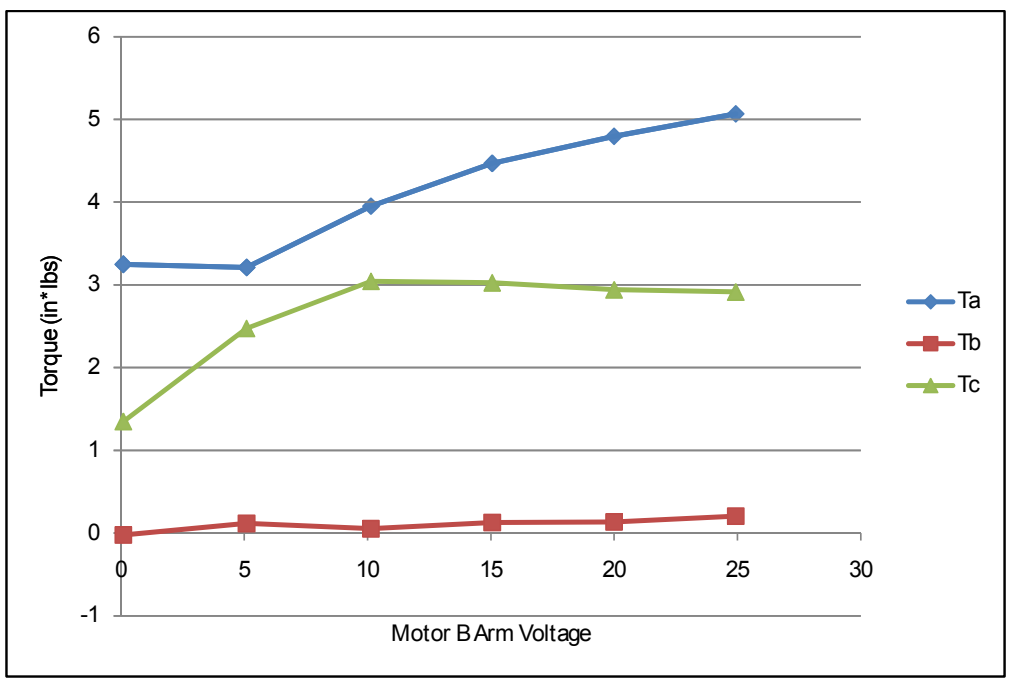

(a) GTD

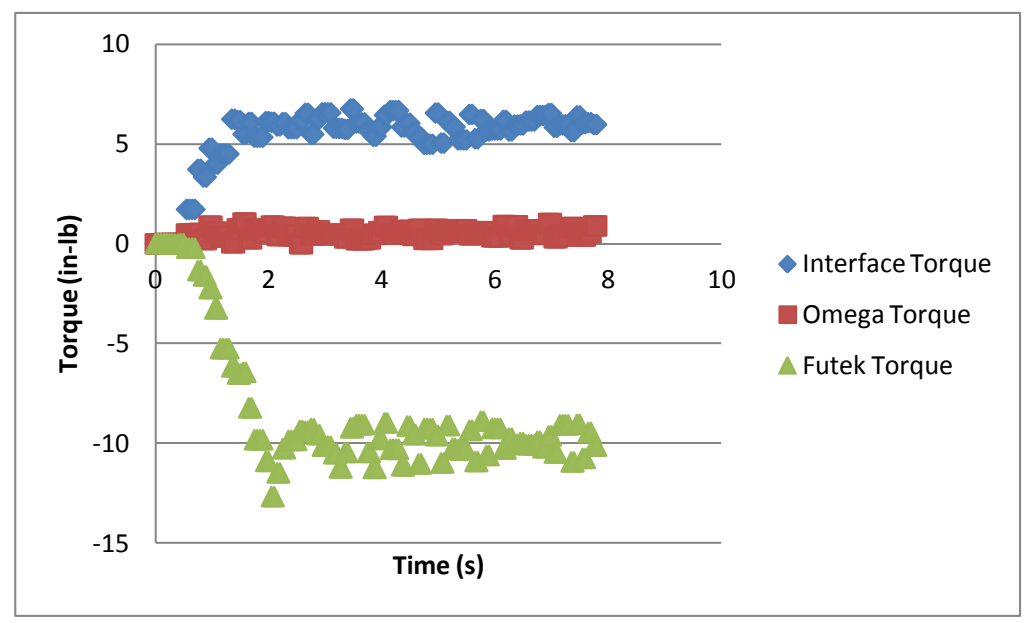

(b) PD

Figure 4.9: Test 2o - Comparison of Torque 


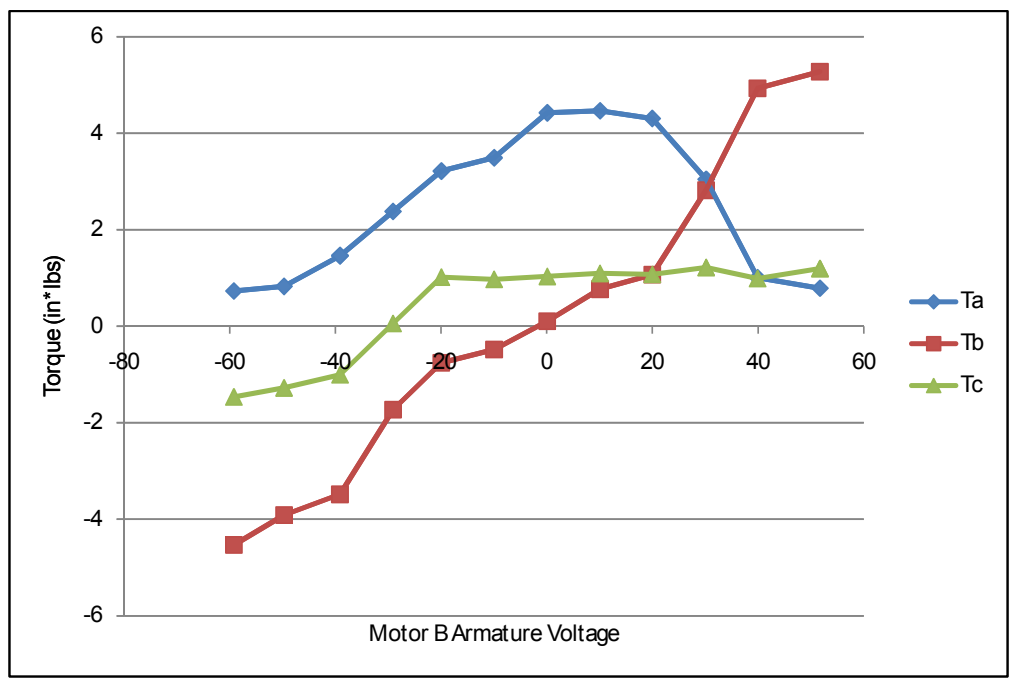

(a) GTD

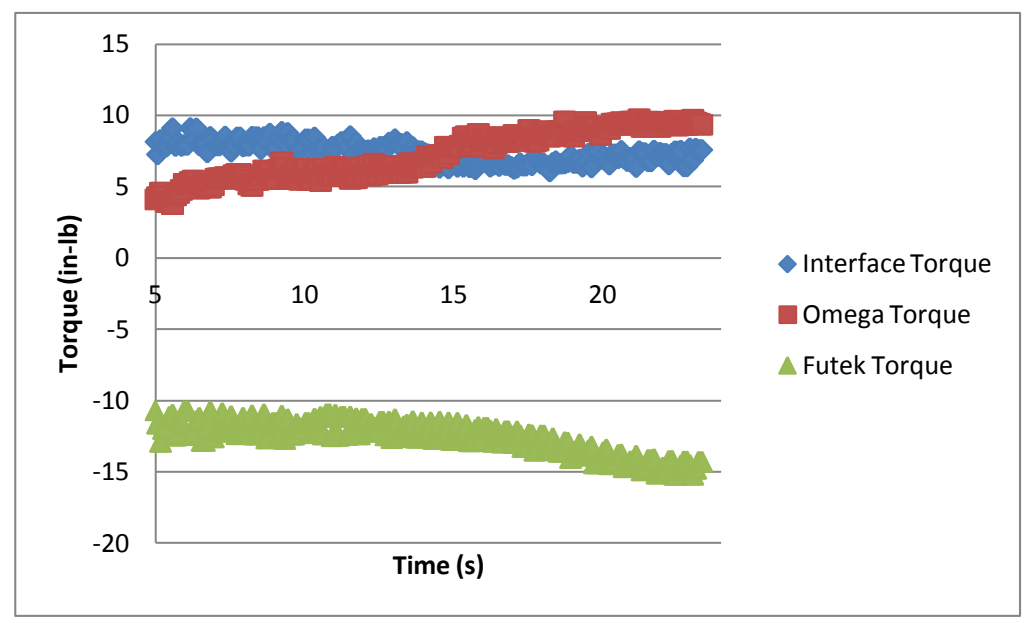

(b) PD

Figure 4.10: Test Crossover - Comparison of Torque 


\subsubsection{Power Measurements}

By using the angular velocity and torque data in the Figures 4.1 through 4.10, power in, power out and power loss of the system can be calculated. Figures 4.11 through 4.15 show the power data displayed in horsepower for both the PD and GTD for Tests 1s, 2s, 1o, 2o and Crossover, respectively. Power data for these figures was calculated as before using Equation (2.1).

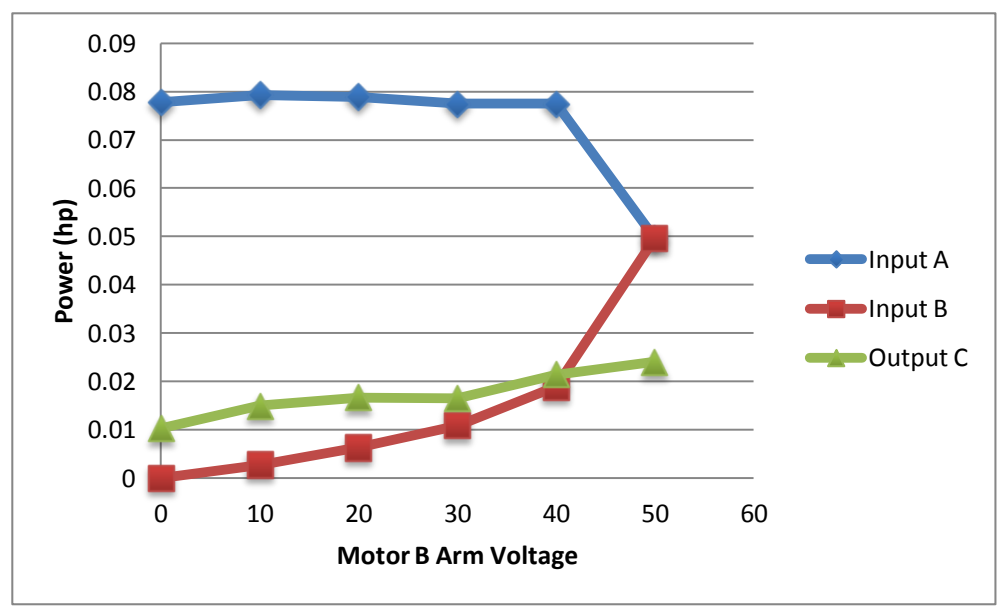

(a) GTD

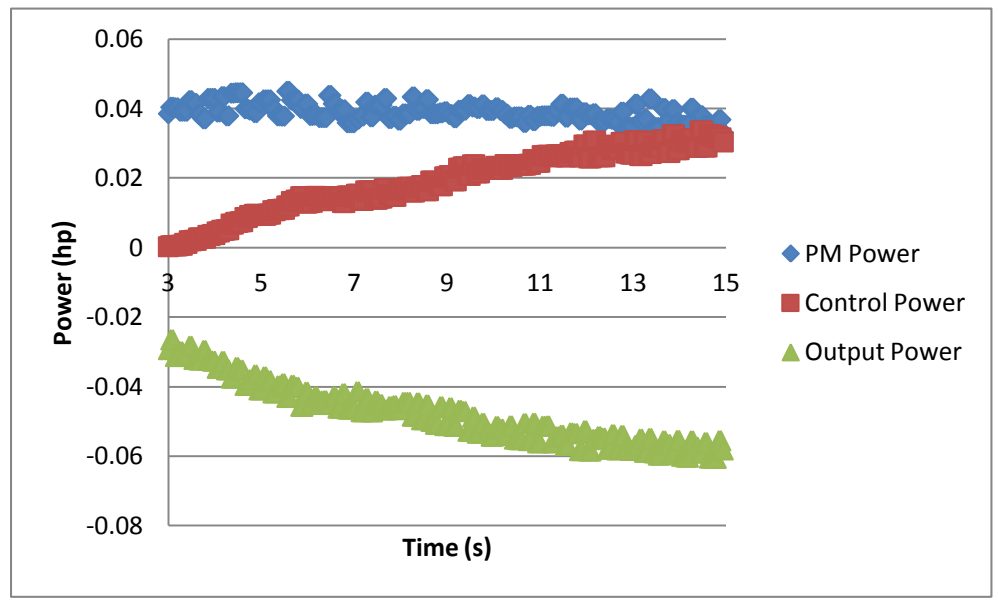

(b) PD

Figure 4.11: Test 1s - Comparison of Power 


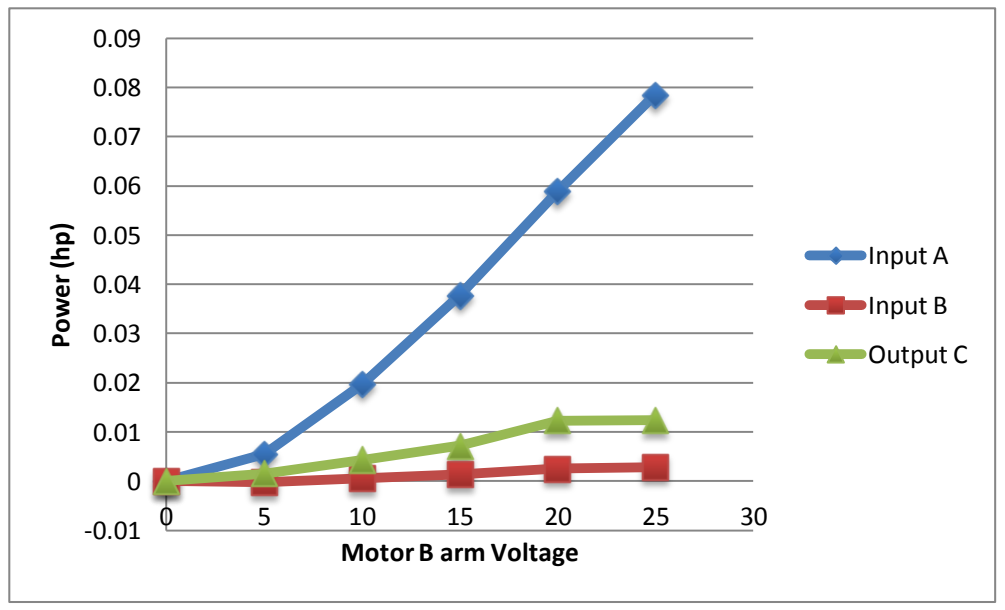

(a) GTD

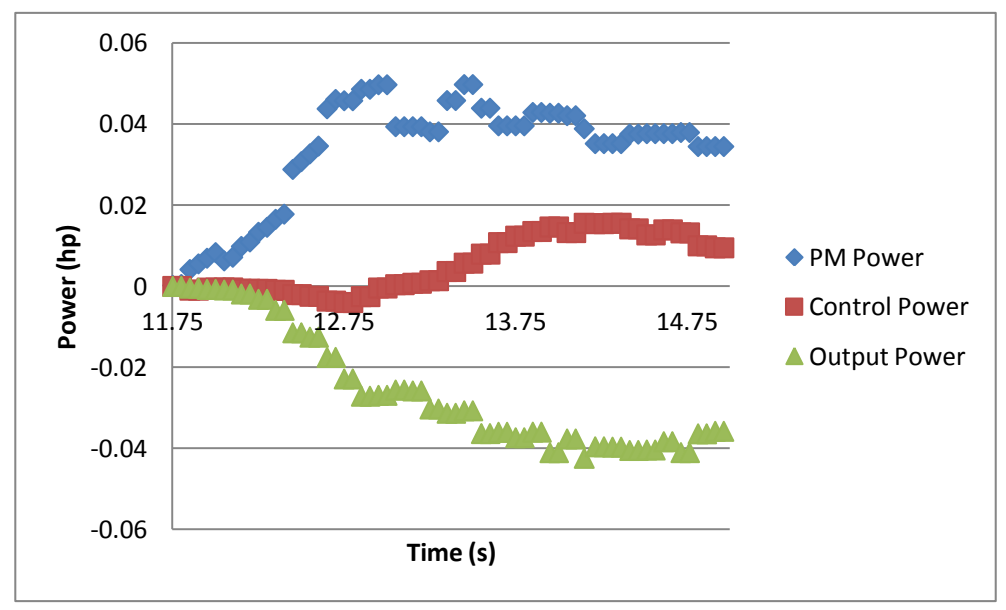

(b) PD

Figure 4.12: Test 2s - Comparison of Power 


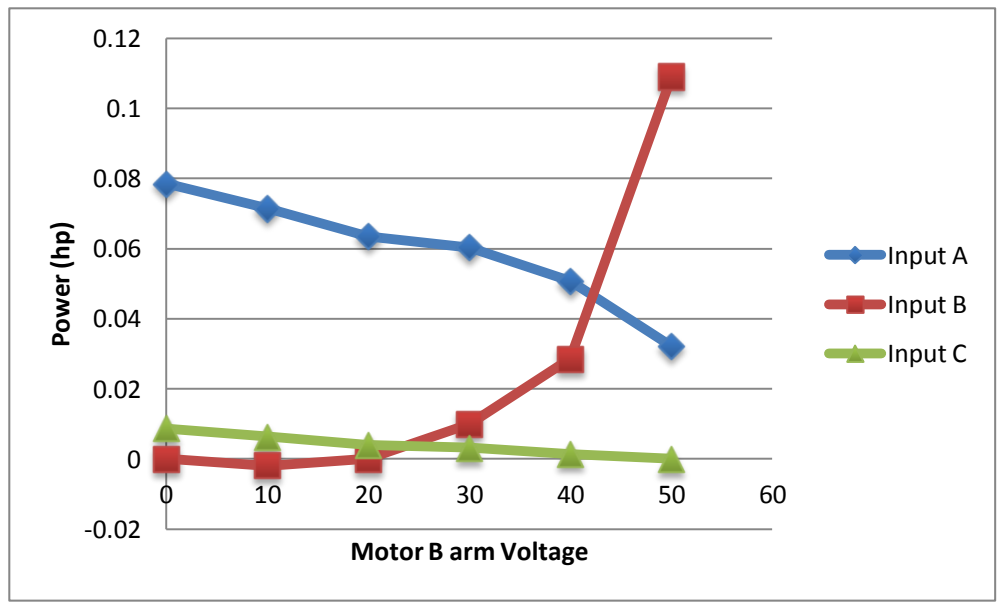

(a) GTD

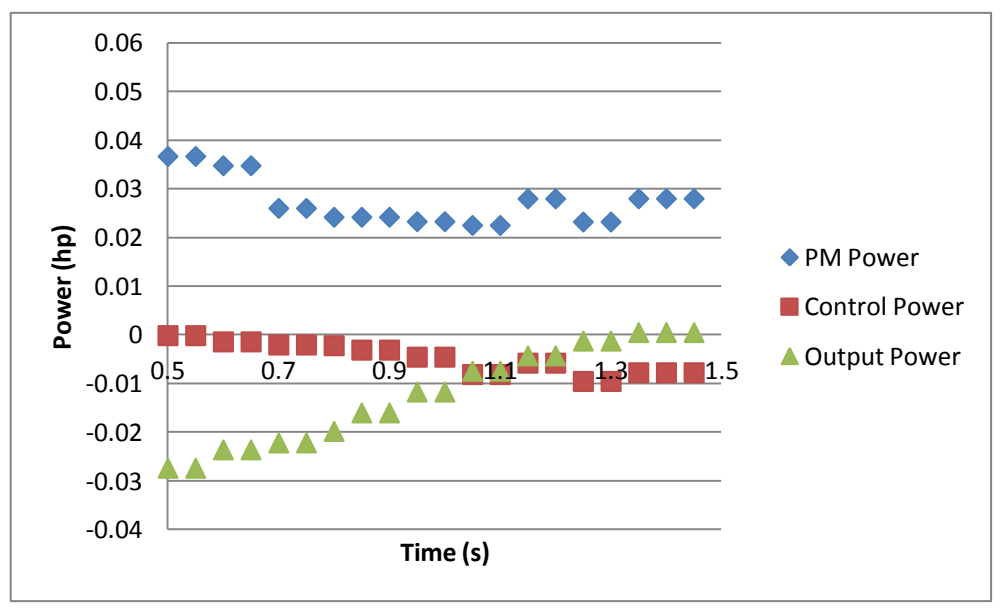

(b) PD

Figure 4.13: Test 1o - Comparison of Power 


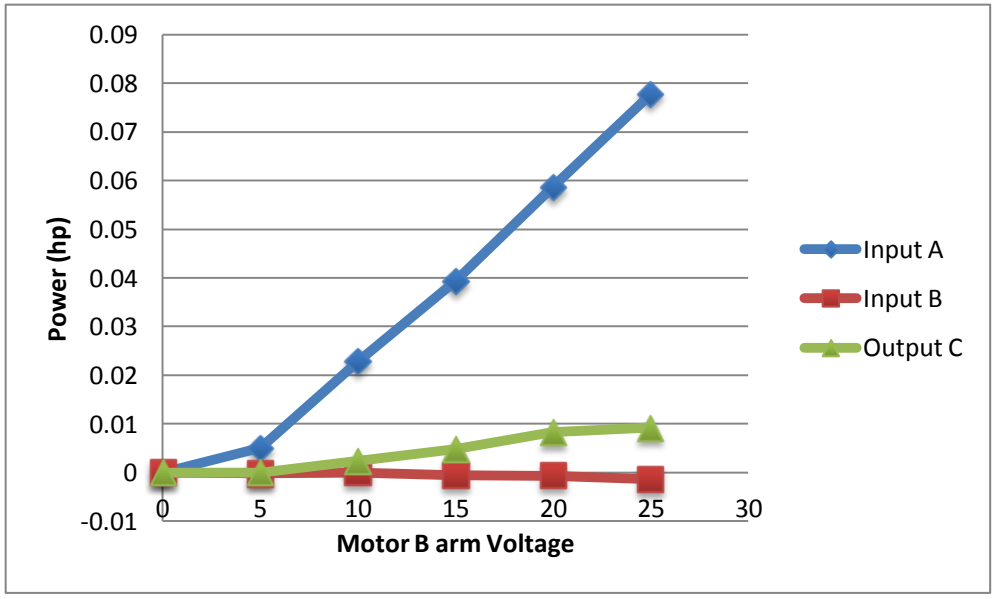

(a) GTD

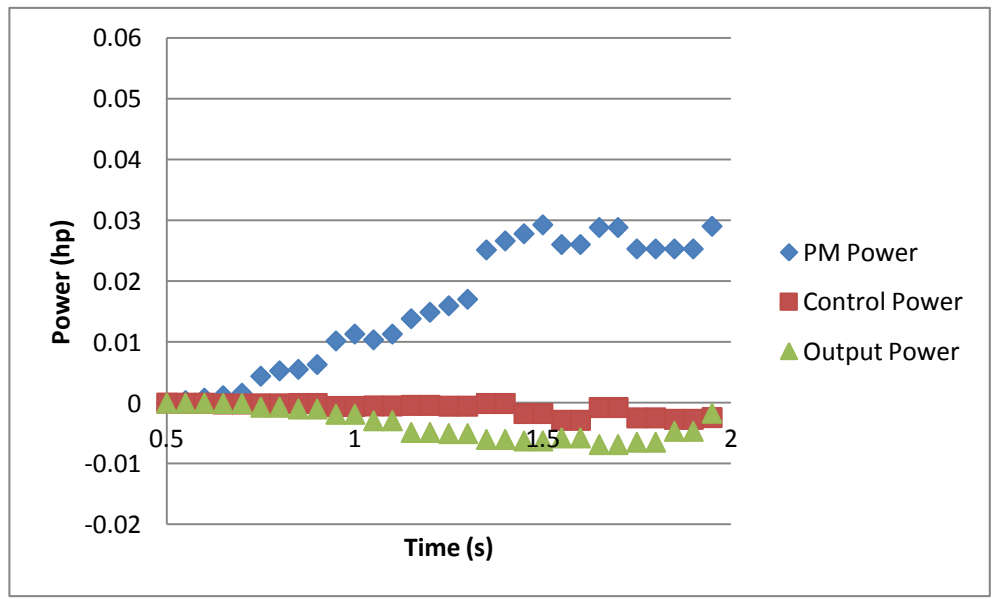

(b) PD

Figure 4.14: Test 2o - Comparison of Power 


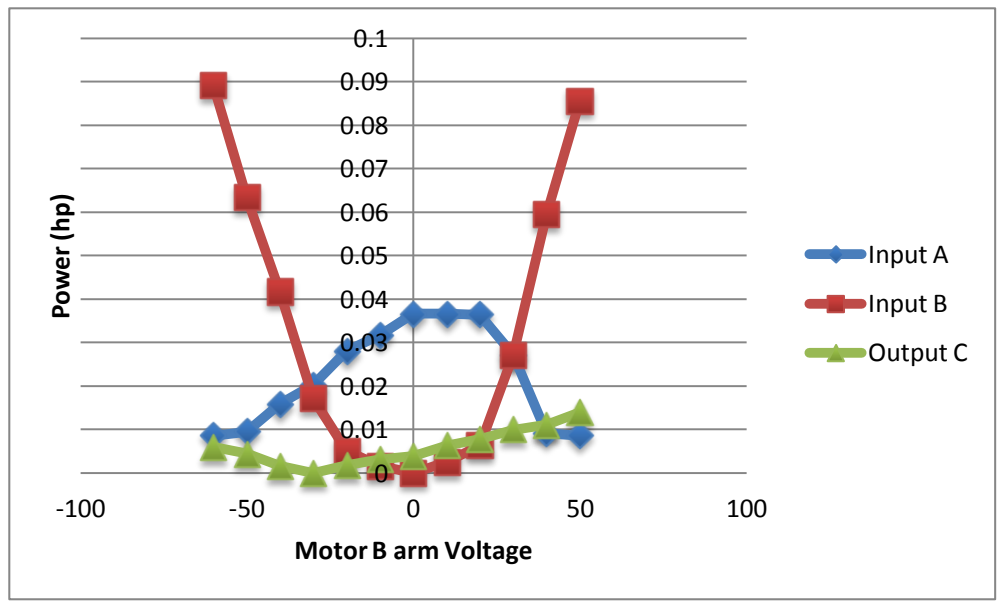

(a) GTD

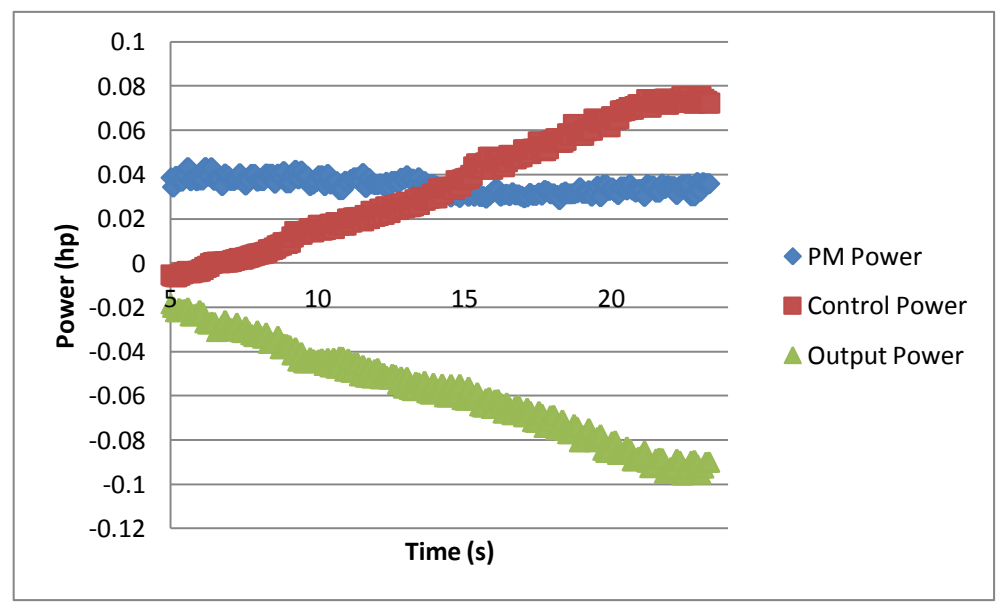

(b) PD

Figure 4.15: Test Crossover - Comparison of Power 


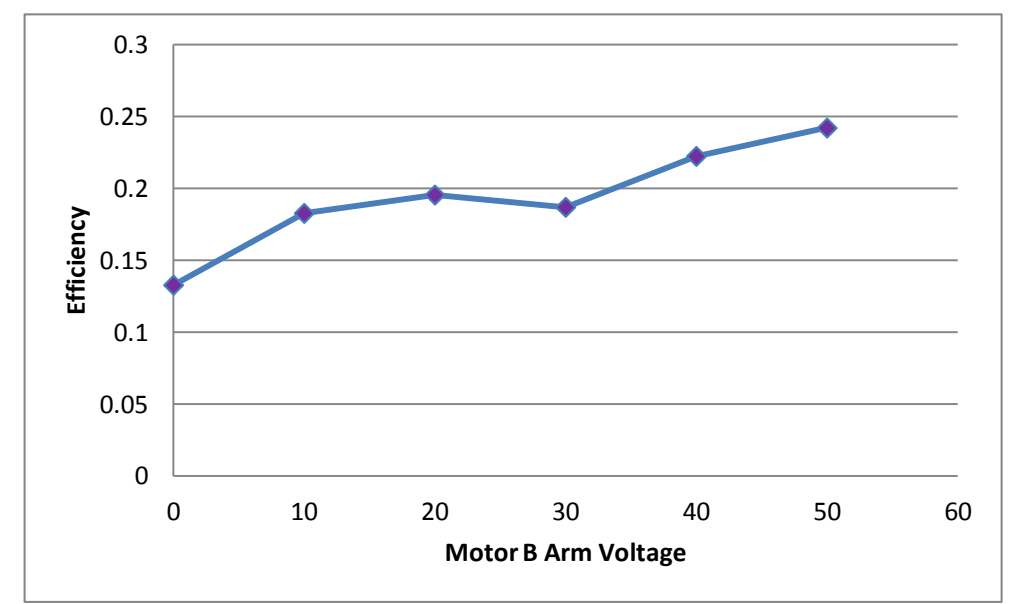

(a) GTD

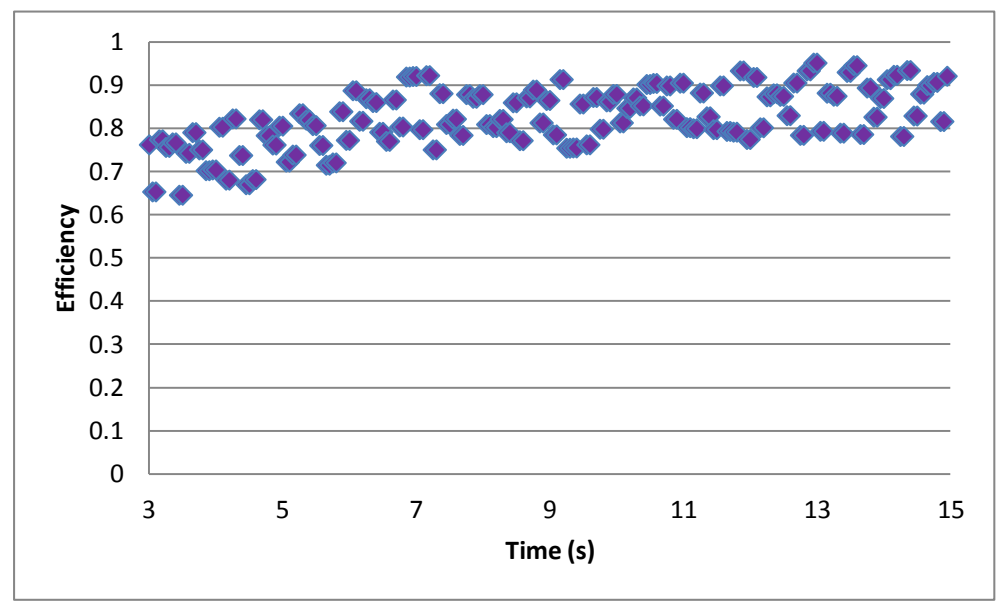

(b) PD

Figure 4.16: Test 1s - Comparison of Efficiency

\subsubsection{Efficiency Measurements}

This section shows the efficiency of both the GTD and PD by using the Figure 4.11 through 4.15 and Equation (2.12). Figures 4.16 through 4.20 display the results. These results show a wide variety of efficiencies depending on speed of each of the I/Os for the GTD and PD. This is due mostly to the nature of the tests performed. In Test 1s, the RPM of Input A remained constant, while the RPM of Input B started from zero and increased in the positive direction. Different speeds during testing was one of the main contributors in changing efficiency and will be explained more in a later section. 
In Test 1o, although the RPM of Input A remained constant, while the RPM of Input B started from zero and increased, Input B increased in the negative direction, which caused Output $\mathrm{C}$ to decrease in speed. This difference in direction from Input B enables the system to effectively be back-driven, thus reducing the efficiency of the system significantly, which characteristic was noted by Wells [3]. It can also be noted that both systems reached zero efficiency in these tests when zero velocity was seen at Output C, which property is inherent in Equations (2.12) and (4.3).

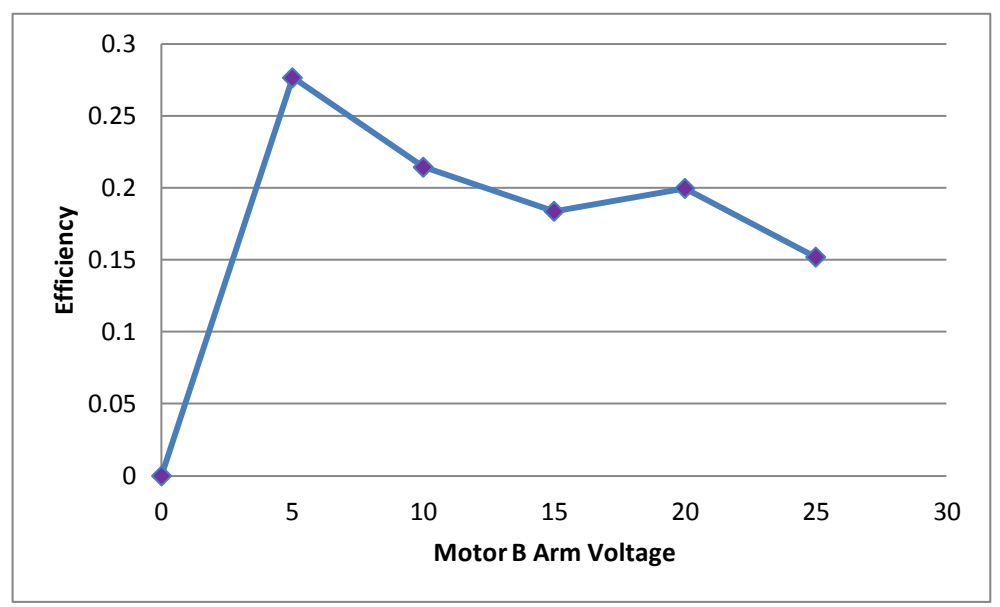

(a) GTD

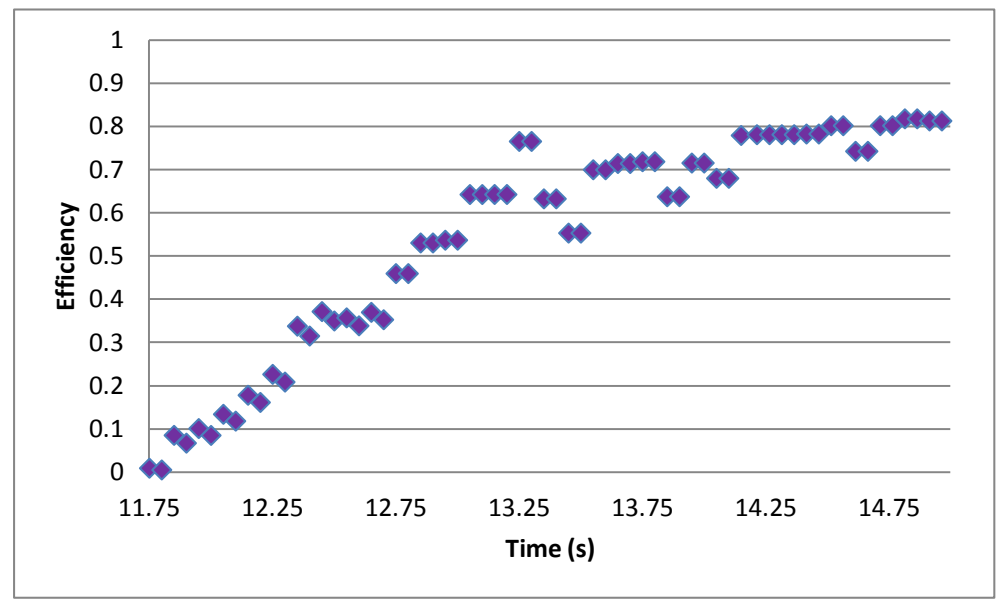

(b) PD

Figure 4.17: Test 2s - Comparison of Efficiency 


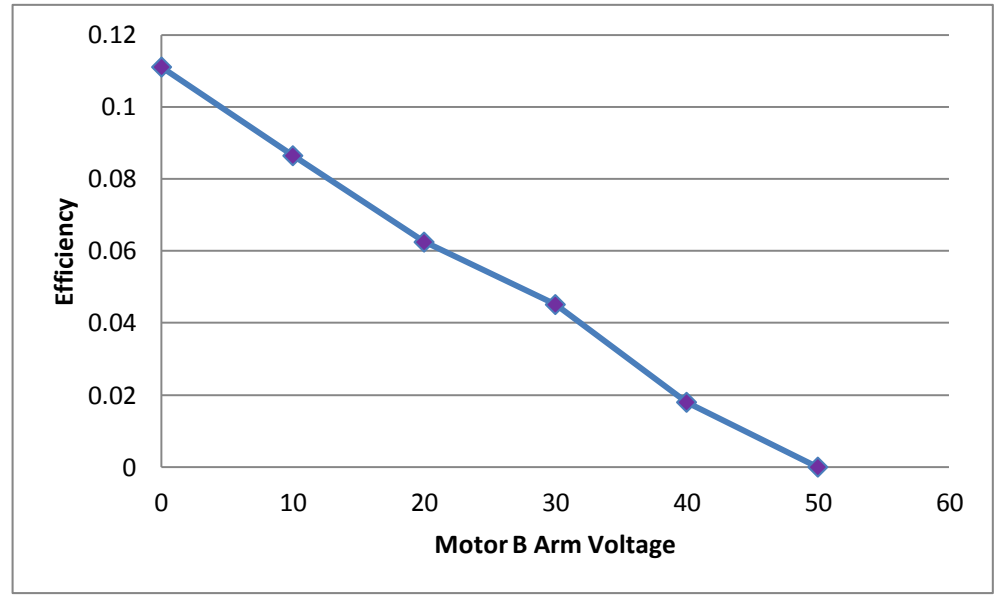

(a) GTD

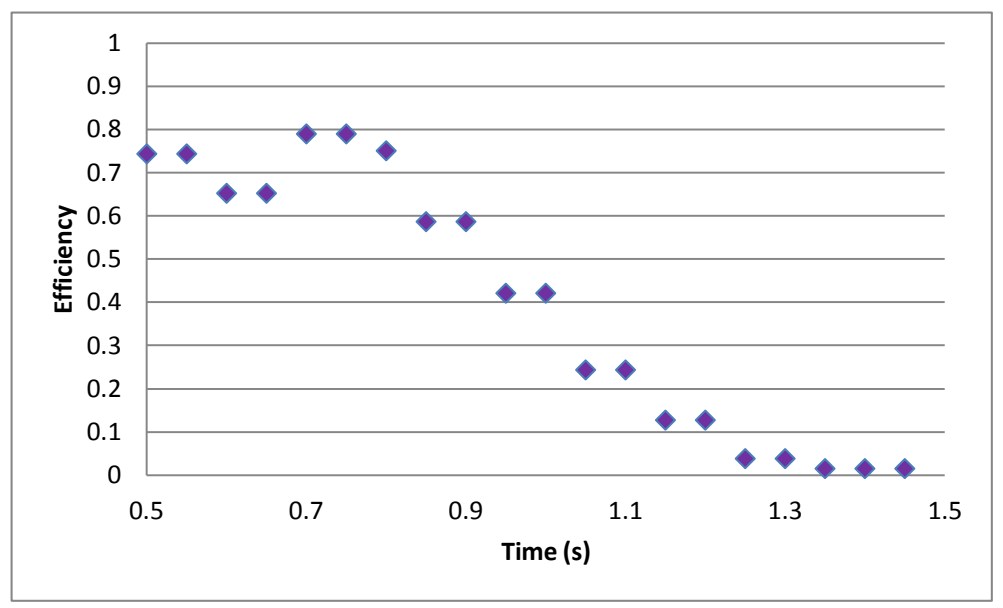

(b) PD

Figure 4.18: Test 1o - Comparison of Efficiency 


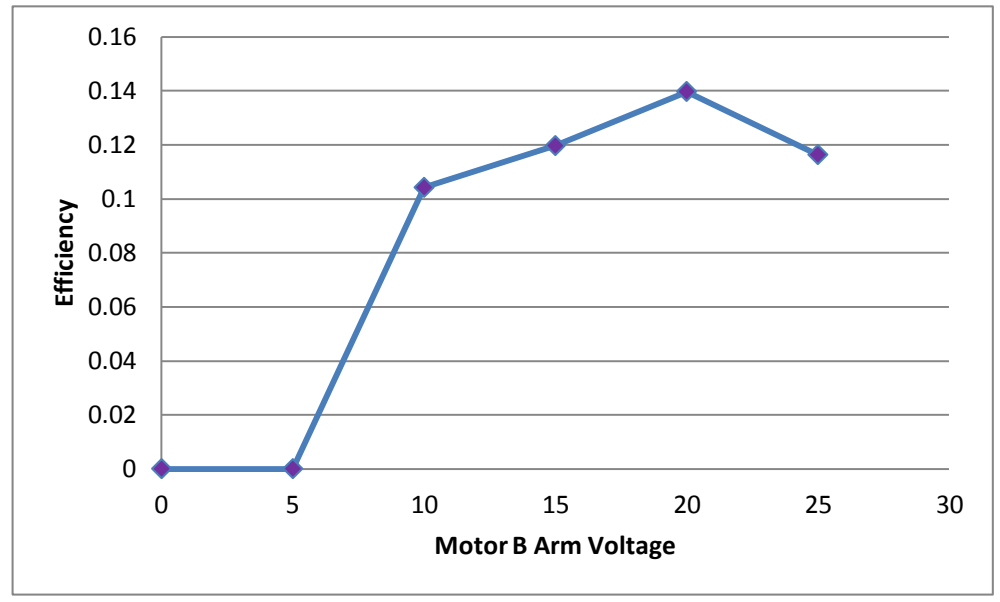

(a) GTD

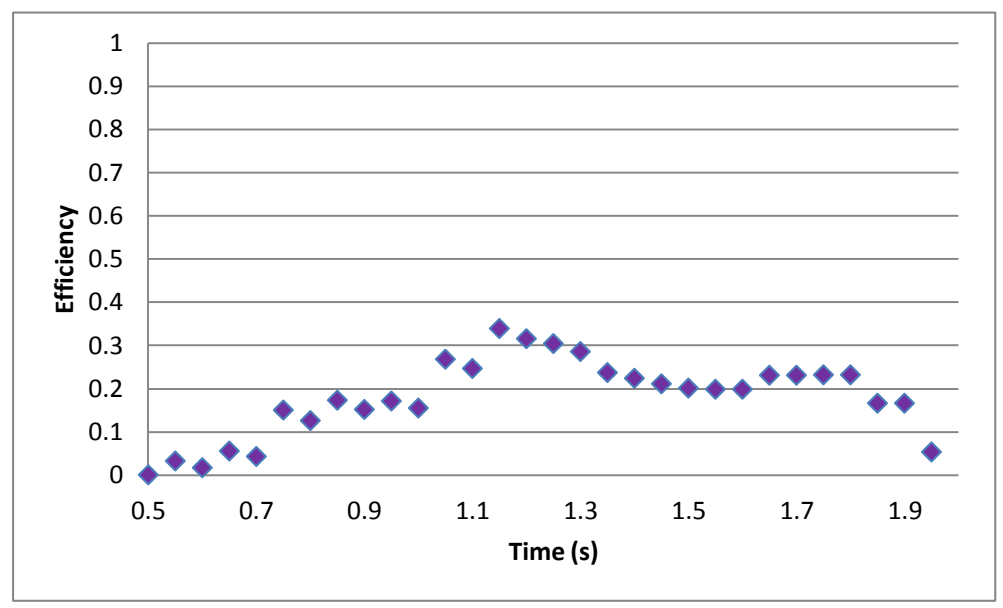

(b) $\mathrm{PD}$

Figure 4.19: Test 2o - Comparison of Efficiency 


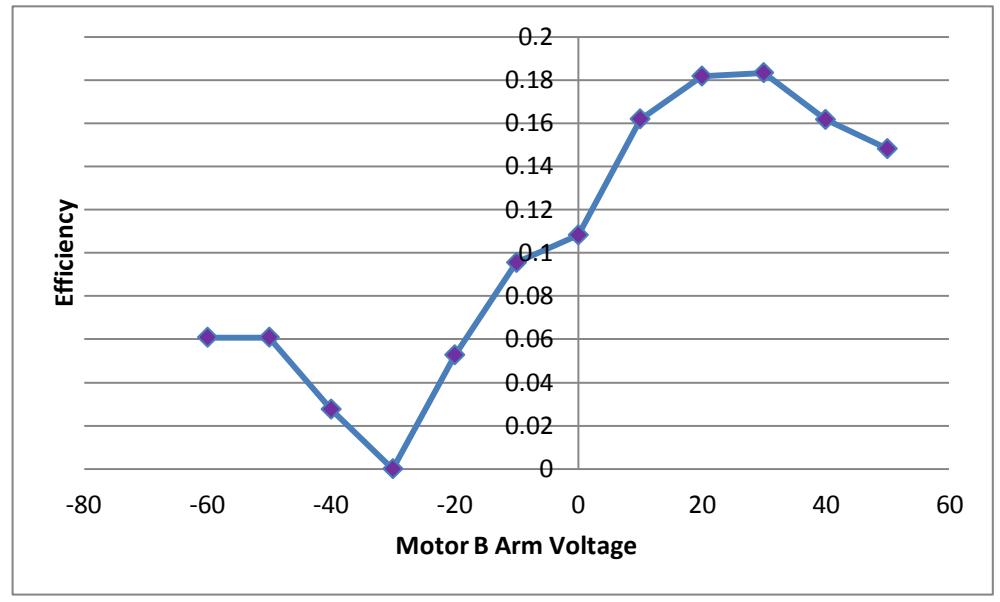

(a) GTD

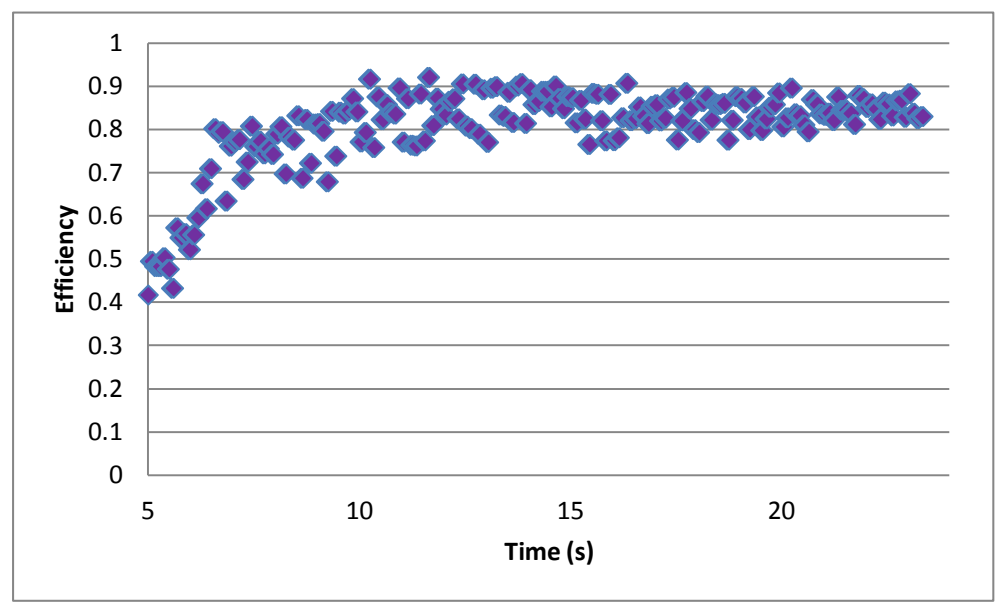

(b) PD

Figure 4.20: Test Crossover - Comparison of Efficiency 


\subsubsection{Implications}

A general look at calculated efficiency will help explain more of the results seen in the efficiency graphs. As efficiency tended to change significantly during each of the tests, Table 4.1 shows a head-to-head comparison of both the GTD and PDs' highest efficiencies obtained from the highest data point during each of the five tests. The lower efficiency of the GTD is due mostly to the mechanical nature of the gears used in the GTD and was anticipated, as explained earlier in this work. The GTD was never designed to be directly used as a dual-input single-output device. The PD used in this research, on the other hand, was designed for this purpose and exhibits a much higher efficiency in all 5 of these tests. These results show that the PD has greater potential as a solution for use as a CVT in automotive applications.

Table 4.1: Highest Achieved Efficiency Comparison

\begin{tabular}{|c|c|c|c|}
\hline Test Name & GTD (\%) & PD (\%) & Improvement (\%) \\
\hline 1s & 24.194 & 93.263 & 69.069 \\
\hline 2s & 27.654 & 81.198 & 53.544 \\
\hline 1o & 11.111 & 78.908 & 67.797 \\
\hline 2o & 13.968 & 33.869 & 19.901 \\
\hline Crossover & 18.333 & 91.986 & 73.653 \\
\hline Average & $\mathbf{1 9 . 0 5 2}$ & $\mathbf{7 5 . 8 5 1}$ & $\mathbf{5 6 . 7 9 9}$ \\
\hline
\end{tabular}

\subsection{Additional Testing}

In addition to the first 5 tests, it was determined that the appropriate solution needed to be tested a little more in-depth than the GTD was tested. To do this a series of tests, where both the RPM and Load vary to allow for more realistic testing scenarios, was selected. The following 9 tests describe how the Planetary Differential was tested.

- High RPM, High Load (HH)

- High RPM, Medium Load (HM)

- High RPM, Low Load (HL) 
- Medium RPM, High Load (MH)

- Medium RPM, Medium Load (MM)

- Medium RPM, Low Load (ML)

- Low RPM, High Load (LH)

- Low RPM, Medium Load (LM)

- Low RPM, Low Load (LL)

The two capital letters that appear next to each of these 9 tests are their respective abbreviations, where the first letter indicates the RPM and the second letter indicates the Load. Table 4.2 shows the numerical values represented by High, Medium and Low for both RPM and Load. These abbreviations will be used for simplification in referring to each of these additional tests.

Table 4.2: Numerical Values for RPM and Load Variations

\begin{tabular}{|c|c|c|c|}
\hline Test Parameter & Abbreviation & RPM & Load (in-lb) \\
\hline High & H & 300 & 30 \\
\hline Medium & M & 200 & 20 \\
\hline Low & L & 100 & 10 \\
\hline
\end{tabular}

\subsubsection{RPM Measurements}

This section shows RPM measurements in testing the PD under all 9 tests (Figures 4.21, 4.22 and 4.23). In Figure 4.21 the first input (PM Speed) remained constant at 300 RPM, while the second input (Control Speed) varied from a negative RPM to a positive RPM. Each of these tests in Figure 4.21 show a slightly different result as the variation of Control Speed had a different range. In Test HH, Control Speed varied from about -200 to around -100 RPM. In Test HM, Control Speed varied from about -150 to around 300 RPM. In Test HL, Control Speed varied from about -100 to around 300 RPM. The difference in starting RPM and the ability to vary RPM for the Control Speed is due to the back-drive-able nature of $3 \mathrm{I} / \mathrm{O}$ devices. With higher load, the Control Speed range decreases. 
This trend can be seen in all the higher load tests (Tests that have an " $\mathrm{H}$ " in the second abbreviation location) as increased load decreases the range and capability of the Control Speed Input. This problem of back-drive-ability is the topic of more in-depth research and is currently under investigation. A purpose of this research is not to fully address it, but simply recognize that it exists and that a solution is being investigated.

\subsubsection{Torque Measurements}

This section shows torque measurements in testing the PD under all 9 tests (Figures 4.24, 4.25 and 4.26). The torque data in these graphs demonstrates the PD's compliance to Equation (4.2). It can be seen from Test HL, for example, that the sum of all 3 measured torque values is approximately equal to zero. This trend, although not perfect due to efficiency losses, can be seen in all 9 tests in Figures 4.24, 4.25 and 4.26. 


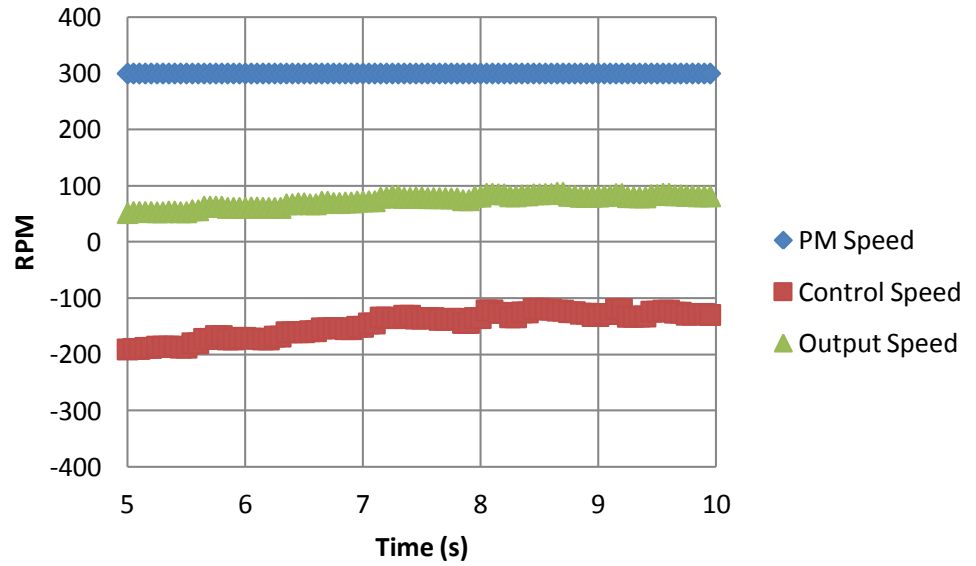

(a) $\mathrm{HH}$

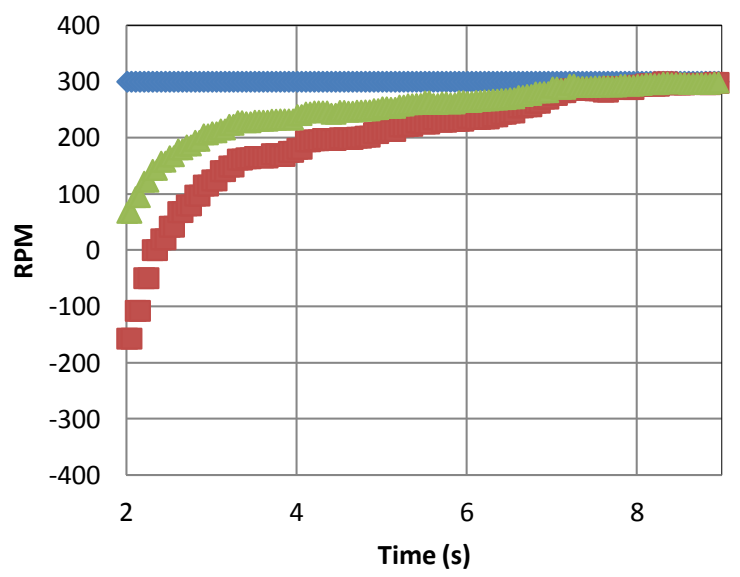

$\checkmark$ PM Speed

Control Speed

$\triangle$ Output Speed

(b) $\mathrm{HM}$

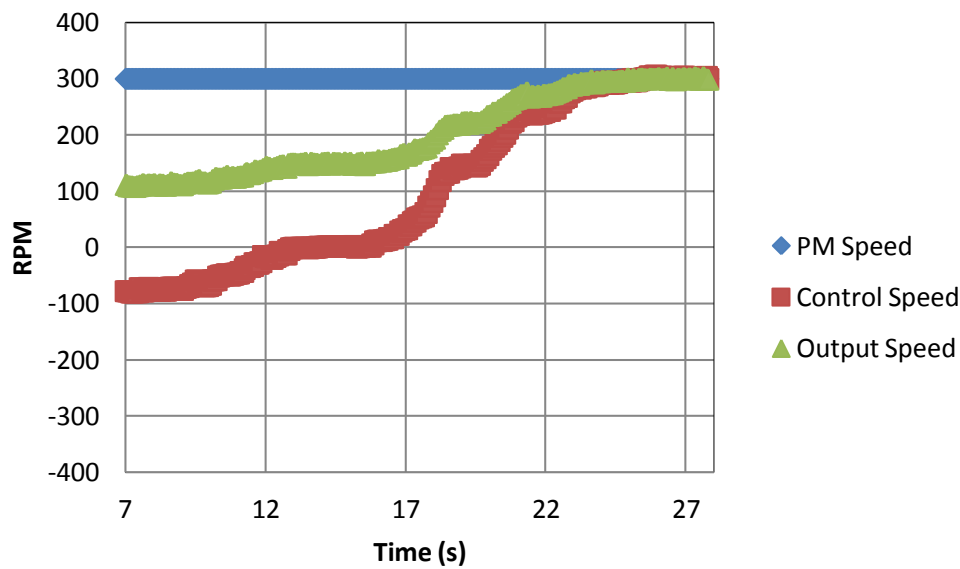

(c) $\mathrm{HL}$

Figure 4.21: RPM Measurements at High RPM with Varying Loads 


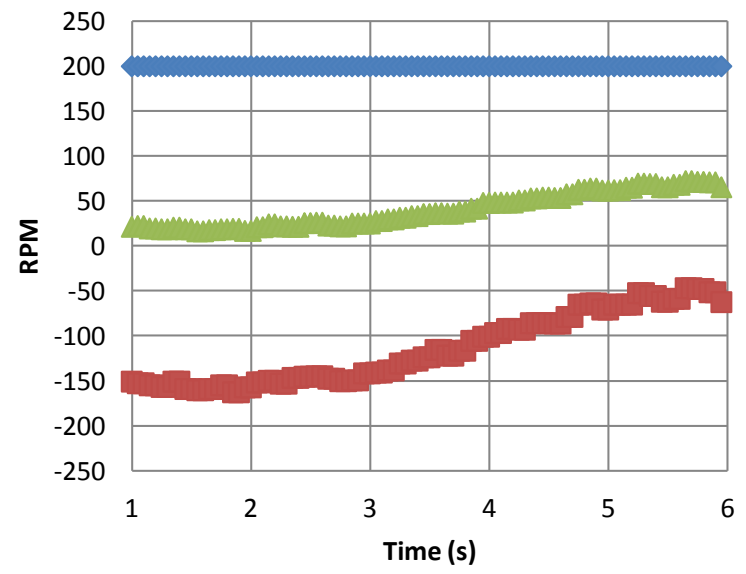

PM Speed

Control Speed

$\triangle$ Output Speed

(a) $\mathrm{MH}$

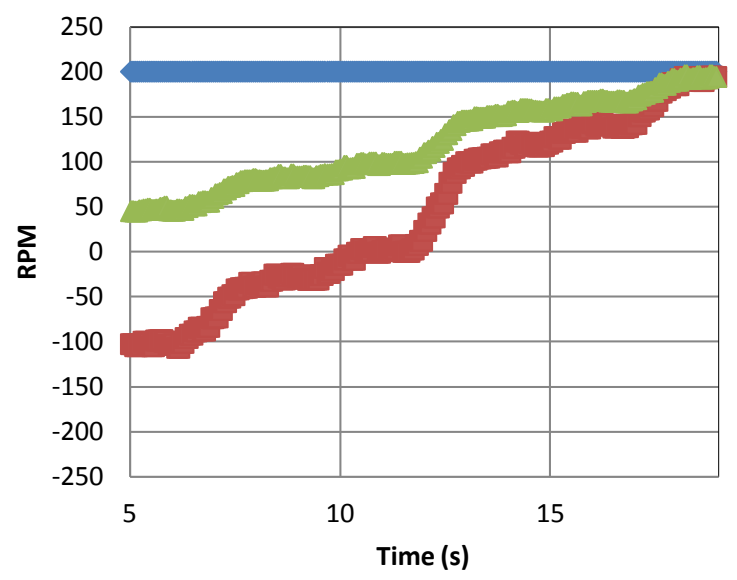

PM Speed

Control Speed

$\triangle$ Output Speed

(b) $\mathrm{MM}$

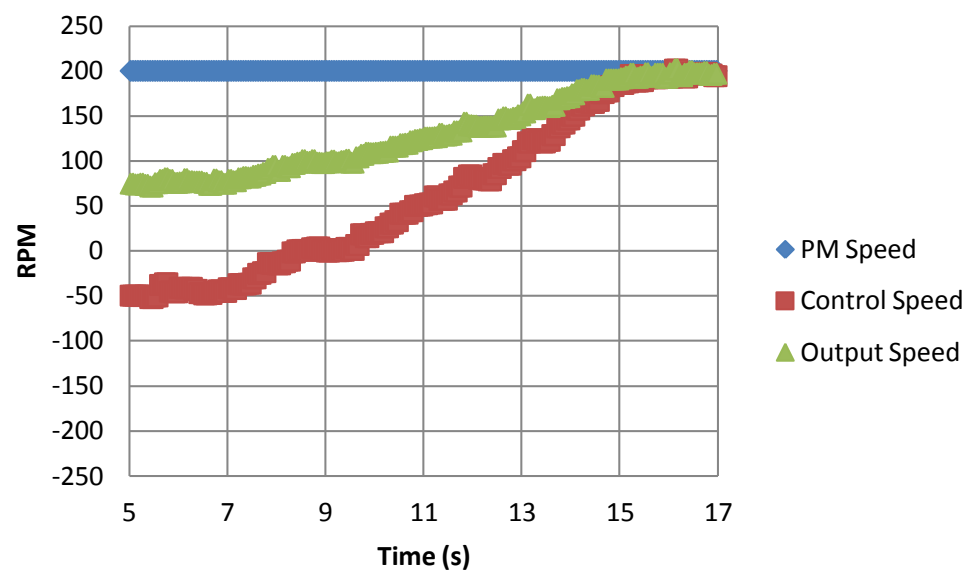

(c) ML

Figure 4.22: RPM Measurements at Medium RPM with Varying Loads 


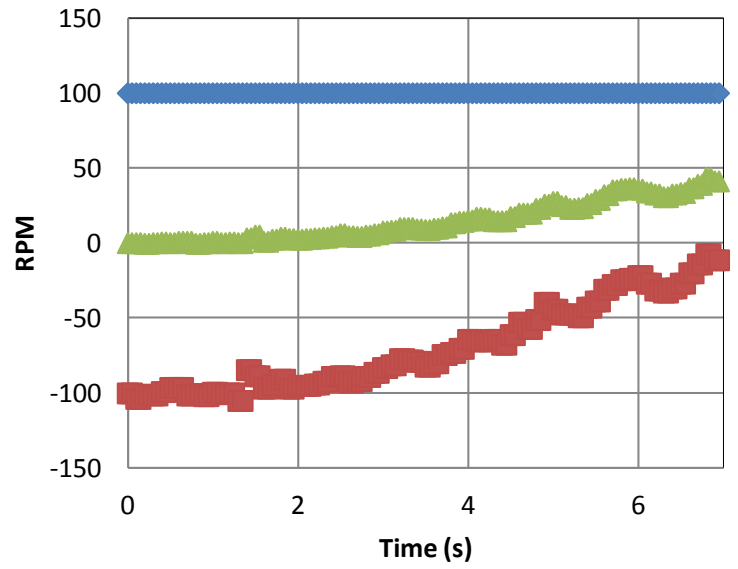

PM Speed

Control Speed

$\triangle$ Output Speed

(a) $\mathrm{LH}$

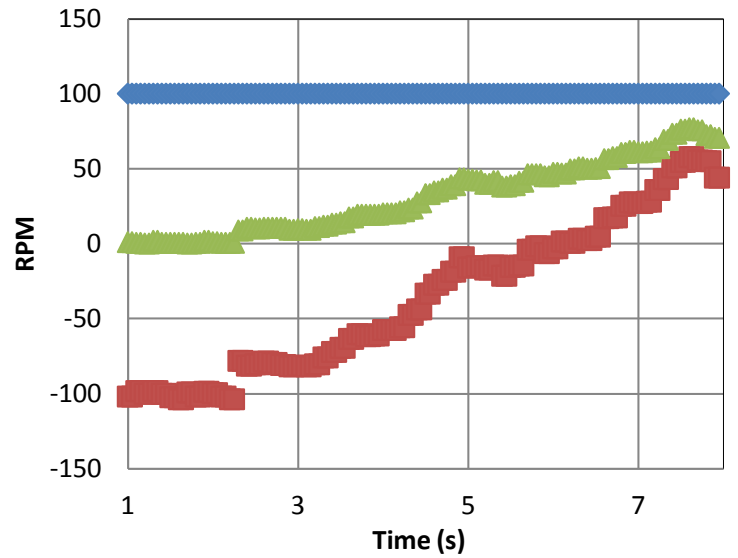

$\diamond$ PM Speed

Control Speed

$\triangle$ Output Speed

(b) LM

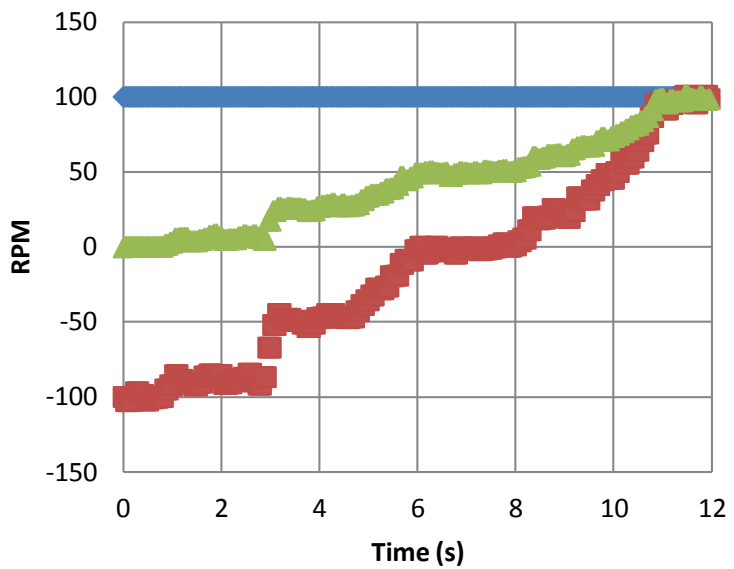

$\checkmark$ PM Speed

Control Speed

$\triangle$ Output Speed

(c) $\mathrm{LL}$

Figure 4.23: RPM Measurements at Low RPM with Varying Loads 


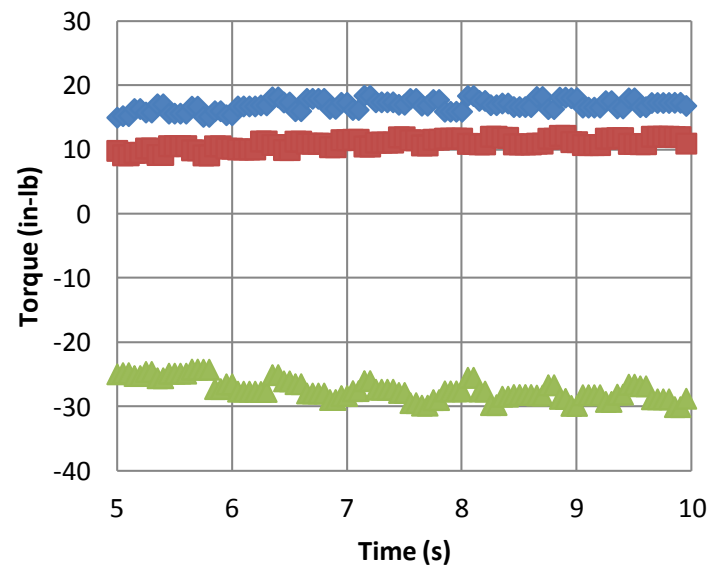

- Interface Torque

Omega Torque

$\triangle$ Futek Torque

(a) $\mathrm{HH}$

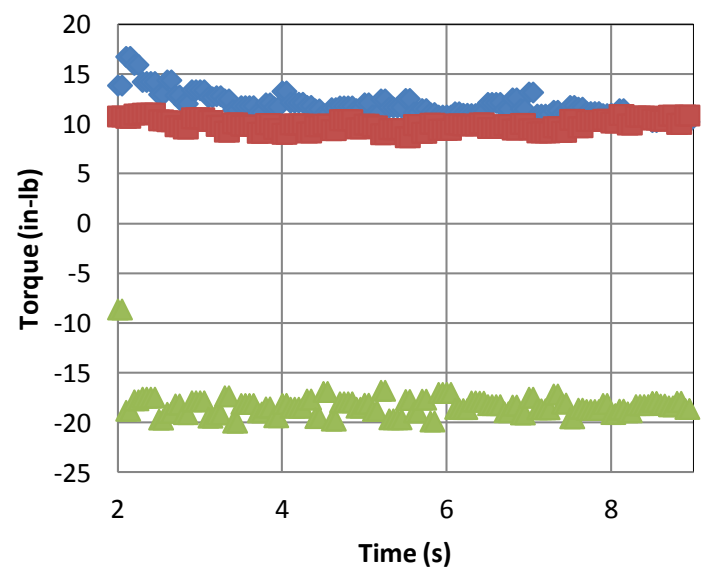

$\checkmark$ Interface Torque

Omega Torque

$\triangle$ Futek Torque

(b) $\mathrm{HM}$

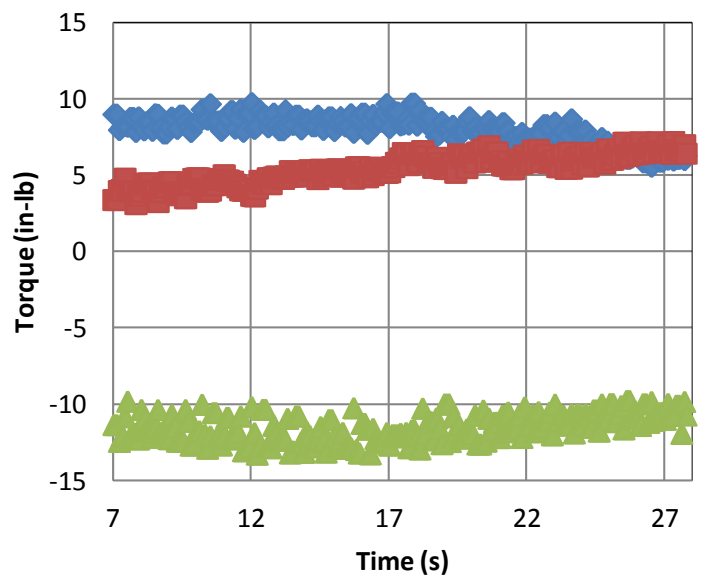

Interface Torque

Omega Torque

$\triangle$ Futek Torque

(c) $\mathrm{HL}$

Figure 4.24: Torque Measurements at High RPM with Varying Loads 


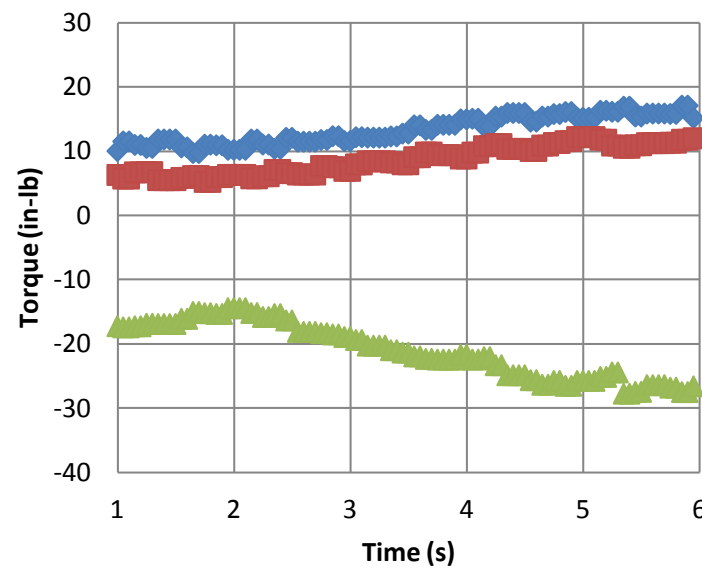

Interface Torque

Omega Torque

$\triangle$ Futek Torque

(a) $\mathrm{MH}$

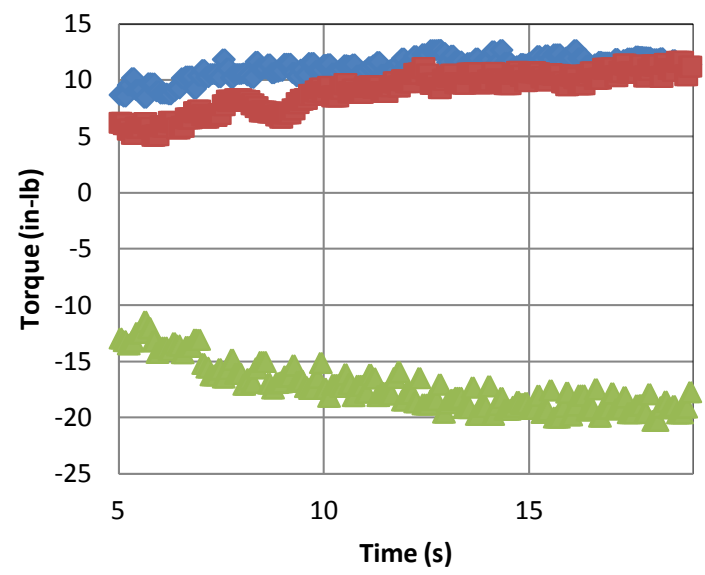

Interface Torque

Omega Torque

$\triangle$ Futek Torque

(b) $\mathrm{MM}$

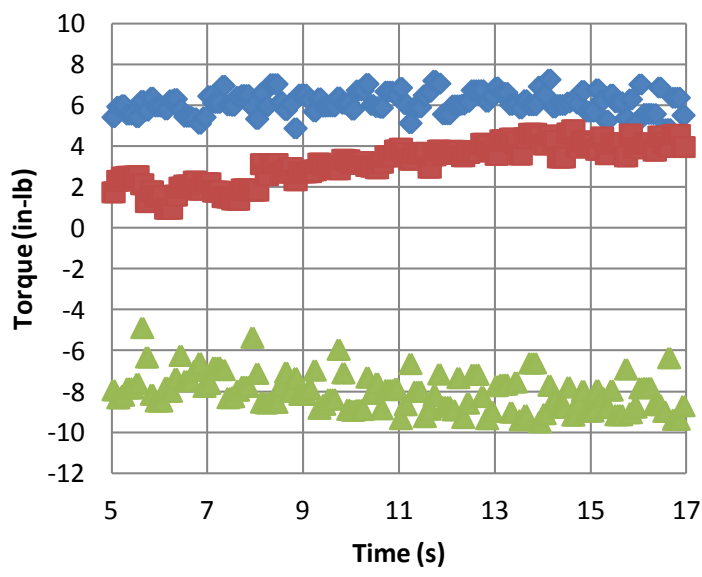

Interface Torque

Omega Torque

$\triangle$ Futek Torque

(c) ML

Figure 4.25: Torque Measurements at Medium RPM with Varying Loads 


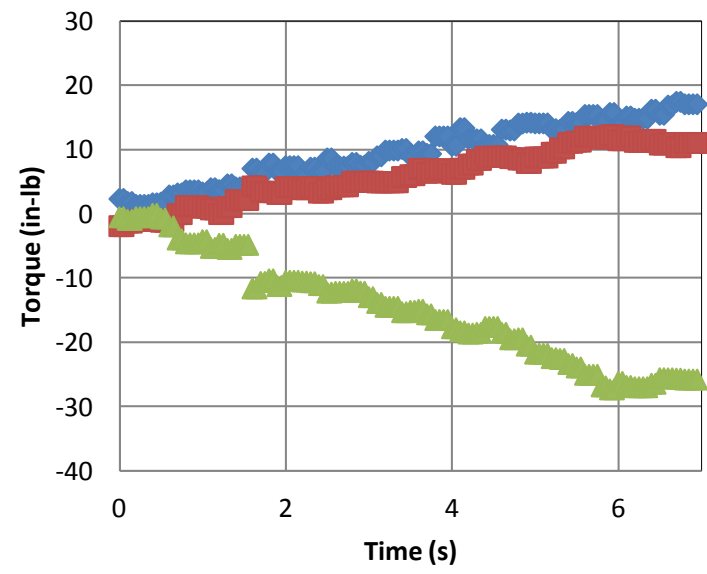

- Interface Torque

Omega Torque

$\triangle$ Futek Torque

(a) $\mathrm{LH}$

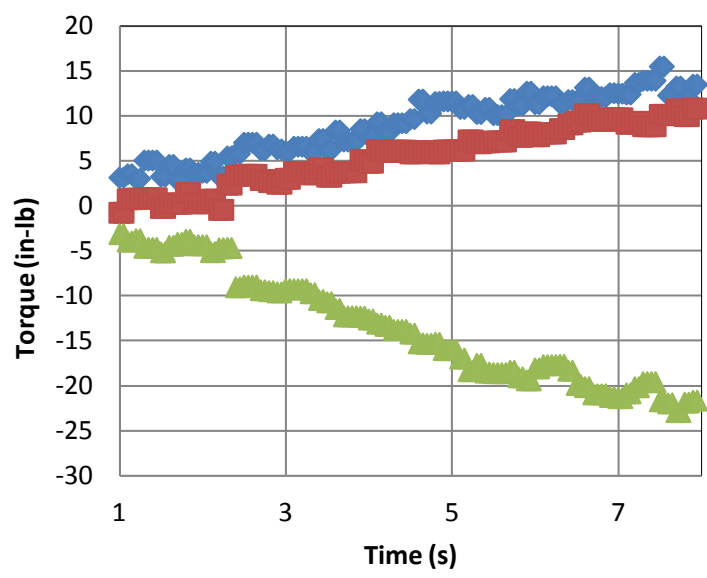

- Interface Torque

Omega Torque

$\triangle$ Futek Torque

(b) LM

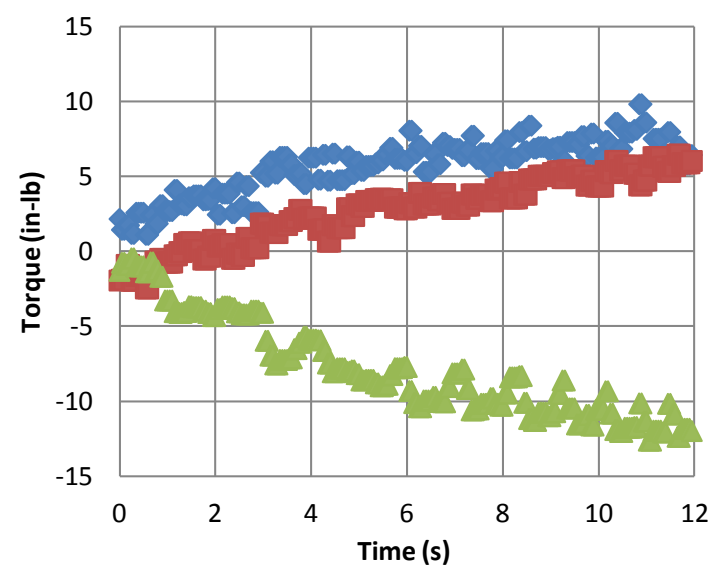

$\diamond$ Interface Torque

- Omega Torque

$\triangle$ Futek Torque

(c) LL

Figure 4.26: Torque Measurements at Low RPM with Varying Loads 


\subsubsection{Power Measurements}

This section shows power measurements in testing the PD under all 9 tests (Figures 4.27, 4.28 and 4.29). Power for these graphs is measured in horsepower, again using Equation (2.1). Variation of load in these graphs plays a significant role in producing different results. It can be observed from the higher load tests that Control Power and Output Power swap positions vertically through the duration of the test. This result is due to the Control Motor from the PD beginning to be back-driven by the load at the Output. The Control Motor power is limited in range by the output load.

\subsubsection{Efficiency Measurements}

This section shows efficiency measurements in testing the PD under all 9 tests (Figures 4.30, 4.31 and 4.32). This data shows that efficiency increased as speed of the Control Motor drew closer to the constant speed of the PM. It can be observed that there are imperfections in the assembly of this system, which are assumed to be one of the causes of this inefficiency difference at different speeds. It is assumed that as the system gains rotational motion, the binding felt in one or more of the gear meshes is overcome proportionally with a continuing increase of speed. In a professionally manufactured PD, this result would be minimized, but for the purpose of this research the efficiency percentages from this data suffice as a promising result. 


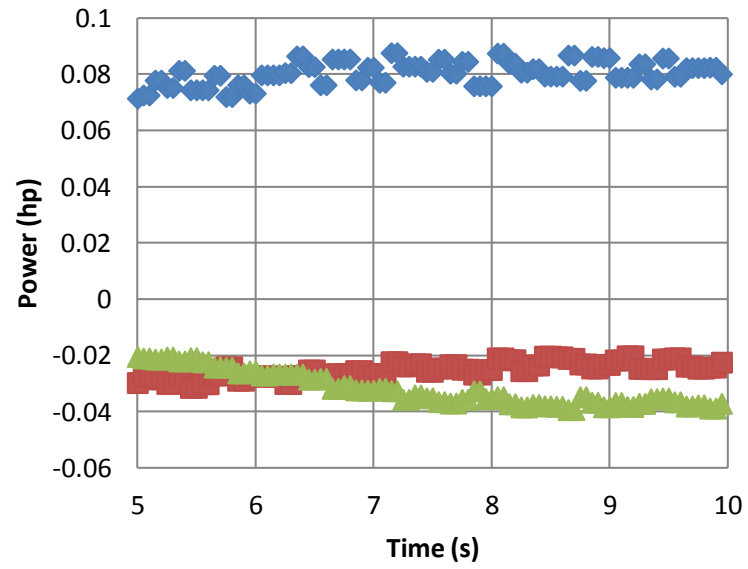

- PM Power

Control Power

$\triangle$ Output Power

(a) $\mathrm{HH}$

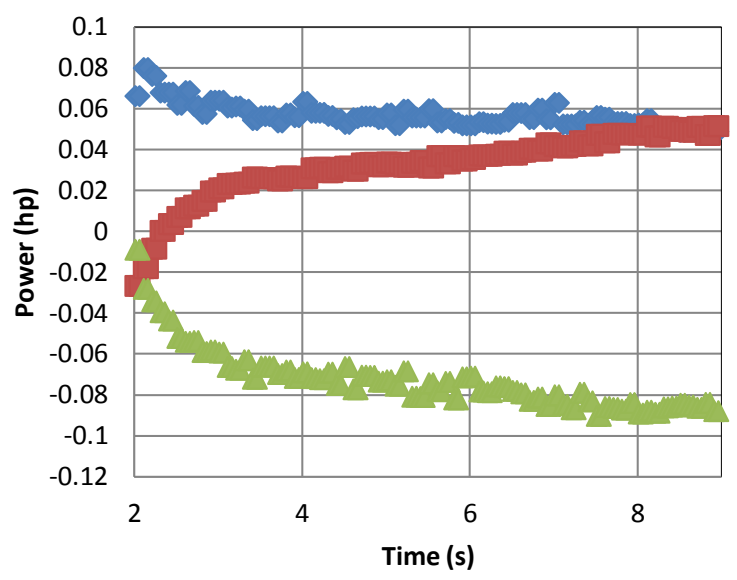

- PM Power

Control Power

$\triangle$ Output Power

(b) $\mathrm{HM}$

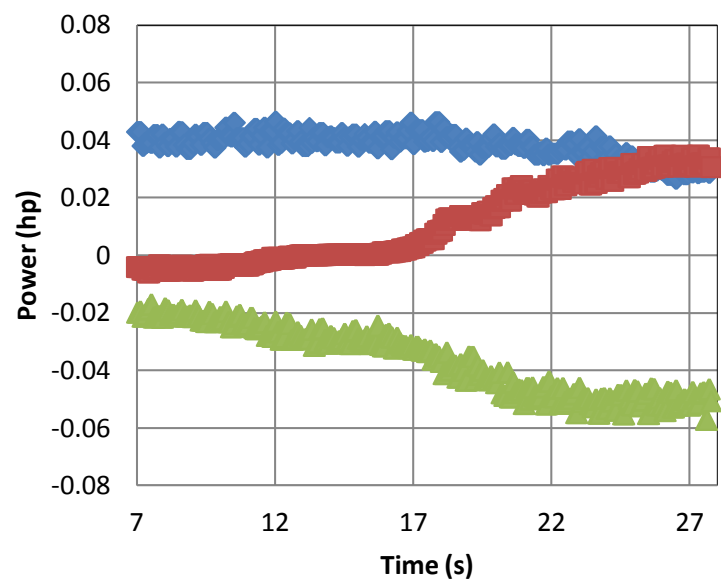

$\rightarrow$ PM Power

Control Power

$\triangle$ Output Power

(c) $\mathrm{HL}$

Figure 4.27: Power Measurements at High RPM with Varying Loads 


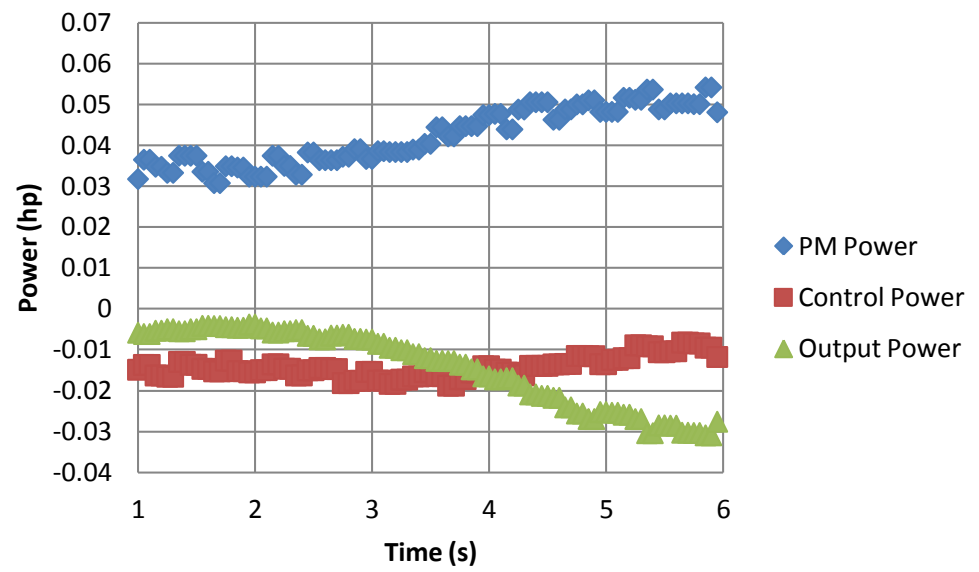

(a) $\mathrm{MH}$

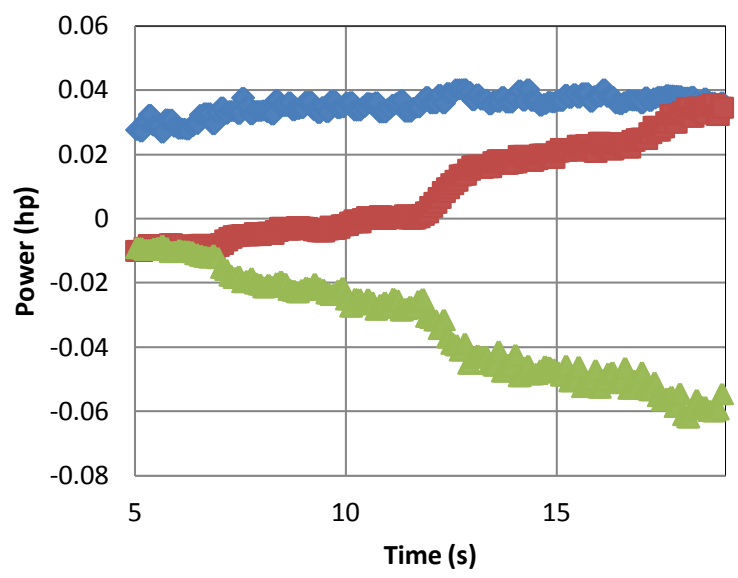

$\rightarrow$ PM Power

- Control Power

$\triangle$ Output Power

(b) $\mathrm{MM}$

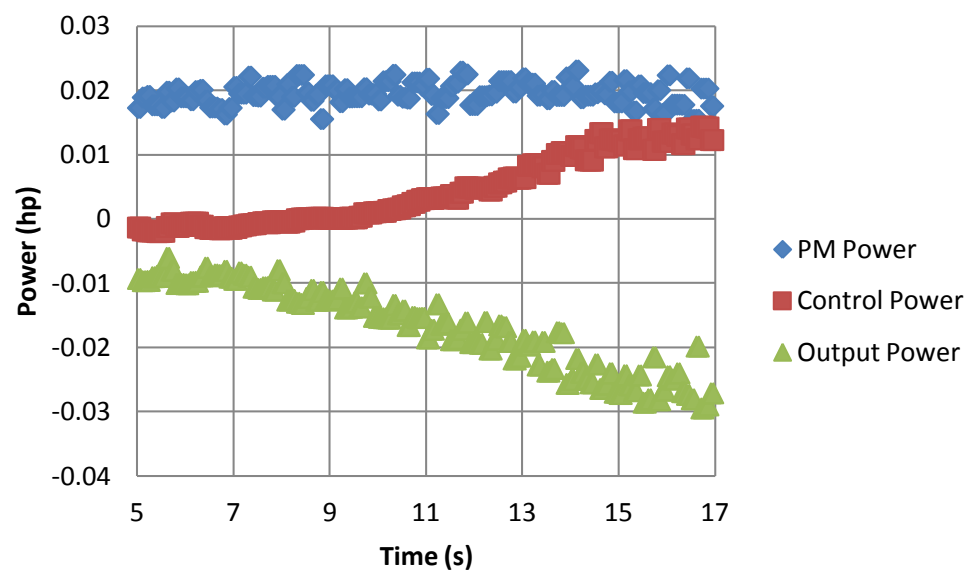

(c) $\mathrm{ML}$

Figure 4.28: Power Measurements at Medium RPM with Varying Loads 


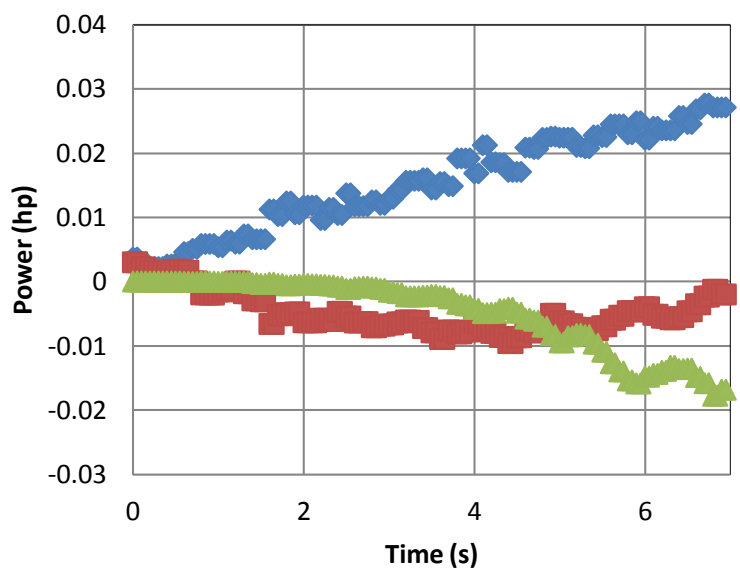

PM Power

Control Power

$\triangle$ Output Power

(a) $\mathrm{LH}$

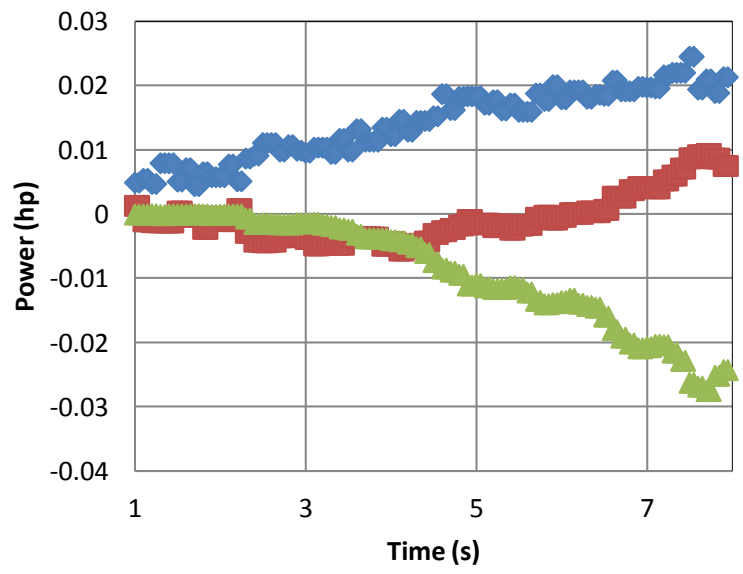

PM Power

Control Power

$\triangle$ Output Power

(b) LM

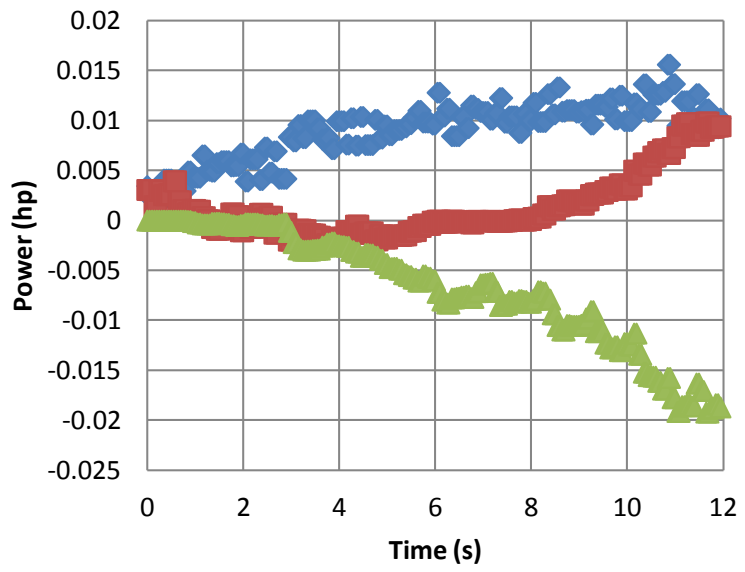

$\diamond$ PM Power

Control Power

$\triangle$ Output Power

(c) LL

Figure 4.29: Power Measurements at Low RPM with Varying Loads 


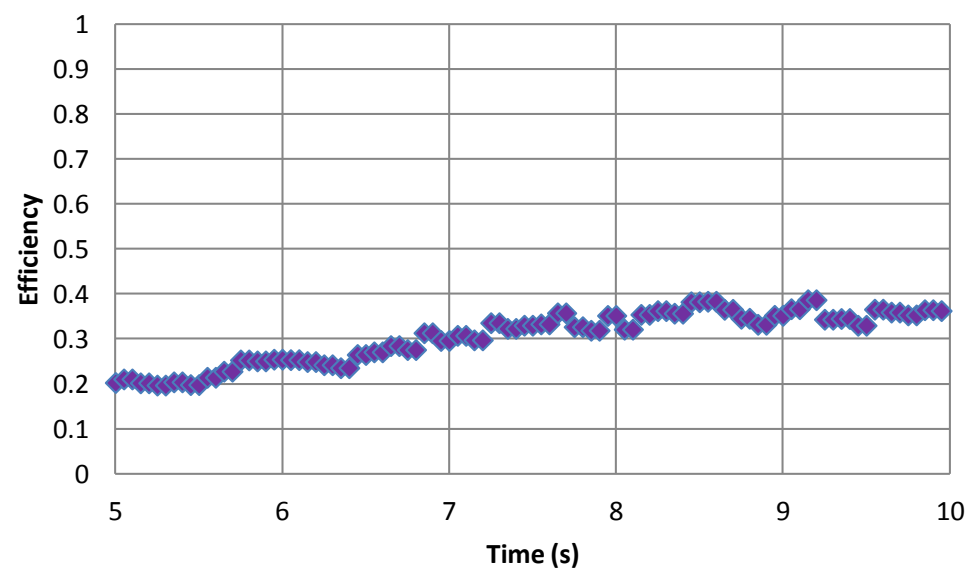

(a) $\mathrm{HH}$

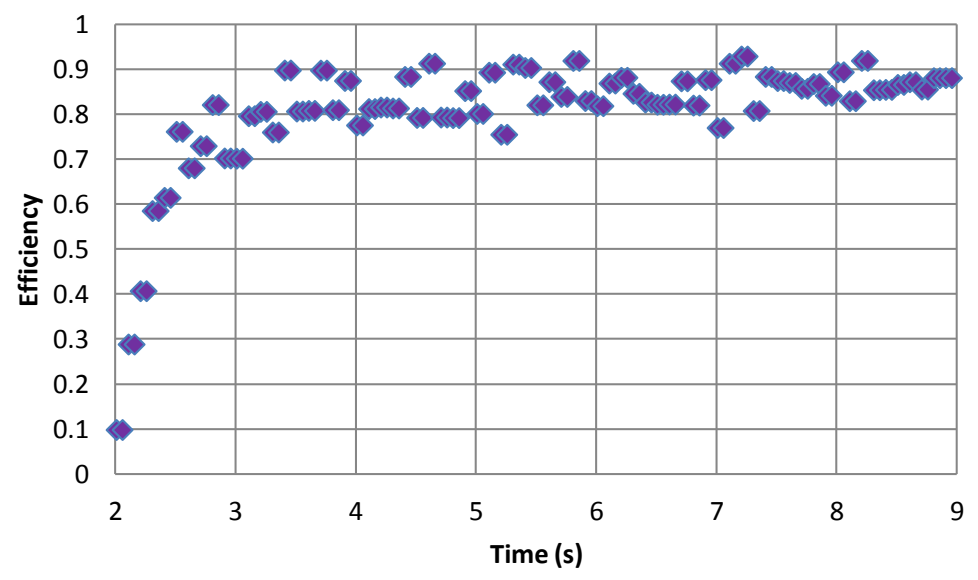

(b) $\mathrm{HM}$

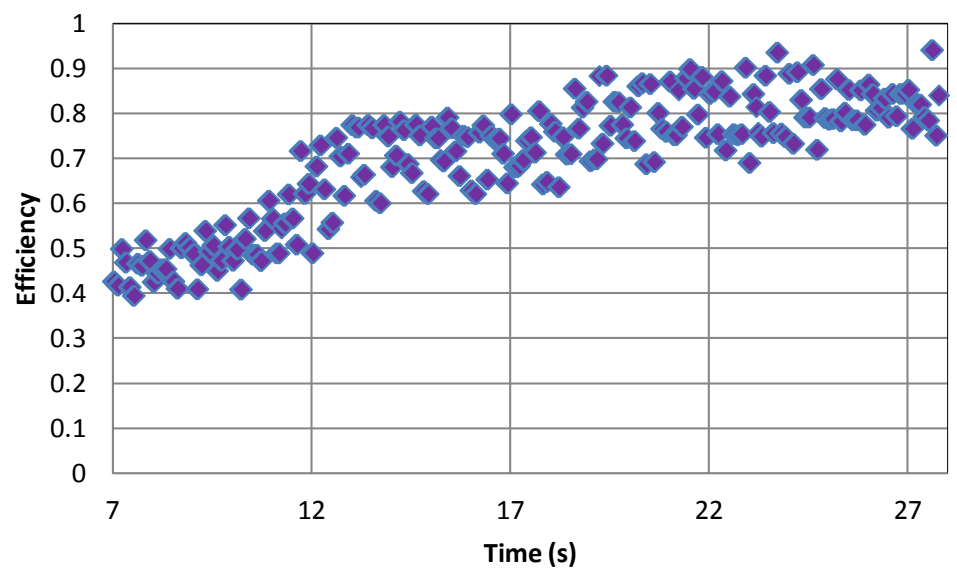

(c) HL

Figure 4.30: Efficiency Measurements at High RPM with Varying Loads 


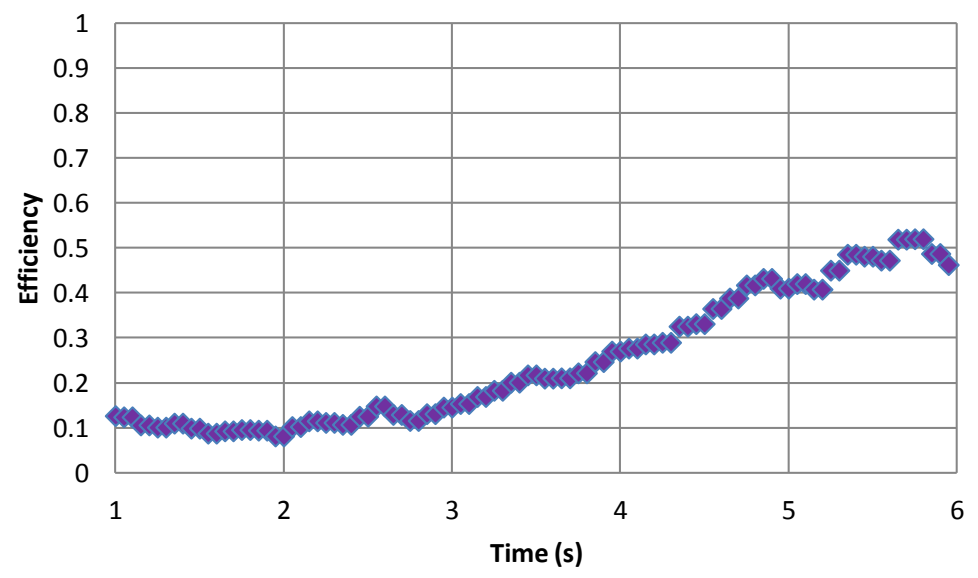

(a) $\mathrm{MH}$

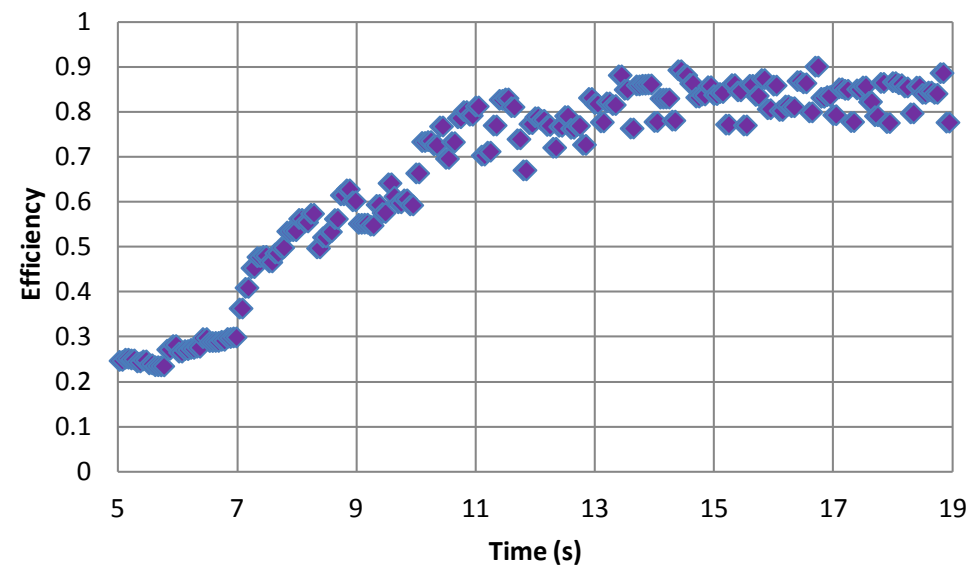

(b) $\mathrm{MM}$

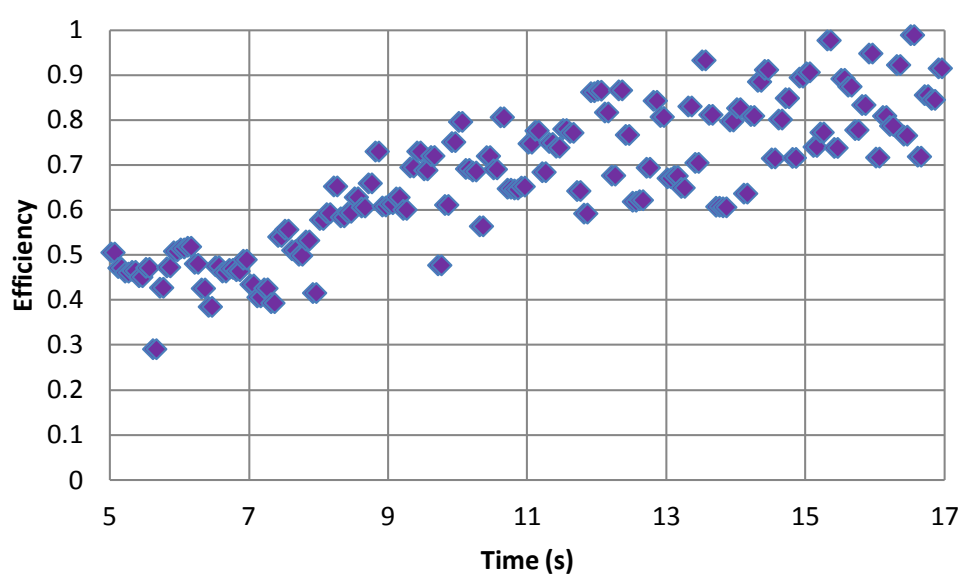

(c) $\mathrm{ML}$

Figure 4.31: Efficiency Measurements at Medium RPM with Varying Loads 


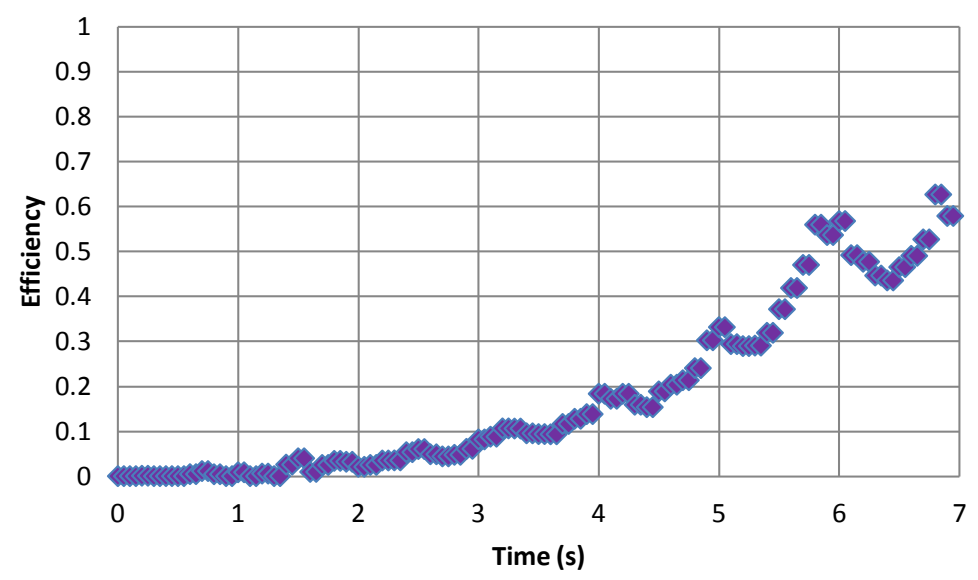

(a) $\mathrm{LH}$

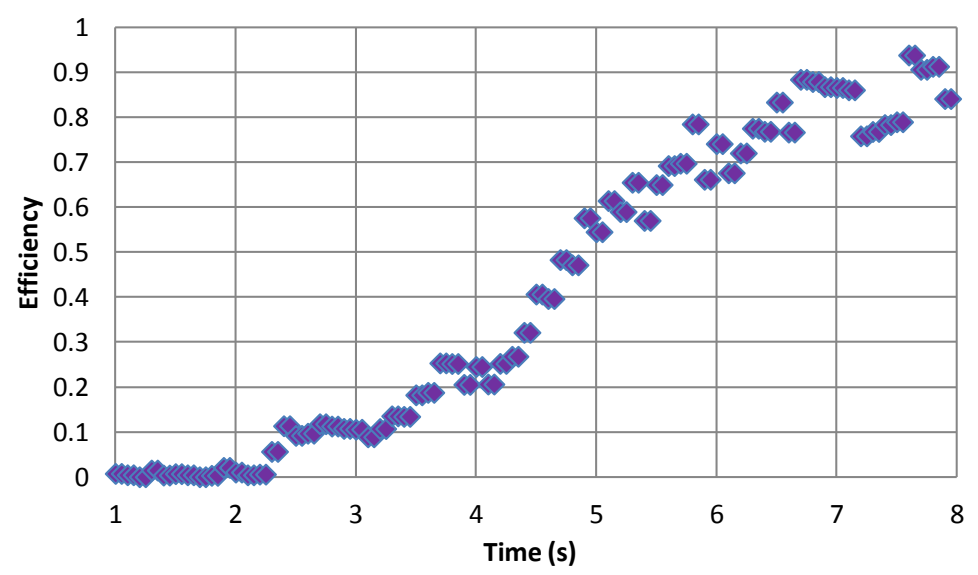

(b) LM

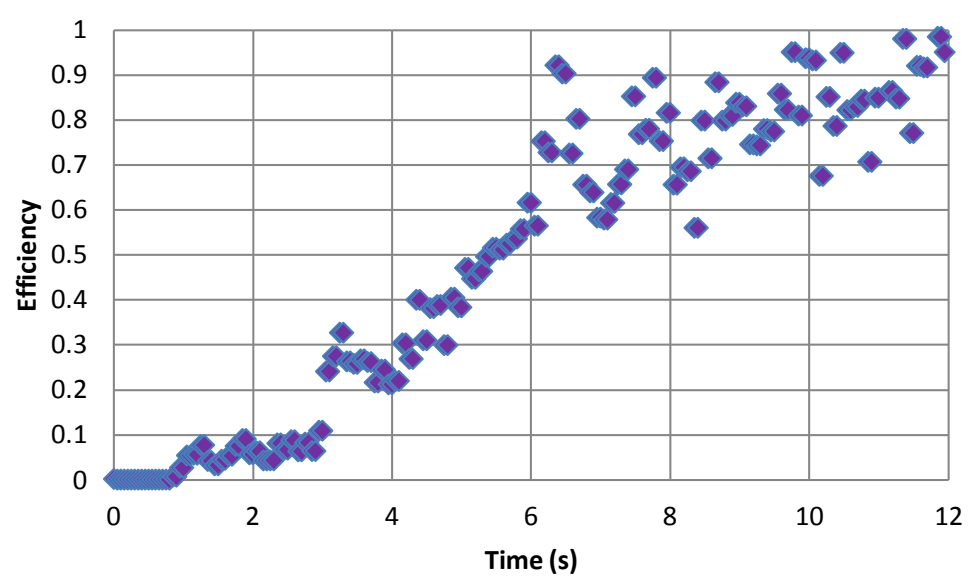

(c) LL

Figure 4.32: Efficiency Measurements at Low RPM with Varying Loads 


\subsubsection{Implications}

Table 4.3 shows the highest efficiency obtained for each of the 9 tests along with an average efficiency for all 9 tests. These results indicate that the PD would be a more efficient and, therefore, more appropriate solution for use as a CVT in automotive applications than the GTD. It also shows that small power can control large power, but in this case only to the degree that the Output load does not begin to back-drive the Control Motor input. The noise in the data acquisition can be seen in Table 4.3 by the $107.38 \%$ efficiency obtained in Test LL. Future testing would require more capable measuring devices to obtain more accurate data.

Table 4.3: Efficiency Comparison of all 9 Tests

\begin{tabular}{|c|c|}
\hline Test Name & Efficiency (\%) \\
\hline HH & 38.644 \\
\hline HM & 92.882 \\
\hline HL & 94.134 \\
\hline MH & 51.980 \\
\hline MM & 90.001 \\
\hline ML & 98.833 \\
\hline LH & 62.628 \\
\hline LM & 93.781 \\
\hline LL & 107.38 \\
\hline Average & $\mathbf{8 1 . 1 4 0}$ \\
\hline
\end{tabular}

All results shown up to this point (up to Figure 4.32) have been plotted as a function of time, where time is seen on the horizontal axis. This was done to have a consistent baseline in order to compare this research with previous research performed. This baseline (time on the horizontal axis) also allowed for an easier comparison of additional plots of speed, torque, power and efficiency as they changed over the course of each tests performed in this research.

\subsection{Efficiency vs. Output Speed}

The following plots, Figures 4.33 through 4.35, which show efficiency of the PD as a function of Output speed, may be used as a more relevant set of data when comparing the performance of the PD to other similar devices used in vehicle applications. 
Additionally, as can be seen from Figures 4.21-4.23 and 4.30-4.32, when the RPM of Output $\mathrm{C}$ would begin to increase, the efficiency of the PD would also begin to rise. This trend can be seen in many other geared mechanical devices including those in Figure 4.36, which shows Efficiency versus Speed of 4 different automotive transmissions [10]. Although the speeds of the additional 9 tests performed on the PD were limited by both the range of the electric motors and the design limitations of the PD, a similar trend to that seen in Figure 4.36 can also be seen in Figures 4.33 through 4.35 . Future testing would not be limited by slower speeds and would be expected to be closer to some of the higher efficiencies shown in Table 4.3. 


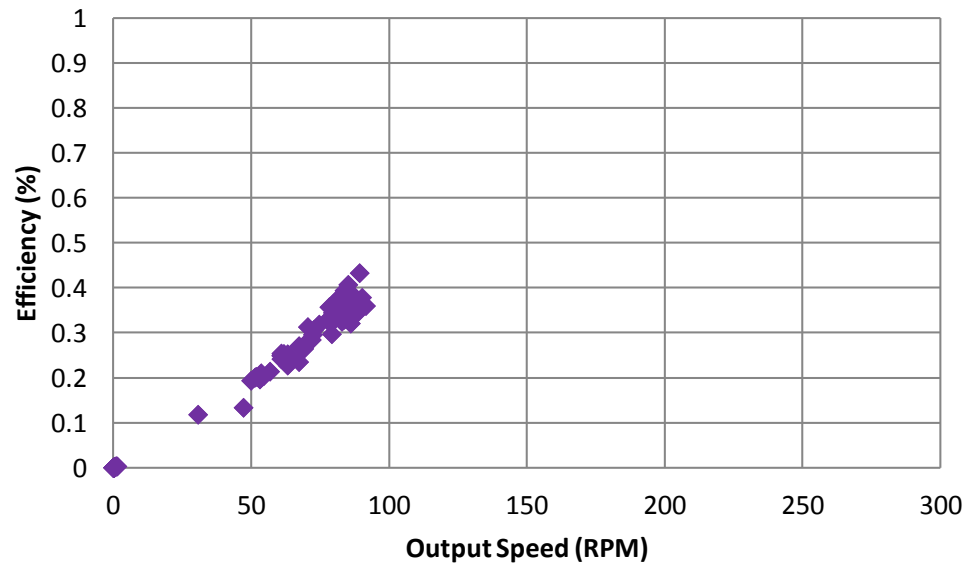

(a) $\mathrm{HH}$

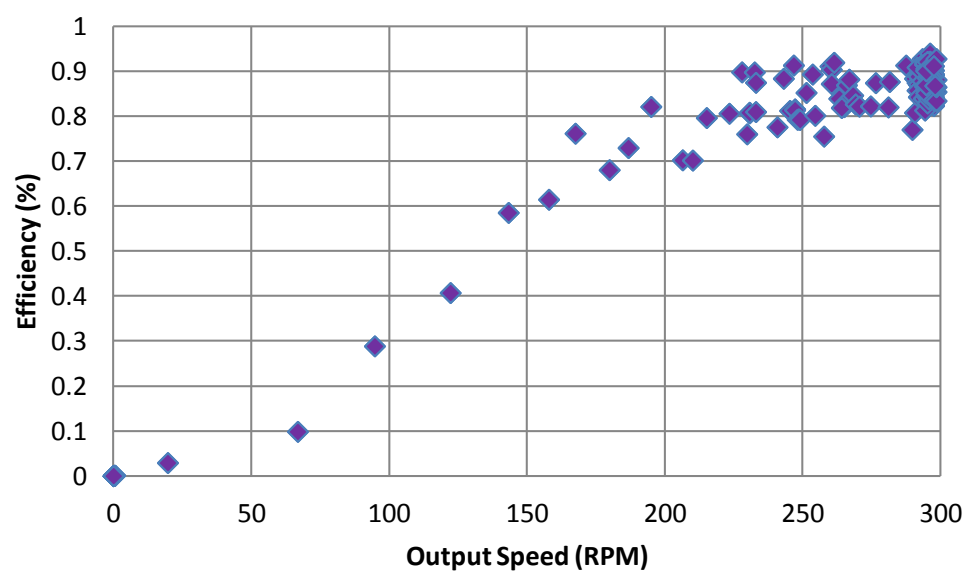

(b) $\mathrm{HM}$

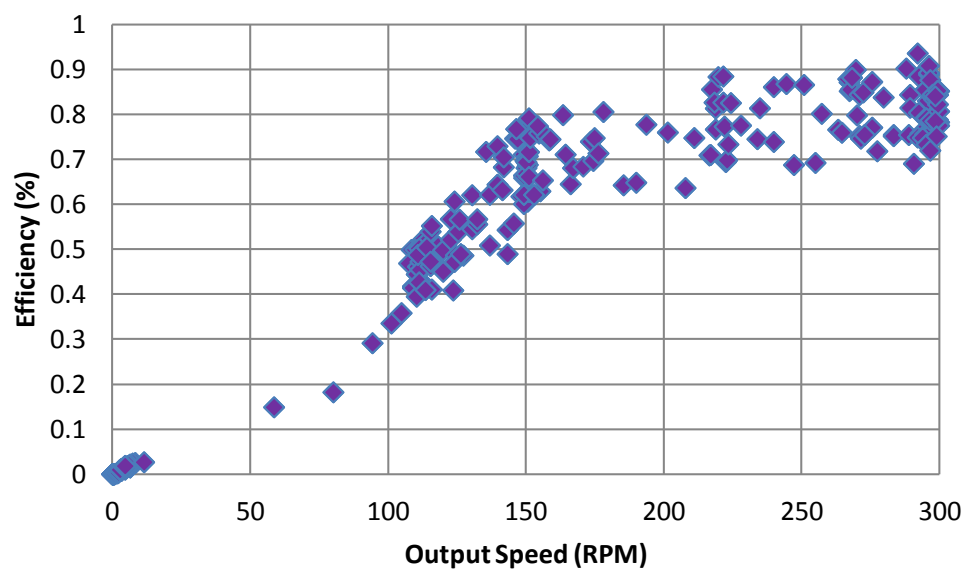

(c) $\mathrm{HL}$

Figure 4.33: Efficiency vs. Speed at High RPM with Varying Loads 


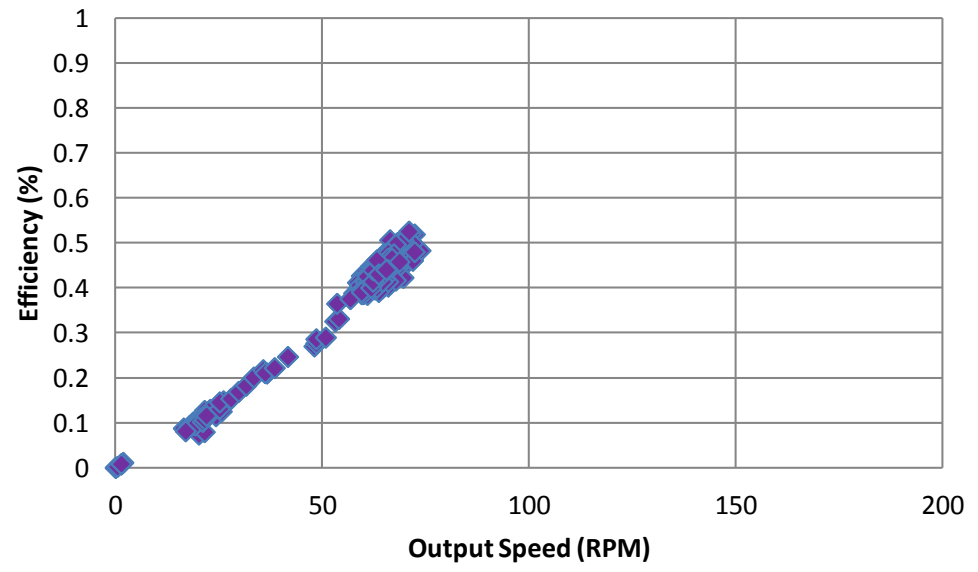

(a) $\mathrm{MH}$

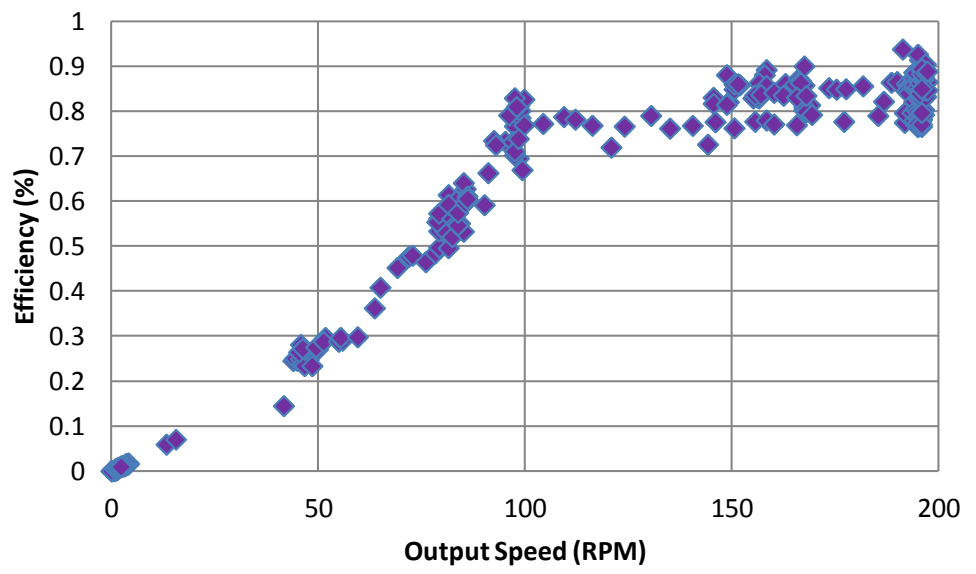

(b) $\mathrm{MM}$

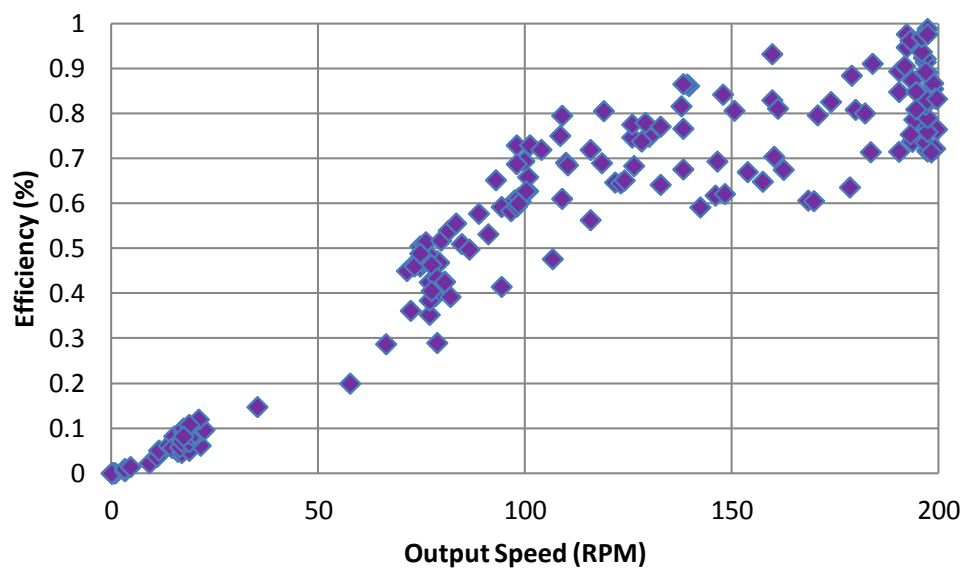

(c) ML

Figure 4.34: Efficiency vs. Speed at Medium RPM with Varying Loads 


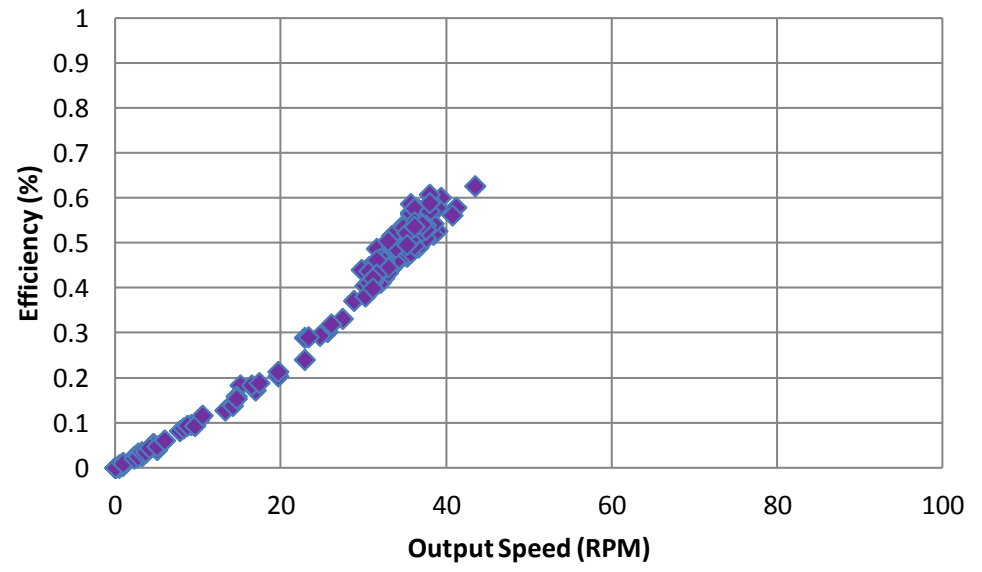

(a) $\mathrm{LH}$

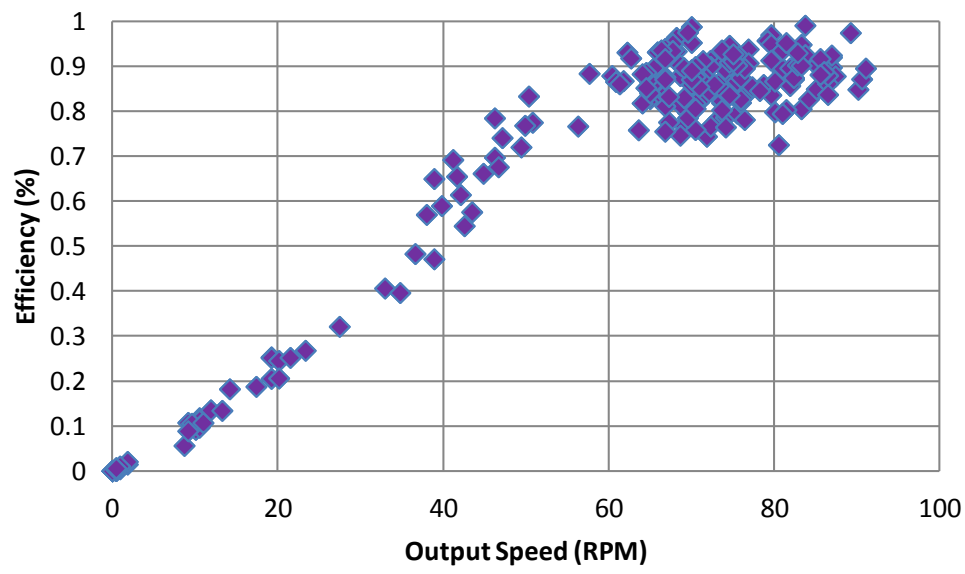

(b) LM

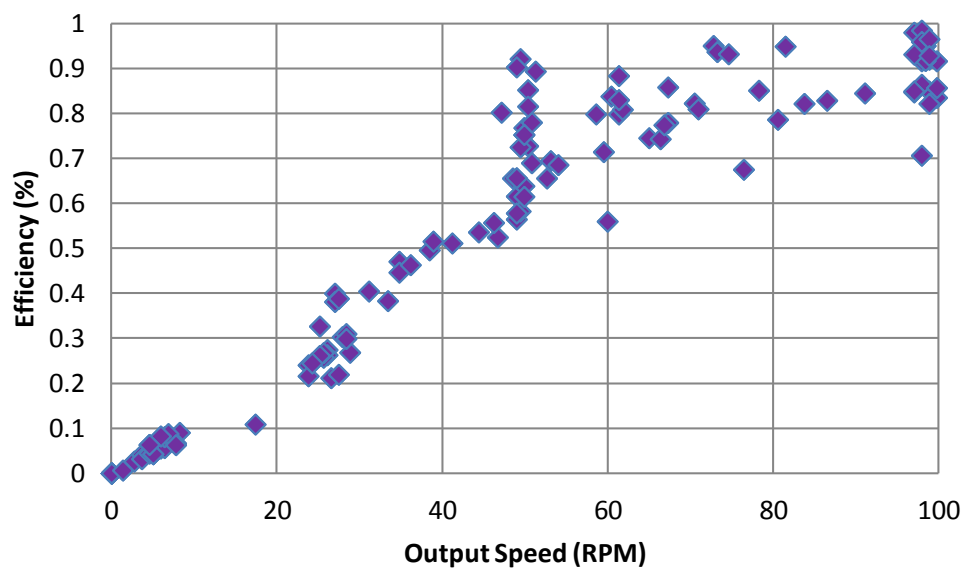

(c) LL

Figure 4.35: Efficiency vs. Speed at Low RPM with Varying Loads 


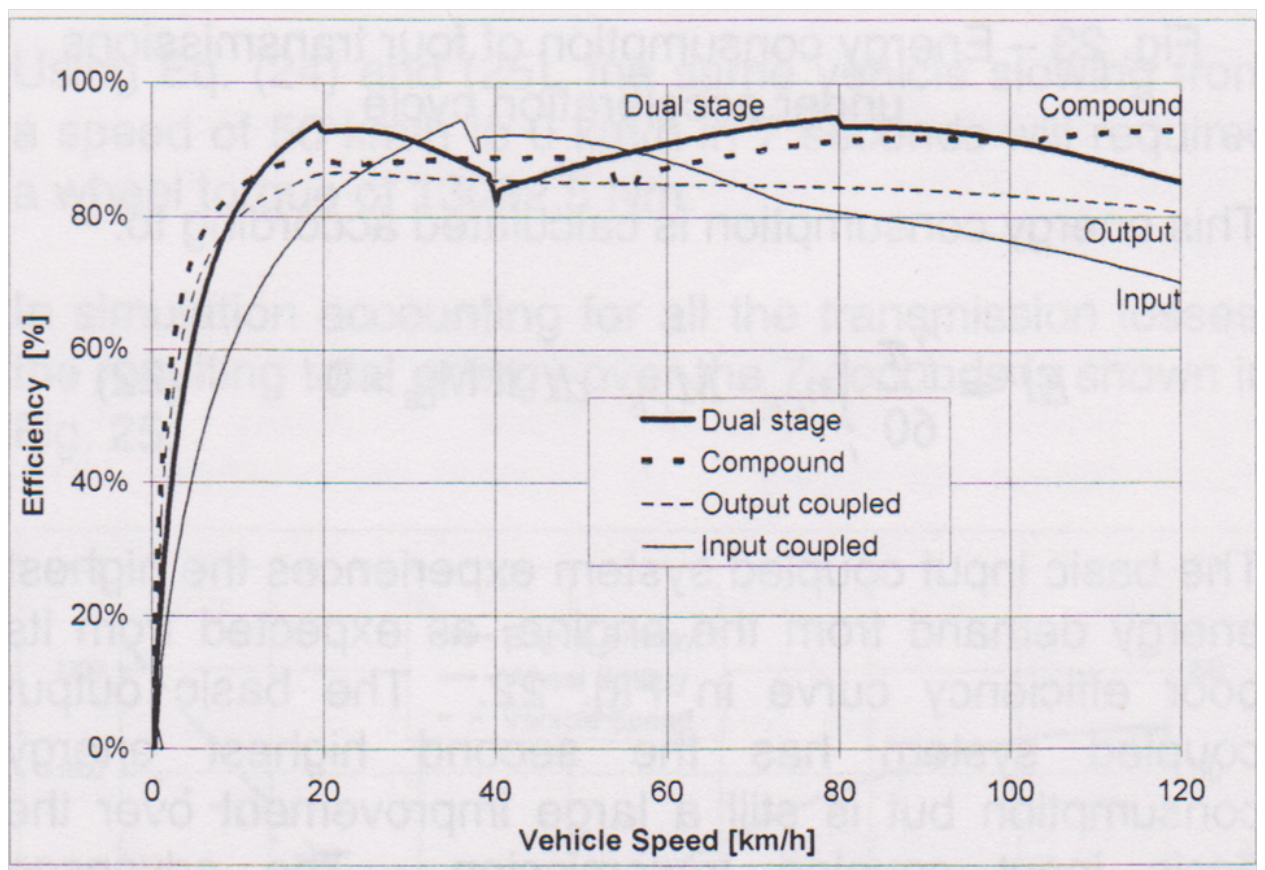

Figure 4.36: Efficiency vs. Speed of 4 Automobile Transmissions 


\subsection{Back-Drive Problem}

The next step in this research, to fully control the system and create the potential for it to replace a portion of the functionality of expensive electronic controllers, would be to investigate solutions to the back-drive problem. Wells proposed using a gear reduction system that allowed for enough reduction to overcome the back-drive issue [3]. The GTD and PD are torque splitting devices, as seen by the relationship inherent in Equation (4.2). If a gear reduction device with enough of a reduction to overcome the back-drive torque could be placed between Input B of the PD and the Control Motor (CM), then this could solve the back-drive problem.

The trade-off with adding a gear reduction device is the need for a Control Motor with a higher RPM range to match the RPM that is still needed at Input B of the PD. The advantage is that with the right gear reduction device, the PD would be able to function as a CVT without a back-drive issue. As was mentioned earlier, this problem is currently under investigation and will be examined and discussed in greater detail in future work. It can be stated, however, that the Planetary Differential has shown its potential as an appropriate $3 \mathrm{I} / \mathrm{O}$ system for use as a CVT in EV applications. 


\section{CHAPTER 5. CONCLUSION}

\subsection{How Research Objectives were Accomplished}

The main objective of this research was to more fully define the characteristics of a dualinput, single-output system that could achieve a higher efficiency than the previously tested GleasonTorsen Differential (GTD) and function as a Continuously Variable Transmission. The efficiencies obtained by testing the Planetary Differential (PD) were significantly higher than the GTD in all tests performed (see Tables 4.1 and 4.3) and are, therefore, considered to be sufficient in demonstrating the PD's potential as explained in this research (Chapter 4).

Tests performed in this work, along with previous research, were done with the intent of reducing the cost of EVs. It is anticipated that the cost of EVs could be reduced by reducing the cost of electronic controllers, which can be done by implementing the use of a Planetary Differential as has been discussed in this work, see Figure 5.3. A system such as the PD would provide EVs with a new architecture that would enable them to be less expensive, produce fewer emissions and additionally be safer during any maintenance that the vehicle may require.

Based on the mathematical modeling and tests that were performed in this work and the results that were obtained, the Planetary Differential, along with the architectural setup presented (again, see Figure 5.3) can be regarded as worth pursuing and/or investigating further in future

research work. To additionally demonstrate the value of this research and how the main objective was met, the following subsections explain how the initial 6 Objectives, as stated in Section 1.4, were accomplished.

\subsubsection{Objective 1}

Obtain a working knowledge (mathematical and physical) of various types of 3 Input/Output (I/O) systems, including their efficiencies. 
Objective 1 was accomplished through the study of various literature resources including textbooks, news articles and other journal articles which described the physical relationships of many types of mechanical, electrical and other similar systems. This research is evident in Chapter 2 of this work and specifically Section 2.5. Mathematical relationships of the system tested in this work (the Planetary Differential) were developed and verified as can be seen in Section 3.2 and Chapter 4 of this work.

After a study of many types of $3 \mathrm{I} / \mathrm{O}$ systems, it was concluded that the Planetary Differential was the most appropriate of those systems investigated in this work, see Table 2.2. It showed the greatest potential for the mechanical behavior that was required and additionally was the system recommended following previous work done on the GTD by Wells and Groen [3] [4].

\subsubsection{Objective 2}

Select and build (or have built) the appropriate type of 3 I/O system.

Objective 2 was accomplished by having a BYU-Idaho Capstone team of several students (and a faculty coach) complete the design of and build the Planetary Differential shown in Figure 3.1. The specifications for this type of system were provided and the team was responsible for purchasing, manufacturing and assembling the many components of the PD.

Although this PD demonstrated efficiency losses, these losses have been attributed to some minor imperfections that may have occurred in its construction and/or assembly. These efficiency losses would not be anticipated in a professionally manufactured Planetary Differential.

\subsubsection{Objective 3}

Test this system under various power combinations (i.e. vary the torque and speed of the different I/Os).

Objective 3 was accomplished throughout the various tests that were performed on the PD. The results from these test are displayed and explained in Chapter 4 of this work.

Not only were the same tests performed on the PD that were performed previously on the GTD, but additional relevant tests were also performed in an attempt to more effectively simulate real-life speed and loading scenarios. These additional tests can be seen in Section 4.2. 


\subsubsection{Objective 4}

Record the pertinent data during testing.

Objective 4 was very similar in procedure to Objective 3 and can also be seen in Chapter 4 of this work.

As has been mentioned earlier, torque and speed at each of the $3 \mathrm{I} / \mathrm{Os}$ of the PD were measured during all tests. To effectively calculate power at each of these I/Os, Equations (2.1) and (2.4) were used. Once power was obtained, Equation (2.11) was used to calculate efficiency for the system.

\subsubsection{Objective 5}

Analyze and compare the recorded data, including comparing it to the research performed previously.

Objective 5 was accomplished shortly after Objectives 3 and 4 and, again, can be seen throughout Chapter 4 of this work.

As is stated above, the main objective of this research was to more fully define the characteristics of a dual-input, single-output system that could achieve a higher efficiency than the previously tested Gleason-Torsen Differential. Table 4.1 shows a summary of efficiency data between the GTD and PD to demonstrate the PD's capability as a new potential solution. Table 4.3 shows a summary of efficiency data that was obtained through additional testing of the PD, which aid in demonstrating its capability.

\subsubsection{Objective 6}

Make recommendations for future research and innovation based on these findings.

The accomplishment of Objective 6 can be seen in the following recommendations for future work in testing the Planetary Differential.

- Investigate and implement a back-drive solution.

- Have a larger-scale PD professionally manufactured.

- Test new larger-scale PD. 


\section{Back-Drive Solution}

The next step in this research, as has been mentioned, would be to investigate and implement a solution to the back-drive problem. It is recommended that at least two different types of gear reduction systems, a worm gear and a series of spur gears, be connected to the PD and tested similar to tests performed in Section 4.2. The proposed setup can be seen in Figure 5.1, which is a modified version of Figure 3.5. Each of the back-drive preventing systems, as indicated by the light red box labeled "Back-drive System", would be placed between the Omega Torque Sensor and the Control Motor.

Performing the same or similar tests will allow the data collected from this work and future work to be easily compared and a potential back-drive solution to be easily recognized. By testing two different gear reduction systems, data from both systems could be compared and additional conclusions and/or recommendations could be inferred.

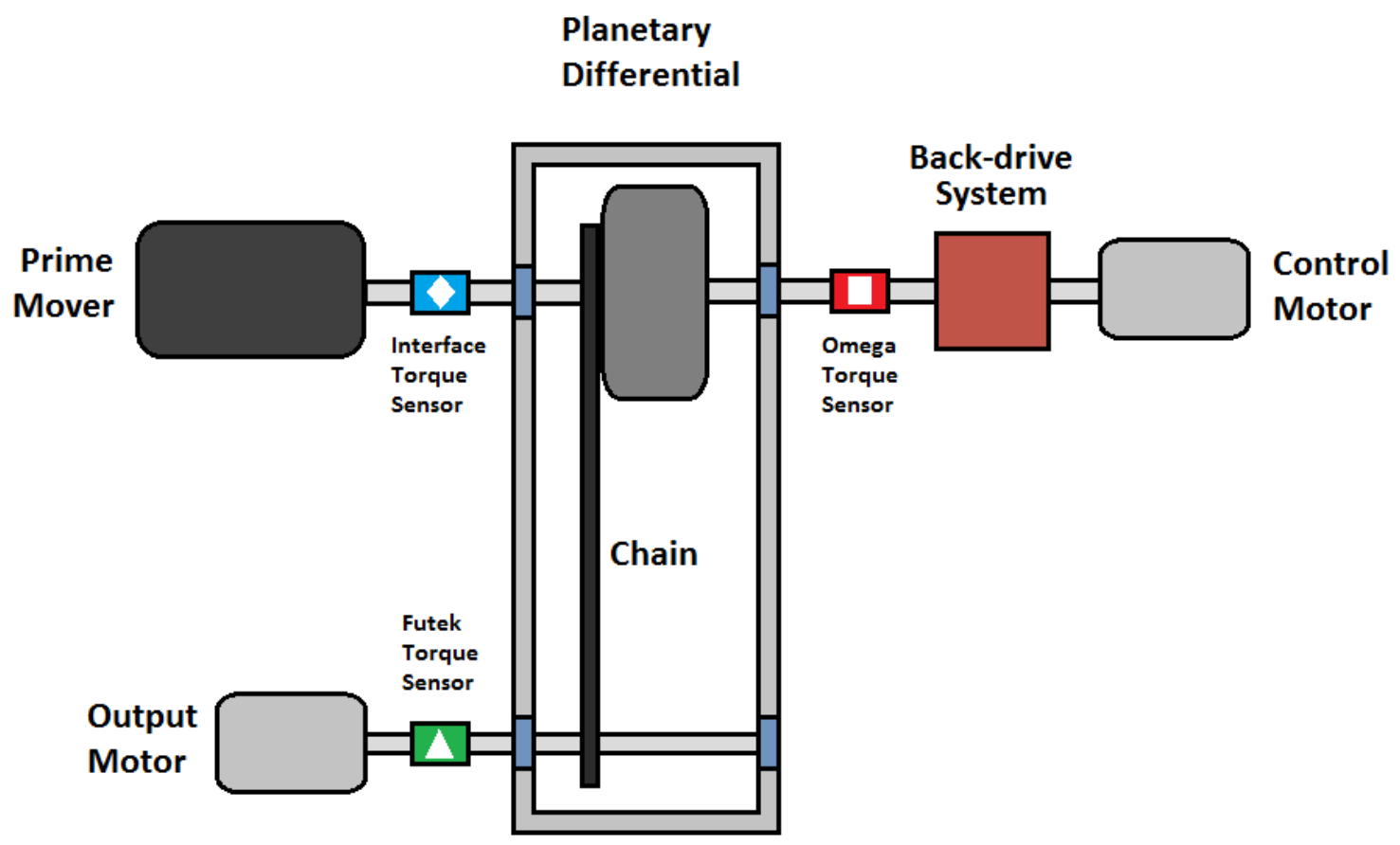

Figure 5.1: Experimental Setup with Back-drive Solution 


\section{Have a Larger-Scale PD Professionally Manufactured}

As was also mentioned previously in this work, the PD tested for this research was not entirely professionally manufactured/assembled. In order to more fully realize the PD's capability, a new PD should be professionally manufactured and assembled to eliminate the possibility of efficiency losses due to misalignment, improper meshing distance, etc.

Additionally, this new model of the PD should be a larger-scale model to allow for more realistic loads to be realized in its testing. It is assumed that with a larger and more expensive system built, tests could be performed at higher speeds allowing for more efficient results. It is also expected that much of the noise present in the data would be eliminated at higher speeds and higher loads.

\section{Test Larger-Scale PD}

More realistic or relevant loading conditions should be applied to this Larger-Scale PD by implementing it into an actual vehicle. The preference would be to test it in more than just an electric-only architecture (as was done in this research), but in a hybrid-electric architecture, as well. Figure 5.2, shown earlier in this work, demonstrates the electric-only setup that was investigated in this work and Figure 5.3 demonstrates the hybrid-electric setup that could be tested in future research. In these two figures, the Planetary Differential would be located in place of the box labeled "Transmission."

\subsection{A Final Word}

The recommendations outlined above for future research are the necessary steps to further the investigation that this research and previous research has accomplished. If these steps could be performed, it would contribute toward the potential of reducing the cost of electric and hybrid-electric vehicles, making them more affordable to the consumer, and safer when requiring maintenance.

With more affordable EVs capable of grasping a stronger hold on the automobile market, their cost would be expected to continue to decline, thus further accomplishing one of the goals of this research in producing high-mileage and low-emission vehicles at a more affordable price. 


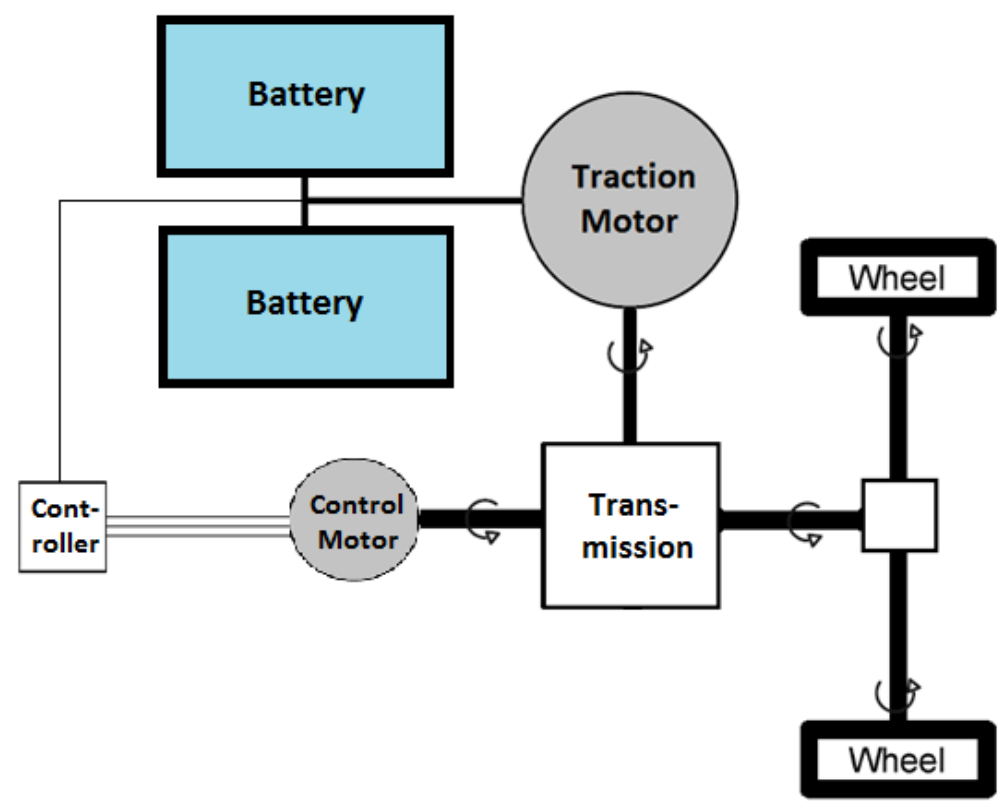

Figure 5.2: Electric-Only Powertrain Layout

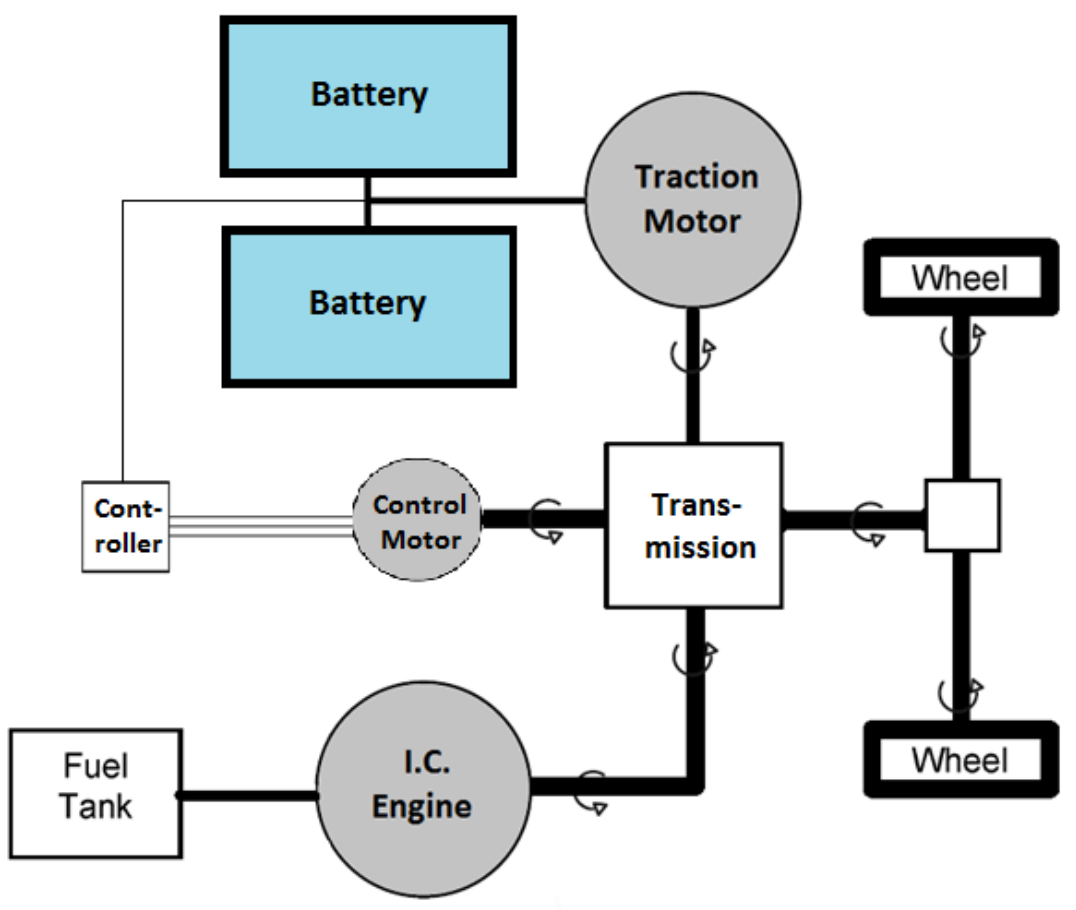

Figure 5.3: Hybrid-Electric Powertrain Layout 


\section{REFERENCES}

[1] de Han, R., 2012. Average U.S. Gasoline Price in January, February. 1

[2] Rizzoni, G., 2007. Principles and Applications of Electrical Engineering. McGraw-Hill. 3

[3] Wells, D. B., 2010. "Investigation of Mechanical Differentials as Continuously Variable Transmissions." Master's thesis, Brigham Young University. 3, 4, 5, 8, 26, 35, 37, 73, 99, 101

[4] Benjamin C. Groen, Dax B. Wells, R. H. T., 2011. "DC Motor Selection for Hybrid and Electric Vehicles using and Infinitely Variable Transmission." In International Design Engineering Technical Conference. 3, 4, 8, 26, 35, 101

[5] Automobile Drivetrain History http://www.motorera.com/history/hist10.htm. 7

[6] The Model $\mathrm{T}$ Put the World on Wheels http://corporate.ford.com/aboutford/heritage/vehicles/modelt/672-model-t. 7

[7] Frank A. Fitz, P. B. P., 1991. "A geared infinitely variable transmission for automotive applications." In SAE Technical Paper Series, SAE International, SAE International. 8

[8] Kluger, M. A., and Long, D. M., 1999. An overview of current automatic, manual and continuously variable transmission efficiencies and their projected future improvements Tech. Rep. 1999-01-1259, SAE International, Warrendale, PA, Mar. 8, 11, 12

[9] Ai, X., and Anderson, S., 2005. An Electro-Mechanical infinitely variable transmission for hybrid electric vehicles Tech. Rep. 2005-01-0281, SAE International, Warrendale, PA, Apr. 8

[10] Carl, B., Ivantysynova, M., and Williams, K., 2006. Comparison of operational characteristics in power split continuously variable transmissions Tech. Rep. 2006-01-3468, SAE International, Warrendale, PA, Oct. 8, 94

[11] Hosokawa, R. S., Stevaux, R., and Martins, A., 2011. Lightweight differential Tech. Rep. 2011-36-0105, SAE International, Warrendale, PA, Oct. 8

[12] Wikipedia contributors, 2012. Horsepower, June Page Version ID: 495510329. 9

[13] Colin R. Ferguson, A. T. K., 2001. Internal Combustion Engines: Applied Thermosciences., 2nd ed. John Wiley \& Sons, Inc. 9

[14] Gisbert Lechner, H. N., 1999. Automotive Transmissions: Fundamentals, Selection, Design and Application. Springer-Verlag. 10, 20 
[15] Wikipedia contributors, 2012. Hydramatic, May Page Version ID: 493270343. 10

[16] J. "Kelly" Flory, J., 2008. American Cars, 1946-1959: Every Model, Year by Year. McFarland \& Coy. 10

[17] Amendola, C. H. F., and Alves, M. A. L., 2006. Gear shift strategies analysis of the automatic transmission in comparison with the double clutch transmission Tech. Rep. 2006-01-2872, SAE International, Warrendale, PA, Nov. 11

[18] Helms, R., 2006. Shifting over to CVT, January. 12

[19] Farzad Samie, B. G., 2005. "Comparison of efficiency measurements and simulation results for automotive traction drives." SAE International. 13

[20] Tomoyuki, H., Hiroshi, I., Hiraku, O., Shinobu, K., and Kazuhiro, T., 2005. "A study of the power transfer systems for HEV." Transaction of Society of Automotive Engineers of Japan, 36(5), pp. 137-143. 14

[21] W. Kramer, S. Chakraborty, B. K., and Thomas, H., 2008. Advanced power electronic interfaces for distributed energy systems, March. 14

[22] Company, T. M., 2011. Hybrid 2012 Model Emergency Response Guide. 16

[23] John Cadick, Mary Capelli-Schellpfeffer, D. K. N. A. W., 2012. Electrical Safety Handbook. McGraw-Hill Companies, Inc. 16

[24] Muller, H. W., 1982. Epicyclic Drive Trains: Analysis, Synthesis, and Applications. Wayne State University Press. 16, 25, 26, 28, 33

[25] Lanka, Suhan Dharmasuriya, H.-S., 2010. Suhan-auto: Locking differential, Oct. 17

[26] The Strange Geometry of Gleason's Impossible Differential. 18

[27] Norton, R. L., 2008. Design of Machinery., 4 ed. McGraw-Hill Companies, Inc. 20, 26, 28, $44,45,46$

[28] Dax B. Wells, Benjamin C. Groen, R. H. T., 2011. "Investigation of Mechanical Differentials as Continuously Variable Transmissions." In International Design Engineering Technical Conference, ASME, ASME. 20, 26

[29] Wikipedia contributors, 2012. Epicyclic gearing, May Page Version ID: 490005239. 20

[30] Christensen, M., 2002. "Analysis of Pivot-Arm Continuously Variable Transmissions.” Master's thesis, Brigham Young University. 21

[31] Andersen, B. S., 2007. "An Investigation of a Positive Engagement, Continuously Variable Transmission.” Master's thesis, Brigham Young University. 22

[32] Telford, C., 2012. "An Investigation of the Ward Leonard System for use in a Hybrid or Electric Passenger Vehicle.” Master's thesis, Brigham Young University. 24 
APPENDIX A. CAD DRAWINGS 
PRODUCED BY AN AUTODESK EDUCATIONAL PRODUCT

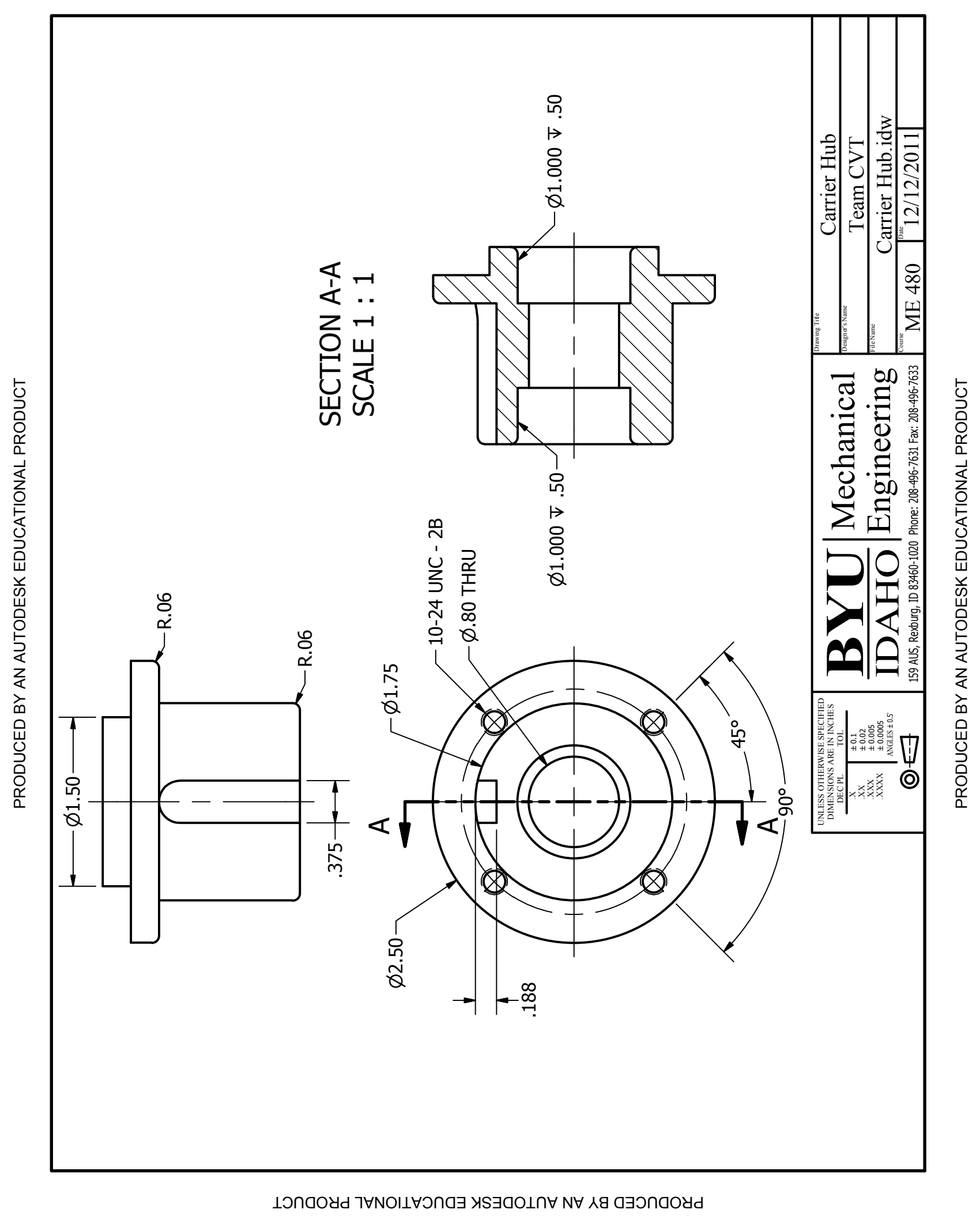




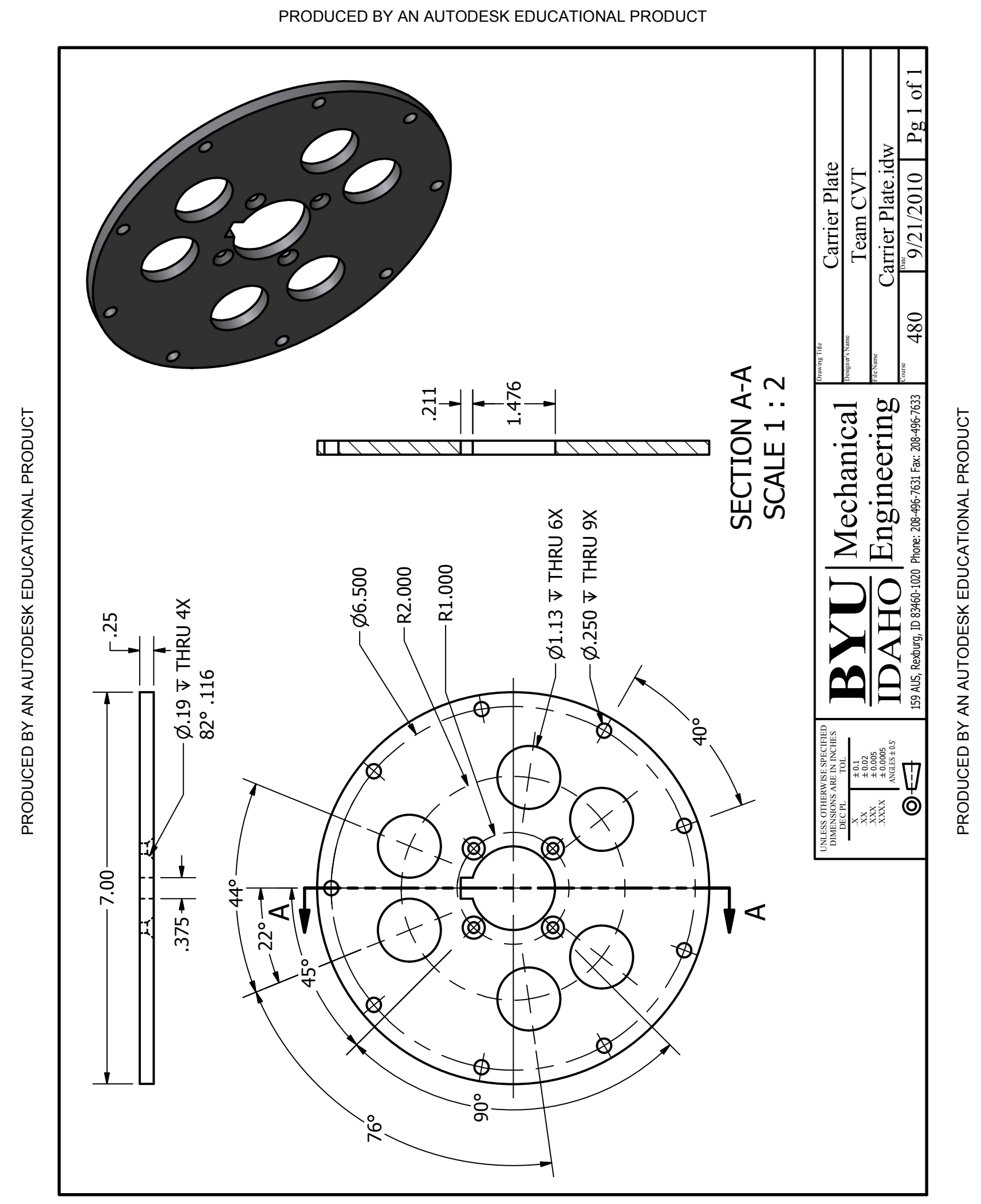

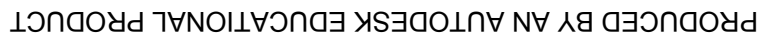




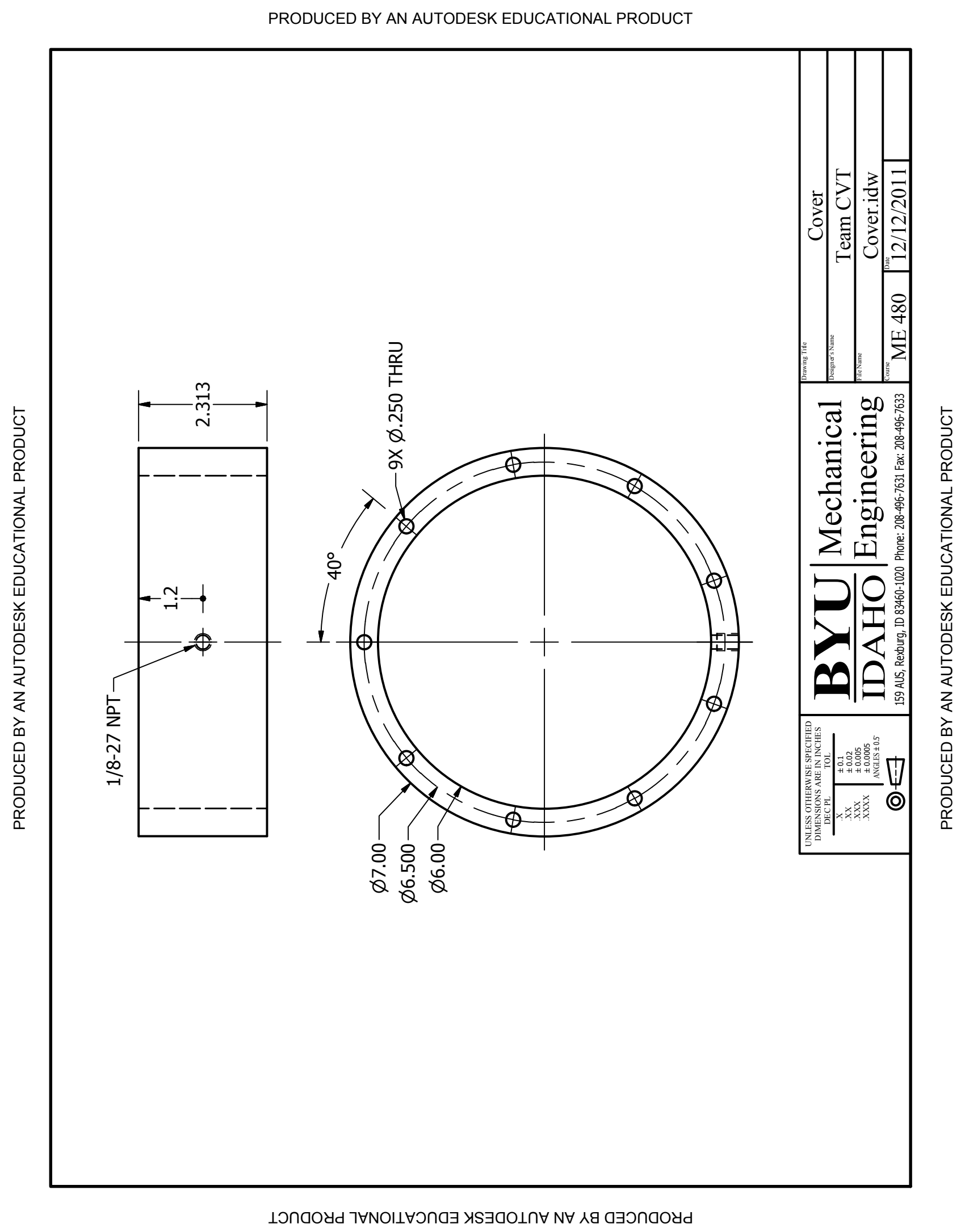


PRODUCED BY AN AUTODESK EDUCATIONAL PRODUCT

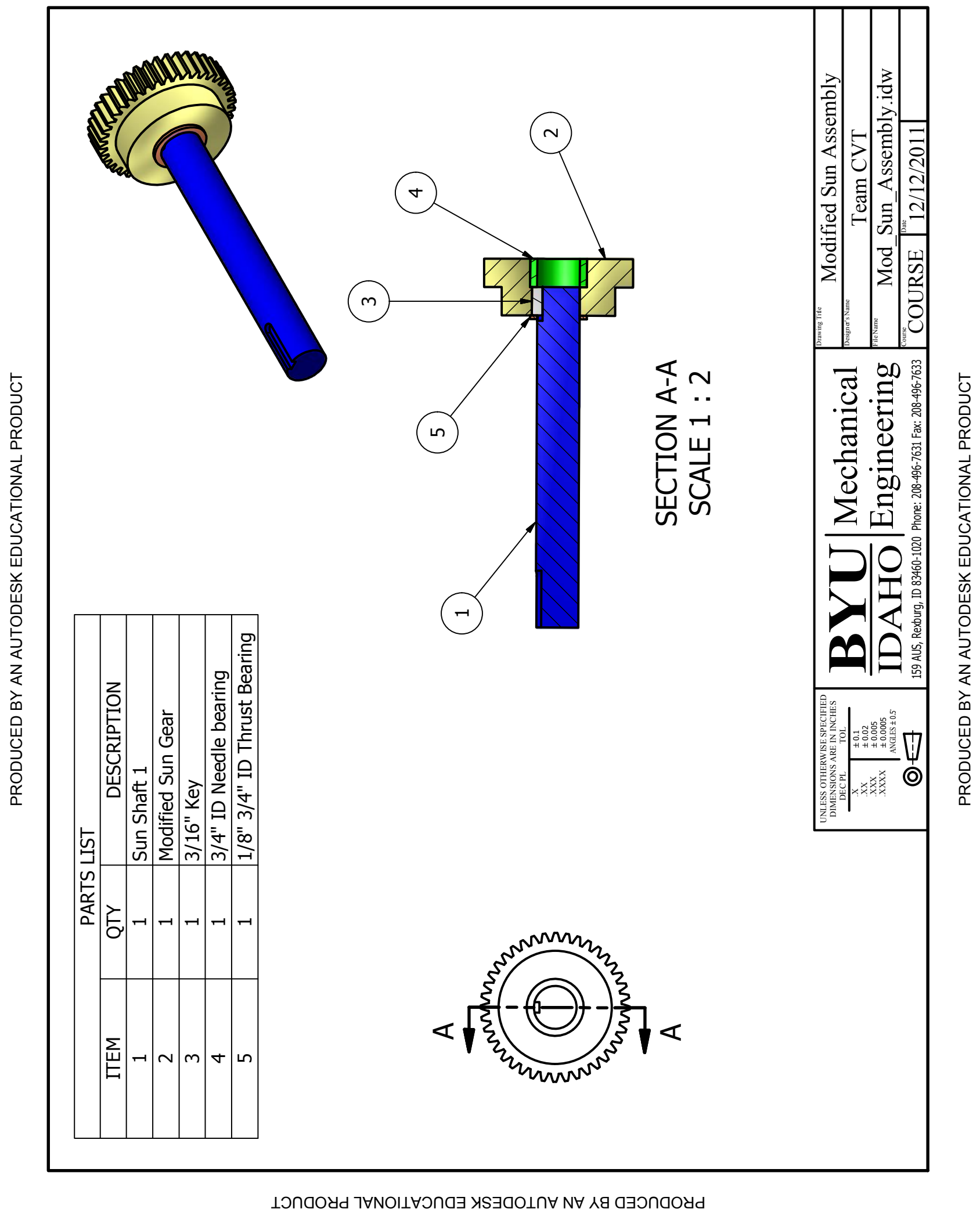


PRODUCED BY AN AUTODESK EDUCATIONAL PRODUCT

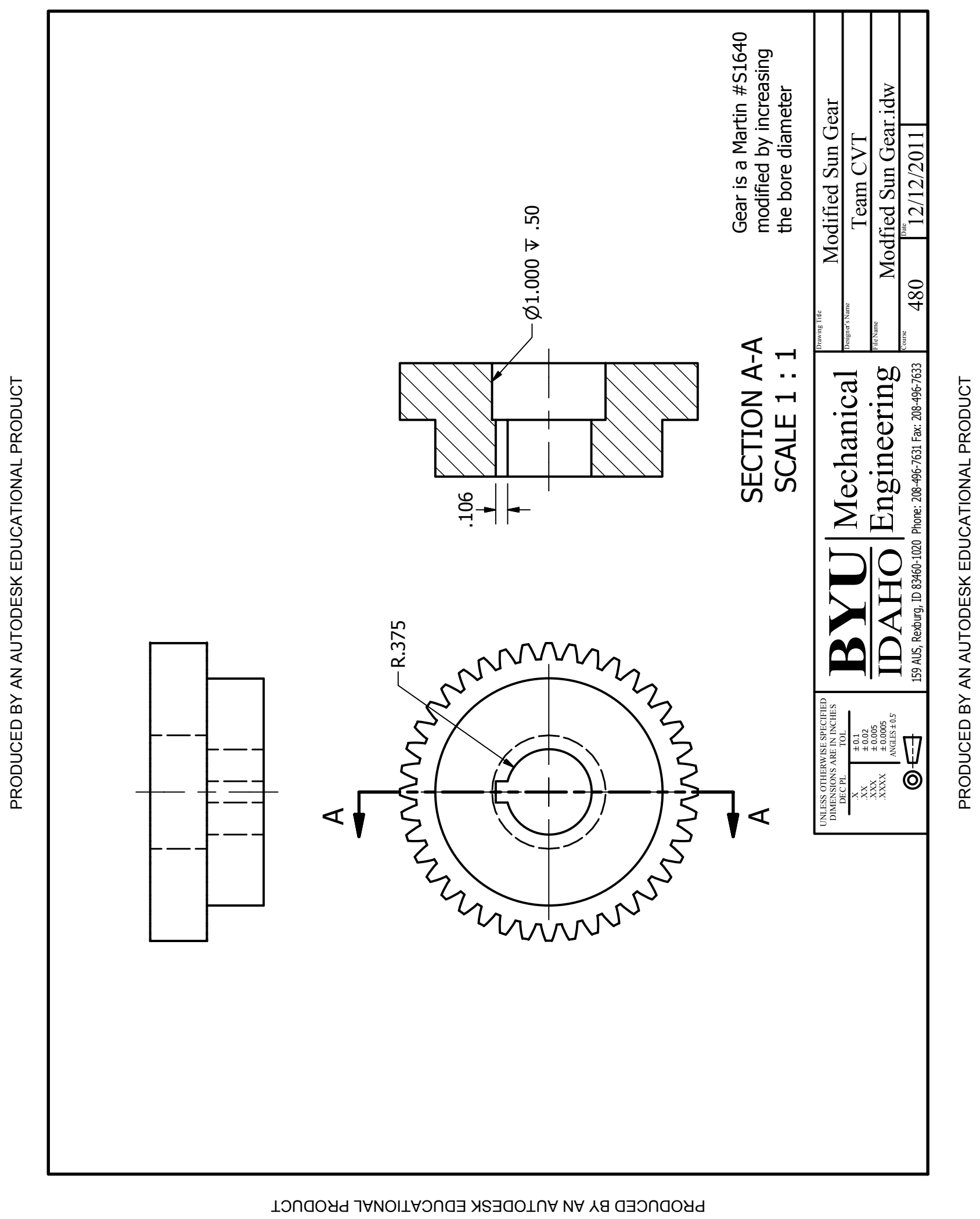


PRODUCED BY AN AUTODESK EDUCATIONAL PRODUCT

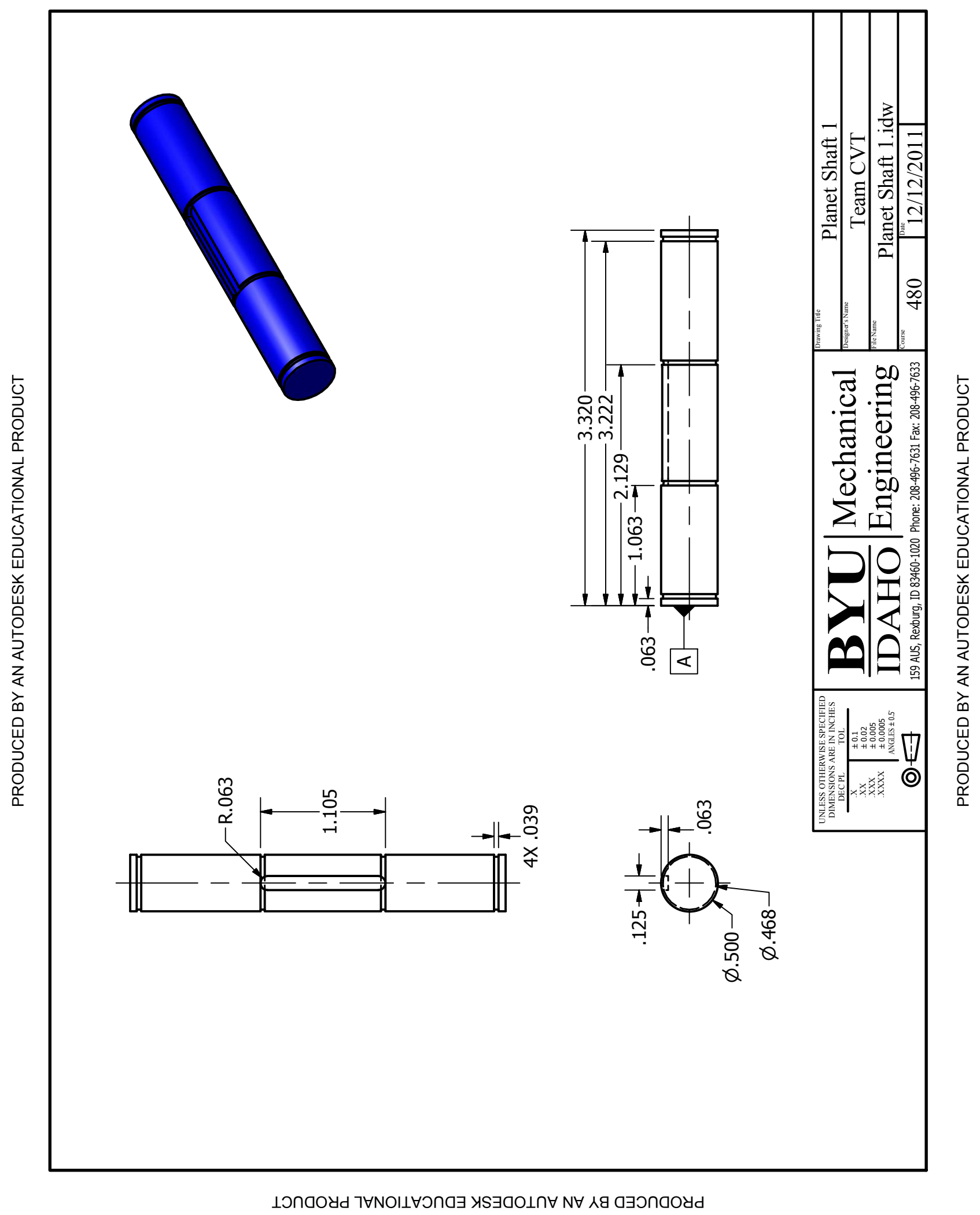


PRODUCED BY AN AUTODESK EDUCATIONAL PRODUCT

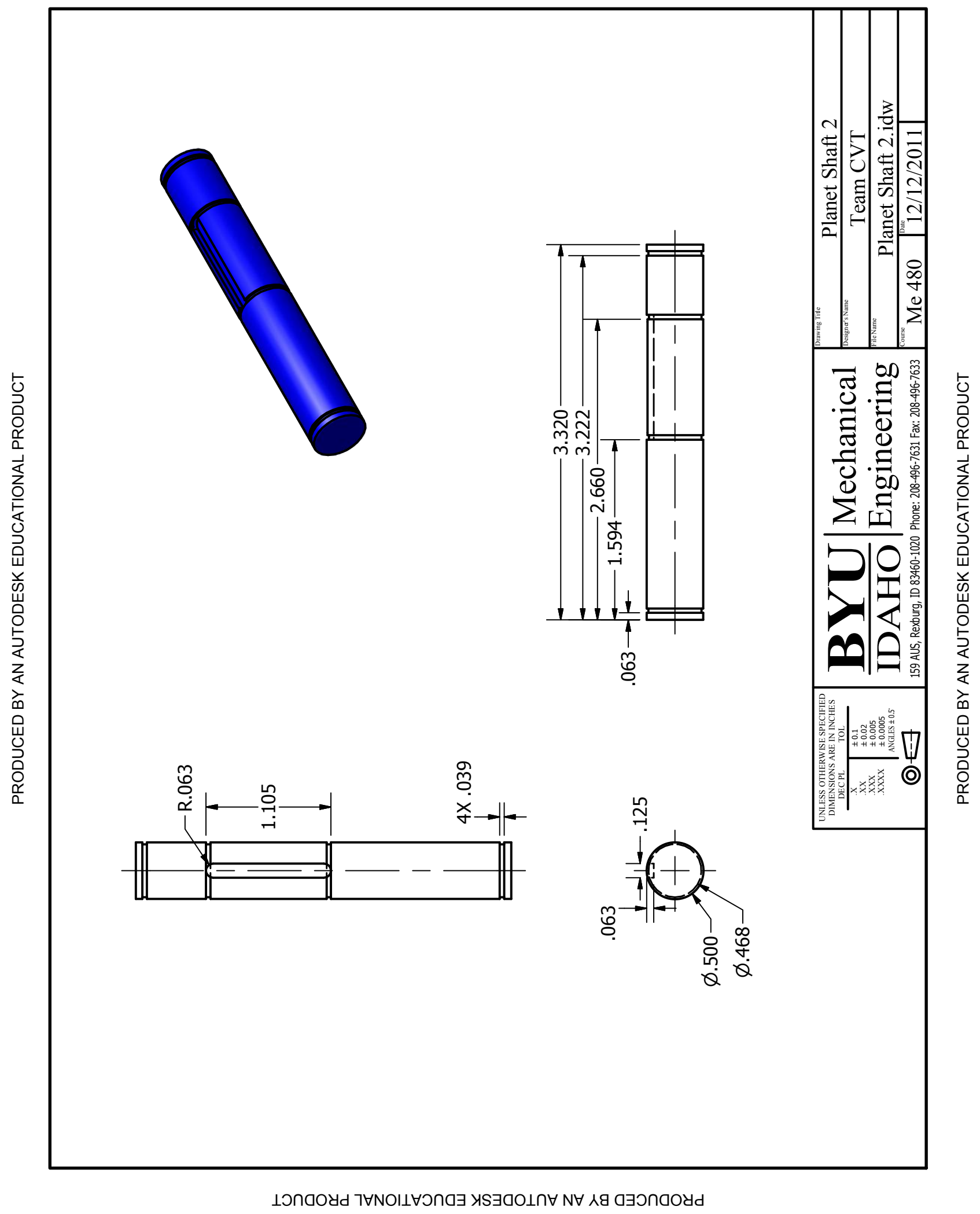


PRODUCED BY AN AUTODESK EDUCATIONAL PRODUCT

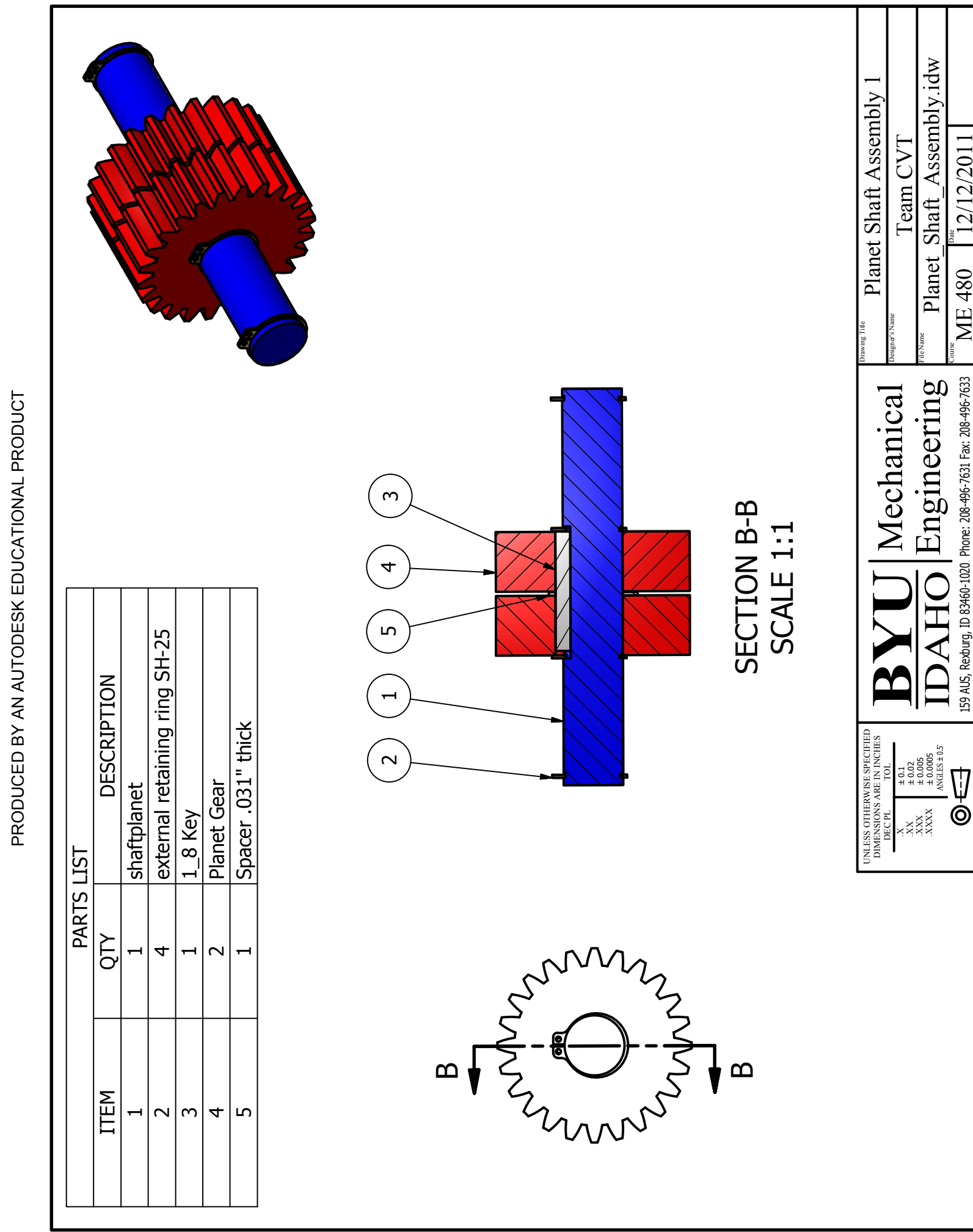

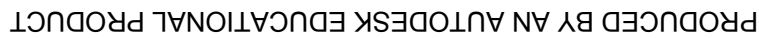


PRODUCED BY AN AUTODESK EDUCATIONAL PRODUCT

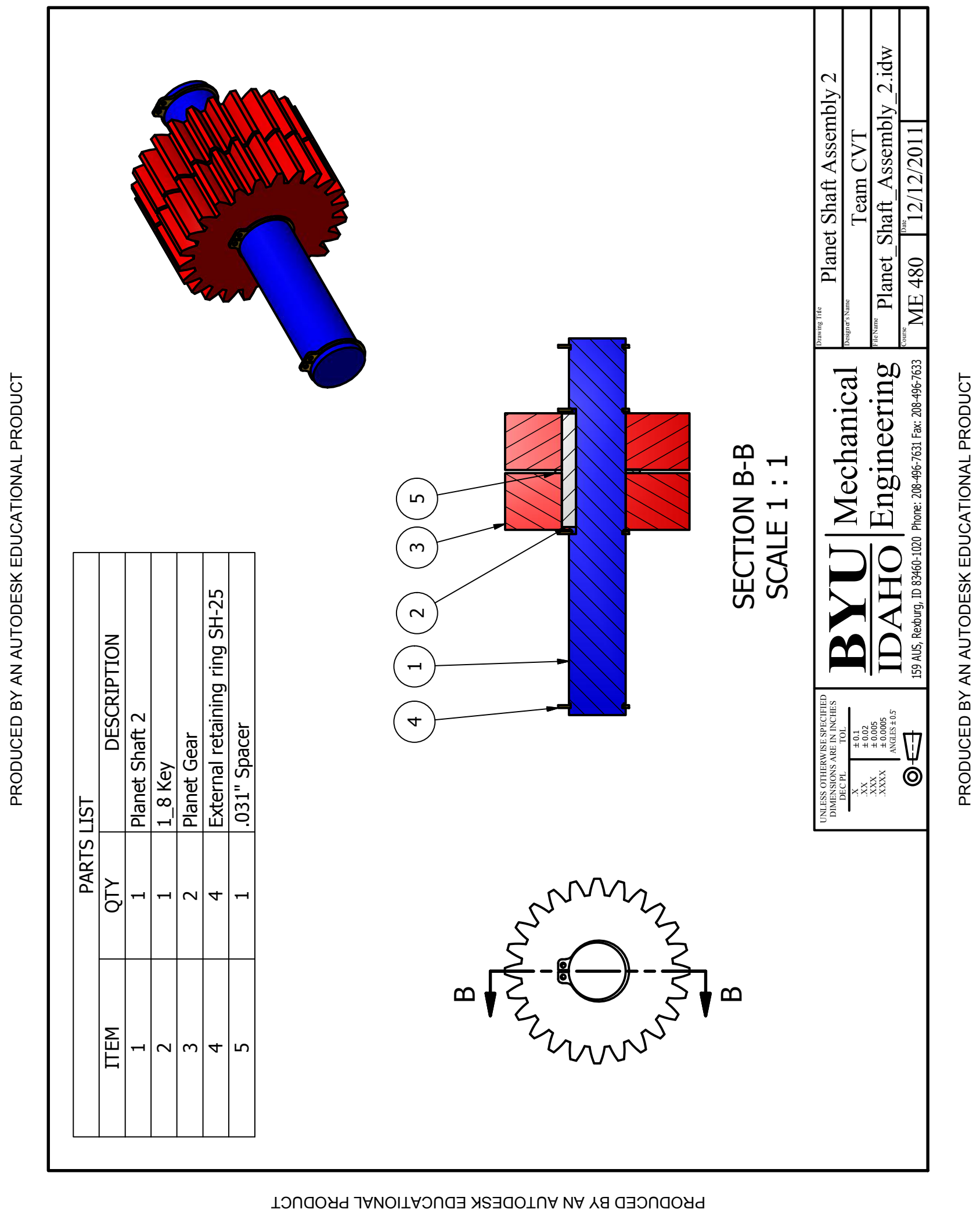




\section{PRODUCED BY AN AUTODESK EDUCATIONAL PRODUCT}

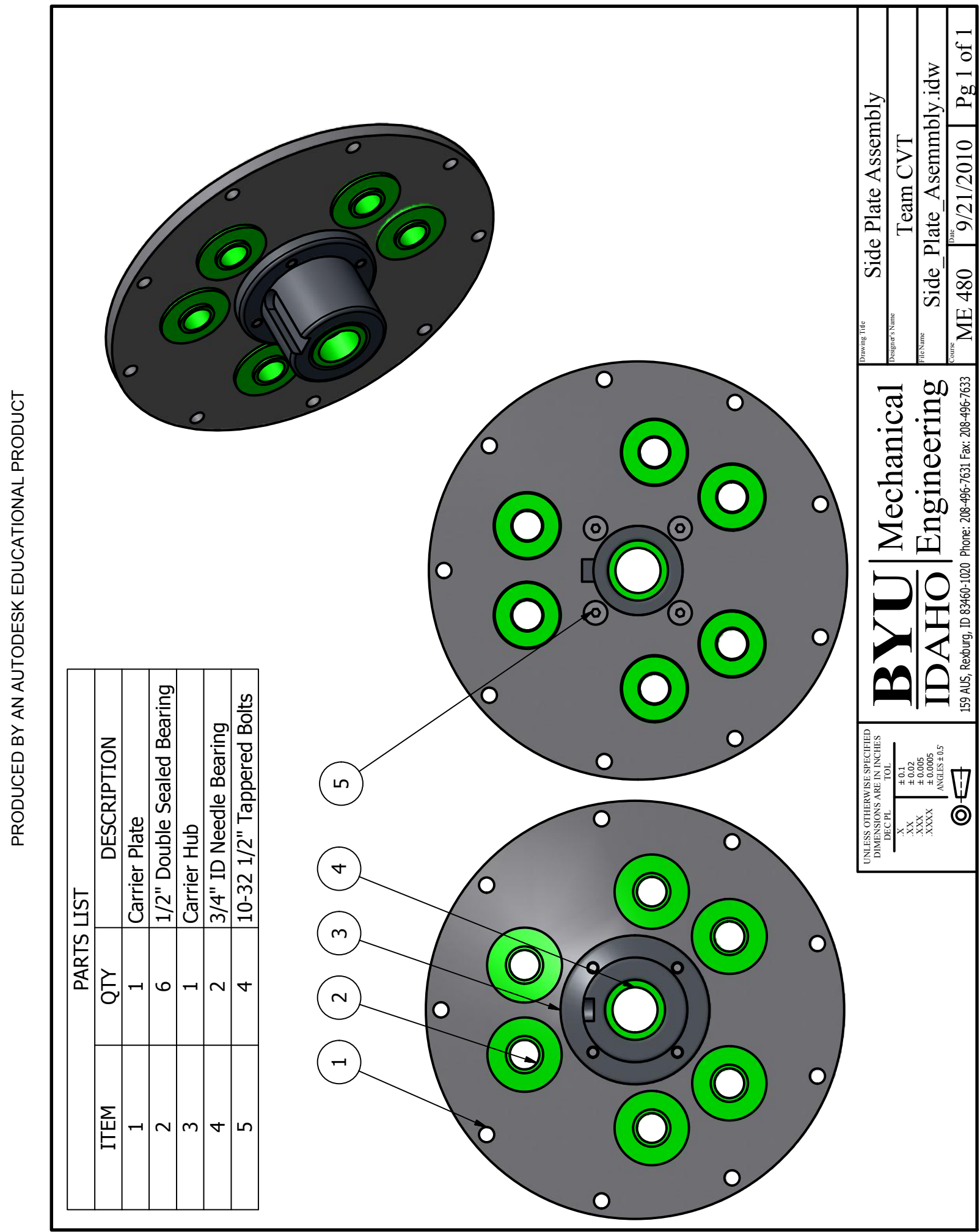

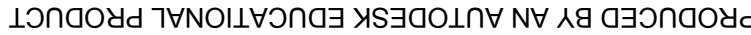


PRODUCED BY AN AUTODESK EDUCATIONAL PRODUCT

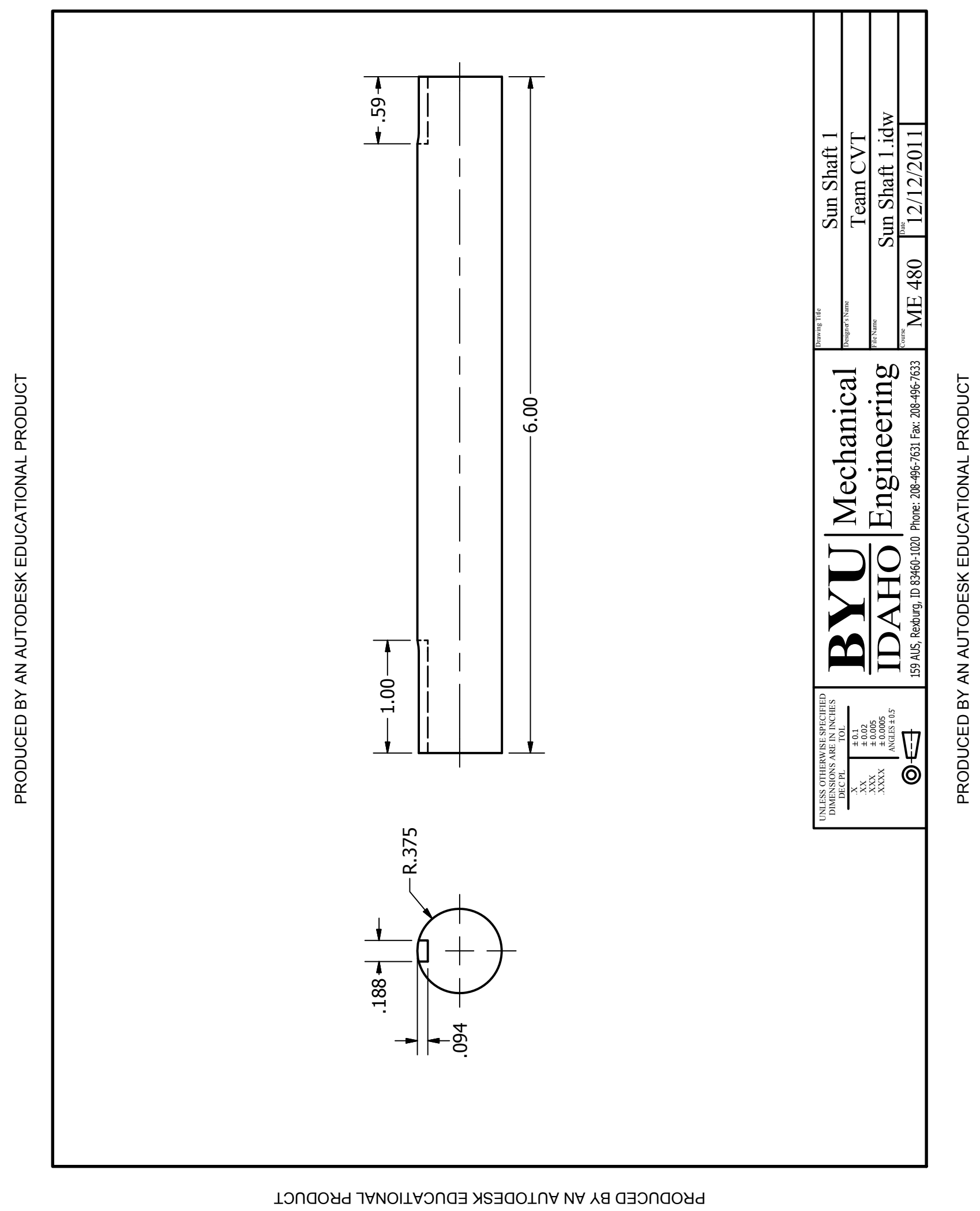


PRODUCED BY AN AUTODESK EDUCATIONAL PRODUCT

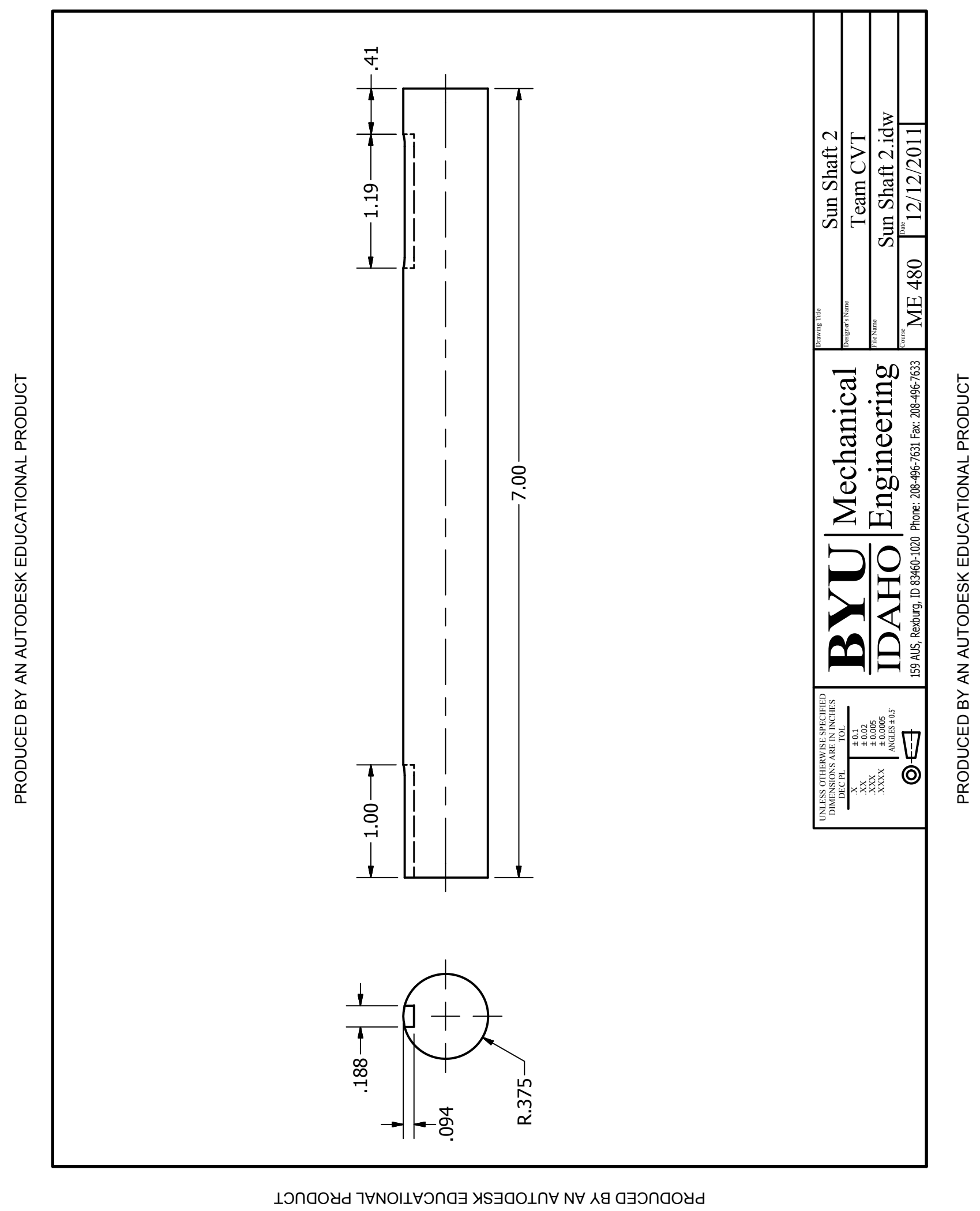


PRODUCED BY AN AUTODESK EDUCATIONAL PRODUCT

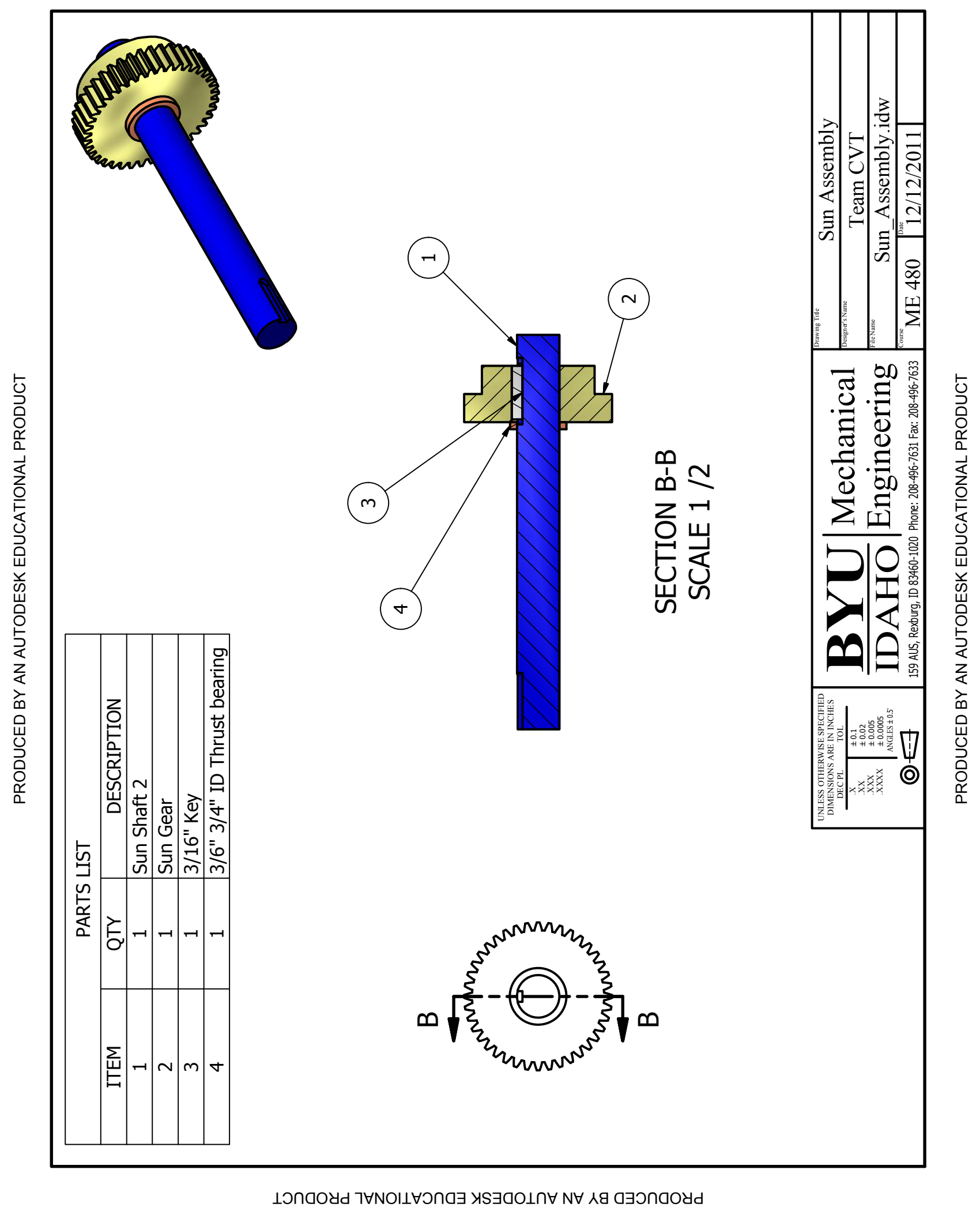


PRODUCED BY AN AUTODESK EDUCATIONAL PRODUCT

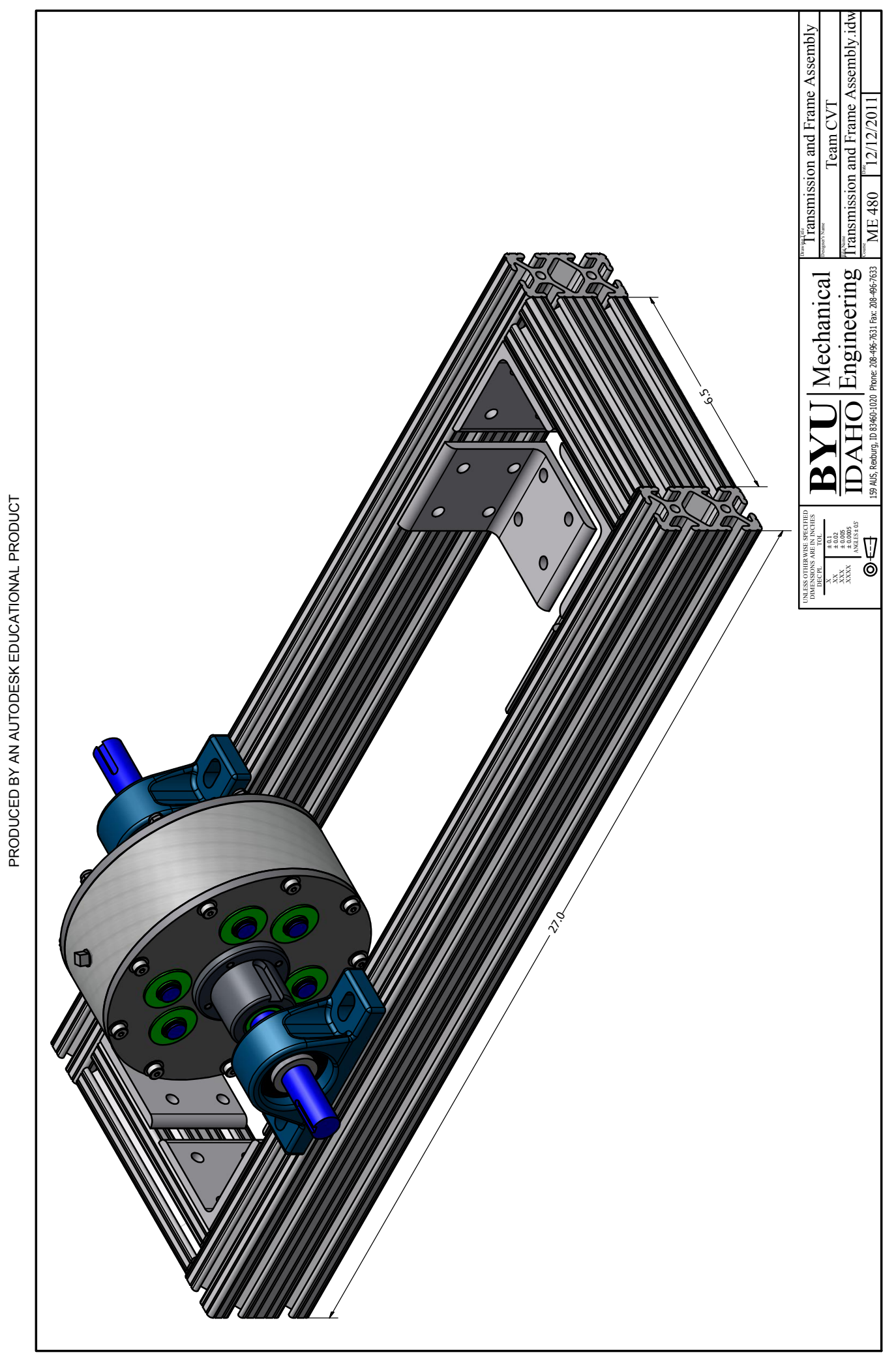

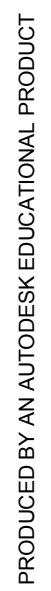

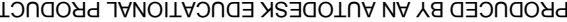




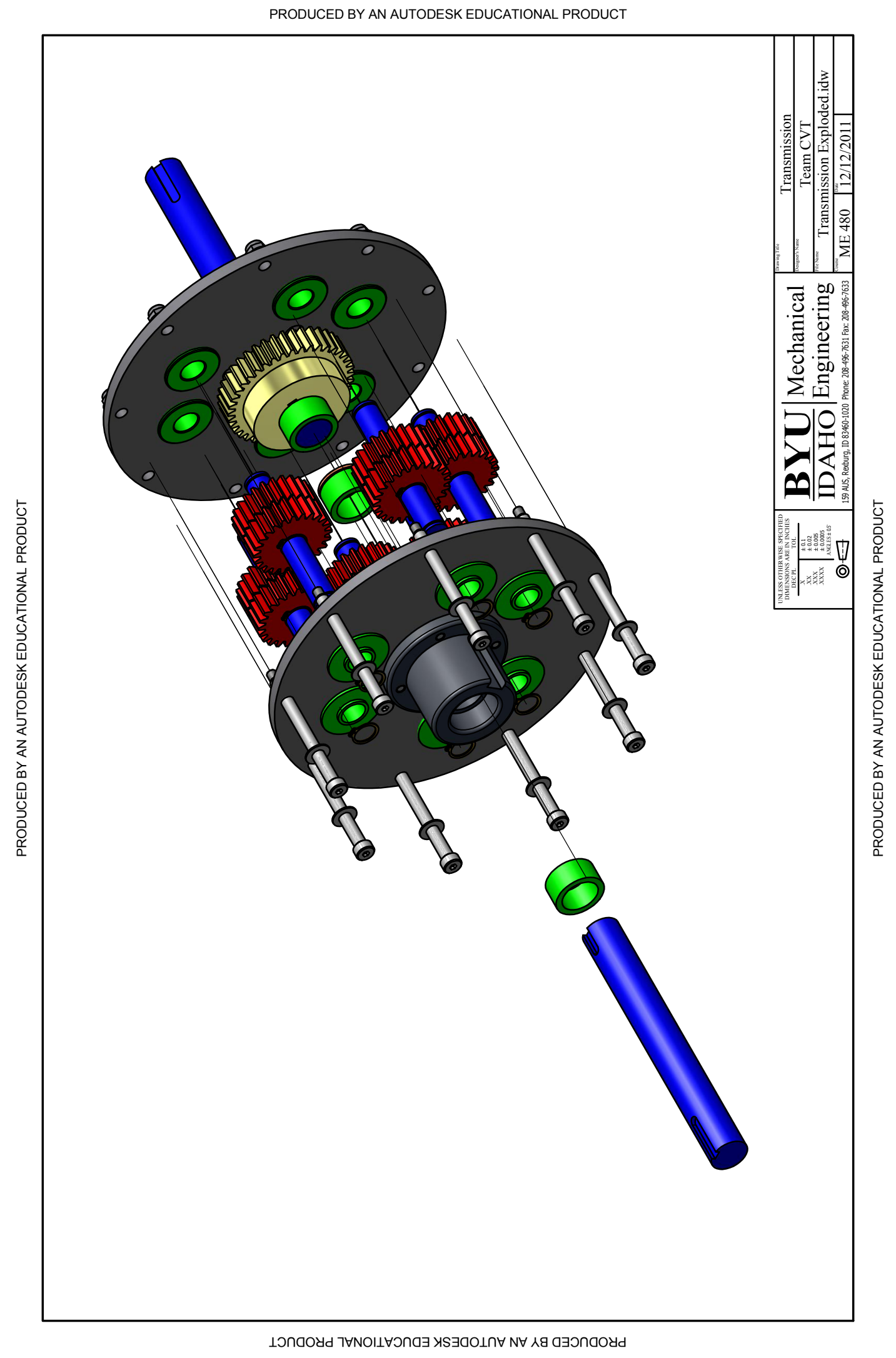




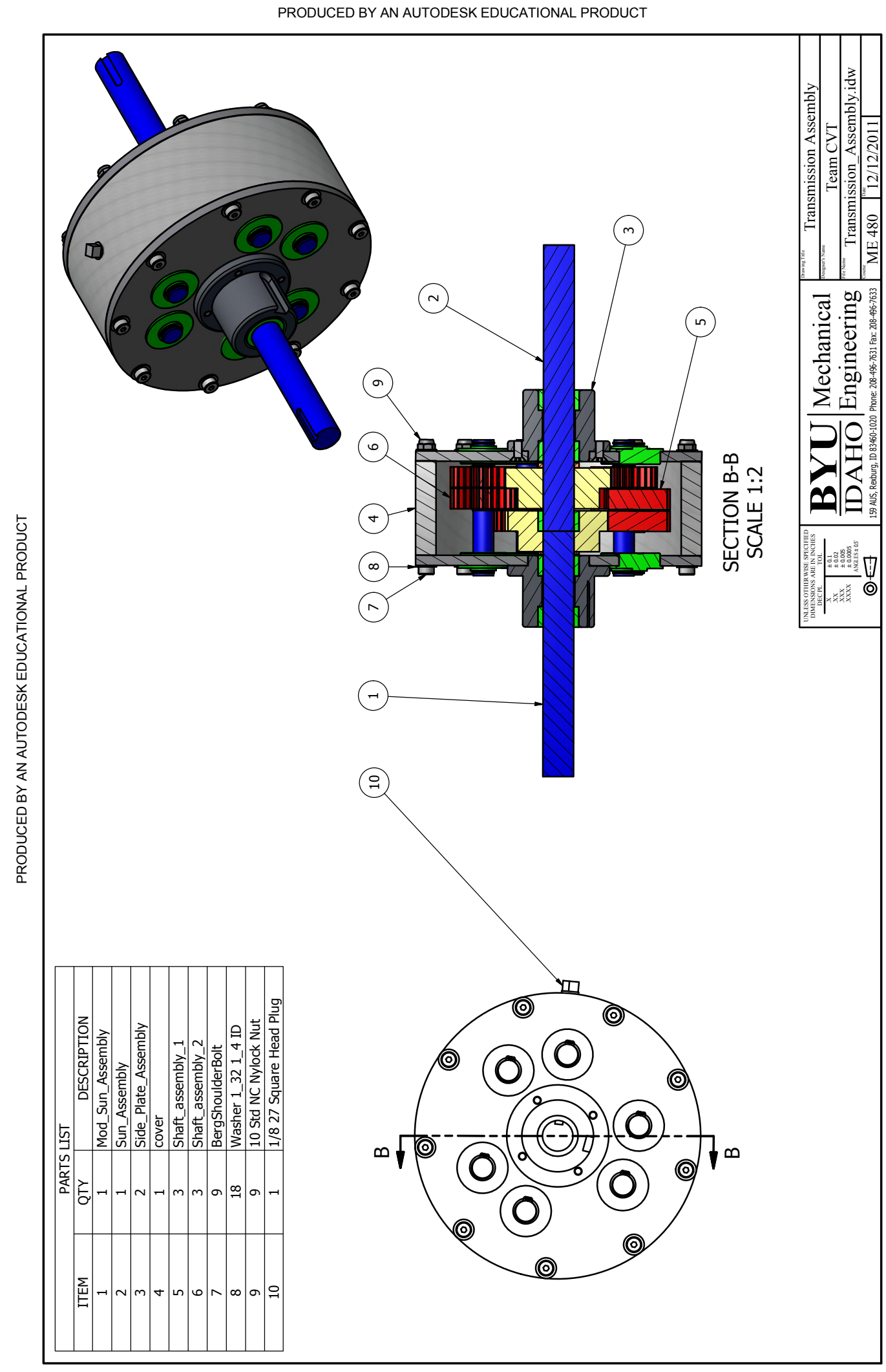

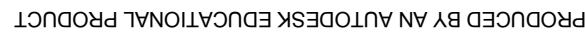




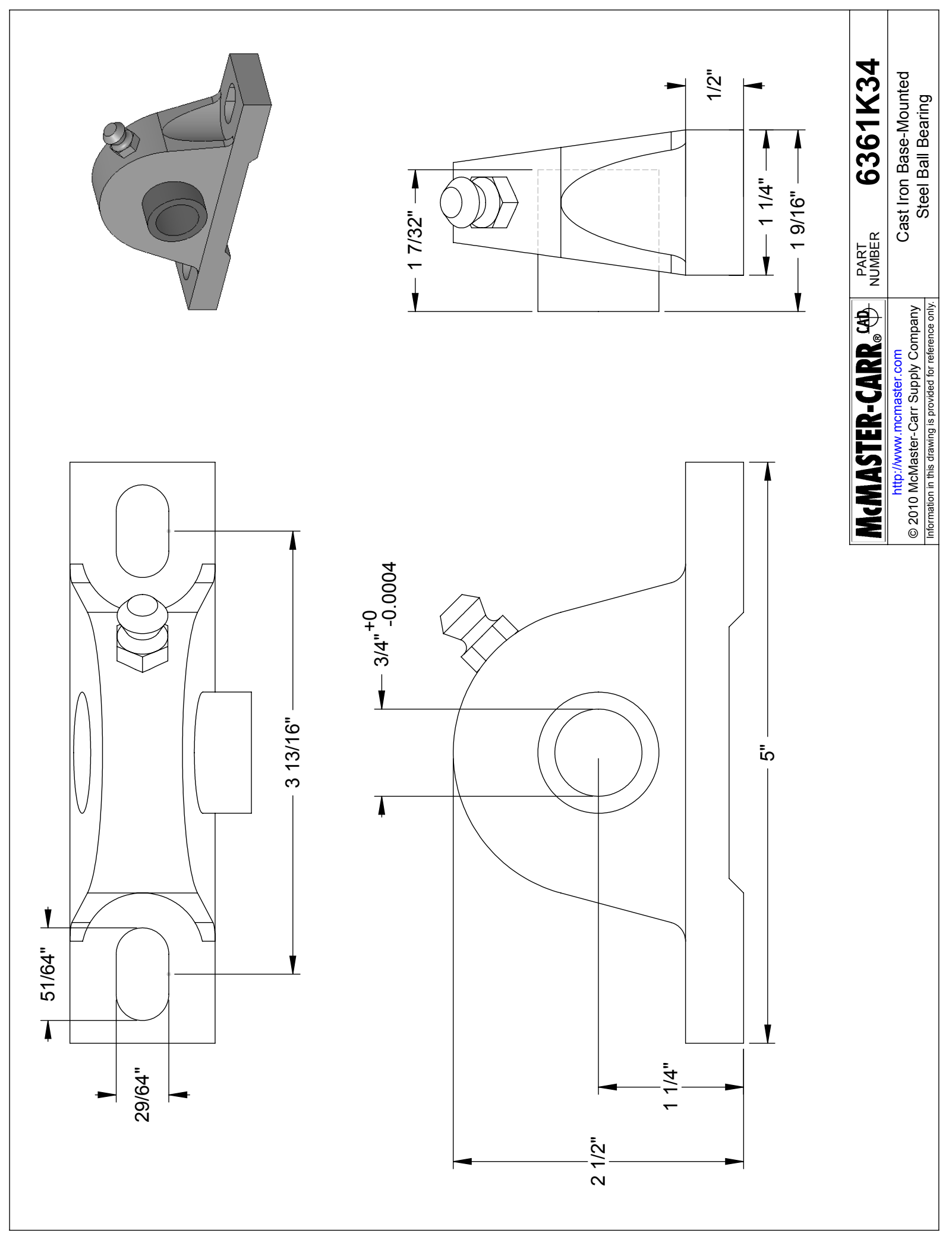




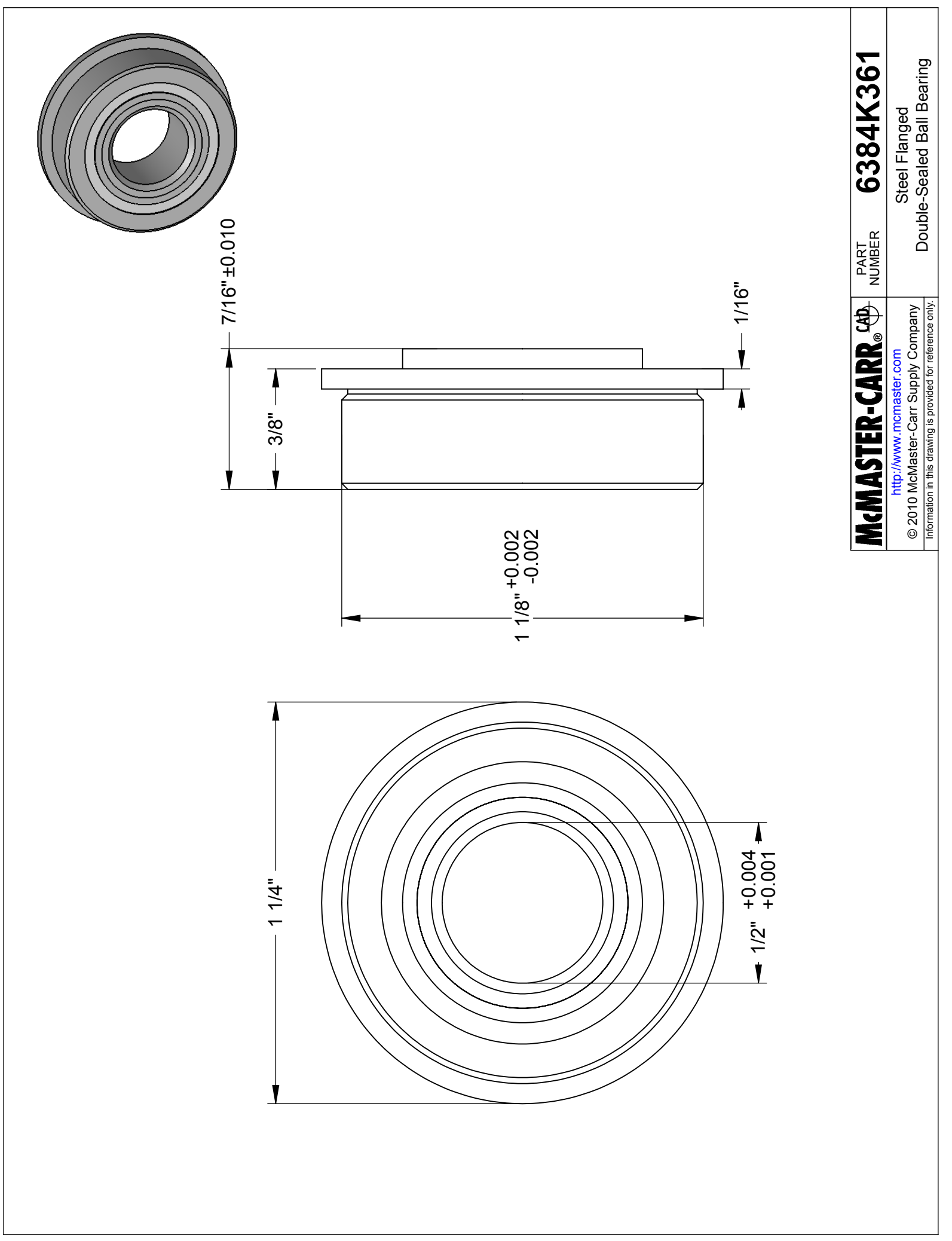




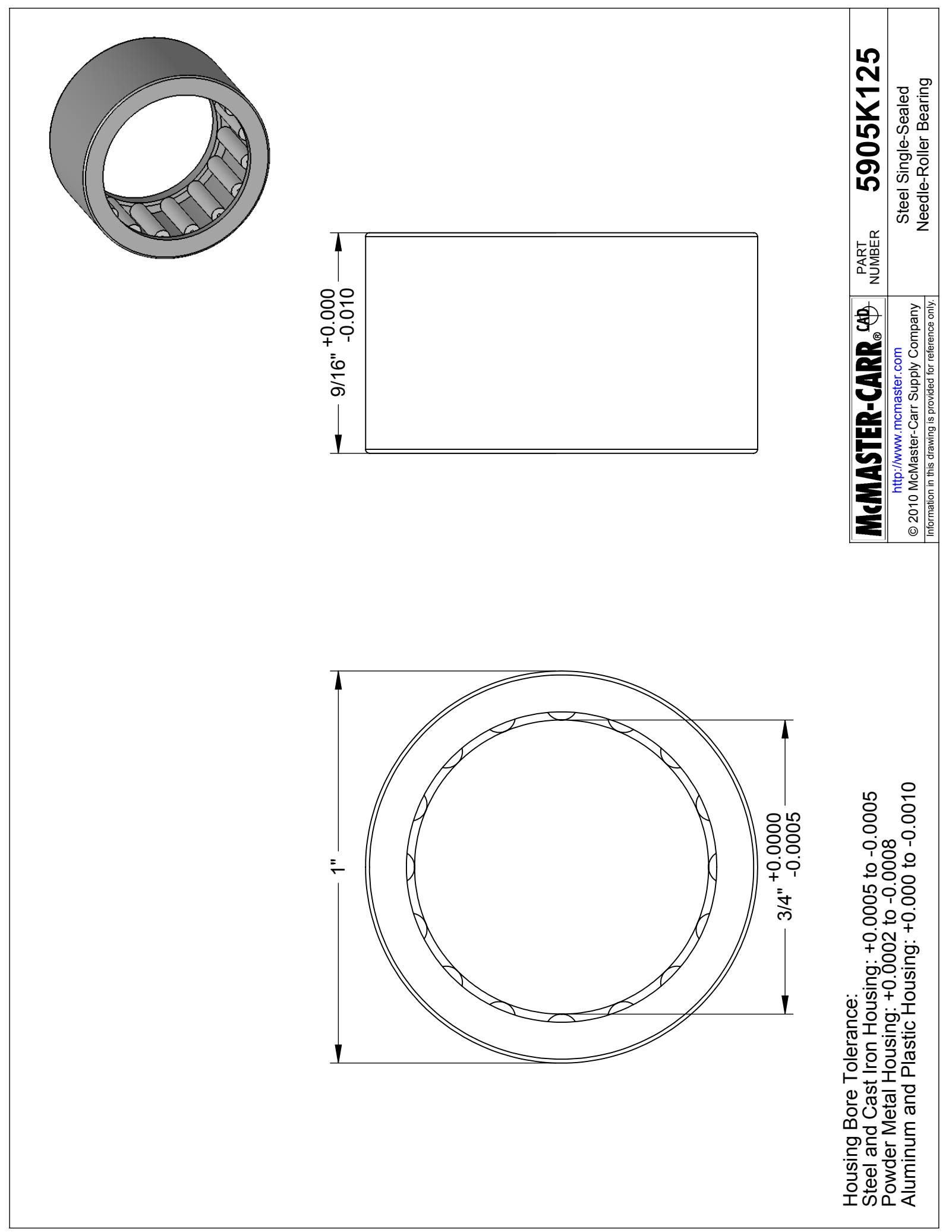




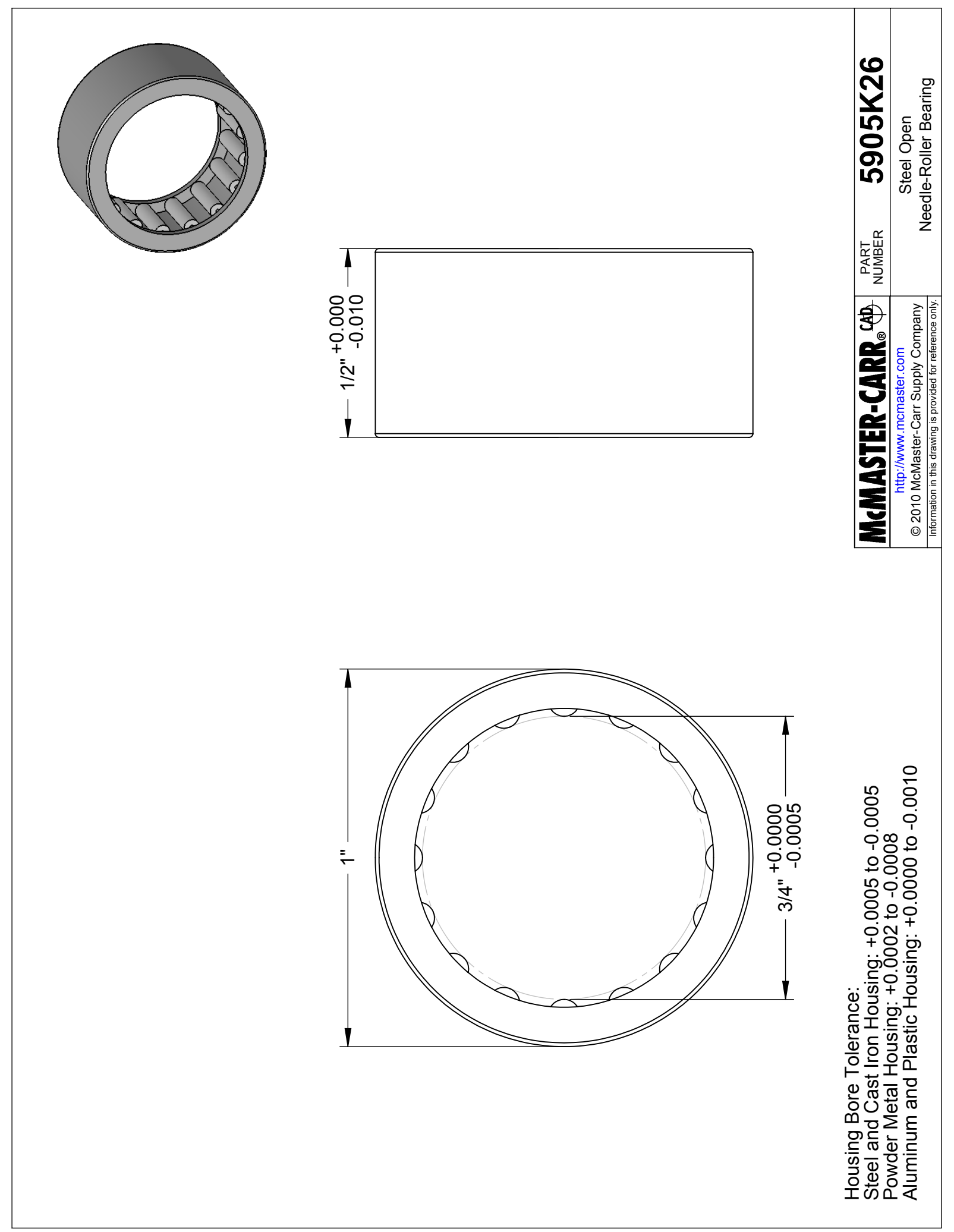




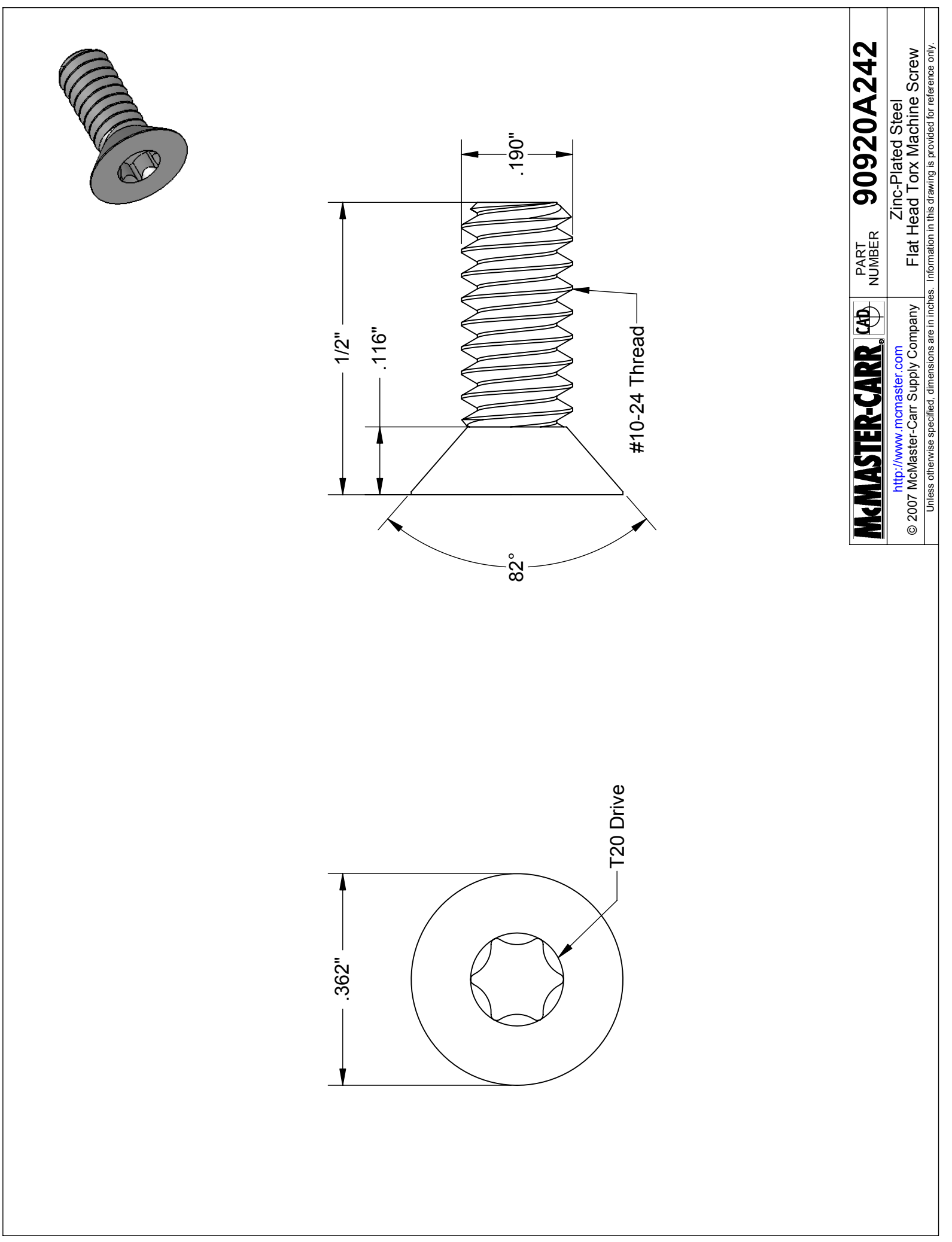




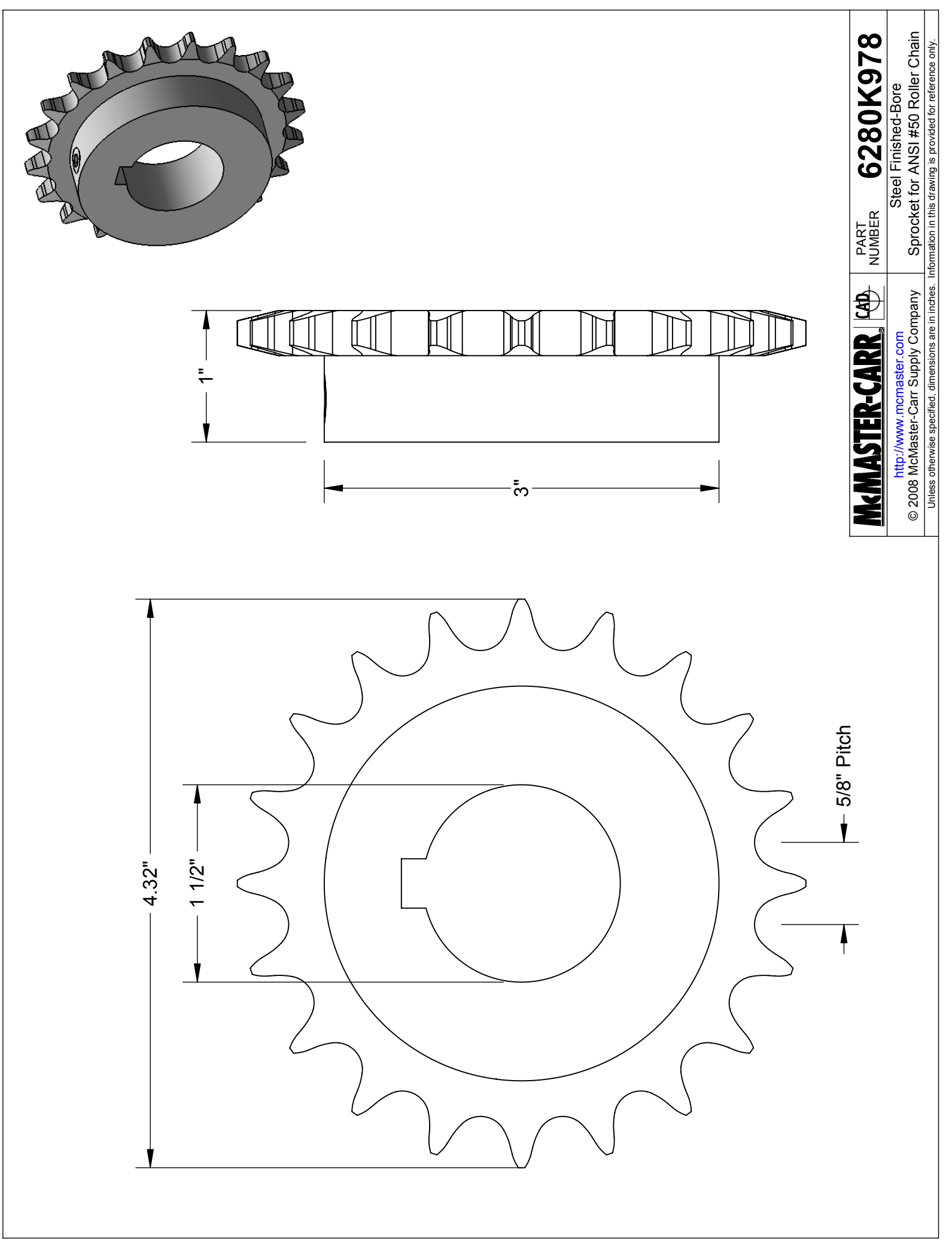

